\title{
ASSESSING QUALITY OF UPPER LIMB MOVEMENTS AFTER STROKE WITH PROSPECTS OF WEARABLE TECHNOLOGIES

$$
2\left(q_{1}, 9_{3}+9_{0} q_{2}\right)
$$$$
(4) R_{y}(\theta) R^{R}
$$

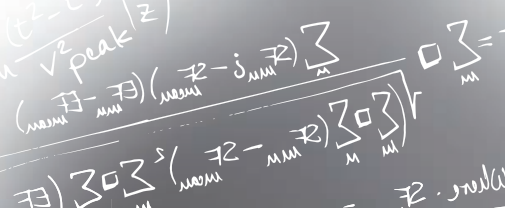

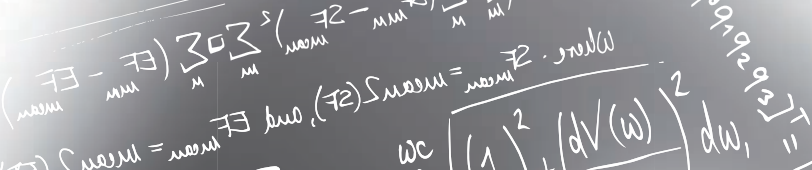

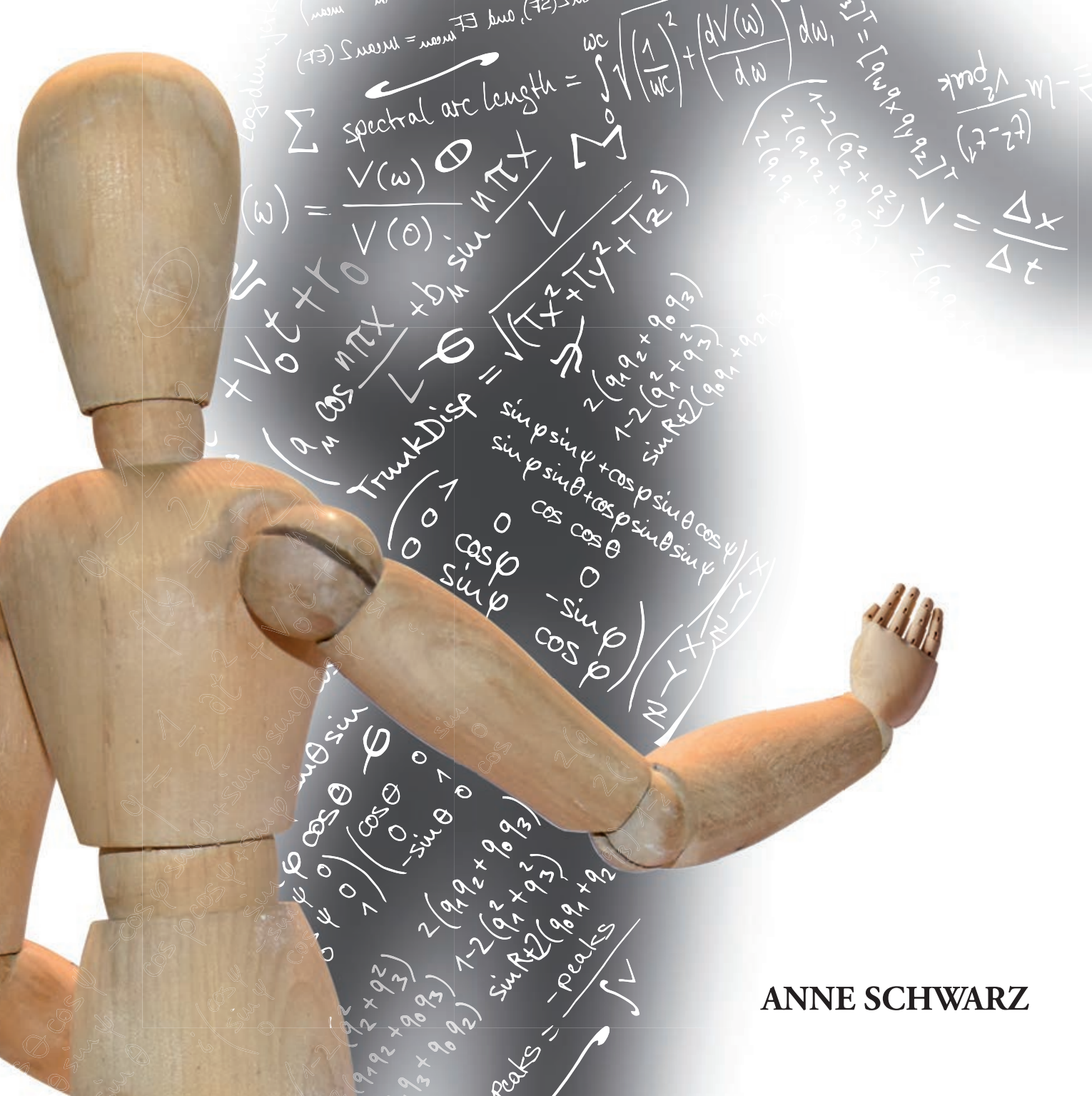





\section{Assessing quality of upper limb movements after stroke with prospects of wearable technologies}


Cover Stefan and Anne Schwarz

Layout Renate Siebes | Proefschrift.nu

Printed by ProefschriftMaken, Bilthoven

ISBN 978-90-365-5163-2

DOI $\quad 10.3990 / 1.9789036551632$

\section{이 2021 Anne Schwarz, the Netherlands}

All rights reserved. No parts of this thesis may be reproduced, stored in a retrieval system or transmitted in any form or by any means without permission of the author. Alle rechten voorbehouden. Niets uit deze uitgave mag worden vermenigvuldigd, in enige vorm of op enige wijze, zonder voorafgaande schriftelijke toestemming van de auteur. 


\section{Assessing quality of upper limb movements after stroke with prospects of wearable technologies}

\section{DISSERTATION}

to obtain

the degree of doctor at the University of Twente,

on the authority of the rector magnificus,

prof. dr. ir. A. Veldkamp,

on the account of the decision of the Doctorate Board,

to be publicly defended

on Wednesday the $21^{\text {st }}$ of April 2021 at 12:45

by

\section{Anne Schwarz}

born on the $10^{\text {th }}$ of May 1986

in Neubrandenburg, Germany 
This dissertation has been approved by:

Supervisors: $\quad$ Prof. dr. J.H. Buurke

Prof. dr. med. A.R. Luft

Co-supervisor: $\quad$ Prof. dr. ir. P.H. Veltink 


\section{Graduation committee}

Chairman/secretary: Prof. dr. J.N. Kok (University of Twente)

Supervisors: $\quad$ Prof. dr. J.H. Buurke $\quad$ (University of Twente, Roessingh

Research and Development)

Prof. dr. med. A.R. Luft (University of Zurich)

Co-supervisor: $\quad$ Prof. dr. ir. P.H. Veltink $\quad$ (University of Twente)

Members - internal: $\quad$ Dr. ir. B.J.F. van Beijnum (University of Twente)

Prof. dr. H. Rietman (University of Twente)

Members - external: Prof. dr. R. Gassert $\quad$ (ETH Zurich)

Prof. dr. K.S. Sunnerhagen (University of Gothenburg)

Dr. G. Verheyden (KU Leuven) 



\section{Contents}

Chapter 1 General introduction

Chapter 2 Systematic review on kinematic assessments of upper limb

movements after stroke

Chapter 3 Measures of interjoint coordination post-stroke across different upper limb movement tasks

Chapter 4 A functional analysis-based approach to quantify upper limb impairment level in chronic stroke patients: a pilot study

Chapter 5 Kinematic core-set of upper limb movements after stroke and their relationship across various upper limb activities of life

Chapter 6 Assessment of upper limb movement impairments after stroke using wearable inertial sensing

Chapter 7 General discussion

Summary

Samenvatting

Zusammenfassung

Acknowledgements / Dankwoord

About the author

Biography

Publications

Conference presentations 

General introduction 

It has been phrased by Tim Minchin (2013), that "science is not a body of knowledge, nor a belief system, it is just a term which describes humankinds' incremental acquisition of understanding through observation". To that, the level of observation should be selected according to the level of interest, where the targeted action is expected to happen. Observations of human movement, by either inspection or palpation, are one of the key elements in physiotherapy, when it comes to clinical reasoning about the cause and treatment approach to relieve disease threats. Nevertheless, these observational skills are mostly persondependent and based on the observers' expectations on what to search for and where to look at. Signal sensing can potentially overcome this subjective unilaterality.

The first observations of human movement go back to Aristotle (384-322 BC), who described movement speed to be linearly dependent on the exerted force of the person that performs the movement. Centuries later, Leonardo Da Vinci (1452-1519) was the first to study anatomy within the context of mechanics, followed by many others, such as Giovanni Alfonso Borelli (1608-1679), René Descartes (1596-1650), Galileo Galilei (1564-1642), Isaac Newton (16431727), and Leonard Euler (1707-1783) (van den Noort, 2011).

Nicolai Alexandrowitsch Bernstein (1896-1966) and his works, like "the co-ordination and regulation of movements" (1967), laid the cornerstones for modern human movement analysis. An increased number of technologies were developed in the years from there on, such as optoelectronic systems or surface electromyography that enabled accurate human movement analysis. Although, these technologies have shown to be extremely supportive in fields of research, such as sports or rehabilitation medicine, their applicability remains mostly far from applicability in usual care, even in high-income countries. Taking a glance on rehabilitative services after stroke, assessments of upper limb movement function and activities mainly rely on descriptions of movement behavior in therapist records, and time-based or observer-based scoring principles in standardized clinical scales. This level of observation results in relatively superficial and simplified information on movement quality that is unlikely to provide specific answers to the questions how movements evolve in physiological and pathological systems. The developments in wearable sensing technologies of the last decades have enabled widespread applications, by devices such as smartphones or activity trackers, offering continuous and accurate movement recordings based on accelerometry and other inertial sensing. The wearing comfort, appeal and functionality of these devices has guaranteed application fields in the personal life, research fields, and health care alike (Haghi et al., 2017). Nevertheless, the acquisition of such movement data results in large packages of raw data, that needs to be further processed to become informative and 
interpretable. The challenge on selecting reliable, sensitive, and relevant measures highly depends on such postprocessing steps, ranging from filtering technics, equations, decision trees, and calculation steps that itself require human observations and interpretations.

This thesis aims to investigate quality of upper limb movements after stroke by use of kinematic measurements to improve assessment strategies based on technological opportunities and clinical knowledge on pathophysiology and observations in daily practice. This general introduction is separated into the chapters of "stroke epidemiology, diagnostics and classifications", followed by "upper limb sensorimotor control”, "upper limb impairments after stroke and their course of recovery", "upper limb motor assessments after stroke", and "prospects of wearable technologies". After a brief summary on the "challenges within the SoftPro project", the main research questions of the thesis were laid down.

\subsection{Stroke epidemiology, diagnostics and classification}

Although the number of strokes has been reduced by $21 \%$ globally, it still stands for the second largest cause of death after ischemic heart disease (Johnson et al., 2019). According to the Global Burden of Disease (GBD) 2016 Lifetime Risk of Stroke Collaborators, stroke was the second largest cause for disability in adults represented by more than 80 million stroke survivors worldwide in 2016 (Johnson et al., 2019). Based on the facts of population growth and the increased lifetime expectations, the number of strokes is expected to increase likewise. It has been suggested that until 2050 the number of strokes will be doubled, especially in the persons above 75 years of age (Gorelick, 2019). Because of the professionalized acute stroke care management, especially in the high-income countries, and improvement in therapeutic interventions such as thrombolysis or thrombectomy, an increase in persons with chronic disabilities due to stroke is to be expected (Alawieh et al., 2018). In 2017, 1.12 million stroke incidences were registered in the European Union, with 9.53 million survivors, 0.45 million deaths, and predictions of a 27\% increase of the number of strokes by 2047 (Wafa et al., 2020). Likewise, an increase healthcare costs based on summed up direct and indirect costs of 20 billion and 25 billion in 2015 of needs to be considered in the European Union (Stevens et al., 2017; OECD, 2016).

A stroke is defined as a central nervous system infarction based on objective evidence of cerebral, spinal cord or retinal focal ischemic injury in a defined vascular distribution or clinical evidence of the mentioned injuries based on symptoms persisting over 24 hours 
or until death, including cerebral, intracerebral, subarachnoid hemorrhage, and cerebral venous thrombosis (Sacco et al., 2013). Ischemic strokes account for $85 \%$ of all strokes, while hemorrhagic strokes were less frequent but tend to have more pronounced neurological impairments and higher mortality rates (Schepers et al., 2008). Though the differentiation between ischemic and hemorrhagic stroke with diagnostical tools is critical in the hyperacute and acute phase, impairment-based functional differences between both stroke types tend to vanish in the chronic stage at around three to six months post-stroke (Schepers et al., 2008).

The initial deficits can include weakness of one side of the face, one arm and/or leg, slurred speech, and/or perceptive function (Weimar et al., 2002). The severity of clinical symptoms in the acute stage is frequently evaluated by the Oxfordshire or Bamford's classification (Bamford, 2000) and the National Institute for Health Stroke Scale (NIHSS). The Bamford classification differentiates four syndromes; the total anterior circulations syndrome (TACS) including motor deficits, higher cerebral dysfunctions and homonymous hemianopia, the partial anterior circulation syndrome (PACS) including two of the above-named symptoms, the lacunar syndrome (LACS) with pure motor or sensory deficits and posterior circulation syndromes (POCS) including cerebellar ataxia and brain stem signs, such as dizziness and nausea. The NIHSS assesses the severity of stroke by clinical examination of eleven items, reflecting alertness, visual function, motor function, speech function, sensation, and perception, on a scale ranging from 0 to 42 (Brott et al., 1989). It has been shown, that the NIHSS strongly predicts the stroke outcome (Adams et al., 1999), by discriminating no $($ NIHSS $=0$ ), minor (NIHSS $=1-4)$, moderate (NIHSS = 5-15), moderate to severe (NIHSS $=16-20)$ and severe symptoms (NIHSS $=21-42$ ). Furthermore, evidence on the relationship between stroke tissue loss and the clinical outcome suggests that larger tissue loss corresponds to more severe and multimodal deficits (Alexander et al., 2010).

The impairments and long-term consequences after stroke are highly variable given the complexity of cerebral functions and interlaced networks, as illustrated for the main sensorimotor central nervous pathways in Figure 1.1. Stroke-related deficits can span from impaired movement function, like complete paralysis of the contralesional upper limb to less apparent dysfunctions, such as sensory deficits or higher cognitive dysfunctions. Recently, observational studies found that impairments of upper limb motor function contribute most to limitations in participation in terms of leisure and social activities, besides independence in daily life, balance function, and sensory function (Ahn et al., 2018; Carey et al., 2018). 


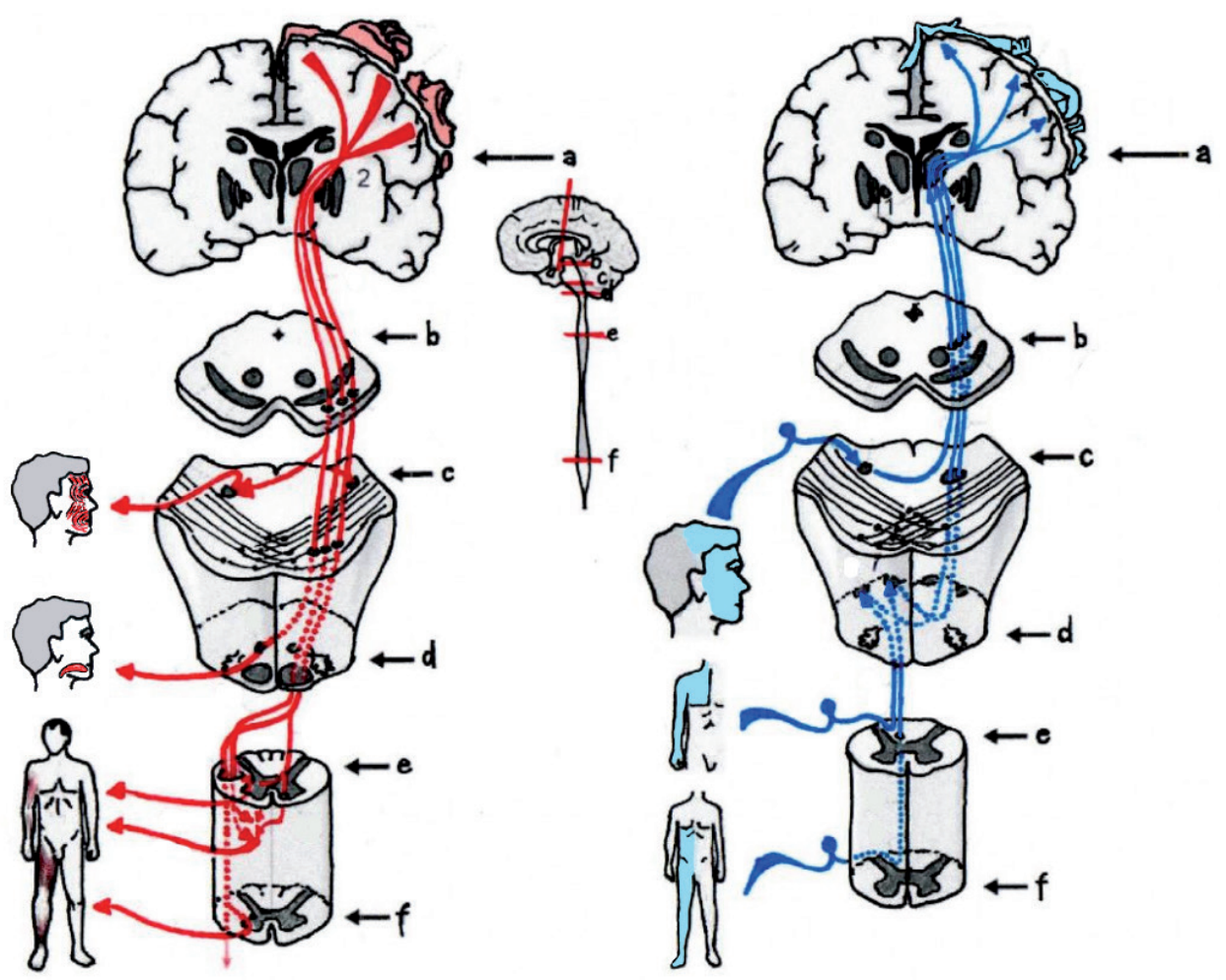

Figure 1.1. Corticospinal tract in red (left) and medial lemniscus pathway in blue (right) (adapted from Netter).

\subsection{Upper limb sensorimotor control}

The human upper limb consists of the shoulder girdle, the glenohumeral joint, radioulnar joint, the wrist and the five digits, resulting in large number of degrees of freedom that can be coordinated in tremendous different ways (Santello and Lang, 2015). Three degrees of freedom can be differentiated in the glenohumeral joint with range of motions from $20-40^{\circ}$ in extension and adduction to $150-180^{\circ}$ in flexion and abduction, and $50-90^{\circ}$ in internal and external rotation. Elbow joint motions are represented by one degree of freedom ranging from $-10^{\circ}$ or $0^{\circ}$ of elbow extension to around $135^{\circ}$ of elbow flexion. Forearm pronation and supination accounts for another DOF with $80-90^{\circ}$ in each direction. The wrists consist of flexion and extension motions around $40-70^{\circ}$ and abduction and adduction of around 20$40^{\circ}$ (Lea et al., 1995). Fifty-four muscles groups can be differentiated per arm allowing the performance of widespread actions and functions. The functions of the human upper limb span from communication and gesturing, stereognosis and sensing of the environment, from 
gross to fine manipulation of various objects, providing balance and support over to highly specified dexterous movements of the five digits. Upper limb movements are variable and specifically shaped by the task and environment within which they are performed (Shumway Woollacott, 2017). Likewise, requirements for upper limb activities range from increased strength but little dexterity demands, such as sweeping or hammering, to those requiring selective grasp and dexterity, such as screwing a small bolt into hardware or to thread a needle.

It is not surprising that the cerebral representation of the hand and arm has shown to be vast and complicated, as explored in imaging studies, spanning from sensorimotor integration on the level of the brainstem and thalamus, the limbic system to the dorsolateral prefrontal cortex and parietal cortex (Nudo et al., 2006). Motor control to generate upper limb actions, relies on a complex network of higher cortical structures, such as the primary motor cortex, subcortical motor-regulating centers, such as the basal ganglia and the brainstem, the cerebellum, the first and second motor neurons in the spinal cord and musculoskeletal end-effector organs, as shown in Figure 1.2. Functionally the dorsolateral cerebral system is responsible for regulating selective goal-oriented movements of the hand, while postural control and balance

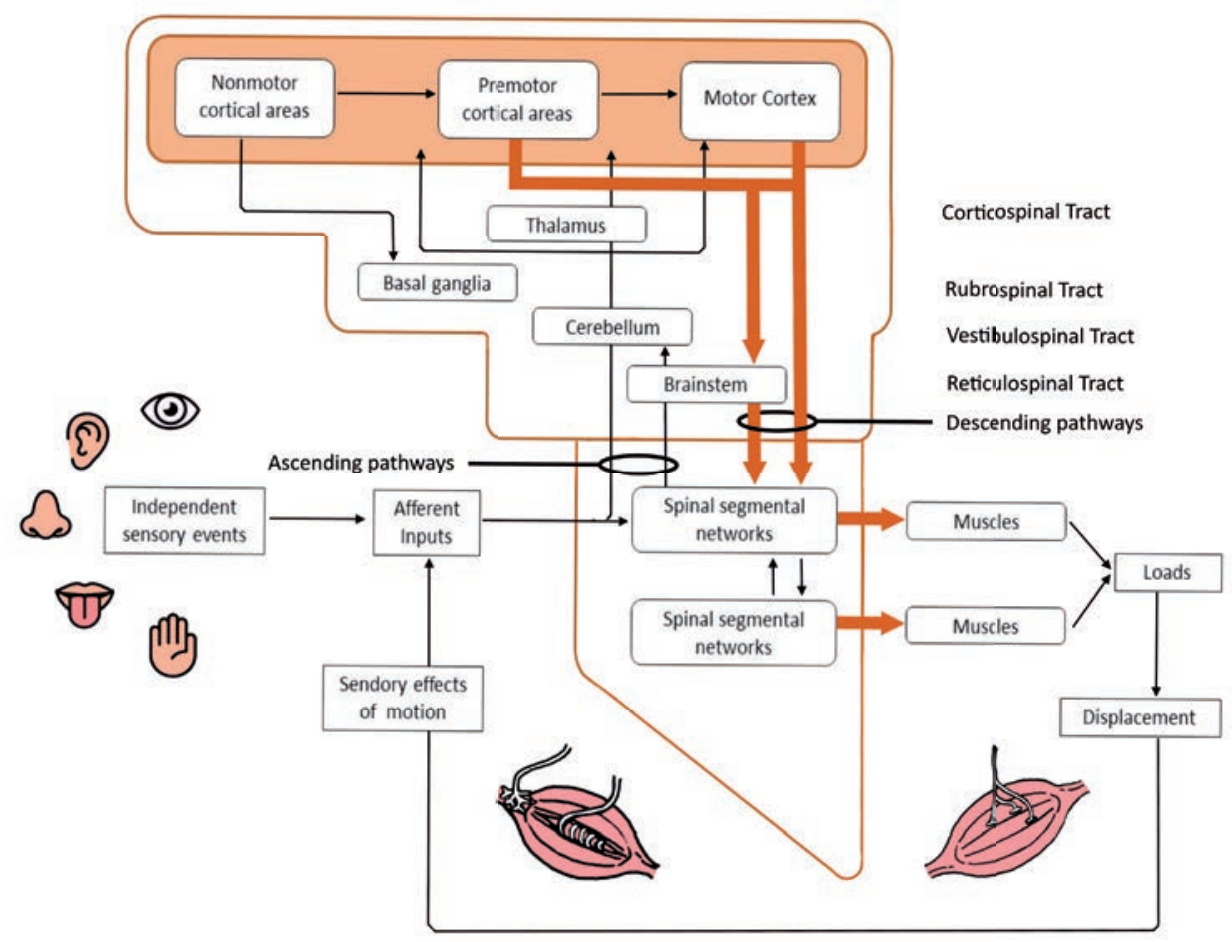

Figure 1.2. Sensorimotor network from cortical level to end effector (adapted from Shumway-Cook and Woollacott, 2017). 
is regulated through the ventromedial system (Kandel et al., 2000). Upper limb activities, especially reach-to-grasp movements that require physical interaction with the environment rely on feedforward processes including visual information to localize the target and target characteristic, such as weight prediction (Lukos et al., 2007), before the motor program is selected (Santello et al., 2002). For example, when one attempts to grasp a cold wet milk bottle off the fridge feedforward processing results in expectation of cold sensory information and increased force of grasp to prevent the bottle from slipping through the fingers.

Despite these findings, the question of how human upper limb movement control and coordination is organized, processed and recovered from injury has been addressed and includes the concept of synergies ever since Nicolai Bernstein (Bernstein, 1947) and underwent ongoing contrasting discussions (Santello and Lang, 2015). Synergies are defined as a collection of relatively large numbers of degrees of freedom that behave as a single functional unit. Different combinations of joints and muscles are used, while retaining the stability for the whole movement (Bernstein, 1967).

It remains unknown on which level the central nervous system selects the optimal set of DOFs to carry out a task, whether the CNS controls through activation of individual motor units or simply cares about task accomplishment (Latash et al., 2007; Tresch and Arc, 2009). Likewise, in the pathological case of stroke-related changes in the sensorimotor central nervous system, the answer to the questions on how recovery of motor function evolves, what it is dependent on, and which interventional strategies exist to achieve the maximum possible functional and structural restoration, keeps concerning the fields of neuroscience and rehabilitation research.

\subsection{Upper limb impairments after stroke and their course of recovery}

Disruptions in the previously described upper limb sensorimotor control loop, as due to stroke, can lead to small-scale subliminal changes or large-scale and complex impairments, disabilities, and barriers in daily life of the person and their relatives. Frequencies of upper limb dysfunctions have been reported in $46-85 \%$ of stroke survivors in the acute stage (Jorgenson, 1999; Persson et al., 2015) and have shown to recover in about $60 \%$ of those stroke subjects with some voluntary function in the fingers and shoulder within the first 72 hours after stroke (Nijland et al., 2010). Those subjects with no voluntary function in the fingers and the shoulder are at risk of experiencing vast limitations in activities of 
daily life and long-term side effects, such as spasticity, pain, and contractures (Allison et al., 2015). Limitations of upper limb function are frequently explored by stroke survivors, often resistant to change and challenging to be framed within the above-mentioned central nervous sensorimotor control loop.

The main upper limb impairments due to stroke have been summarized as paralysis, paresis or weakness, loss of independent joint control and a loss of dexterity that might lead to non-use or bad use (Raghavan, 2015). Unwanted movement synergies reflecting the loss of independent joint control have been described as a common phenomenon related to stroke since the earliest documentations of stroke (Twitchell, 1951; Brunnstrom, 1970). The synergistic and stereotypical movement patterns, such as the flexor synergies in the upper limb movements of stroke subjects are causal for a limited range of motion in the affected joints and an overall limited workspace (Dewald et al., 1995). The underlying mechanisms of pathological synergies have been examined in terms of cortical control (McMorland et al., 2015, Roh et al., 2013), the descending pathways and muscle activation (Yao et al., 2009), as well as in relation to sensory loss (McCrea et al., 2005). Nevertheless, control mechanisms and centers of synergistic movement patterns remain unknown, as well as the delineation between the pathological and physiological case. Movement synergies are usually used to characterize patterns of upper limb motor control. The term synergy describes the stereotypical coupling of two or more segments or muscles into building blocks for generating coordinated upper limb movements (McMorland et al., 2015; Roh et al., 2013), that could tag physiological and pathological processes alike.

Based on pioneering works by Thomas E. Twitchell and Signe Brunnstrom, a relatively definable course of recovery was described, from "initially nearly flaccid hemi paralysis" to a certain degree of selective motor control through the course of stroke recovery (FuglMeyer et al., 1971). A series of special events regarding the recovery process of stroke were observed and described (Brunnstrom, 1960). Although reasonable variation was found in the recovery process, the restoration of motor function in the hemiplegia patients followed a general pattern in which certain phenomena were remarkable during distinct stages or phases of the process. After the immediate onset of hemiplegia, where the affected limb is completely flaccid and felt heavy when moved passively with little or no muscular resistance to movement, a phase characterized by increased activation with the emergence of hypertonicity or spasticity and synergistic movement patterns develops. Any attempt of voluntary movement resulted in some of the components of the limb synergies, e.g., components of the flexor or extensor synergy of the upper arm, as illustrated in Figure 1.3. 
(A)

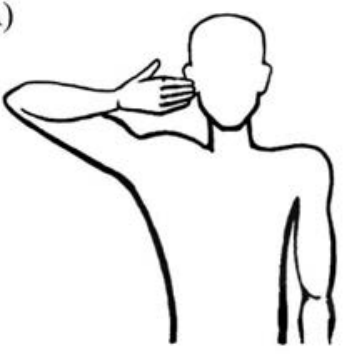

(B)

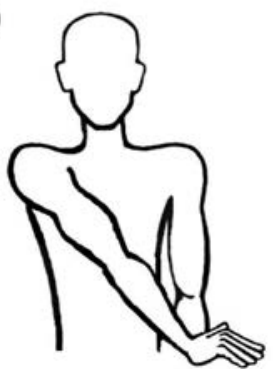

(C)

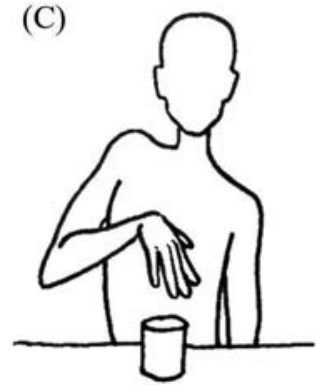

Figure 1.3. (A) The flexor synergy was defined in coupled elbow flexion with shoulder abduction and flexion, and forearm supination. (B) The extensor synergy defined by coactivation of elbow extension and shoulder adduction and forearm pronation. (C) Abnormal movement behavior in functional task of reach to grasp.

Further the recovery stage divides in those subjects, where spasticity tends to decrease with an increase of voluntary movement control in out-of-synergy movements and those subjects, where voluntary function is absent or reduced and abnormal tightness and stiffness worsens. This prescribed course of recovery has not been reproduced in later study and the trend of recovery might by differently perceived in nowadays clinical practice. The course of recovery has later been profiled using clinical assessment scales and neurophysiological measures such as transcranial magnetic stimulation to test the integrity of the corticospinal tract (Stinear et al., 2007), confirming a logarithmic trend of functional improvement within the first three months that is largely determined by the fact, if initial voluntary activitation of shoulder abduction and finger extension are producible.

The symptoms of UMN damage require differentiation from damage to lower motor neurons which would manifest with weakness, muscle atrophy, hypotonia, hyporeflexia, fasciculations, and fibrillation (Mayer et al., 2003). The symptoms of UMN syndrome can separate into negative and positive symptoms. Negative symptoms include weakness, decreased motor control, and easy fatigability. A unique characteristic of UMN syndrome is its tendency to affect specific muscle groups. The weakness caused by UMN syndrome will predominantly affect the extensors of the arm and flexors of the leg. Positive symptoms include increased muscle activity, such as spasticity, clonus, hyperreflexia, synkinesias and co-contractions (Emos et al., 2020).

Improving the understanding of the mechanisms contributing to movement restitution and the establishment of abnormal movement behavior relies predominantly on specific and sensitive measures and assessment tool that enable the detection of differences and changes. 


\subsection{State of the art in upper limb assessments after stroke}

Measurements of upper limb movements after stroke have been performed by use of a variety of clinical scales ranging from observer-based clinical scales, instrumented tests to patient questionnaires, covering different aspects of disabilities and functions according to the International Classification of Functioning and Health, the ICF (WHO, 2001), as illustrated in Figure 1.4. Different outcome measures for assessing the upper limb after stroke can be identified in clinical practice and research, while barriers in comparability and standardization were described. Systematic reviews identified around 53 different upper limb assessments, of which 13 met the criteria set for psychometric properties (Alt-Murphy et al., 2015). The Fugl-Meyer Assessment of the Upper Extremity (FMA-UE) has been shown to have the highest level of measurement quality and clinical utility (Alt-Murphy et al., 2015) and was the most commonly reported outcome measures with 37\% (Santisteban et al., 2016). Nevertheless, the FMA-UE does not assess functional arm and hand movements. The challenge of selecting the right outcome measure to assess upper limb functions after stroke is apparent in light of the various available measures and the question, on which level the characteristics and their behavior are thought to be observed. As a matter of course, there is no single measure that is specific to all aspects of upper limb function and activities, recovery and outcome after stroke (Persson, 2015).

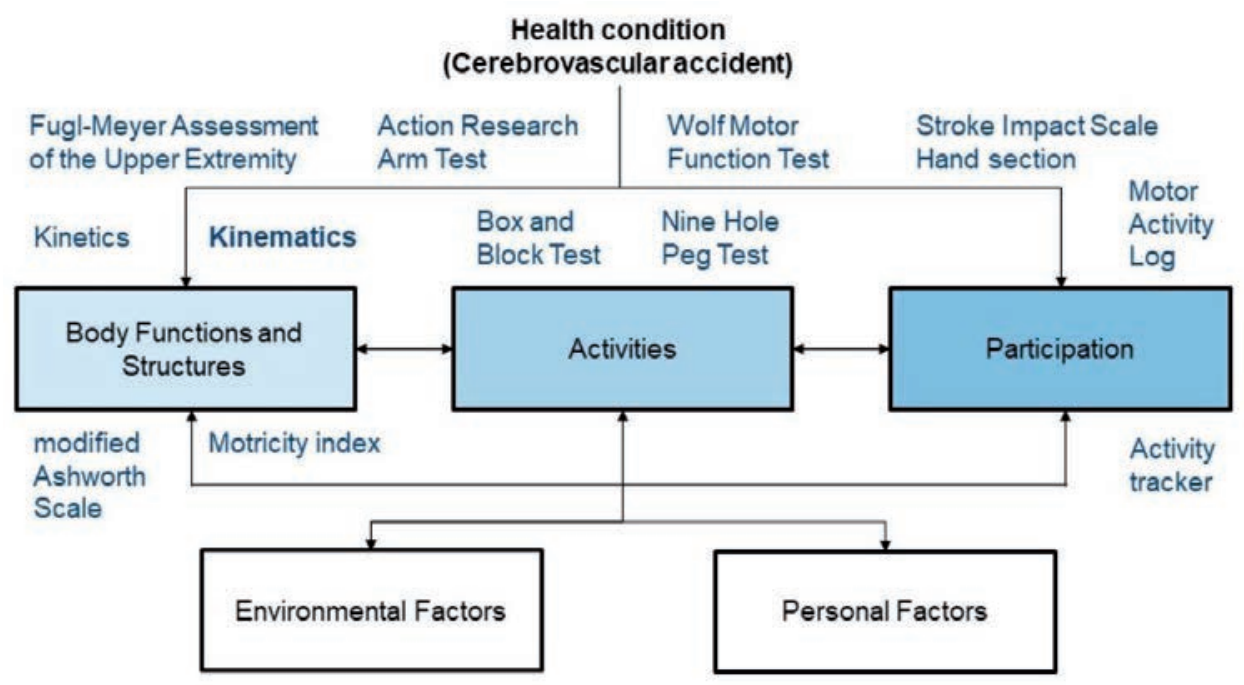

Figure 1.4. ICF Framework (adapted from WHO, 2001). 


\subsubsection{Body function and structure level}

The upper motor neuron syndrome has been defined as one of the main consequences of stroke and describes the combination of positive and negative symptoms, such as weakness and hypertonicity and co-activation. Muscle strength (ICF b730) can be measured with the medical research council or the motricity index. Aspects of muscle tone, such as increased resistance against passive movements (ICF b735), is usually assessed with the modified Ashworth Scale. In contrast to musculoskeletal diseases, specific muscle or joint functions, such as active and/or passive range of motion (ICF b710) were less often reported in subjects after stroke, as strength and range of motion after stroke were thought to be dependent on synergies (Fugl-Meyer et al., 1975). Quality of upper limb movements can be described as voluntary and selective movement control that are classified under the ICF body function level domain (b760). The FMA-UE provides information about the coordination of specific voluntary joint control on a score range 0 to 66 divided into 18 items for arm, 5 items for wrist and 7 items for hand movements as well as 3 for coordination and speed. The items follow a hierarchical structure from movements within synergies (e.g., combined elbow flexion and shoulder flexion), combined synergies (e.g., combination of elbow extension and shoulder flexion) and out of synergies (e.g., combination of elbow extension and shoulder abduction) as well as an order from proximal to distal movements. The FMA-UE takes 6-30 minutes and has attested a high test-retest and interrater reliability and construct validity in subacute and chronic stroke patients with good clinical utility (Gladstone et al., 2002; Alt-Murphy et al., 2015).

\subsubsection{Activities and participation level}

On the level of activities and participation the upper limb can be framed in lifting and carrying objects (ICF d430), fine hand use (ICF d440), and hand and arm use (ICF d445). Hand and arm use relies on displacing and manipulating activities, such as pulling or pushing objects, reaching, throwing, or catching and consist of complex coordinated multijoint movements, required to move objects or to manipulate them by using hands and arms, such as when turning door handles or turning or twisting the hands or arms. Fine hand use includes coordinated actions of handling objects, such as picking up, grasping, manipulating, and releasing.

Clinical scales, such as the Action Research Arm Test (ARAT), the Wolf Motor Function Test (WMFT), the Box and Block test (BBT) and the Nine Hole Peg Test (NHPT) are used to assess grasping and displacement activities of different object sizes by means of movement 
time quantities and in the case of the ARAT and WMFT additional movement quality rating on an ordinal scale.

Furthermore, semi-structured interviews and questionnaires exist to evaluate the subjects' self-perceived functionality in activities of daily life, such as the ABILHAND, the SIS hand section and the Motor Activity Log (MAL). The ABILHAND consists of 23 bimanual activities that were scored on a 3-point ordinal difficulty scale. The MAL-14 reflects reallife functional performance, based on 14 arm activities that were rated according to their amount of usage and quality of usage when performed with the affected upper limb.

\subsection{Prospects of wearable technologies}

In contrast to clinical examinations and scales, technologies offer kinematic measurements to capture and analyze movement behavior objectively and comprehensively and thereby sensitively discriminating physiological from pathological movement behavior and functional restitution from compensation along the course of recovery after stroke (Kwakkel et al., 2017).

Since the 1970s, when the first video analysis was applied to record human movement parameters, wearable technology to measure motion has been increasingly developed and spans from smart phones or mobiles containing accelerometers, wireless, textiles and garments to accelerometer or inertial measurement units (IMUs). Flexible angular sensor and E-textiles, such as stretch sensing fabric or electrical leads, provide textile solutions to kinematic measurement systems. Wearable sensor-based systems and miniaturization of devices have triggered rehabilitation technologies by offering advantages, such as low cost, flexible application, remote monitoring, and comfort, while allowing independent training and the provision of feedback (Patel et al., 2012; Wang et al., 2017). A review on interactive wearable systems for upper body rehabilitation identified 45 publications with $84 \%$ of the studies on accelerometer and inertial measurement units (IMU) that were mostly placed at the trunk, upper arm, forearm, wrist and finger (Wang et al., 2017). Inertial measurement units are small, low-powered electromechanical sensors that potentially enable dynamic and flexible three-dimensional human motion analysis (Cuesta-Vargas et al., 2010). Usually consisting of a gyroscope to capture 3D angular velocity and an accelerometer that measures linear acceleration in $3 \mathrm{D}$, the fusion of accelerometer and gyroscope signals allow the estimation of orientation and position. In the static position, the gyroscope provides information of the orientation and the accelerometer measures gravitational acceleration. 
Both signals are exposed to risk of drift over time due to integration and need to be carefully handled by applying sensor-fusion algorithms and filters, such as the Kalman filter (Paulich et al., 2017). Frequently, magnetometers are included as well to provide estimations of the orientation in relation the magnetic north.
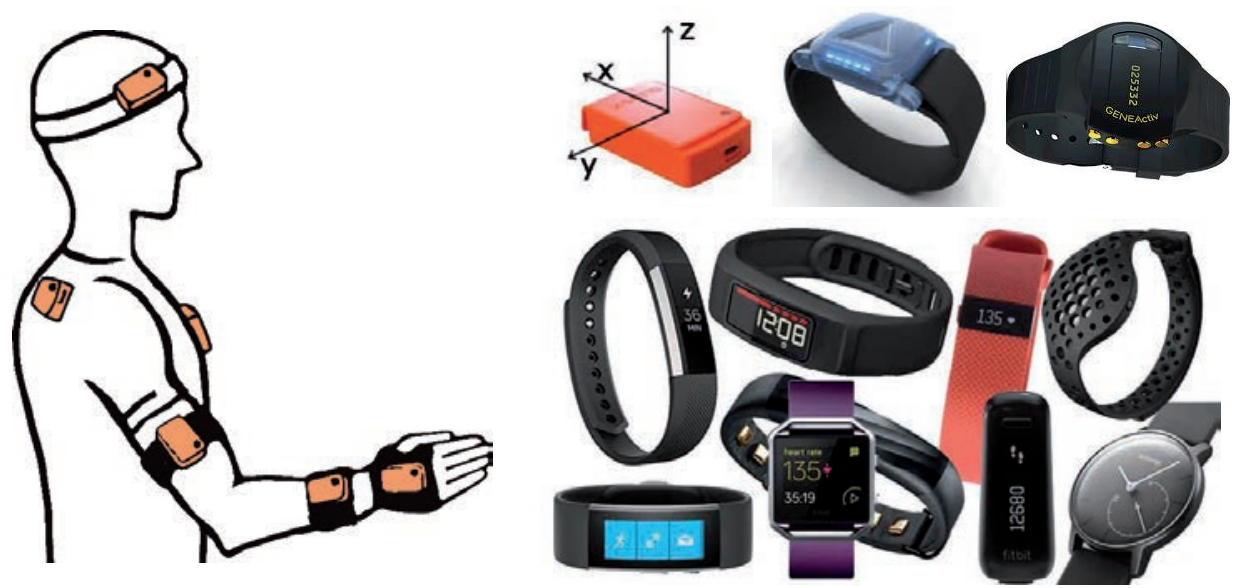

Figure 1.5. Upper-body IMU-based sensing system (left) and activity trackers (right).

Two IMUs can be used to calculate the joint angles of the connecting joint (Müller et al., 2016). IMU applications range from wrist-worn single units that provide measures, such as activity counts, to more extensive systems such as the Xsens system consisting of 17 IMUs that allow full body motion analysis of the main 23 human body segments and the 22 joints.

Based on previous investigations of the Interaction System, metrics for upper and lower extremity function, and posture and activity detection algorithms were developed (Klaassen et al., 2015; van Meulen et al., 2015) that have shown to be applicable in non-structured daily life measurements of subjects after stroke (Held et al., 2018). Upper limb metrics, such as reaching counts and $2 \mathrm{D}$ workspace area, have shown to provide additional information to clinical assessments, when recorded during supervised non-structured activities of daily living (Held et al., 2018).

Besides these promising trends in wearable motion tracking, qualitative measures and applied metrics are variable and largely depending on the processing-steps. The data has to be managed and processed to derive meaningful information (Patel et al., 2012). Wang and colleagues identified three groups of outcome measures including range of motion, amount of use and body segment postures. Systems, like the Xsens MVN Awinda system, 
that combine a set of sensors into a biomechanical human model enable comprehensive data acquisition of the individual segment positions and accelerations as well as joint angles. Nevertheless, the system has not yet been applied to capture and analyze upper-limb motions and its subcomponents qualitatively in different semi-structured upper limb daily life activities. The possibility to detect relevant aspects of upper limb movement quality by wearable technology would not only be useful for implementing objective assessments of movement quality in clinical practice but also for developing training solutions to extend the amount of training beyond the personal therapy sessions, in the long-term.

\subsection{Challenges within the European Project SoftPro}

All research performed and described in this thesis was funded through the European Union's Horizon 2020 research and innovation

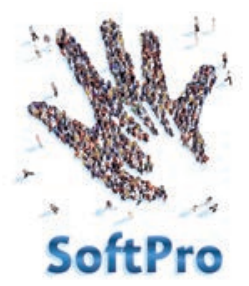
programme under grant agreement No. 688857 (SoftPro - Synergy-based open-source Foundations and Technologies for Prosthetics and Rehabilitation) that started in March 2016 and finished in March 2020.

Twelve partners from different fields, such as engineering, technical, neuroscientific, industrial, and clinical partner institutions collaborated in the project to provide humaninspired robotic technology solutions to support upper limb amputees and upper limb rehabilitation after stroke. The main pillars to achieve the project goals consisted of basic research on synergy-based arm and hand control, interfaces from natural to artificial, multimodal force or kinematic sensing, biomechanics and control of human-machine interaction, assessment methodology and technology, the development and refinements of tools for prosthetic users and upper limb support and rehabilitation, supra-numerical limbs, and clinical and user-based feedback, as indicated by the frequent feedback loops in Figure 1.6. Figure 1.6 illustrates the structure of these pillars or work packages and their interrelation.

The project goals related to this thesis were first, to improve the understanding of post-stroke upper limb motor control under natural movement execution with a focus on synergybased coordination aspects, and secondly, to develop sensitive assessments of upper limb movement control by use of wearable and easy-to-use technologies. The characterization of synergy-based movement control in physiological and pathological conditions, such as stroke (in WP1, Figure 1.6), is intended to support the development of sensitive assessment tools (in WP5, Figure 1.6) and new opportunities for natural, less-consumable device control. 
Synergistic movements can be defined and measured in different ways, on the level of motor units, muscles and/or joints and research debates about the regulatory centres are ongoing. Nevertheless, a common sense exists that synergies reflect multi-joint coordination within a lower dimensional space than the number of dimensions involved. Synergistic patterns of voluntary muscle activity and multi-joint coordination are supposed to allow dimensionality reduction and flexibility (Bernstein, 1967).

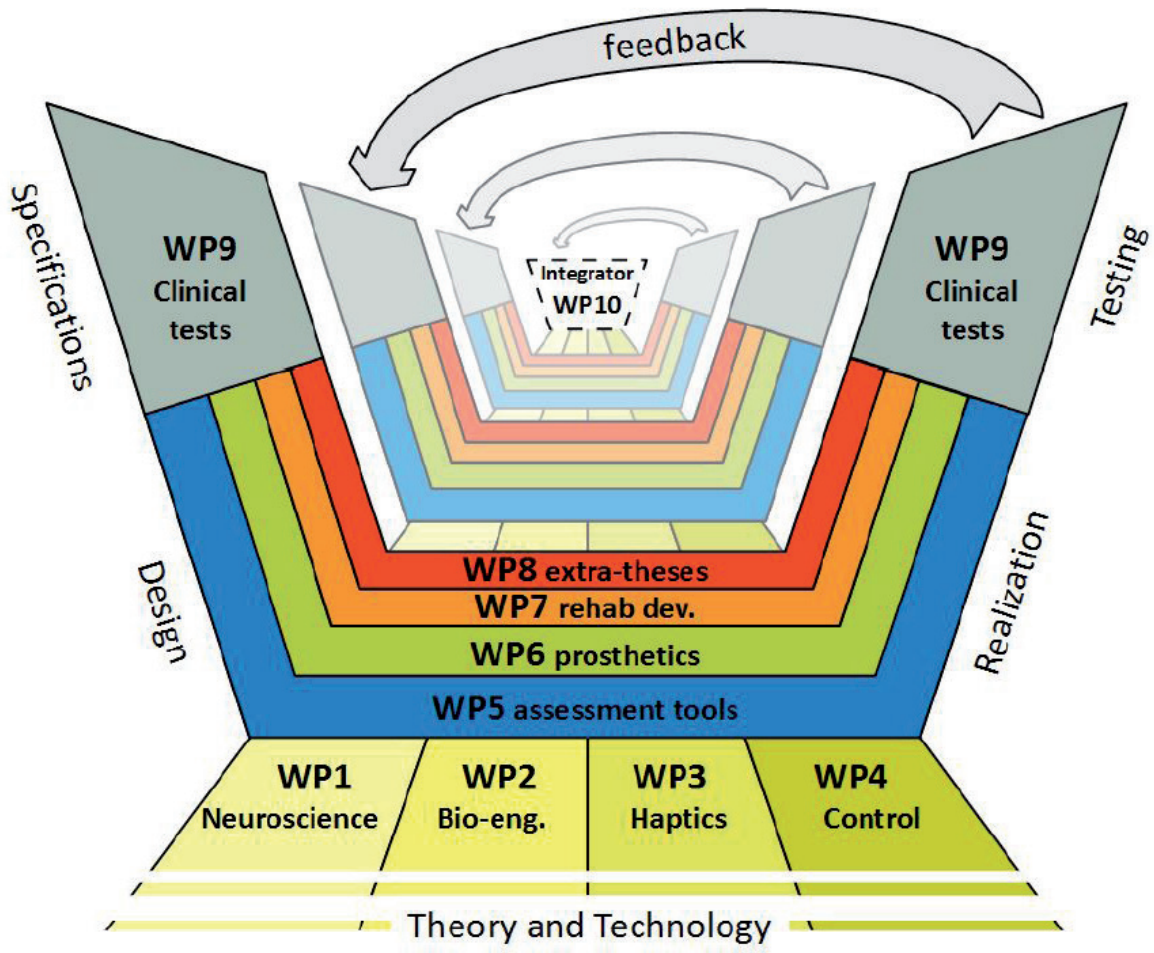

Figure 1.6. Iterations of the work packages in the Y-shape, toward the final project integrator.

This thesis contributes to the SoftPro-project goals of improving our understanding in synergistic movement control. To ensure the studying of natural human movement behaviour, innovations in wearable unobtrusive biological signal sensing system for monitoring and assessing execution of ADLs were targeted within the project. In WP1, the partners collected a multimodal, multicentre dataset on a shared set of activities of daily life, ranging from inertial sensors, surface EMG, and EEG caps and functional MRI to exhaustively characterize upper limb movement synergies in the physiological and pathological case. 


\subsection{Research questions}

The main goal of this thesis is to investigate the prospects of wearable technology to assess aspects of upper limb movement quality in subjects after stroke. Assessing upper limb multijoint coordination in the damaged nervous system remains a challenge due to the complex and unknown mechanisms that interplay in the physiological and pathological system and the large amount of variability in upper limb usage. Additionally, the complexity and variability of upper limb function presents a barrier in the establishment of comparable assessment standards. Monitoring upper limb function after stroke is of importance for selecting effective rehabilitation approaches and requires precise analysis within the context of the subjects' pre-stroke upper limb functionality in daily life. Within this thesis it is aimed to determine useful kinematic parameters and assessment set-ups to evaluate upper limb function in the most accurate, ecologically valid, and natural manner. The five chapters of this thesis are based on three studies, one systematic literature review, one cross-sectional observational study and one pilot study to address the following research questions:

- What is the state of the art in upper limb kinematic assessments in stroke survivors, including the assessment protocols and outcome parameters selected?

This question was addressed in an overview of state of knowledge in literature regarding upper limb kinematic assessments in stroke survivors by performing a systematic review that has been prospectively registered on prospero (CRD42017064279). Chapter 2 provides an overview of the measurement systems, the assessment movement tasks and metrics identified for upper limb kinematic assessments after stroke. Subsequently, investigations on the psychometric properties of the evaluated metrics within the included studies were summarized with respect to the measurement constructs and rated according to their summarized evidence. The part closes with recommendations on assessment tasks, reporting and metrics to include in post-stroke upper limb kinematic assessments.

- What are possible kinematic quantifiers of interjoint coordination and how are they expressed in different task conditions?

Upper limb movements were characterized during non-functional and functional semistructured activities of daily life in the affected and the less-affected upper limb by use of a full-body sensor-suit, based on the cross-sectional observational study (Clinicaltrials. gov.: NCT03135093, BASEC-ID: 2016-02075). Subjects in the chronic stage of at least 6-month post-stroke with mild to moderate upper limb movement deficits were included if basic grasp functions were executable. Chapter 3 focusses on the question, 
how the level upper limb interjoint coordination can be quantified across different task contents. Spatiotemporal kinematic parameters of the shoulder-elbow-trunk complex were investigated in four discrete functional and non-functional movement tasks and related to clinical measures of upper limb interjoint coordination. Chapter 4 focusses on a function-based analysis and related outcome measures of upper limb kinematic measures that were captured during a set of arm and hand activities of daily life to quantify movement complexity and the assumption of reduced movement variability in subjects after stroke.

- Considering a kinematic core-set based on the best-available evidence identified in literature, is there a difference in kinematic expressions, explained by the upper limb movement task, or the impairment level?

Chapter 5 concerns the investigation of a kinematic core-set that reflects the main domains related to spatiotemporal movement characteristics, such as speed and joint ranges, during upper limb daily living activities (gesture movements and reach-to-grasp movements). Different movement dynamics were investigated with respect to reaching and reach-to-grasp activities, and in terms of differences in the upper limb impairment level.

- Is it possible to identify comparable kinematic characterization of movement primitives or subphases across different activities of daily life?

With the aim to improve assessment possibilities in ecologically valid surroundings and task conditions, chapter 5 includes the analysis, whether motion subphases, or so-called motion primitives of reach to grasp or gesture distally and reach to transport or gesture proximally are comparable across different tasks in terms of kinematic expressions and relations.

- What are the effects of armload and target height on upper limb kinematics from the trunk to the finger digits during functional reach-to-grasp movements?

The question of effects of armload and target height on movement kinematics during functional tasks has been addressed in the third study. A pilot study has been planned and ethically approved (BASEC-No: Req-2019-00417) to investigate the feasibility of a distributed inertial sensing system including fingertip force sensing in ten subjects of at least 6 months after stroke. Chapter 6 summarizes the effects target height and/or object weight during object displacement on trunk compensation and flexion/extension of the shoulder, elbow, wrist, and finger digits. 
A brief discussion on the presented research results is followed with a focus on upper limb assessment aspects, such as the evaluated kinematic parameters, the inertial sensing systems, and the assessment protocols, in chapter 7 . The chapter finishes with the general conclusion of this thesis and suggestions on how to improve the standardization of upper limb kinematic assessments after stroke in future research and clinical settings.

\subsection{References}

Adams HP, Davis PH, Leira EC, et al. Baseline NIH Stroke Scale score strongly predicts outcome after stroke. A report of the Trial of Org 10172 in Acute Stroke Treatment (TOAST). Neurology. 1999;53:126. doi: 10.1212/WNL.53.1.126

Ahn S, Hwang S. An investigation of factors influencing the participation of stroke survivors in social and leisure activities. Phys Ther Rehabil Science. 2018;7:67-71. doi: 10.14474/ptrs.2018.7.2.67

Alawieh A, Zhao J, Feng W. Factors affecting post-stroke motor recovery: Implications on neurotherapy after brain injury. Behav Brain Res. 2018;340:94-101. doi: 10.1016/j.bbr.2016.08.029

Alexander LD, Black SE, Gao F, Szilagyi G, Danells CJ, McIlroy WE. Correlating lesion size and location to deficits after ischemic stroke: the influence of accounting for altered peri-necrotic tissue and incidental silent infarcts. Behav Brain Funct. 2010;6:6. doi: 10.1186/1744-9081-6-6

Allison R, Shenton L, Bamforth K, Kilbride C, Richards D. Incidence, Time Course and Predictors of Impairments Relating to Caring for the Profoundly Affected arm After Stroke: A Systematic Review. Physiother Res Int. 2016;21:210-27. doi: 10.1002/pri.1634

Alt Murphy M, Resteghini C, Feys P, Lamers I. An overview of systematic reviews on upper extremity outcome measures after stroke. BMC Neurol. 2015;15:29. doi: 10.1186/s12883-015-0292-6

Bamford JM. The role of the clinical examination in the subclassification of stroke. Cerebrovasc Dis. 2000;10 Suppl 4:2-4. doi: 10.1159/000047582

Bernstein N. The co-ordination and regulation of movements. Pergamon Press, Oxford; 1967.

Brott T, Adams HP Jr, Olinger CP, et al. Measurements of acute cerebral infarction: a clinical examination scale. Stroke. 1989;20:864-70.

Brunnstrom S. Motor testing procedures in hemiplegia: based on sequential recovery stages. Phys Ther. 1966;46(4):357-75. doi: 10.1093/ptj/46.4.357

Brunnstrom, S. Movement Therapy in Hemiplegia A Neurophysiological Approach. Medical Dept, Harper \& Row, New York; 1970.

Carey LM, Matyas TA, Baum C. Effects of Somatosensory Impairment on Participation After Stroke. Am J Occup Ther. 2018;72:7203205100p1-7203205100p10. doi: 10.5014/ajot.2018.025114

Cuesta-Vargas AI, Galán-Mercant A, Williams JM. The use of inertial sensors system for human motion analysis. Phys Ther Rev. 2010;15:462-73. https://doi.org/10.1179/1743288X11Y.0000000006

Dewald JP, Pope PS, Given JD, Buchanan TS, Rymer WZ. Abnormal muscle coactivation patterns during isometric torque generation at the elbow and shoulder in hemiparetic subjects. Brain. 1995;118: 495-510. doi: 10.1093/brain/118.2.495

Emos MC, Rosner J. Neuroanatomy, Upper Motor Nerve Signs. [Updated 2020 Jul 27]. In: StatPearls [Internet]. Treasure Island (FL): StatPearls Publishing; 2020 Jan-. Available from: https://www. ncbi.nlm.nih.gov/books/NBK541082/ 
Fugl-Meyer AR, Jääskö L, Leyman I, Olsson S, Steglind S. The post-stroke hemiplegic patient. 1. a method for evaluation of physical performance. Scan J Rehabil Med 1975;7:13-31.

Gladstone DJ, Danells CJ, Black SE. The fugl-meyer assessment of motor recovery after stroke: a critical review of its measurement properties. Neurorehabil Neural Repair. 2002;16:232-40. doi: $10.1177 / 154596802401105171$

Gorelick PB. The global burden of stroke: persistent and disabling. Lancet Neurol. 2019;18(5):417-8. doi: 10.1016/S1474-4422(19)30030-4

Haghi M, Thurow K, Stoll R. Wearable Devices in Medical Internet of Things: Scientific Research and Commercially Available Devices. Healthc Inform Res. 2017;23:4-15. doi: 10.4258/hir.2017.23.1.4

Held JPO, Klaassen B, Eenhoorn A, van Beijnum BF, Buurke JH, Veltink PH, Luft AR. Inertial Sensor Measurements of Upper-Limb Kinematics in Stroke Patients in Clinic and Home Environment. Front Bioeng Biotechnol. 2018;6:27. doi: 10.3389/fbioe.2018.00027

Jeannerod M. The neural and behavioral organization of goal-directed movements. Oxford: Clarendon Press; 1990.

Jørgensen HS, Nakayama H, Raaschou HO, Olsen TS. Stroke. Neurologic and functional recovery the Copenhagen Stroke Study. Phys Med Rehabil Clin N Am. 1999;10:887-906.

Klaassen B, van Beijnum BF, Held JP, Reenalda J, van Meulen FB, Veltink PH, Hermens HJ. Usability Evaluations of a Wearable Inertial Sensing System and Quality of Movement Metrics for Stroke Survivors by Care Professionals. Front Bioeng Biotechnol. 2017;5:20. doi: 10.3389/ fbioe.2017.00020

Kwakkel G, Lannin NA, Borschmann K, et al. Standardized measurement of sensorimotor recovery in stroke trials: Consensus-based core recommendations from the Stroke Recovery and Rehabilitation Roundtable. International Journal of Stroke. 2017;12:451-61. doi: 10.1177/1747493017711813

Latash ML. Motor synergies and the equilibrium-point hypothesis. Motor Control. 2010;14:294-322. doi: 10.1123/mcj.14.3.294

Lea RD, Gerhardt JJ. Range-of-motion measurements. J Bone Joint Surg Am. 1995;77:784-98. https:// doi.org/10.2106/00004623-199505000-00017

Lukos J, Ansuini C, Marco Santello M. Choice of contact points during multidigit grasping: effect of predictability of object center of mass location. J Neurosci. 2007;27:3894-903. doi: 10.1523/ JNEUROSCI.4693-06.2007

Mayer NH, Esquenazi A. Muscle overactivity and movement dysfunction in the upper motoneuron syndrome. Phys Med Rehabil Clin N Am. 2003;14:855-83, vii-viii. doi: 10.1016/s10479651(03)00093-7

McCrea PH, Eng JJ, Hodgson AJ. Saturated muscle activation contributes to compensatory reaching strategies following stroke. J Neurophysiol. 2005;94:2999-3008. doi: 10.1152/jn.00732.2004

McMorland AJC, Runnals KD, Byblow WF. A neuroanatomical framework for upper limb synergies after stroke. Front Hum Neurosci. 2015;9:82. doi: 10.3389/fnhum.2015.00082

Muller P, Begin MA, Schauer T, Seel T. Alignment-Free, Self-Calibrating Elbow Angles Measurement Using Inertial Sensors. IEEE J Biomed Health Inform. 2017;21:312-9. doi: 10.1109/ JBHI.2016.2639537

Nijland RH, van Wegen EE, Harmeling-van der Wel BC, Kwakkel G; EPOS Investigators. Presence of finger extension and shoulder abduction within 72 hours after stroke predicts functional recovery: early prediction of functional outcome after stroke: the EPOS cohort study. Stroke. 2010;41:745-50. doi: 10.1161/STROKEAHA.109.572065 
Nudo RJ. Mechanisms for recovery of motor function following cortical damage. Curr Opin Neurobiol. 2006;16:638-44. doi: 10.1016/j.conb.2006.10.004

Owens Johnson C, Nguyen M, Roth GA, et al. Global, regional, and national burden of stroke, 1990-2016: a systematic analysis for the Global Burden of Disease Study 2016. Lancet Neurol. 2019;18:439-58. doi: 10.1016/S1474-4422(19)30034-1

Paulich M., Schepers M., Rudigkeit N., Bellusci G. Xsens MTw Awinda: Miniature WirelessInertialMagnetic Motion Tracker for HighlyAccurate 3D Kinematic Applications. https://www.xsens. com/hubfs/3446270/Downloads/Manuals/MTwAwinda_WhitePaper.pdf

Persson, HC. Upper extremity functioning during the first year after stroke. Thesis. 2015.

Raghavan P. Upper Limb Motor Impairment After Stroke. Phys Med Rehabil Clin N Am. 2015;26:599610. doi: 10.1016/j.pmr.2015.06.008

Roh J, Rymer WZ, Perreault EJ, Yoo SB, Beer RF. Alterations in upper limb synergy structure in chronic stroke survivors. J Neurophysiol. 2013;109:768-81. doi: 10.1152/jn.00670.2012

Sacco RL, Kasner SE, Broderick JP, et al. An updated definition of stroke for the 21st century: a statement for healthcare professionals from the American Heart Association/American Stroke Association. Stroke. 2013;44:2064-89. doi: 10.1161/STR.0b013e318296aeca

Santello M, Flanders M, Soechting JF. Patterns of hand motion during grasping and the influence of sensory guidance. J Neurosci. 2002;22:1426-35. doi: 10.1523/JNEUROSCI.22-04-01426.2002

Santello M, Lang CE. Are movement disorders and sensorimotor injuries pathologic synergies? When normal multi-joint movement synergies become pathologic. Front Hum Neurosci. 2015;8:1050. doi: 10.3389/fnhum.2014.01050

Santisteban L, Térémetz M, Bleton JP, Baron JC, Maier MA, Lindberg PG. Upper Limb Outcome Measures Used in Stroke Rehabilitation Studies: A Systematic Literature Review. PLoS One. 2016;11:e0154792

Schepers VP, Ketelaar M, Visser-Meily AJ, de Groot V, Twisk JW, Lindeman E. Functional recovery differs between ischaemic and haemorrhagic stroke patients. J Rehabil Med. 2008;40:487-9. doi: 10.2340/16501977-0198

Shumway-Cook A, Woollacott MH. Motor Control: Translating Research into Clinical Practice. Third Edition, Lippincott Raven; 2007.

Stevens E, Emmett E, Wang Y. The burden of stroke in Europe. 2017. Accessed September 2020 on https://www.stroke.org.uk/sites/default/files/the_burden_of_stroke_in_europe_-_challenges_ for_policy_makers.pdf

Stinear CM, Barber PA, Smale PR, Coxon JP, Fleming MK, Byblow WD. Functional potential in chronic stroke patients depends on corticospinal tract integrity. Brain. 2007;130(Pt 1):170-80. doi: 10.1093/brain/awl333

Tresch MC, Jarc A. The case for and against muscle synergies. Curr Opin Neurobiol. 2009;19:601-7. doi: 10.1016/j.conb.2009.09.002

Twitchel TE. The restoration of motor function following hemiplegia in man. Brain. 1951;74:443-80. van den Noort JC. Ambulatory movement analysis systems in clinical motor function assessment: Applications of inertial sensors and an instrumented force shoe. Thesis. 2011.

van Meulen FB, Reenalda J, Buurke JH, Veltink PH. Assessment of daily-life reaching performance after stroke. Ann Biomed Eng. 2015;43:478-86. doi: 10.1007/s10439-014-1198-y 
Wafa HA, Wolfe CDA, Emmett E, Roth GA, Johnson CO, Wang Y. Burden of Stroke in Europe: ThirtyYear Projections of Incidence, Prevalence, Deaths, and Disability-Adjusted Life Years. Stroke. 2020;51:2418-27. doi: 10.1161/STROKEAHA.120.029606

Wang Q, Markopoulos P, Yu B, Chen W, Timmermans A. Interactive wearable systems for upper body rehabilitation: a systematic review. J Neuroeng Rehabil. 2017;14:20. doi: 10.1186/s12984017-0229-y

Weimar C, Kurth T, Kraywinkel K, Wagner M, Busse O, Haberl RL, Diener HC; German Stroke Data Bank Collaborators. Assessment of functioning and disability after ischemic stroke. Stroke. 2002;33:2053-9. doi: 10.1161/01.str.0000022808.21776.bf

World Health Organization. International classification of functioning, disability and health: ICF. World Health Organization; \2001. https://apps.who.int/iris/handle/10665/42407

Yao J, Chen A, Carmona C, Dewald JP. Cortical overlap of joint representations contributes to the loss of independent joint control following stroke. Neuroimage. 2009;45:490-9. doi: 10.1016/j. neuroimage.2008.12.002 



\title{
Systematic review on kinematic assessments of upper limb movements after stroke
}

\author{
A. Schwarz \\ C.M. Kanzler \\ O. Lambercy \\ A.R. Luft \\ J.M. Veerbeek
}




\subsection{Abstract}

Background and purpose - Assessing upper limb movements poststroke is crucial to monitor and understand sensorimotor recovery. Kinematic assessments are expected to enable a sensitive quantification of movement quality and distinguish between restitution and compensation. The nature and practice of these assessments are highly variable and used without knowledge of their clinimetric properties. This presents a challenge when interpreting and comparing results. The purpose of this review was to summarize the state of the art regarding kinematic upper limb assessments poststroke with respect to the assessment task, measurement system, and performance metrics with their clinimetric properties. Subsequently, we aimed to provide evidence-based recommendations for future applications of upper limb kinematics in stroke recovery research.

Methods - A systematic search was conducted in PubMed, Embase, CINAHL, and IEEE Xplore. Studies investigating clinimetric properties of applied metrics were assessed for risk of bias using the Consensus-Based Standards for the Selection of Health Measurement Instruments checklist. The quality of evidence for metrics was determined according to the Grading of Recommendations Assessment, Development, and Evaluation approach.

Results - A total of 225 studies ( $\mathrm{N}=6197)$ using 151 different kinematic metrics were identified and allocated to 5 task and 3 measurement system groups. Thirty studies investigated clinimetrics of 62 metrics: reliability $(n=8)$, measurement error $(n=5)$, convergent validity $(n=22)$, and responsiveness $(n=2)$. The metrics task/movement time, number of movement onsets, number of movement ends, path length ratio, peak velocity, number of velocity peaks, trunk displacement, and shoulder flexion/ extension received a sufficient evaluation for one clinimetric property.

Conclusions - Studies on kinematic assessments of upper limb sensorimotor function are poorly standardized and rarely investigate clinimetrics in an unbiased manner. Based on the available evidence, recommendations on the assessment task, measurement system, and performance metrics were made with the goal to increase standardization. Further highquality studies evaluating clinimetric properties are needed to validate kinematic assessments, with the long-term goal to elucidate upper limb sensorimotor recovery poststroke. 


\subsection{Introduction}

Deficits in upper limb sensorimotor function are experienced by about $80 \%$ of stroke patients early after symptom onset (Langhorne et al., 2009). Despite the availability of acute medical treatment and rehabilitation, upper limb impairment persists in about $60 \%$ of the patients six months poststroke (Nijland et al., 2010). These impairments can include muscle weakness, loss of inter-joint coordination, and changes in muscle tone and sensation, which subsequently reduce the ability to use the upper limb when performing daily activities and increase dependency (Langhorne et al., 2011; Veerbeek et al., 2011). Understanding upper limb sensorimotor recovery poststroke is required to optimize therapy outcomes by developing effective interventions. One constraint impeding this understanding is the lack of standardized and responsive approaches to define and measure stroke-related upper limb deficits and their evolution (Kwakkel et al., 2017).

Traditionally, upper limb deficits poststroke are evaluated using established clinical assessments such as the upper extremity subscale of the Fugl-Meyer Assessment (FMAUE) (Fugl-Meyer et al., 1975; Gladstone et al., 2002) and the Action Research Arm Test (ARAT) (Carroll, 1965; Lang et al., 2006). A drawback of these assessments is that they are insufficiently sensitive to capture the quality of sensorimotor performance due to the use of ordinal scales. This impedes the ability to clearly distinguish behavioral restitution from compensation (Chen et al., 2009; Lin et al., 2010), which is essential to understand neurological mechanisms of sensorimotor recovery poststroke. Behavioral restitution has been defined as "a return towards more normal patterns of motor control with the impaired effector," whereas compensation strategies include new behavioral approaches by using "intact muscles, joints and effectors in the affected limb, to accomplish the desired task or goal” (Bernhardt et al., 2017). Kinematic assessments promise to overcome these drawbacks by providing objective metrics that have the potential to sensitively capture movement quality and enable the monitoring of compensatory movements (Bernhardt et al., 2017; Krebs et al., 2014; Lambercy et al., 2016). However, a variety of tasks, measurement systems, and kinematic metrics are used in clinical research. This limits comparability between studies and the potential for meta-analyses that are needed to establish a knowledge foundation about the mechanisms of upper limb recovery. Furthermore, information about clinimetric properties such as reliability, measurement error, validity, and responsiveness of metrics derived from kinematic assessments is essential to confirm their physiological interpretation and robustness, and thereby, their suitability for stroke recovery research. 
Previous reviews summarized the use of kinematic metrics for the upper limb (de los ReyesGuzman et al., 2014; Alt Murphy and Häger, 2015; Ellis et al., 2016; Shishov et al., 2017; Wang et al., 2017; Tran et al., 2018) and their physiological interpretation (Nordin et al., 2014). However, they focused only on specific measurement systems, or did not differentiate metrics according to assessment tasks (Alt Murphy and Häger, 2015; Nordin et al., 2014); factors which are likely to influence the interpretation of kinematic metrics (Subramanian et al., 2010). In addition, the majority of these reviews were not performed in a systematic way or did not rely on guidelines such as PRISMA for reporting systematic reviews and COSMIN for assessing risk of bias and grading the evidence (Moher et al., 2009; Mokkink et al., 2018). Despite the importance of characterizing clinimetric properties, only two reviews investigated clinimetrics, but these focused solely on convergent validity between metrics and clinical scales (Tran et al., 2018), or did not consider assessment characteristics and the quality of the clinimetric evidence (Alt Murphy and Häger, 2015).

This systematic review therefore aimed to provide a complete and unbiased overview of assessment tasks, measurement systems, and metrics with their clinimetric properties (reliability, measurement error, convergent validity, and responsiveness) for kinematic upper limb assessments poststroke. Subsequently, we proposed recommendations on how to design, evaluate, and apply kinematic assessments in future stroke recovery research.

\subsection{Methods}

This systematic review was registered in PROSPERO (number CRD42017064279) and meets the Preferred Reporting Items for Systematic Reviews and Meta-Analysis (PRISMA) requirements (Moher et al., 2009). The search was performed in PubMed, Embase, CINAHL, and IEEE Xplore from inception to September 30, 2017. For the literature search in PubMed, see Supplementary Table S2.1 in the Supplementary data. The Supplementary data contains detailed information regarding eligibility criteria, information sources, study selection, and data collection. The data that support the findings of this study are available from the authors upon reasonable request.

\subsubsection{Data collection and definitions}

For each study, information about the kinematic assessment and clinimetric properties were extracted. Additionally, patient demographics, stroke-related information, and the level of upper limb impairment was recorded. 
Assessment tasks were categorized into five groups based on the nature of the performed upper limb movements. Two-dimensional (2D) tasks in the horizontal plane were divided into $2 \mathrm{D}$ pointing (i.e., discrete movements to defined targets) and 2D shape drawing (i.e., continuous movements) tasks. Three-dimensional (3D) tasks were partitioned into 3D pointing and $3 \mathrm{D}$ reach-to-grasp (i.e., discrete movements with object manipulation) tasks. Studies that could not be allocated to one of these groups were assigned to the other tasks group.

Measurement systems were categorized into three groups based on their expected influence on upper limb movements during the kinematic assessments. Influence refers especially to the interaction forces between measurement system and patient due to friction, inertia, and arm weight support. Group A contained measurement systems with minimal influence on movements, such as inertial measurement units, and optical and electromagnetic motion capture systems used without arm weight support. Group B contained measurement systems expected to have medium influence, such as end-effectors and motion capture systems used with arm weight support. Group C consists of measurement systems likely to have high influence, such as exoskeletons (Just et al., 2018).

Each reported kinematic metric (i.e., a parameter extracted from kinematic data using specific post-processing algorithms) was assigned to one of the following constructs based on their physiological interpretation: accuracy, data driven scores, efficacy, efficiency, movement planning, precision, smoothness, spatial posture, speed, temporal posture, or workspace. Their definitions (see DS) were based on previous work (Alt Murphy and Häger, 2015; Nordin et al., 2014), descriptions in the included studies, and experience of the authors and were required to link metrics to their assumed physiological interpretation.

\subsubsection{Study quality assessment}

The risk of bias for studies investigating clinimetric properties of kinematic metrics was assessed using the COnsensus-based Standards for the selection of health Measurement INstruments (COSMIN) checklist for systematic reviews (Mokkink et al., 2018). The clinimetric properties test-retest reliability (i.e., proportion of measured variance that results from actual differences between patients), measurement error (i.e., error not attributed to actual changes in the measured construct), convergent validity (i.e., degree to which correlation of metrics to clinical scales is consistent with the hypothesis), and responsiveness (i.e., ability to capture longitudinal changes in the measured construct) were analyzed. 


\subsubsection{Synthesis of results}

The results of the clinimetric evidence and study quality assessment were synthesized for each investigated metric across tasks by applying the Grading of Recommendations Assessment, Development and Evaluation (GRADE) principles (Mokkink et al., 2018). Herewith, the evidence of multiple studies is summarized based on risk of bias (i.e., study quality), inconsistency (i.e., contradicting results), and imprecision (i.e., small population sizes). For reliability, Intraclass Correlation Coefficients (ICC) of $\geq 0.7$ were considered to be "sufficient" (Just et al., 2018) (i.e., the evaluation of results was appropriate for this property). Measurement error was considered to be "sufficient", if the smallest detectable change or limits of agreement was below the minimal important change. Convergent validity was evaluated analyzing correlation coefficients $(r)$ between kinematic metrics and clinical scales. The FMA-UE was selected as reference clinical scale as it was most commonly reported for describing upper limb motor impairment (76\% of the studies). For convergent validity, a moderate to very high correlation $(|\mathrm{r}| \geq 0.5$ with $\mathrm{p} \leq 0.05)$ between the FMA-UE and all metrics describing the physiological constructs accuracy, data driven scores, efficacy, efficiency, smoothness, spatial posture, speed, temporal posture, and workspace led to a sufficient evaluation. For metrics describing another physiological construct, convergent validity could not be analyzed as it would require different reference scales that were typically not reported. For responsiveness, an area under the curve of $\geq 0.7$ was "sufficient". The evidence per clinimetric property per kinematic metric was evaluated according to the COSMIN criteria for "good measurement properties" (sufficient, insufficient, inconsistent, or indeterminate) (Mokkink et al., 2018). Outcomes were the summarized evidence (sufficient, indeterminate, or insufficient) and the quality of evidence (high, moderate, low, very low) per kinematic metric and clinimetric property. Metrics were recommended for future use if the quality of the evidence was at least moderate and the summarized evidence was sufficient.

\subsection{Results}

\subsubsection{Kinematic upper limb assessments}

The literature search resulted in 225 included studies $(\mathrm{N}=6197)$ (Figure 2.1). The included studies, as well as the participant and kinematic assessment characteristics are available upon author request. According to our task classification, 81 studies used a $2 \mathrm{D}$ pointing task, 16 a $2 \mathrm{D}$ shape drawing task, 67 a 3D pointing task, 50 a 3D reach-to-grasp task, and 24 a task belonging to the other tasks group (Supplementary Figure S2.1). Kinematic recordings 
were made with a measurement system of group A, B, and C in 130, 69, and 26 studies, respectively. In total, 151 different kinematic metrics (Figures 2.2 and 2.3; Supplementary Table S2.2) were reported to quantify upper limb sensorimotor function. Figures 2.2-2.4 provide an overview of the frequency distribution of each kinematic metric per task, the assigned physiological construct, and the reported clinimetric properties.
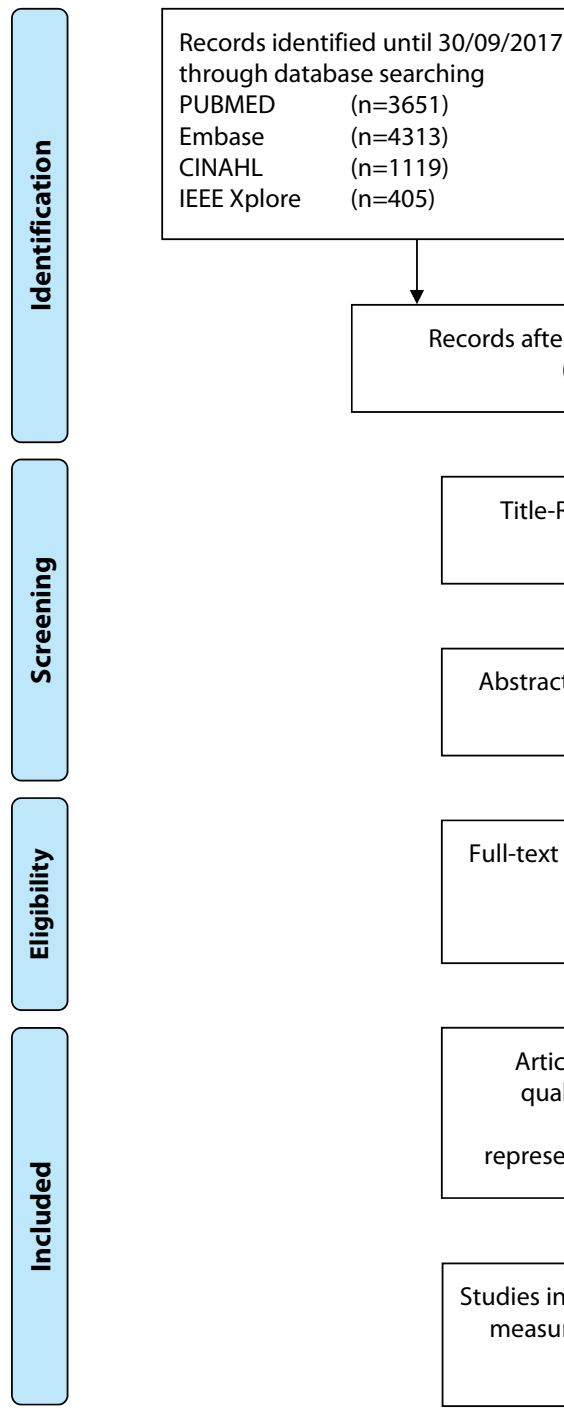

Additional records identified through other sources $(n=12)$

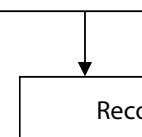

Records after duplicates removed $(n=6129)$

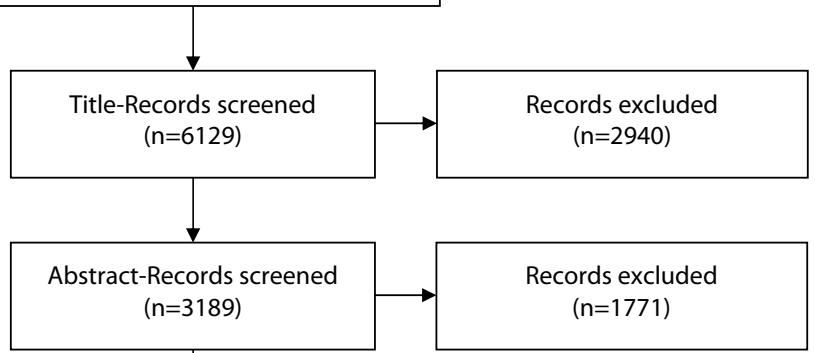

Full-text articles assessed for eligibility $(n=1418)$

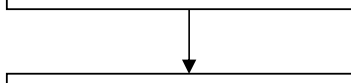

Articles included for qualitative analysis $(n=235)$

representing $\mathbf{2 2 5}$ studies

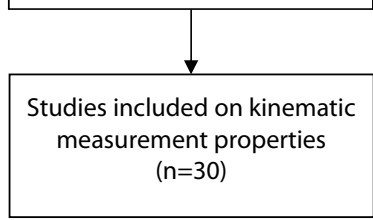

Full-text articles excluded, with reasons $(n=1190)$

- No human stroke subjects

- Stroke subject $\mathrm{N}<10$

- No patient characteristics of upper limb motor function

- Tasks involving only finger or trunk movements

- Only reporting activity counts

Figure 2.1. Preferred Reporting Items for Systematic Reviews and Meta-Analysis (PRISMA) flowchart, the systematic literature search.

Adapted from Moher et al. (2009) with permission. 


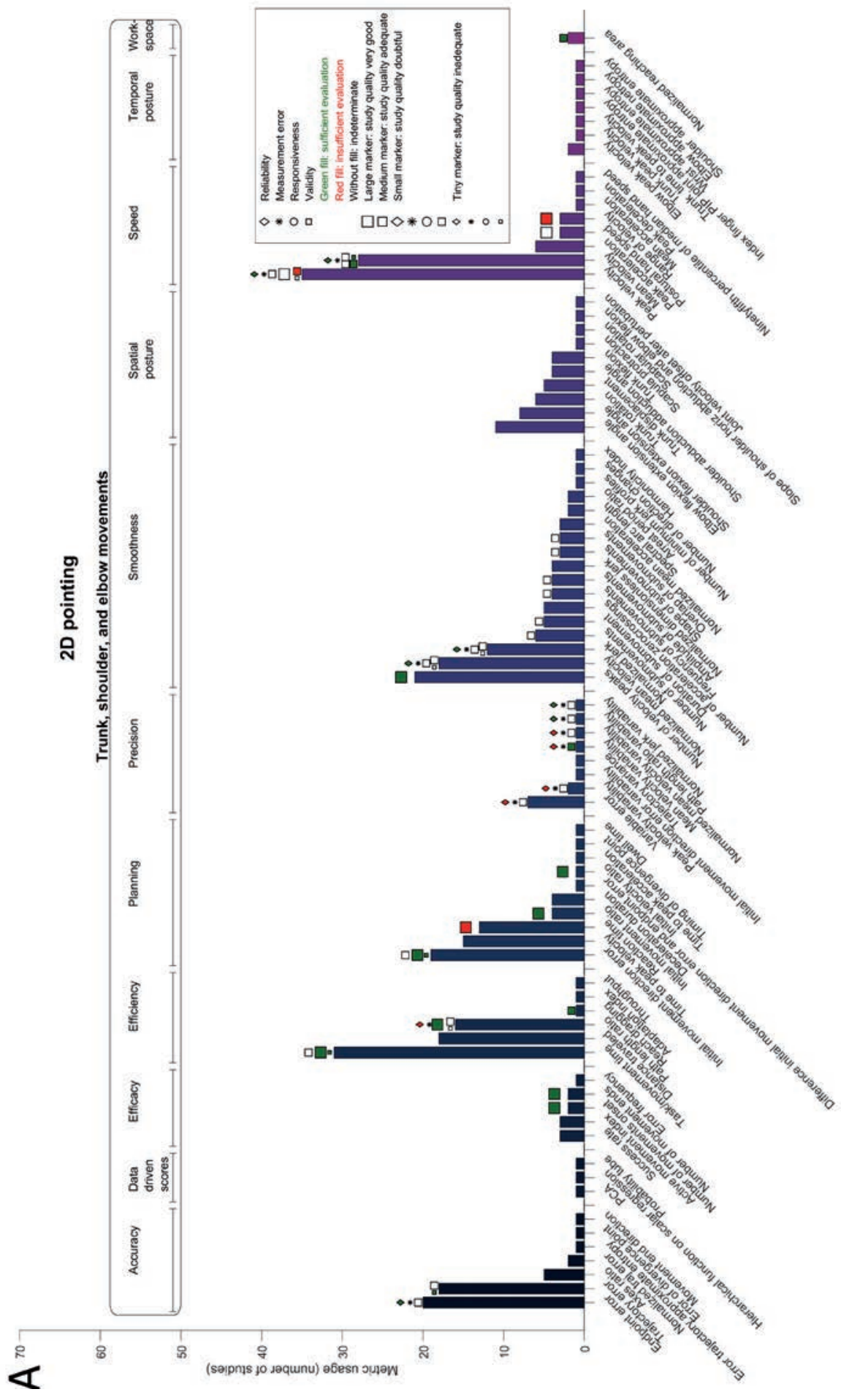



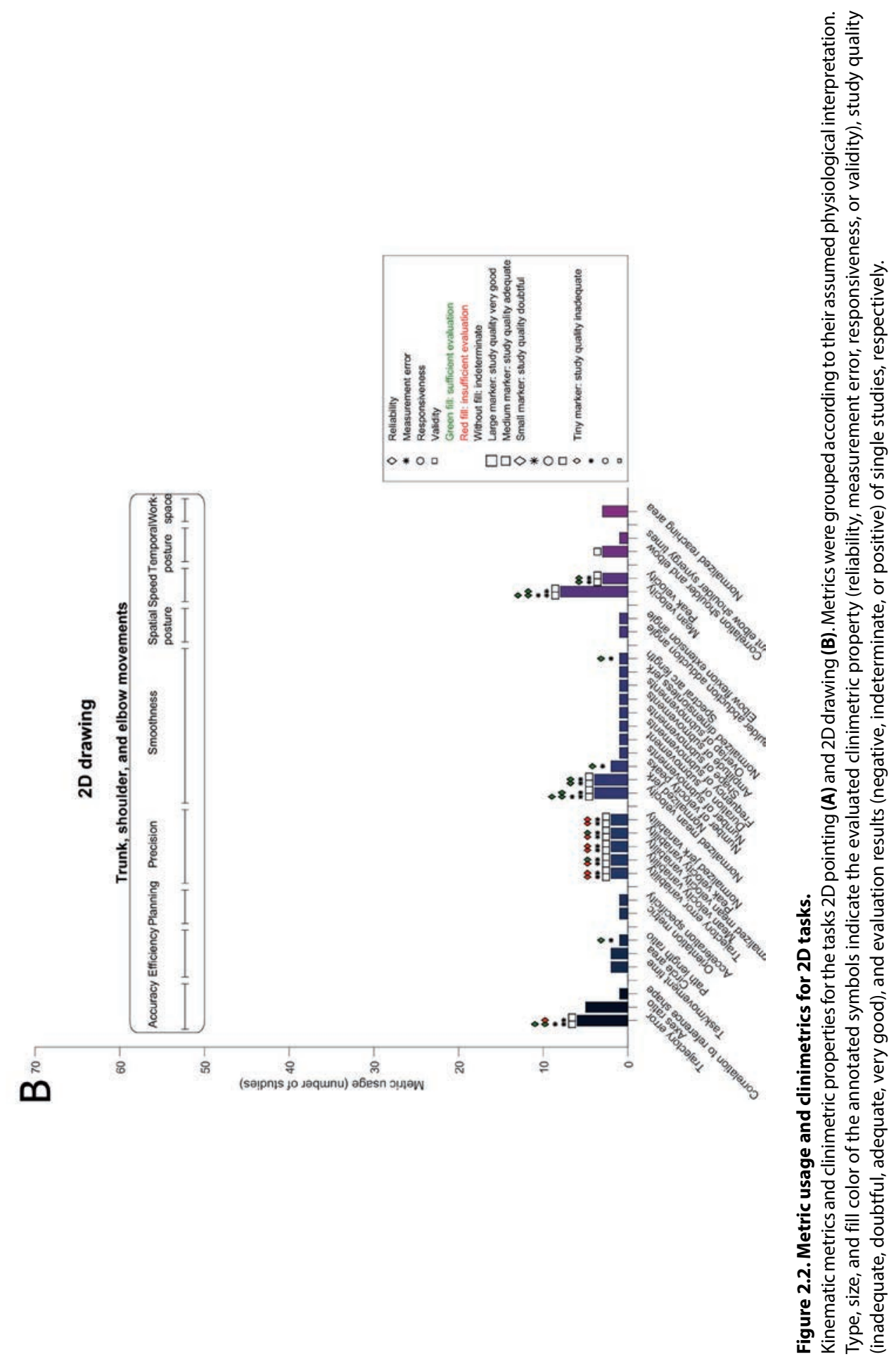

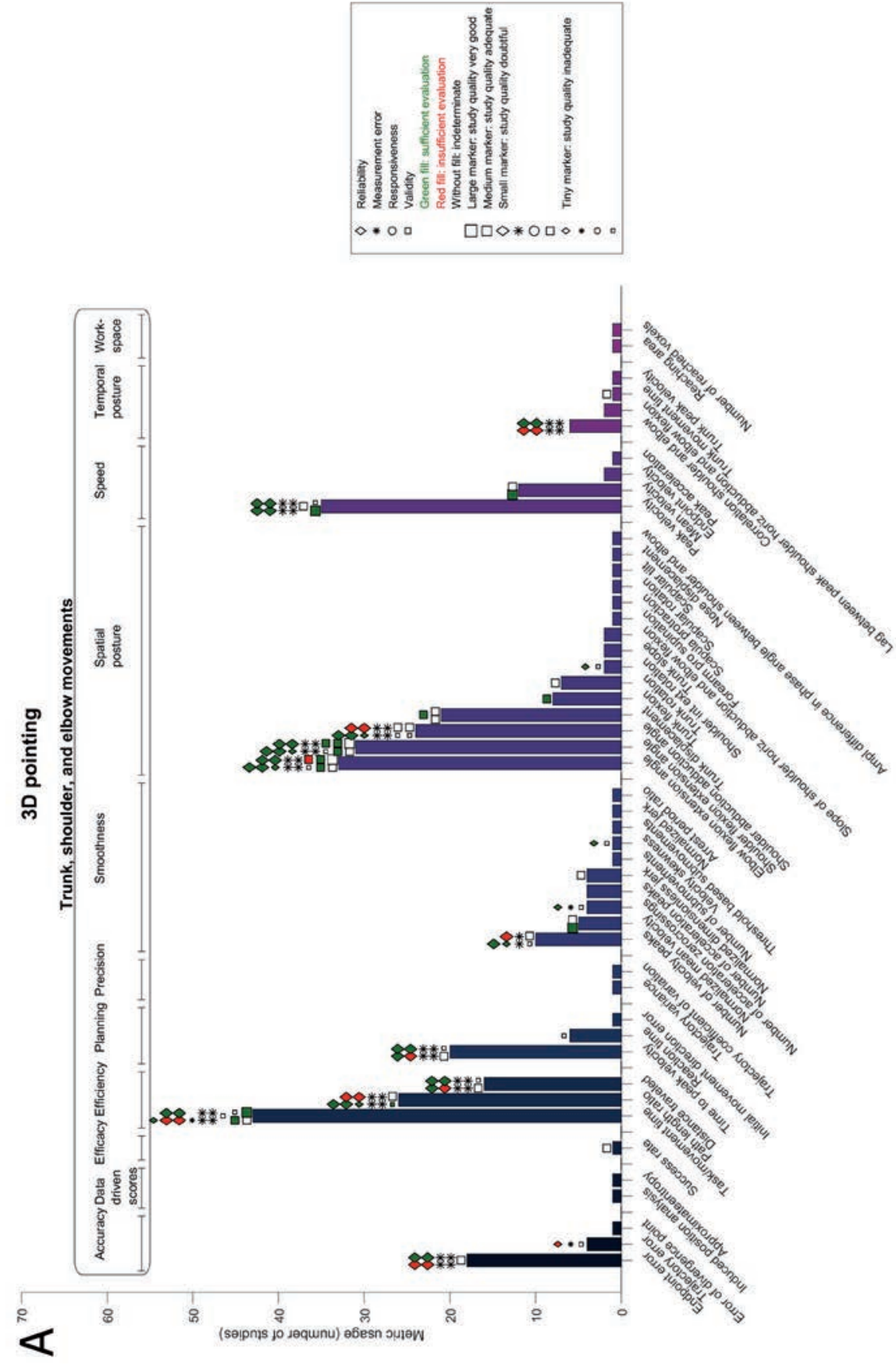


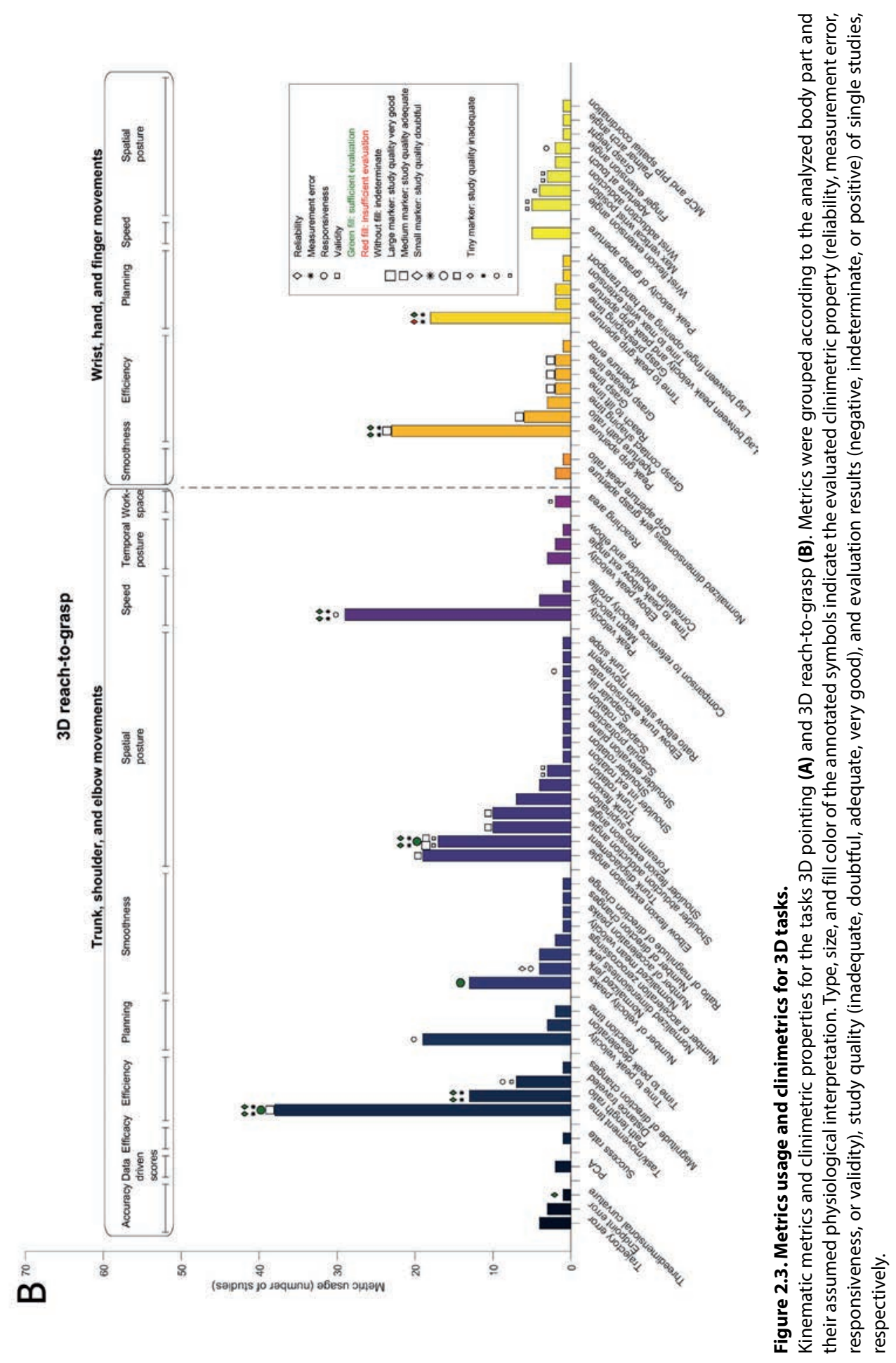




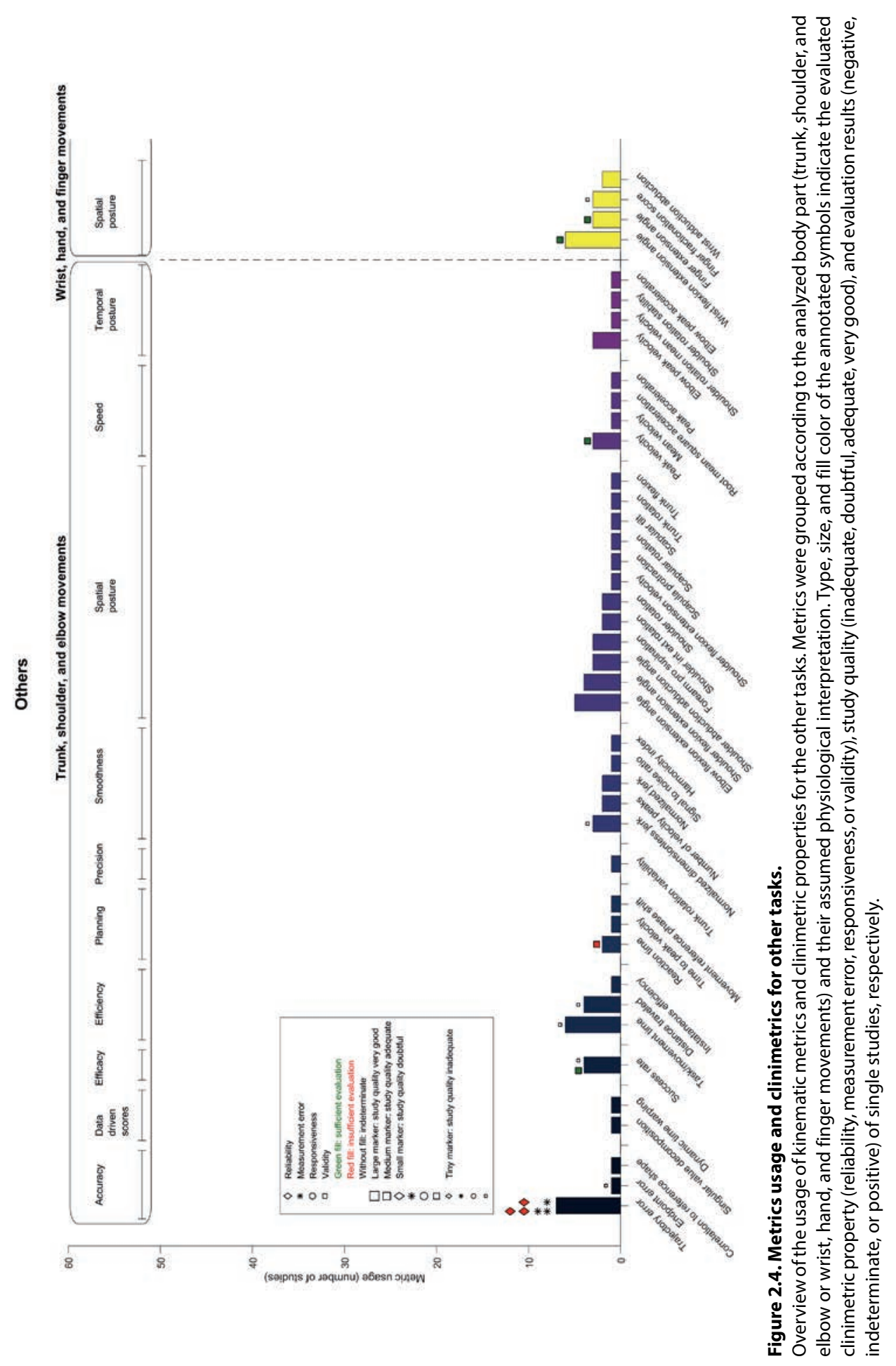




\section{D pointing tasks}

Patients $(\mathrm{N}=2536)$ included in studies using 2D pointing tasks had a median FMA-UE score of 34.35 (Interquartile Range [IQR], 22.40-47.59) (reported in n=57). Eighty-two different kinematic metrics were used, all of them describing trunk, shoulder, and elbow movements (Figure 2.2A). The five most commonly assessed physiological constructs were smoothness $(n=95)$, speed $(n=78)$, efficiency $(n=68)$, movement planning $(n=60)$, and accuracy $(n=48)$. The five most commonly used metrics were peak velocity $(n=35)$, task/movement time $(n=31)$, mean velocity $(n=28)$, number of velocity peaks $(n=21)$, and endpoint error $(n=20)$.

\section{D shape drawing tasks}

Patients $(\mathrm{N}=817)$ included in studies reporting 2D shape drawing tasks had a median FMAUE score of 33.40 (IQR, 22.00-45.69) (reported in $n=13$ ). Thirty-two different kinematic metrics were reported, all of them describing trunk, shoulder, and elbow movements (Figure 2.2B). The five most commonly assessed physiological constructs were smoothness $(n=18)$, accuracy $(n=12)$, precision $(n=12)$, speed $(n=11)$, and efficiency $(n=5)$. The five most commonly used metrics were mean velocity $(n=8)$, trajectory error $(n=6)$, axes ratio $(n=5)$, normalized mean velocity $(n=4)$, and normalized jerk $(n=4)$.

\section{D pointing tasks}

Patients $(\mathrm{N}=1818)$ included in 3D pointing tasks had a median FMA-UE score of 43.53 (IQR, 37.38-48.35) (reported in $\mathrm{n}=48$ ). Forty-nine different kinematic metrics were presented, all of them describing trunk, shoulder, and elbow movements (Figure 2.3A). The five most commonly assessed physiological constructs were spatial posture $(n=136)$, efficiency $(n=85)$, speed ( $n=50)$, smoothness $(n=32)$, and movement planning $(n=27)$. The five most commonly used metrics were task/movement time $(n=43)$, peak velocity $(n=35)$, elbow flexion/extension angle $(n=33)$, shoulder flexion/extension angle $(n=31)$, and path length ratio $(n=26)$.

\section{$3 D$ reach-to-grasp tasks}

Patients $(\mathrm{N}=1178)$ performing a 3D reach-to-grasp task had a mean FMA-UE score of 46.00 (IQR, 37.40-52.35) (reported in $\mathrm{n}=32$ ). Sixty-six different kinematic metrics were reported (Figure 2.3B). Forty-three metrics described trunk, shoulder, and elbow movements, and 23 wrist, hand, and finger movements. The five most commonly assessed physiological constructs were spatial posture $(n=79)$, efficiency $(n=59)$, grasping efficiency $(n=39)$, speed $(\mathrm{n}=34)$, and smoothness $(\mathrm{n}=27)$. The five most commonly used metrics were task/movement 
time $(n=38)$, peak velocity $(n=29)$, peak grip aperture $(n=23)$, elbow flexion/extension angle $(n=19)$, and time to peak velocity $(n=19)$.

\section{Other tasks}

Patients $(\mathrm{N}=593)$ involved in other task assessments had a mean FMA-UE score of 27.35 (IQR, 24.40-39.23) (reported in $n=6$ ). Forty-two different metrics were reported (Figure 2.4). Thirty-eight metrics described trunk, shoulder, and elbow movements and five wrist, hand, and finger movements. The five most commonly assessed physiological constructs were spatial posture $(n=25)$, spatial posture of hand, wrist, and finger $(n=14)$, efficiency $(n=11)$, accuracy $(n=9)$, and smoothness $(n=9)$. The five most commonly used metrics were trajectory error $(n=7)$, task/movement time $(n=6)$, wrist flexion/extension angle $(n=6)$, elbow flexion/extension angle $(n=5)$, and success rate $(n=4)$.

\subsubsection{Risk of bias assessment}

The results of the risk of bias assessment can be found in Supplementary Table S2.3.

\section{Synthesis of evidence for clinimetric properties}

Thirty (13.3\%) studies investigated one or more clinimetric properties of $62(41.1 \%)$ kinematic metrics. In total, 124 (20.5\%) out of 604 possible combinations of all metrics and clinimetric properties were evaluated. Table 2.1 displays the metrics/clinimetric properties with at least moderate quality of evidence and (in)sufficient summarized evidence.

\section{Test-retest reliability}

Test-retest reliability was analyzed for 30 (19.9\%) kinematic metrics. The summarized evidence was sufficient for 21 , indeterminate for two, and insufficient for seven metrics. The quality of evidence was moderate for one, low for eight, and very low for 21 metrics. The only metric with a sufficient summarized evidence and of at least moderate quality was peak velocity.

\section{Measurement error}

Measurement error was evaluated for 27 (17.9\%) kinematic metrics. The summarized evidence was indeterminate for all metrics. The quality of evidence was moderate for four, low for ten, and very low for 13 metrics. 
Table 2.1. Overview of the kinematic metrics and their clinimetric properties

\begin{tabular}{|c|c|c|c|c|}
\hline Kinematic metric & $\begin{array}{l}\text { Clinimetric } \\
\text { property }\end{array}$ & $\begin{array}{l}\text { Quality of } \\
\text { evidence }\end{array}$ & $\begin{array}{l}\text { Summarized } \\
\text { evidence }\end{array}$ & $\begin{array}{l}\text { Quantitative } \\
\text { evidence }\end{array}$ \\
\hline Number of movements onset & Validity (+) & Moderate & Sufficient & $|r|-0.54$ \\
\hline Number of movement ends & Validity (+) & Moderate & Sufficient & $|r|-0.58$ \\
\hline Task/movement time & Validity (+) & High & Sufficient & $\begin{array}{l}|r|-0.60 ;-0.60 ;-0.53 \\
-0.52\end{array}$ \\
\hline Path length ratio & Validity (+) & Moderate & Sufficient & $|r|-0.54 ; 0.85$ \\
\hline Number of velocity peaks & Validity (+) & Moderate & Sufficient & $|r|-0.58$ \\
\hline Shoulder flexion extension angle & Validity (+) & Moderate & Sufficient & $\begin{array}{l}|r| 0.50 ; 0.56 ; 0.59 ; \\
0.70\end{array}$ \\
\hline Trunk displacement & Validity (+) & Moderate & Sufficient & $|r|-0.76 ;-0.72 ;-0.68$ \\
\hline Range of velocity & Validity (-) & Moderate & Insufficient & $|r|-0.4$ \\
\hline Peak velocity & Rreliability (+) & Moderate & Sufficient & $\begin{array}{l}\text { ICC: } 0.74 ; 0.95 ; 0.74 ; \\
0.95 ; 0.87 ; 0.93 ; \\
0.87 ; 0.94 ; 0.93\end{array}$ \\
\hline
\end{tabular}

Metrics/properties are shown for which the quality of evidence (i.e., quality of the available studies) was at least moderate and the summarized evidence (i.e., quality of the clinimetric evaluation results) was either sufficient $(+)$ or insufficient (-). References can be found in the Supplementary data.

\section{Convergent validity}

Convergent validity with the FMA-UE was analyzed for 58 (38.4\%) metrics. The summarized evidence was sufficient for 22 , indeterminate for 34 , and insufficient for two metrics. The quality of evidence was high for three, moderate for 11, low for 17, and very low for 27 metrics. Metrics with a sufficient summarized evidence and of at least moderate quality were number of movement onsets/ends, task/movement time, path length ratio, number of velocity peaks, shoulder flexion/extension angle, and trunk displacement. Range of velocity was the only metric with insufficient summarized evidence and moderate quality.

\section{Responsiveness}

Responsiveness was evaluated for nine (6.0\%) metrics. The summarized evidence for responsiveness was sufficient for three and indeterminate for six metrics. The quality of evidence was very low for all metrics.

\subsection{Discussion}

This systematic review aimed to summarize the usage of tasks, measurement systems, and metrics for upper limb kinematic assessment poststroke, as well as the available evidence regarding the clinimetric properties of these metrics. We identified 225 studies, which we 
assigned to five task types and three measurement system groups. One-hundred-fifty-one kinematic metrics covering different aspects of upper limb sensorimotor function were reported. However, their clinimetric properties were only investigated in 30 studies, leading to mostly very low or low quality of evidence. Most of these studies investigated convergent validity (38.4\% of the metrics) and reliability (19.9\% of the metrics). These findings demonstrate the need for better standardization and evaluation of kinematic assessments.

There are several possible reasons for this missing standardization and clinimetric evidence. First, researchers tend to focus on the development of novel metrics rather than trying to use and validate existing ones. This may partly result from the scarce reporting on data processing methods and the dependency of some metrics on specific hardware that is not widely available. Second, systematically investigating clinimetric properties requires carefully designed studies and involves large numbers of subjects and resources (Mokkink et al., 2018), which can be challenging to provide in practice. For example, the GRADE approach of COSMIN requires evidence from at least 100 patients across studies per metric and clinimetric property to avoid downgrading the quality of evidence (Mokkink et al., 2018).

Previous reviews on upper limb kinematics have not led to specific guidelines for kinematic assessments poststroke (de los Reyes-Guzman et al., 2014; Alt Murphy and Häger, 2015; Ellis et al., 2016; Shishov et al., 2017; Wang et al., 2017; Tran et al., 2018; Nordin et al., 2014). With the aim of improving standardization, we defined evidence-based recommendations for designing and reporting kinematic upper limb assessments for stroke recovery research (Table 2.2). These should enhance comparability between studies in the future and enable statistically summarizing study results in meta-analyses. We further advocate that researchers, clinicians, and funding agencies put more effort and resources in studies focusing on the evaluation of clinimetric properties of existing kinematic metrics. These actions should enable the research community to better exploit the potential of kinematic assessments, which should help to provide objective measures characterizing components of poststroke recovery and fine-grained metrics that could better evaluate physiological changes due to rehabilitation interventions.

\subsubsection{Application of kinematic upper limb assessments}

When planning to apply upper limb kinematic assessments, it is essential to clearly define research questions and hypotheses that should guide the choice of task type, measurement system, and metrics. For example, in order to assess possible compensatory movement 
strategies, a 3D movement task requiring the coordination of shoulder abduction, elbow extension, and hand opening and closing (e.g., 3D reach-to-grasp) should be favored, as these movements are known to elicit pathological synergy patterns (Kwakkel et al., 2017; Bernhardt

Table 2.2. Recommendations for kinematic upper limb assessments poststroke

\begin{tabular}{ll}
\hline $\begin{array}{l}\text { Assessment } \\
\text { task }\end{array}$ & - Should be hypothesis driven - task follows a research question \\
& Should correspond to the physical capabilities of the patient population \\
& - SMA-UE voluntary upper limb movement, but FMA-UE <30: 2D task \\
& - Should, in case of an intervention trial, not coincide with the therapy task
\end{tabular}

Kinematic metrics*

- Should be hypothesis driven - metrics selection follows a research question and corresponds to physiological constructs of interest

- Should be selected based on available clinimetric evidence, the frequency of use, and insights from motor control, technical, and clinical perspectives:

\section{Trunk/shoulder/elbow movements}

- accuracy: trajectory error, endpoint error

- efficacy: number of movement onsets, number of movement ends, success rate

- efficiency: task/movement time, path length ratio, distance traveled

- planning: time to peak velocity, reaction time, initial movement direction error

- precision: variable error

- smoothness: number of velocity peaks, normalized dimensionless jerk, spectral arc length

- spatial posture: trunk displacement, shoulder flexion/extension angle, shoulder abduction/adduction angle, elbow flexion/extension angle

- speed: peak velocity

- temporal posture: elbow peak velocity, time to peak elbow extension angle, correlation shoulder and elbow, trunk movement time, trunk peak velocity

- workspace: normalized reaching area

\section{Wrist/hand/finger movements}

- accuracy:-

- efficacy:-

- efficiency: peak grip aperture, aperture path ratio, grasp time, grasp release time

- planning: time to peak grip aperture

- precision:-

- smoothness: normalized dimensionless jerk grasp aperture

- spatial posture: wrist flexion/extension angle, maximal vertical wrist position, wrist adduction/abduction angle, finger extension angle

- speed: peak velocity of grasp aperture

Reporting - Research questions, hypotheses, patient population, task, measurement system, kinematic metrics, and positioning/instructions of the subject should be described; implementation of metrics should be transparent (equations and processing steps)

Methodology - Evaluating clinimetric properties is urgently needed and should be performed according to standardized guidelines (e.g., COSMIN)

Bold entries indicate metrics with at least moderate quality of evidence and sufficient summarized evidence in one clinimetric property; -, no recommendation could be made; COSMIN, COnsensus-based Standards for the selection of health Measurement INstruments. 
et al., 2017; Krebs et al., 2014). Compensatory movements can then be quantitatively captured by kinematic parameters describing the spatial posture of trunk, shoulder, elbow, and hand as well as the efficiency of movements. Hence, a set of metrics including trunk displacement, shoulder flexion/extension angle, shoulder abduction/adduction angle, elbow flexion/extension angle, wrist flexion/extension angle, and path length ratio might be appropriate to capture these movements patterns (Bernhardt et al., 2017), as reflected by their frequent application in 3D tasks (Figure 2.3). This is also supported by the moderateto-high correlation coefficients between most of these metrics and the FMA-UE, a clinical measure of pathological joint coupling, reported in this (Table 2.2) and previous reviews (Alt Murphy and Häger, 2015; Tran et al., 2018). Furthermore, the selected assessment task should be self-contained. Tasks trained during therapy, such as robot-assisted therapy, should be avoided, as they confound results about upper limb function by including taskspecific learning effects (Schweighofer et al., 2018). Data collected within therapy sessions should be exclusively used for monitoring performance during therapy and automatically adapting the difficulty level (Metzger et al., 2015). Additionally, the measurement system should have limited influence on the performed movements to best capture patient behavior during the task. For example, the inertia and arm weight support of an active exoskeleton could influence the synergistic coupling of the shoulder, elbow, and hand, thereby affecting the validity of kinematic outcomes (Ellis et al., 2016; Lan et al., 2017; Kanzler et al., 2018).

For assessing quality of sensorimotor performance, recommendations are proposed in Table 2.2 to guide the choice of metrics based on the available clinimetric evidence, the frequency of use, and insights from motor control, technical, and clinical perspectives. The clinimetric evidence could only rarely be used as a single criterion for the recommendations due to the mostly very low and low quality of the evidence. This further underlines the need for systematically evaluating clinimetric properties. Hence, it is of high priority that kinematic metrics that are often reported in the literature (Figures 2.2-2.4), but poorly described in terms of clinimetric properties, are evaluated. This knowledge could help to better establish kinematic upper limb assessments poststroke.

Lastly, we want to emphasize the importance of reporting and discussing the influence of task type, measurement system, and task context (i.e., therapy task or self-contained assessment task) on the results, as these factors can challenge the comparison of metrics across studies. We further recommend that researchers report definitions of kinematic metrics, including equations, targeted physiological construct, signal processing methods, and clinimetric evidence, to foster transparency and thereby standardization. 


\subsubsection{Evaluation of clinimetric properties}

For the first time, a methodological approach was applied for systematically investigating clinimetric properties of kinematic upper limb assessments. This allowed identifying misconceptions in study design and execution for most scientific publications, which led to low study quality according to COSMIN standards (Mokkink et al., 2018). Hence, we recommend that researchers design and report clinimetric studies according to standardized guidelines like COSMIN. Nevertheless, the evaluation of some clinimetric properties remains challenging. For example, the comparison between clinical assessments and kinematic measures for analyzing convergent validity requires the choice of clinical assessments that capture the content of the physiological construct described by the kinematic metrics. This can be complex due to the often unclear relationships between clinical and kinematic assessments and the high amount of resources required to apply a battery of these assessments (McKenzie et al., 2017). It nevertheless seems to be inadequate to expect very high correlations between kinematic metrics and clinical scales. Kinematic assessments are assumed to provide sensitive and obective readouts without ceiling effects (Thrane et al., 2018), while clinical scales are mostly of ordinal nature with low resolution and often have ceiling effects (Gladstone et al., 2002). We can expect that kinematic assessments provide complementary information to clinical scales, which might lead to lower convergent validity. The evaluation of other aspects of validity, such as the comparison between stroke patients and healthy age-matched controls (i.e., discriminative or known group validity (Mokkink et al., 2018)) should therefore also be considered (Alt Murphy and Häger, 2015).

Acknowledging the relevance of reliability and responsiveness when investigating physiologically relevant changes during recovery, it is of utmost importance that more effort will be put into increasing evidence-based evaluations of these clinimetric properties.

\subsubsection{Limitations}

We suggested a classification for assessment tasks based on the nature of the performed movements, for measurement systems based on their influence on movements, and for metrics based on the assumed physiological interpretation. We acknowledge that this classification could have been implemented differently, although our suggestions were based on the literature, descriptions provided by the studies, and experience of the authors. In addition, the convergent validity analysis was conducted solely by comparing kinematic metrics and the FMA-UE, because other clinical assessments were not consistently reported. 


\subsection{Conclusion}

Although upper limb kinematic assessments are frequently used in stroke research, there is a lack of standardization for the use of assessment tasks, measurement systems, and kinematic metrics, as well as a paucity of high-quality studies analyzing clinimetrics. We underlined important considerations and proposed recommendations for designing and reporting of kinematic assessments after stroke, as well as for performing studies to evaluate clinimetric properties. These recommendations aim to enhance standardized and evidencebased kinematic upper limb assessments, with the long-term goal to elucidate upper limb recovery poststroke.

\subsection{References}

Alt Murphy M, Häger CK. Kinematic analysis of the upper extremity after stroke - how far have we reached and what have we grasped? Phys Ther Rev. 2015;20:137-55.

Bernhardt J, Hayward KS, Kwakkel G, et al. Agreed definitions and a shared vision for new standards in stroke recovery research: The stroke recovery and rehabilitation roundtable taskforce. Int J Stroke. 2017;12:444-50.

Carroll D. A quantitative test of upper extremity function. J Chronic Dis. 1965;18:479-91.

Chen HM, Chen CC, Hsueh IP, Huang SL, Hsieh CL. Test-retest reproducibility and smallest real difference of 5 hand function tests in patients with stroke. Neurorehabil Neural Repair. 2009;23:435-40.

de los Reyes-Guzman A, Dimbwadyo-Terrer I, Trincado-Alonso F, Monasterio-Huelin F, Torricelli D, Gil-Agudo A. Quantitative assessment based on kinematic measures of functional impairments during upper extremity movements: A review. Clin Biomech. 2014;29:719-27.

Ellis MD, Lan Y, Yao J, Dewald JPA. Robotic quantification of upper extremity loss of independent joint control or flexion synergy in individuals with hemiparetic stroke: A review of paradigms addressing the effects of shoulder abduction loading. J Neuroeng Rehabil. 2016;13:95.

Fugl-Meyer AR, Jaasko L, Norlin V. The post-stroke hemiplegic patient. Ii. Incidence, mortality, and vocational return in Goteborg, Sweden with a review of the literature. Scand J Rehabil Med. 1975;7:73-83.

Gladstone DJ, Danells CJ, Black SE. The Fugl-Meyer assessment of motor recovery after stroke: A critical review of its measurement properties. Neurorehabil Neural Repair. 2002;16:232-40.

Just F, Özen Ö, Bösch P, et al. Exoskeleton transparency across velocities: Feed-forward compensation vs. Disturbance observer. Automatisierungstechnik. 2018;66:1014-26. doi: https://doi.org/10.1515/ auto-2018-0069

Kanzler C, Martinez Gomez S, Rinderknecht M, Gassert R, Lambercy O. Influence of arm weight support on a robotic assessment of upper limb function. 7th IEEE RAS/EMBS Int Conf Biomed Rob Biomechatron; 2018 (pp. 1-6).

Krebs HI, Krams M, Agrafiotis DK, et al. Robotic measurement of arm movements after stroke establishes biomarkers of motor recovery. Stroke. 2014;45:200-4. 
Kwakkel G, Lannin NA, Borschmann K, et al. Standardized measurement of sensorimotor recovery in stroke trials: Consensus-based core recommendations from the stroke recovery and rehabilitation roundtable. Neurorehabil Neural Repair. 2017;31:784-92.

Lambercy O, Maggioni S, Luenenburger L, Gassert R, Bolliger M. Neurorehabilitation technology. Robots and wearable sensor technologies for measurement/clinical assessment. 2nd Ed. Springer Verlag Berlin; 2016 (pp. 183-207).

Lan Y, Yao J, Dewald JPA. The impact of shoulder abduction loading on volitional hand opening and grasping in chronic hemiparetic stroke. Neurorehabil Neural Repair. 2017;31:521-9.

Lang CE, Wagner JM, Dromerick AW, Edwards DF. Measurement of upper-extremity function early after stroke: Properties of the Action Research Arm Test. Arch Phys Med Rehabil. 2006;87:160510.

Langhorne P, Bernhardt J, Kwakkel G. Stroke rehabilitation. Lancet. 2011;377:1693-702.

Langhorne P, Coupar F, Pollock A. Motor recovery after stroke: A systematic review. Lancet Neurol. 2009;8:741-54.

Lin KC, Chuang LL, Wu CY, Hsieh YW, Chang WY. Responsiveness and validity of three dexterous function measures in stroke rehabilitation. J Rehabil Res Dev. 2010;47:563-71.

McKenzie A, Dodakian L, See J, et al. Validity of robot-based assessments of upper extremity function. Arch Phys Med Rehabil. 2017;98:1969-76.e2.

Metzger J, Lambercy O, Gassert R. Performance comparison of interaction control strategies on a hand rehabilitation robot. IEEE Int Conf Rehabil Rob. 2015:846-51.

Moher D, Liberati A, Tetzlaff J, Altman DG. Preferred reporting items for systematic reviews and meta-analyses: The PRISMA statement. J Clin Epidemiol. 2009;62:1006-12.

Mokkink LB, de Vet HCW, Prinsen CAC, et al. COSMIN risk of bias checklist for systematic reviews of patient-reported outcome measures. Qual Life Res. 2018;27:1171-9.

Nijland RH, van Wegen EE, Harmeling-van der Wel BC, Kwakkel G. Presence of finger extension and shoulder abduction within 72 hours after stroke predicts functional recovery: Early prediction of functional outcome after stroke: The epos cohort study. Stroke. 2010;41:745-50.

Nordin N, Xie SQ, Wünsche B. Assessment of movement quality in robot- assisted upper limb rehabilitation after stroke: A review. J Neuroeng Rehabil. 2014;11:137.

Schweighofer N, Wang C, Mottet D, et al. Dissociating motor learning from recovery in exoskeleton training post-stroke. J Neuroeng Rehabil. 2018;15:89.

Shishov N, Melzer I, Bar-Haim S. Parameters and measures in assessment of motor learning in neurorehabilitation; a systematic review of the literature. Front Hum Neurosci. 2017;11:82.

Subramanian SK, Yamanaka J, Chilingaryan G, Levin MF. Validity of movement pattern kinematics as measures of arm motor impairment poststroke. Stroke. 2010;41:2303-8.

Thrane G, Alt Murphy M, Sunnerhagen KS. Recovery of kinematic arm function in well-performing people with subacute stroke: A longitudinal cohort study. J Neuroeng Rehabil. 2018;15:67.

Tran VD, Dario P, Mazzoleni S. Kinematic measures for upper limb robot-assisted therapy following stroke and correlations with clinical outcome measures: A review. Med Eng Phys. 2018;53:13-31.

Veerbeek JM, Kwakkel G, van Wegen EE, Ket JC, Heymans MW. Early prediction of outcome of activities of daily living after stroke: A systematic review. Stroke. 2011;42:1482-8.

Wang Q, Markopoulos P, Yu B, Chen W, Timmermans A. Interactive wearable systems for upper body rehabilitation: A systematic review. J Neuroeng Rehabil. 2017;14:20. 


\subsection{Supplementary data}

\section{MATERIAL AND METHODS}

\section{1a. Definitions}

Stroke was defined as "a central nervous system infarction attributable to ischemia or hemorrhage based on neuropathological, neuroimaging, and/or clinical evidence of permanent injury that incorporates specific clinical and tissue criteria". ${ }^{1}$ The time poststroke was defined as hyper-acute ( 0 to 24 hours), acute ( 1 to 7 days), early subacute ( 7 days to 3 months), late subacute ( 3 to 6 months), and chronic (>6 months). ${ }^{2}$

\section{1b. Eligibility criteria}

This review included studies on stroke subjects of 18 years or older. There were no restrictions in terms of timing poststroke or stroke severity. Cohort studies and randomized controlled trials ( $\geq 10$ participants) applying upper limb kinematic assessments and at least one clinical measure of upper limb motor function and/or capacity were included. Publications in peerreviewed journals written in the English, German, French, Dutch, or Spanish language were considered for inclusion. Identified hits were excluded, if the task involved solely trunk or finger movements. Studies that were limited to quantifying acceleration data into activity counts were excluded. Kinematic measures based on passive motions for assessing proprioception or hyperreflexia were also deemed for exclusion.

\section{1c. Information sources and search}

A systematic search in the following electronic databases was performed (from inception until 30/09/2017): PubMed, EMBASE, CINAHL, and IEEE Xplore. 'Stroke' and 'upper limb' and 'kinematics' were the key words searched for by using MeSH terms and free text words with synonyms and related terms in title and abstract. See Supplementary Table S2.1 for the search strategy as applied in PubMed. The search strings for the other databases can be obtained by the corresponding author.

\section{1d. Study selection}

Two unblinded reviewers screened all hits (AS and CMK). The first 1500 hits were searched independently and after crosschecking search findings, the rest of the hits were searched by one reviewer (AS or CMK) and crosschecked in case of uncertainty with the other reviewer. Disagreement was solved by involving a third reviewer (JMV) to reach consensus. 


\section{Supplementary Table S2.1. Literature search strategy in PubMed}

\#6 Search $(((()(()((($ (Stroke"[Mesh] OR cva[tiab] OR cvas[tiab] OR poststroke*[tiab] OR stroke*[tiab] OR apoplex*[tiab])) OR ((brain*[tiab] OR cerebr*[tiab] OR cerebell*[tiab] OR intracran*[tiab] OR intracerebral*[tiab] OR vertebrobasilar*[tiab]) AND vascular*[tiab] AND (disease[tiab] OR diseases[tiab] OR accident*[tiab] OR disorder*[tiab]))) OR (cerebrovascular*[tiab] AND (disease[tiab] OR diseases[tiab] OR accident*[tiab] OR disorder*[tiab]))) OR ((brain*[tiab] OR cerebr*[tiab] OR cerebell*[tiab] OR intracran*[tiab] OR intracerebral*[tiab] OR vertebrobasilar*[tiab]) AND (haemorrhag*[tiab] OR hemorrhag*[tiab] OR ischemi*[tiab] OR ischaemi*[tiab] OR infarct*[tiab] OR haematoma*[tiab] OR hematoma*[tiab] OR bleed*[tiab]))) OR (("Hemiplegia"[Mesh] OR "Paresis"[Mesh] OR hemipleg*[tiab] OR hemipar*[tiab] OR paresis[tiab] OR paretic[tiab]))))) AND $(((((()(((()(((($ "Upper Extremity"[Mesh]) OR "Upper Extremity/ physiopathology"[Mesh]) OR "Upper Extremity"[Mesh]) OR "Upper Extremity/physiology"[Mesh]) OR "Arm/ physiopathology"[Mesh]) AND upper extremit*[tiab]) OR upper limb*[tiab]) OR arm[tiab]) OR arms[tiab]) OR shoulder*[tiab]) OR elbow*[tiab]) OR forearm*[tiab]) OR wrist*[tiab]) OR hand[tiab]) OR hands[tiab])))) AND $(((()((()((()((($ trajector* $[$ tiab]) OR smoothness[tiab]) OR velocit*[tiab]) OR jerk[tiab]) OR accuracy[tiab]) OR coordin*[tiab]) OR synerg*[tiab]) OR reach*[tiab]) OR grasp*[tiab]) OR grip*[tiab]) OR (movement[tiab] AND (qualit*[tiab]))))) OR kinemat*[tiab])))) NOT (((“cerebral palsy"[tiab] OR pediatric*[tiab] OR child*[tiab] OR "cerebral palsy"[Mesh])))

\#5 Search (("cerebral palsy"[tiab] OR pediatric*[tiab] OR child*[tiab] OR "cerebral palsy"[Mesh]))

\#4 Search $(((()((((" S t r o k e "[M e s h]$ OR cva[tiab] OR cvas[tiab] OR poststroke*[tiab] OR stroke*[tiab] OR apoplex*[tiab])) OR ((brain*[tiab] OR cerebr*[tiab] OR cerebell*[tiab] OR intracran*[tiab] OR intracerebral*[tiab] OR vertebrobasilar*[tiab]) AND vascular*[tiab] AND (disease[tiab] OR diseases[tiab] OR accident*[tiab] OR disorder*[tiab]))) OR (cerebrovascular*[tiab] AND (disease[tiab] OR diseases[tiab] OR accident*[tiab] OR disorder*[tiab]))) OR ((brain*[tiab] OR cerebr*[tiab] OR cerebell*[tiab] OR intracran*[tiab] OR intracerebral*[tiab] OR vertebrobasilar*[tiab]) AND (haemorrhag*[tiab] OR hemorrhag*[tiab] OR ischemi*[tiab] OR ischaemi*[tiab] OR infarct*[tiab] OR haematoma*[tiab] OR hematoma*[tiab] OR bleed*[tiab]))) OR (("Hemiplegia"[Mesh] OR "Paresis"[Mesh] OR hemipleg*[tiab] OR hemipar*[tiab] OR paresis[tiab] OR paretic[tiab]))))) AND $(((((()(((()(((("$ Upper Extremity"[Mesh]) OR "Upper Extremity/ physiopathology"[Mesh]) OR "Upper Extremity"[Mesh]) OR "Upper Extremity/physiology"[Mesh]) OR "Arm/ physiopathology"[Mesh]) AND upper extremit*[tiab]) OR upper limb*[tiab]) OR arm[tiab]) OR arms[tiab]) OR shoulder*[tiab]) OR elbow*[tiab]) OR forearm*[tiab]) OR wrist*[tiab]) OR hand[tiab]) OR hands[tiab])))) AND $(((()((()((()(($ trajector*[tiab]) OR smoothness[tiab]) OR velocit*[tiab]) OR jerk[tiab]) OR accuracy[tiab]) OR coordin*[tiab]) OR synerg*[tiab]) OR reach*[tiab]) OR grasp*[tiab]) OR grip*[tiab]) OR (movement[tiab] AND (qualit*[tiab]))))) OR kinemat*[tiab])))

\#3 Search $(((()((()((()(($ trajector*[tiab]) OR smoothness[tiab]) OR velocit*[tiab]) OR jerk[tiab]) OR accuracy[tiab]) OR coordin*[tiab]) OR synerg*[tiab]) OR reach*[tiab]) OR grasp*[tiab]) OR grip*[tiab]) OR (movement[tiab] AND (qualit*[tiab]))))) OR kinemat*[tiab]))

\#2 Search $((()((()(()((() " U p p e r$ Extremity"[Mesh]) OR "Upper Extremity/physiopathology"[Mesh]) OR "Upper Extremity"[Mesh]) OR "Upper Extremity/physiology"[Mesh]) OR "Arm/physiopathology"[Mesh]) AND upper extremit*[tiab]) OR upper limb*[tiab]) OR arm[tiab]) OR arms[tiab]) OR shoulder*[tiab]) OR elbow*[tiab]) OR forearm*[tiab]) OR wrist*[tiab]) OR hand[tiab]) OR hands[tiab]))

\#1 Search $(((((($ "Stroke"[Mesh] OR cva[tiab] OR cvas[tiab] OR poststroke*[tiab] OR stroke*[tiab] OR apoplex*[tiab])) OR ((brain*[tiab] OR cerebr*[tiab] OR cerebell*[tiab] OR intracran*[tiab] OR intracerebral*[tiab] OR vertebrobasilar*[tiab]) AND vascular*[tiab] AND (disease[tiab] OR diseases[tiab] OR accident*[tiab] OR disorder*[tiab]))) OR (cerebrovascular*[tiab] AND (disease[tiab] OR diseases[tiab] OR accident*[tiab] OR disorder*[tiab]))) OR ((brain*[tiab] OR cerebr*[tiab] OR cerebell*[tiab] OR intracran*[tiab] OR intracerebral*[tiab] OR vertebrobasilar*[tiab]) AND (haemorrhag*[tiab] OR hemorrhag*[tiab] OR ischemi*[tiab] OR ischaemi*[tiab] OR infarct*[tiab] OR haematoma*[tiab] OR hematoma*[tiab] OR bleed*[tiab]))) OR (("Hemiplegia"[Mesh] OR "Paresis"[Mesh] OR hemipleg*[tiab] OR hemipar*[tiab] OR paresis[tiab] OR paretic[tiab])))) 


\section{1e. Data collection}

A data collection form was designed and pilot tested by two reviewers (AS and CMK) and agreed on by three reviewers (AS, CMK, JMV). The form contained information regarding the first author; publication year; number of stroke participants; gender distribution; age; type of stroke (ischemic/hemorrhagic); brain lesion side (left/right); time poststroke; upper limb motor function; and applied standard clinical assessments. Extracted information regarding upper limb kinematic assessments were the type of task, including additional information regarding back support, trunk restrictions, and anti-gravity support; the measurement system; and used metrics. Based on their physiological interpretation, each kinematic metric was assigned to one of the following eleven categories.

Accuracy describes the spatial error of movements relative to optimal behavior (e.g., relative to a straight line). Data driven scores apply statistical transformations on either sensor data or metrics to construct an abstract score that is not intuitively relatable to physiological behavior (e.g., principal component analysis). Efficacy describes if a targeted task goal could be successfully achieved or not. Efficiency defines the quality of how a targeted task goal was reached. Movement planning captures the ability to perform goal-directed in a feedforward manner. Precision is the variability of performance across multiple repetitions of the same task. Smoothness analyzes the quality of feedforward control based on the deviation of the velocity profile to an optimal, bell-shaped velocity profile. Spatial posture describes position-related aspects of single or multiple joints. Speed refers to how fast movements were performed. Temporal posture describes time-related aspects of single or multiple joints. Workspace is related to the maximally reachable area or volume with a specific joint.

One reviewer (AS) extracted three-quarters of the study information and a second reviewer (CMK) extracted one-quarter. Reviewers were not blinded. Three reviewers (AS, CMK, JMV) crosschecked comprehensiveness and correctness of the extracted data.

Missing information on participant characteristics such as Fugl-Meyer Assessment - Upper Extremity (FMA-UE) subscale scores and stroke information were requested via email for 18 studies. Five authors provided additional information..$^{3-7}$

\section{1f. Risk of bias assessment}

The methodological quality of the included studies investigating measurement properties was assessed using the COSMIN Risk of Bias checklist for systematic reviews ${ }^{8}$ by two reviewers independently (AS and JMV). Disagreements were solved by discussion. For 
the present review, the Boxes 6 - Reliability (8 items), 7 - Measurement Error (6 items), $9 \mathrm{a}$ - Hypothesis testing/ convergent validity, comparison with another outcome (4 items) and 10b - Responsiveness - construct approach (4 items) were applicable. The items were rated on a four-point rating scale as 'very good', 'adequate', 'doubtful', or 'inadequate.' The overall quality of the study was based on the lowest score for that particular Box. ${ }^{8}$ Prior to assessing the included studies, pilot testing of the relevant boxes took place on three exemplary publications.

\section{1g. Synthesis of results}

Descriptive statistics regarding patient characteristics, task characteristics, and usage of kinematic parameters were applied. Each kinematic metric was categorized according to the body part targeted (arm movements, i.e. proximal part of the upper extremity; or hand movements, i.e. distal part of the upper extremity) and its physiological interpretation (accuracy, data driven scores, efficacy, efficiency, planning, precision, smoothness, spatial posture, speed, temporal posture, workspace) by four reviewers independently (AS, CMK, OL, JMV). Consensus regarding the categorization was reached through discussion. The risk of bias of the individual studies was used for grading the evidence (see Synthesis of results in the main text).

\section{RESULTS}

The search revealed 235 relevant hits, of which ten ${ }^{10-19}$ presented data that were previously published and already included in the review. Therefore, the search resulted in a total of 225 unique studies. . $^{3-70-236}$

\section{2a. Participant characteristics}

Stroke survivors were on average $59.2 \pm 11$ years old and were included in the acute stage in five studies, ${ }^{20-24}$ in the early subacute stage in 35 studies, ${ }^{7,25-58}$ in the late subacute stage in seven studies, ${ }^{59-65}$ and in the chronic stage in 172 studies $^{3-6,66-232}$ and not reported in four studies. ${ }^{233-236}$ The patients had a median FMA-UE score of 41.60 (Interquartile Range [IQR], 26.5-48.6) and $49.5 \%$ of them had a paresis of the right body side. 


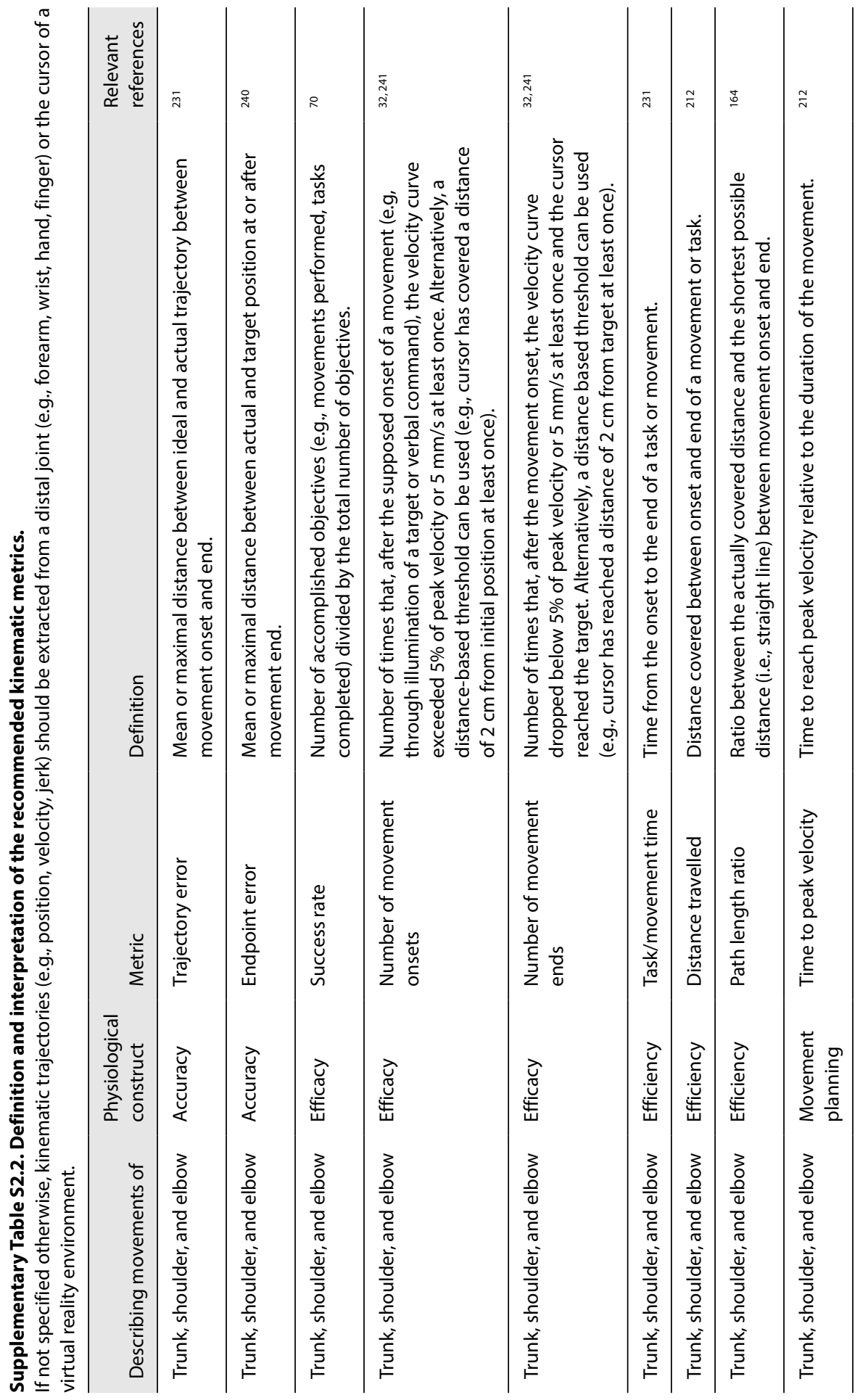




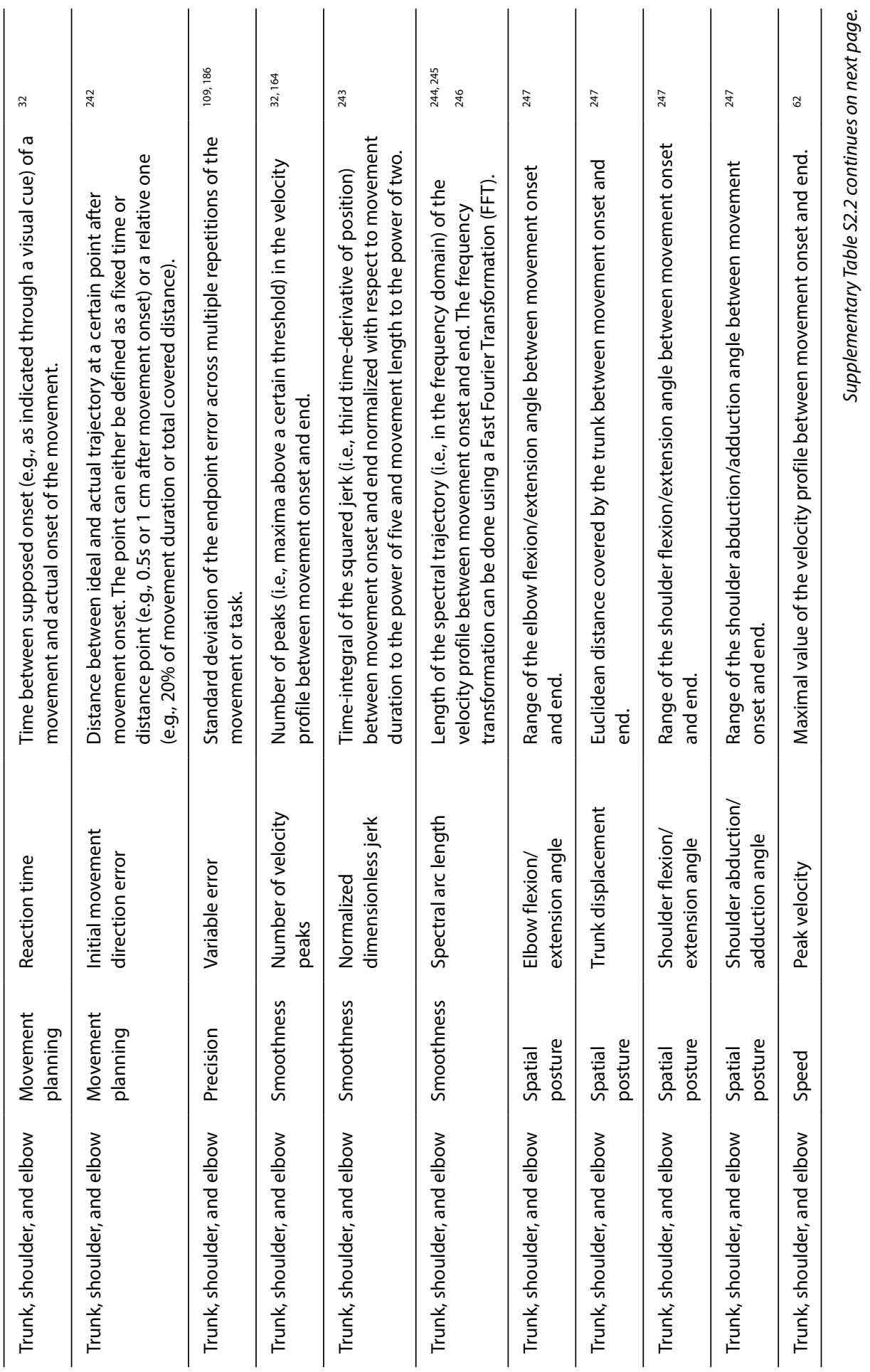




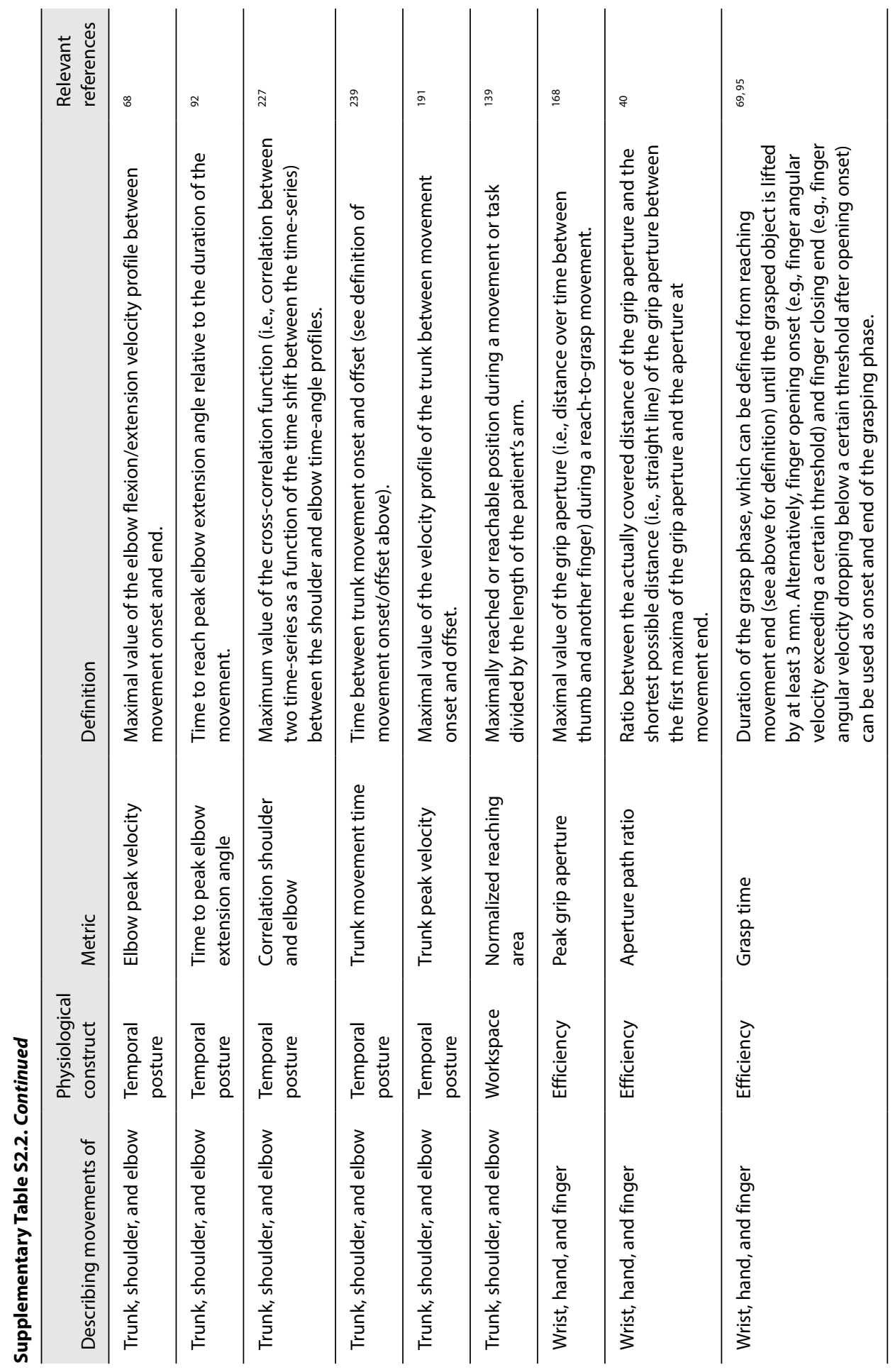




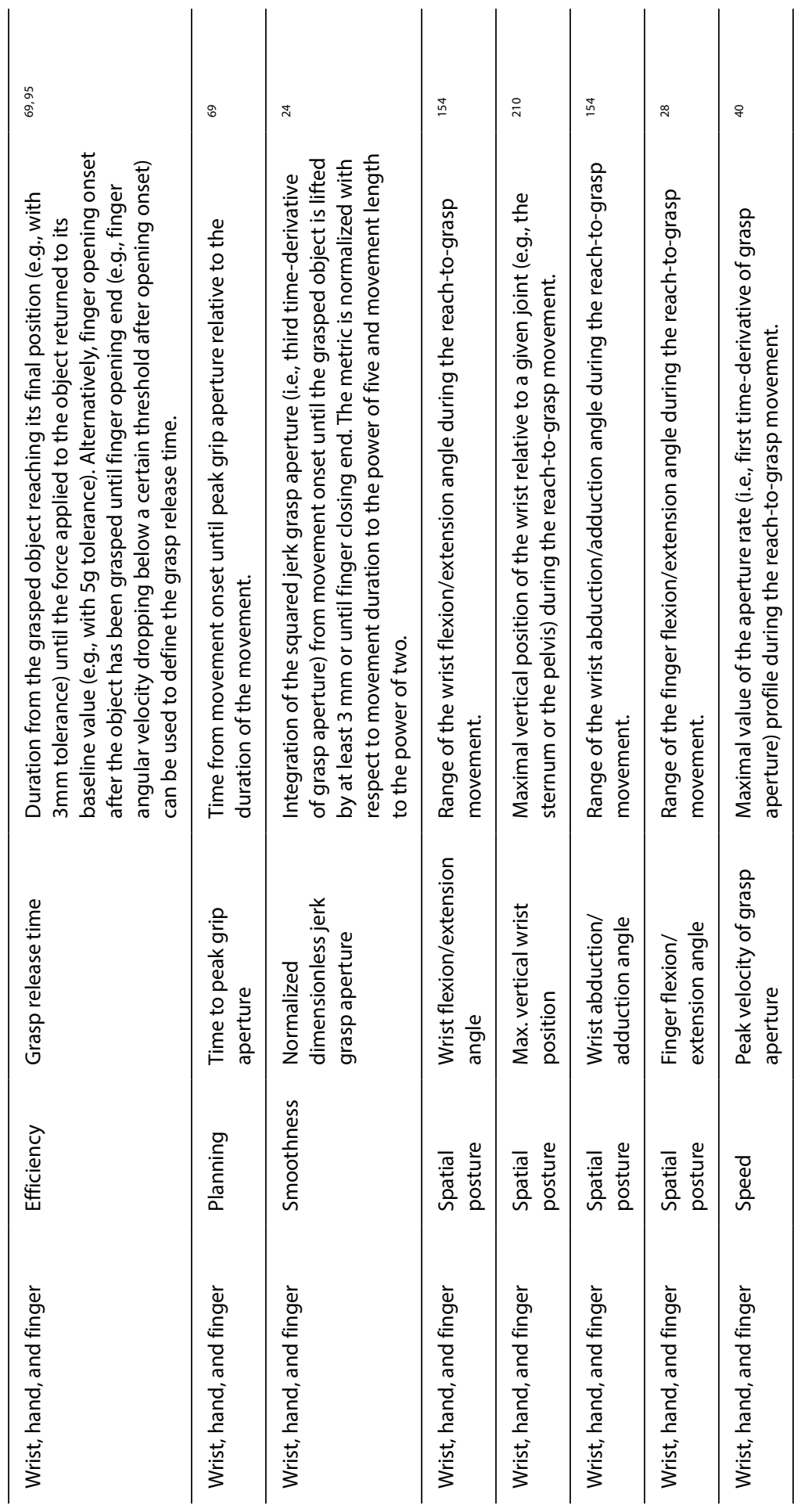




\section{2b. Kinematic assessment characteristics}

\section{TASK GROUP OVERVIEW OF UPPER LIMB KINEMATIC ASSESSMENTS POSTSTROKE}

\begin{tabular}{|c|c|}
\hline \multicolumn{2}{|c|}{ Two-dimensional tasks } \\
\hline $2 D$ pointing tasks & $\begin{array}{c}\text { 2D shape drawing } \\
\text { tasks }\end{array}$ \\
\hline $\mathrm{n}=81$ & $\mathrm{n}=16$ \\
\hline Measurement System & Measurement System \\
\hline Group A: $\quad n=20$ & Group A: $\quad n=1$ \\
\hline Group B: $\quad n=53$ & Group B: $\quad n=14$ \\
\hline Group C: $\quad n=8$ & Group C: $\quad n=1$ \\
\hline $\begin{array}{l}\text { Motor impairment } \\
\text { FMA-UE: } \\
34.35(22.40-47.59) \\
\text { (reported in } n=58 \text { ) }\end{array}$ & $\begin{array}{l}\text { Motor impairment } \\
\text { FMA-UE: } \\
33.40(22.00-45.69) \\
\text { (reported in } n=13 \text { ) }\end{array}$ \\
\hline
\end{tabular}

\begin{tabular}{|c|c|}
\hline \multicolumn{2}{|c|}{ Three-dimensional tasks } \\
\hline $3 D$ pointing tasks & $\begin{array}{c}3 D \text { reach-to-grasp } \\
\text { tasks }\end{array}$ \\
\hline $\mathrm{n}=67$ & $n=50$ \\
\hline Measurement System & Measurement System \\
\hline Group A: $\quad n=59$ & Group $\mathrm{A}: \quad \mathrm{n}=44$ \\
\hline Group B: $\quad n=4$ & Group B: $\quad n=4$ \\
\hline Group C: $\quad n=4$ & Group C: $\quad n=2$ \\
\hline $\begin{array}{l}\text { Motor impairment } \\
\text { FMA-UE: } \\
43.53(37.38-48.35 \text { ) } \\
\text { (reported in } n=48 \text { ) }\end{array}$ & $\begin{array}{l}\text { Motor impairment } \\
\text { FMA-UE: } \\
46.00(37.40-52.35 \text { ) } \\
\text { (reported in } n=32 \text { ) }\end{array}$ \\
\hline
\end{tabular}

Other tasks

$\mathrm{n}=24$
Measurement System
Group A: $\mathrm{n}=11$
Group B: $\mathrm{n}=2$
Group C: $\mathrm{n}=11$
Motorimpairment
FMA-UE:
27.35(24.4-39.23)
(reported in $\mathrm{n}=6$ )

\section{Supplementary Figure S2.1. Task overview.}

FMA-UE, Fugl-Meyer Assessment of the Upper Extremity (median [interquartile range]); Group A, systems with no to minimal influence; Group B, systems with medium influence; Group C, systems with high influence.

\section{D pointing tasks}

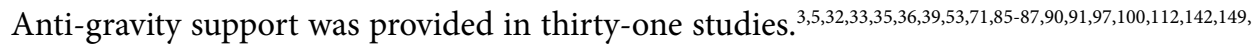
157-159,164,181,186,188,189,201,230,237 Reaching targets were arranged in center-out/star shape, ${ }^{5,35}$ spherically shape ${ }^{206}$ or in a squared shape ${ }^{4,88}$ with the number of targets ranging from three

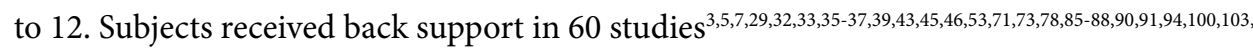
107,109,112-114,119,123,129,131,139,141,142,146, 148, 149,152,156-159,164,166,174,181,186-189,201,202,205,206,237,238 and trunk restraint in 41 studies. ${ }^{3,5,29,32,33,37,43,45,46,53,71,78,85-88,90,91,94,100,103,107,112,113,123,129,131,139,142,148,149,156-159,166,174,187,188,201,205,237}$ Two studies investigated the reliability and measurement error of the reported metrics, ${ }^{4,109}$ seven their validity, ${ }^{22,109,123,139,164,232,239}$ and one their responsiveness. ${ }^{181}$

\section{D shape drawing tasks}

Anti-gravity support was provided in five studies, ${ }^{35,101,102,118,230}$ back support in 12 studies, ${ }^{35}$, $37,52,73,98,101,102,109,116,118,132,206$ and trunk restraint in six studies. ${ }^{37,98,101,102,118,130}$ The number of task repetitions varied from three101 to $20 .{ }^{73}$ Two studies investigated the reliability and measurement error of the reported metrics ${ }^{4,109}$ and one their validity. ${ }^{22}$

\section{D pointing tasks}

Anti-gravity support was provided in six studies. ${ }^{27,42,115,120,143,172}$ Subjects had back support in 49

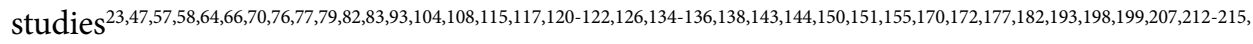
$218,219,222,224,227,229,234$ and trunk restraint in 24 studies. $23,57,58,79,83,108,120,122,126,138,150,155,172,177,182,193$, 212-214,218,219,222,227,234 Studies reported target distances from $65 \%{ }^{177}$ to $90 \%$ of subject's arm 
length. ${ }^{58,168,177}$ The number of repetitions ranged from three to 36 trials per target. Five studies investigated the reliability of the reported metrics, ${ }^{151,163,168,204,212}$ three their measurement error, ${ }^{168,204,212}$ and twelve their validity. ${ }^{41,42,64,70,104,135,145,147,151,164,204,239}$

\section{D Reach-to-grasp tasks}

Movement tasks included reach-to-grasp objects, such as a can, a ball, a cup, or a drain plunger; reach-to-grasp and open a jar, open a box and take out an object; or drinking from a cup. Subjects received anti-gravity support in two studies ${ }^{110,228}$ back support in 35 studies $^{20,21,23,25,31,34,40,41,48,51,55,59,64,68,81,92,95,96,110,111,125,137,154,160,163,185,209,211,219-221,223,228}$ and were trunk restraint in 10 studies. ${ }^{23,40,48,55,81,111,125,219-221}$ Two to nine objects were used. Placement of the targets varied between $60 \%$ and $125 \%$ of the arm's length. ${ }^{25,40,72,92,95,154,168,223}$ Trial repetitions ranged from three to 36 . One study investigated the reliability and measurement error of the reported metrics, ${ }^{168}$ six their validity, ${ }^{41,64,95,154,179,210}$ and one their responsiveness. ${ }^{25}$

\section{Other tasks}

Assessment movement tasks not fitting into one of the previous groups involved specific active joint motions, such as elbow flexion/extension, pronation/supination, and active shoulder motions; tapping and hammering tasks; gamifications such as piano games; and bimanual coordination tasks. Subjects received anti-gravity support in six studies ${ }^{49,124,153,161,167,173}$ back support in 16 studies $^{28,38,49,50,54,62,74,80,124,161,167,175,183,184,196,235}$ and were trunk restraint in four studies..$^{50,62,80,167}$ One study investigated the validity ${ }^{62}$ and one the responsiveness ${ }^{180}$ of the reported metrics.

\section{2c. Risk of bias assessment}

\section{Test-retest reliability}

Two out of eight studies (25\%) investigating test-retest reliability were of doubtful quality ${ }^{167,212}$ and six studies (75\%) were of inadequate quality. ${ }^{4,109,151,163,168,204}$ The item regarding the stability of participants in the period between tests was rated as very good or adequate in five studies. ${ }^{151,167,168,204,212}$ The time interval between tests was inadequate in six (50\%) studies. $^{4,109,151,163,168,204}$ 


\section{Measurement error}

Measurement error was investigated in six studies, out of which two (33\%) were of doubtful ${ }^{167,212}$ and four (67\%) of inadequate quality. ${ }^{4,109,168,204}$ In three studies, the time interval between tests was inadequate. ${ }^{109,168,204}$ All studies calculated the Standard Error of Measurement (SEM), Smallest Detectable Change (SDC), or Limits of Agreement (LoA).

Supplementary Table S2.3. Risk of bias for the investigated clinimetric properties for each study, according to the COSMIN checklist $(\mathbf{n}=\mathbf{3 0})$

\begin{tabular}{|c|c|c|c|c|}
\hline \multirow[b]{2}{*}{ Study } & \multicolumn{4}{|c|}{ Measurement properties } \\
\hline & Reliability & $\begin{array}{l}\text { Measurement } \\
\text { error }\end{array}$ & $\begin{array}{l}\text { Hypothesis testing for } \\
\text { construct validity * }\end{array}$ & Responsiveness ** \\
\hline Alt Murphy 2013 & & & & Doubtful \\
\hline Baniña 2017 & & & Doubtful & \\
\hline Colombo 2014 & Inadequate & Inadequate & & \\
\hline DeJong 2012a & & & Adequate & \\
\hline Finley 2012 & & & Adequate & \\
\hline Gilliaux 2014 & Inadequate & Inadequate & Doubtful & \\
\hline Kantak 2016 & & & Inadequate & \\
\hline Krebs 2014 & & & Doubtful & \\
\hline Lang 2006a & & & Adequate & \\
\hline Li 2015 & & & Inadequate & \\
\hline Longhi 2016 & & & Adequate & \\
\hline Mace 2017 & & & Very good & \\
\hline Massie 2011 & & & Doubtful & \\
\hline Massie 2014 & & & Inadequate & \\
\hline McCrea 2005 & Inadequate & & Inadequate & \\
\hline McKenzie 2017 & & & Doubtful & \\
\hline Michaelsen 2004 & & & Inadequate & \\
\hline Osu 2011 & Inadequate & & & \\
\hline Otaka 2015 & & & Very good & \\
\hline Patten 2003 & Doubtful & Doubtful & & \\
\hline Patterson 2011 & Inadequate & Inadequate & & \\
\hline Rohafza 2012 & & & Inadequate & \\
\hline Rohafza 2014 & & & & Inadequate \\
\hline Rohrer 2002 & & & & Inadequate \\
\hline Subramanian 2010 & & & Doubtful & \\
\hline Tobler-Ammann 2016 & Inadequate & Inadequate & Inadequate & \\
\hline Van Meulen 2015 & & & Inadequate & \\
\hline Wagner 2008 & Doubtful & Doubtful & & \\
\hline Wu 2014 & & & Doubtful & \\
\hline Zollo $2011 \mathrm{~b}$ & & & Inadequate & \\
\hline
\end{tabular}

* COSMIN item $9 \mathrm{~b}$ - comparison with other outcome measurements; ${ }^{* *}$, COSMIN item $10 \mathrm{~b}$ - construct approach with other outcome instruments). 


\section{Hypothesis testing}

Hypothesis testing by comparing kinematic metrics with outcome measurement instruments such as the FMA-UE and Action Research Arm Test (ARAT) was done in 22 studies. Two (9\%) were rated as very good, ${ }^{139,164}$ four (18\%) as adequate, ${ }^{41,42,95,104}$ seven $(32 \%)$ as doubtful ${ }^{22,62,64,70,109,145,239}$ and nine (41\%) as inadequate. ${ }^{122,135,147,151,154,179,204,210,232}$ Twelve studies clearly described the comparator outcome measurement instruments $41,42,62,64,70,95,109,145,154,164$, ${ }^{179,239}$ and applied appropriate statistics (i.e., correlation analysis). ${ }^{62,70,109,122,139,145,147,154,164,204,232,239}$

\section{Responsiveness}

Three studies determined responsiveness (construct approach). One study was rated as doubtful ${ }^{25}$ and two as inadequate. ${ }^{180,181}$ 


\subsection{References supplementary data}

1. Sacco RL, Kasner SE, Broderick JP, et al. An updated definition of stroke for the 21st century: A statement for healthcare professionals from the american heart association/american stroke association. Stroke. 2013;44:2064-89.

2. Bernhardt J, Hayward KS, Kwakkel G, et al. Agreed definitions and a shared vision for new standards in stroke recovery research: The stroke recovery and rehabilitation roundtable taskforce. Int J Stroke. 2017;12:444-50.

3. Colombo R, Sterpi I, Mazzone A, Pisano F, Delconte C. Modeling upper limb clinical scales by robot-measured performance parameters. IEEE Int Conf Rehabil Robot. 2011;2011:5975401.

4. Colombo R, Cusmano I, Sterpi I, Mazzone A, Delconte C, Pisano F. Test-retest reliability of robotic assessment measures for the evaluation of upper limb recovery. IEEE Trans Neural Syst Rehabil Eng. 2014;22:1020-9.

5. Goldsmith J, Kitago T. Assessing systematic effects of stroke on motorcontrol by using hierarchical function-on-scalar regression. J R Stat Soc Ser C Appl Stat. 2016;65:215-36.

6. Mottet D, van Dokkum LE, Froger J, Gouaich A, Laffont I. Trajectory formation principles are the same after mild or moderate stroke. PLoS One. 2017;12:e0173674.

7. Pila O, Duret C, Laborne FX, Gracies JM, Bayle N, Hutin E. Pattern of improvement in upper limb pointing task kinematics after a 3-month training program with robotic assistance in stroke. J Neuroeng Rehabil. 2017;14.

8. Mokkink LB, de Vet HCW, Prinsen CAC, et al. Cosmin risk of bias checklist for systematic reviews of patient-reported outcome measures. Qual Life Res. 2018;27:1171-9.

9. Terwee CB, Prinsen CAC, Chiarotto A, et al. Cosmin methodology for evaluating the content validity of patient-reported outcome measures: A delphi study. Qual Life Res. 2018;27:1159-70.

10. Bensmail D, Robertson JVG, Fermanian C, Roby-Brami A. Botulinum toxin to treat upper-limb spasticity in hemiparetic patients: Analysis of function and kinematics of reaching movements. Neurorehabil Neural Repair. 2010b;24:273-81.

11. Conrad MO, Scheidt RA, Schmit BD. Effects of wrist tendon vibration on arm tracking in people poststroke. J Neurophysiol. 2011b;106:1480-8.

12. Conrad MO, Scheidt RA, Schmit BD. Effects of wrist tendon vibration on targeted upper-arm movements in poststroke hemiparesis. Neurorehabil Neural Repair. 2011c;25:61-70.

13. Krabben T, Prange GB, Molier BI, Rietman JS, Buurke JH. Objective measurement of synergistic movement patterns of the upper extremity following stroke: An explorative study. IEEE Int Con Rehabil Robot. 2011b;2011:5975430.

14. Mirbagheri MM, Tsao CC, Rymer WZ. Changes of elbow kinematics and kinetics during 1 year after stroke. Muscle Nerve. 2008b;37:387-95.

15. Park H, Kim S, Winstein CJ, Gordon J, Schweighofer N. Short-duration and intensive training improves long-term reaching performance in individuals with chronic stroke. Neurorehabil Neural Repair. 2016;30:551-61.

16. Robertson JV, Roby-Brami A. The trunk as a part of the kinematic chain for reaching movements in healthy subjects and hemiparetic patients. Brain Res. 2011;1382:137-46.

17. Simkins M, Jacobs AB, Byl N, Rosen J. Stroke-induced synergistic phase shifting and its possible implications for recovery mechanisms. Exp Brain Res. 2014b;232:3489-99. 
18. Stewart JC, Gordon J, Winstein CJ. Control of reach extent with the paretic and nonparetic arms after unilateral sensorimotor stroke: Kinematic differences based on side of brain damage. Exp Brain Res. 2014b;232:2407-19.

19. Yu L, Wang Jp, Fang Q, Wang Y. Brunnstrom stage automatic evaluation for stroke patients using extreme learning machine. IEEE Biomed Circuit Syst Conf. 2012:380-3.

20. Alt Murphy M, Willen C, Sunnerhagen KS. Movement kinematics during a drinking task are associated with the activity capacity level after stroke. Neurorehabil Neural Repair. 2012;26:110615.

21. Edwards DF, Lang CE, Wagner JM, Birkenmeier R, Dromerick AW. An evaluation of the wolf motor function test in motor trials early after stroke. Arch Phys Med Rehabil. 2012;93:660-8.

22. Krebs HI, Krams M, Agrafiotis DK, et al. Robotic measurement of arm movements after stroke establishes biomarkers of motor recovery. Stroke. 2014;45:200-4.

23. Lang CE, Wagner JM, Edwards DF, Sahrmann SA, Dromerick AW. Recovery of grasp versus reach in people with hemiparesis poststroke. Neurorehabil Neural Repair. 2006b;20:444-54.

24. van Kordelaar J, van Wegen E, Kwakkel G. Impact of time on quality of motor control of the paretic upper limb after stroke. Arch Phys Med Rehabil. 2014;95:338-44.

25. Alt Murphy M, Willen C, Sunnerhagen KS. Responsiveness of upper extremity kinematic measures and clinical improvement during the first three months after stroke. Neurorehabil Neural Repair. 2013;27:844-53.

26. Altenmuller E, Marco-Pallares J, Munte TF, Schneider S. Neural reorganization underlies improvement in stroke-induced motor dysfunction by music-supported therapy. Ann. N.Y. Acad. 2009;1169:395-405.

27. Bartolo M, De Nunzio AM, Sebastiano F, et al. Arm weight support training improves functional motor outcome and movement smoothness after stroke. Funct Neurol. 2014;29:15-21.

28. Beebe JA, Lang CE. Active range of motion predicts upper extremity function 3 months after stroke. Stroke. 2009;40:1772-9.

29. Bourke TC, Coderre AM, Bagg SD, Dukelow SP, Norman KE, Scott SH. Impaired corrective responses to postural perturbations of the arm in individuals with subacute stroke. J Neuroeng Rehabil. 2015;12:7.

30. Buma FE, van Kordelaar J, Raemaekers M, van Wegen EE, Ramsey NF, Kwakkel G. Brain activation is related to smoothness of upper limb movements after stroke. Exp Brain Res. 2016;234:2077-89.

31. Bustren EL, Sunnerhagen KS, Alt Murphy M. Movement kinematics of the ipsilesional upper extremity in persons with moderate or mild stroke. Neurorehabil Neural Repair. 2017;31:376-86.

32. Coderre AM, Zeid AA, Dukelow SP, et al. Assessment of upper-limb sensorimotor function of subacute stroke patients using visually guided reaching. Neurorehabil Neural Repair. 2010;24:52841.

33. Cortes JC, Goldsmith J, Harran MD, et al. A short and distinct time window for recovery of arm motor control early after stroke revealed with a global measure of trajectory kinematics. Neurorehabil Neural Repair. 2017;31:552-60.

34. DeJong SL, Schaefer SY, Lang CE. Need for speed: Better movement quality during faster task performance after stroke. Neurorehabil Neural Repair. 2012c;26:362-73.

35. Dipietro L, Krebs HI, Volpe BT, et al. Learning, not adaptation, characterizes stroke motor recovery: Evidence from kinematic changes induced by robot-assisted therapy in trained and untrained task in the same workspace. IEEE Trans Neural Syst Rehabil Eng. 2012;20:48-57. 
36. Dukelow SP, Herter TM, Bagg SD, Scott SH. The independence of deficits in position sense and visually guided reaching following stroke. J Neuroeng Rehabil. 2012;9:72.

37. Gilliaux M, Lejeune T, Detrembleur C, Sapin J, Dehez B, Stoquart G. A robotic device as a sensitive quantitative tool to assess upper limb impairments in stroke patients: A preliminary prospective cohort study. J Rehabil Med. 2012;44:210-7.

38. Kim WS, Cho S, Baek D, Bang H, Paik NJ. Upper extremity functional evaluation by fugl-meyer assessment scoring using depth-sensing camera in hemiplegic stroke patients. PLoS ONE. 2016;11.

39. Krebs HI, Aisen ML, Volpe BT, Hogan N. Quantization of continuous arm movements in humans with brain injury. Proc Natl Acad Sciences U S A. 1999;96:4645-9.

40. Lang CE, Wagner JM, Bastian AJ, et al. Deficits in grasp versus reach during acute hemiparesis. Exp Brain Res. 2005;166:126-36.

41. Lang CE, Wagner JM, Dromerick AW, Edwards DF. Measurement of upper-extremity function early after stroke: Properties of the action research arm test. Arch Phys Med Rehabil. 2006a; 87:1605-10.

42. Longhi M, Merlo A, Prati P, Giacobbi M, Mazzoli D. Instrumental indices for upper limb function assessment in stroke patients: A validation study. J Neuroeng Rehabil. 2016;13:52.

43. Mazzoleni S, Filippi M, Carrozza MC, Posteraro F, Puzzolante L, Falchi E. Robot-aided therapy on the upper limb of subacute and chronic stroke patients: A biomechanical approach. IEEE Int Con Rehabil Robot. 2011;2011:5975422.

44. Mazzoleni S, Carrozza MC, Sale P, Franceschini M, Posteraro F, Tiboni M. Effects of upper limb robot-assisted therapy on motor recovery of subacute stroke patients: A kinematic approach. IEEE Int Con Rehabil Robot. 2013a;2013:6650503.

45. Mazzoleni S, Sale P, Tiboni M, Franceschini M, Carrozza MC, Posteraro F. Upper limb robotassisted therapy in chronic and subacute stroke patients. Am J Phys Med Rehabil. 2013c:e26-37.

46. Mazzoleni S, Buono L, Dario P, Posteraro F. Upper limb robot-assisted therapy in subacute and chronic stroke patients: Preliminary results on initial exposure based on kinematic measures. IEEE RAS/EMBS Int Con Biomed Robot Biomechatron. 2014a:265-9.

47. Messier S, Bourbonnais D, Desrosiers J, Roy Y. Kinematic analysis of upper limbs and trunk movement during bilateral movement after stroke. Arch Phys Med Rehabil. 2006;87:1463-70.

48. Metrot J, Mottet D, Hauret I, et al. Changes in bimanual coordination during the first 6 weeks after moderate hemiparetic stroke. Neurorehabil Neural Repair. 2013;27:251-9.

49. Mirbagheri MM, Rymer WZ. Predication of motor recovery using kinematic and kinetic measures. 5th Int IEEE/EMBS Con Neural Eng. 2011:617-20.

50. Mirbagheri MM, Rymer WZ. Time-course of changes in arm impairment after stroke: Variables predicting motor recovery over 12 months. Arch Phys Med Rehabil. 2008a;89:1507-13

51. Nowak DA, Grefkes C, Dafotakis M, et al. Effects of low-frequency repetitive transcranial magnetic stimulation of the contralesional primary motor cortex on movement kinematics and neural activity in subcortical stroke. Arch Neurol. 2008;65:741-7.

52. Sakurada T, Nakajima T, Morita M, Hirai M, Watanabe E. Improved motor performance in patients with acute stroke using the optimal individual attentional strategy. Sci Rep. 2017;7:40592.

53. Semrau JA, Herter TM, Kenzie JM, Findlater SE, Scott SH, Dukelow SP. Robotic characterization of ipsilesional motor function in subacute stroke. Neurorehabil Neural Repair. 2017;31:571-82. 
54. Van Delden AEQ, Beek PJ, Roerdink M, Kwakkel G, Peper CE. Unilateral and bilateral upperlimb training interventions after stroke have similar effects on bimanual coupling strength. Neurorehabil Neural Repair. 2015;29:255-67.

55. van Dokkum L, Hauret I, Mottet D, Froger J, Metrot J, Laffont I. The contribution of kinematics in the assessment of upper limb motor recovery early after stroke. Neurorehabil Neural Repair. 2014;28:4-12.

56. van Kordelaar J, van Wegen EE, Nijland RH, Daffertshofer A, Kwakkel G. Understanding adaptive motor control of the paretic upper limb early poststroke: The explicit-stroke program. Neurorehabil Neural Repair. 2013;27:854-63.

57. Wagner JM, Lang CE, Sahrmann SA, Edwards DF, Dromerick AW. Sensorimotor impairments and reaching performance in subjects with poststroke hemiparesis during the first few months of recovery. Phys Ther. 2007;87:751-65.

58. Wagner JM, Lang CE, Sahrmann SA, et al. Relationships between sensorimotor impairments and reaching deficits in acute hemiparesis. Neurorehabil Neural Repair. 2006;20:406-16.

59. Bang DH, Shin WS, Choi SJ. The effects of modified constraint-induced movement therapy combined with trunk restraint in subacute stroke: A double-blinded randomized controlled trial. Clin Rehabil. 2015;29:561-9.

60. De Baets L, Van Deun S, Monari D, Jaspers E. Three-dimensional kinematics of the scapula and trunk, and associated scapular muscle timing in individuals with stroke. Hum Mov Sci. 2016;48:82-90.

61. Kiper P, Agostini M, Luque-Moreno C, Tonin P, Turolla A. Reinforced feedback in virtual environment for rehabilitation of upper extremity dysfunction after stroke: Preliminary data from a randomized controlled trial. BioMed Res Int. 2014;2014:752128.

62. McKenzie A, Dodakian L, See J, et al. Validity of robot-based assessments of upper extremity function. Arch Phys Med Rehabil. 2017;98:1969-1976.e1962.

63. Robertson JV, Hoellinger T, Lindberg P, Bensmail D, Hanneton S, Roby-Brami A. Effect of auditory feedback differs according to side of hemiparesis: A comparative pilot study. J Neuroeng Rehabil. 2009;6:45.

64. Subramanian SK, Yamanaka J, Chilingaryan G, Levin MF. Validity of movement pattern kinematics as measures of arm motor impairment poststroke. Stroke. 2010;41:2303-8.

65. van Vliet PM, Sheridan MR. Coordination between reaching and grasping in patients with hemiparesis and healthy subjects. Arch Phys Med Rehabil. 2007;88:1325-31.

66. Adams RJ, Lichter MD, Krepkovich ET, Ellington A, White M, Diamond PT. Assessing upper extremity motor function in practice of virtual activities of daily living. IEEE Trans Neural Syst Rehabil Eng. 2015;23:287-96.

67. Alt Murphy M, Baniña MC, Levin MF. Perceptuo-motor planning during functional reaching after stroke. Exp Brain Res. 2017;235:3295-306.

68. Alt Murphy M, Willen C, Sunnerhagen KS. Kinematic variables quantifying upper-extremity performance after stroke during reaching and drinking from a glass. Neurorehabil Neural Repair. 2011;25:71-80.

69. Baak B, Bock O, Dovern A, Saliger J, Karbe H, Weiss PH. Deficits of reach-to-grasp coordination following stroke: Comparison of instructed and natural movements. Neuropsychologia. 2015;77:1-9. 
70. Baniña MC, Mullick AA, McFadyen BJ, Levin MF. Upper limb obstacle avoidance behavior in individuals with stroke. Neurorehabil Neural Repair. 2017;31:133-46.

71. Beer RF, Ellis MD, Holubar BG, Dewald JP. Impact of gravity loading on post-stroke reaching and its relationship to weakness. Muscle Nerve. 2007;36:242-50.

72. Bensmail D, Robertson J, Fermanian C, Roby-Brami A. Botulinum toxin to treat upper-limb spasticity in hemiparetic patients: Grasp strategies and kinematics of reach-to-grasp movements. Neurorehabil Neural Repair. 2010a;24:141-51.

73. Bosecker C, Dipietro L, Volpe B, Krebs HI. Kinematic robot-based evaluation scales and clinical counterparts to measure upper limb motor performance in patients with chronic stroke. Neurorehabil Neural Repair. 2010;24:62-9.

74. Bouchard AE, Corriveau H, Milot MH. A single robotic session that guides or increases movement error in survivors post-chronic stroke: Which intervention is best to boost the learning of a timing task? Disabil Rehabil. 2017;39:1607-14.

75. Brodie SM, Meehan S, Borich MR, Boyd LA. 5 hz repetitive transcranial magnetic stimulation over the ipsilesional sensory cortex enhances motor learning after stroke. Front Hum Neurosci. 2014;8:143.

76. Brokaw EB, Holley RJ, Lum PS. Comparison of joint space and end point space robotic training modalities for rehabilitation of interjoint coordination in individuals with moderate to severe impairment from chronic stroke. IEEE Trans Neural Syst Rehabil Eng 2013;21:787-95.

77. Cameirao MS, Badia SB, Oller ED, Verschure PF. Neurorehabilitation using the virtual reality based rehabilitation gaming system: Methodology, design, psychometrics, usability and validation. J Neuroeng Rehabil. 2010;7:48.

78. Casadio M, Giannoni P, Morasso P, Sanguineti V. A proof of concept study for the integration of robot therapy with physiotherapy in the treatment of stroke patients. Clin Rehabil. 2009;23:21728.

79. Chang JJ, Tung WL, Wu WL, Huang MH, Su FC. Effects of robot-aided bilateral force-induced isokinetic arm training combined with conventional rehabilitation on arm motor function in patients with chronic stroke. Arch Phys Med Rehabil. 2007;88:1332-8.

80. Chang JJ, Yang YS, Guo LY, Wu WL, Su FC. Differences in reaching performance between normal adults and patients post stroke-a kinematic analysis. J Med Biol Eng. 2008a;28:53-8.

81. Chang J-J, Yang Y-S, Wu W-L, Guo L-Y, Su F-C. The constructs of kinematic measures for reaching performance in stroke patients. J Med Biol Eng. 2008b;28:65-70.

82. Chen HL, Lin KC, Liing RJ, Wu CY, Chen CL. Kinematic measures of arm-trunk movements during unilateral and bilateral reaching predict clinically important change in perceived arm use in daily activities after intensive stroke rehabilitation. J Neuroeng Rehabil. 2015;12:84.

83. Cho KH, Song WK. Robot-assisted reach training for improving upper extremity function of chronic stroke. Tohoku J Exp Med. 2015;237:149-55.

84. Cirstea MC, Levin MF. Improvement of arm movement patterns and endpoint control depends on type of feedback during practice in stroke survivors. Neurorehabil Neural Repair. 2007;21:398411.

85. Colombo R, Pisano F, Micera S, et al. Robotic techniques for upper limb evaluation and rehabilitation of stroke patients. IEEE Trans Neural Syst Rehabil Eng. 2005;13:311-24.

86. Colombo R, Pisano F, Micera S, et al. Assessing mechanisms of recovery during robot-aided neurorehabilitation of the upper limb. Neurorehabil Neural Repair. 2008;22:50-63. 
87. Colombo R, Sterpi I, Mazzone A, Delconte C, Minuco G, Pisano F. Measuring changes of movement dynamics during robot-aided neurorehabilitation of stroke patients. IEEE Neural Syst Rehabil Eng. 2010;18:75-85.

88. Colombo R, Sterpi I, Mazzone A, Delconte C, Pisano F. Taking a lesson from patients' recovery strategies to optimize training during robot-aided rehabilitation. IEEE Neural Syst Rehabil Eng. 2012;20:276-85.

89. Colombo R, Pisano F, Delconte C, et al. Comparison of exercise training effect with different robotic devices for upper limb rehabilitation: A retrospective study. Eur J Phys Rehabil Med. 2017;53:240-8.

90. Conrad MO, Gadhoke B, Scheidt RA, Schmit BD. Effect of tendon vibration on hemiparetic arm stability in unstable workspaces. PLoS One. 2015a;10:e0144377.

91. Conroy SS, Whitall J, Dipietro L, et al. Effect of gravity on robot-assisted motor training after chronic stroke: A randomized trial. Arch Phys Med Rehabil. 2011;92:1754-61.

92. Corti M, McGuirk TE, Wu SS, Patten C. Differential effects of power training versus functional task practice on compensation and restoration of arm function after stroke. Neurorehabil Neural Repair. 2012;26:842-54.

93. de Oliveira Cacho R, Cacho EW, Ortolan RL, Cliquet A, Jr., Borges G. Trunk restraint therapy: The continuous use of the harness could promote feedback dependence in poststroke patients: A randomized trial. Medicine. 2015;94:e641.

94. de Paiva Silva FP, Freitas SM, Silva PV, Banjai RM, Alouche SR. Ipsilesional arm motor sequence performance after right and left hemisphere damage. J Mot Behav. 2014;46:407-14.

95. DeJong SL, Birkenmeier RL, Lang CE. Person-specific changes in motor performance accompany upper extremity functional gains after stroke. J Appl Biomech. 2012a;28:304-16.

96. Dejong SL, Lang CE. Comparison of unilateral versus bilateral upper extremity task performance after stroke. Top Stroke Rehabil. 2012b;19:294-305.

97. Di Lazzaro V, Capone F, Di Pino G, et al. Combining robotic training and non-invasive brain stimulation in severe upper limb-impaired chronic stroke patients. Front Neurosci. 2016;10:88.

98. Dipietro L, Krebs HI, Fasoli SE, et al. Changing motor synergies in chronic stroke. J Neurophysiol. 2007;98:757-68.

99. Durham KF, Sackley CM, Wright CC, Wing AM, Edwards MG, van Vliet P. Attentional focus of feedback for improving performance of reach-to-grasp after stroke: A randomised crossover study. Physiotherapy. 2014;100:108-15.

100. Ellis MD, Carmona C, Drogos J, Traxel S, Dewald JP. Progressive abduction loading therapy targeting flexion synergy to regain reaching function in chronic stroke: Preliminary results from an rct. Ann Int Con IEEE Eng Med Biol Soc. 2016;2016:5837-40.

101. Ellis MD, Sukal T, DeMott T, Dewald JP. Augmenting clinical evaluation of hemiparetic arm movement with a laboratory-based quantitative measurement of kinematics as a function of limb loading. Neurorehabil Neural Repair. 2008;22:321-9.

102. Ellis MD, Sukal-Moulton T, Dewald JP. Progressive shoulder abduction loading is a crucial element of arm rehabilitation in chronic stroke. Neurorehabil Neural Repair. 2009;23:862-9.

103. Finley MA, Fasoli SE, Dipietro L, et al. Short-duration robotic therapy in stroke patients with severe upper-limb motor impairment. J Rehabil Res Dev. 2005;42:683-92. 
104. Finley M, Combs S, Carnahan K, Peacock S, Buskirk AV. Comparison of 'less affected limb' reaching kinematics in individuals with chronic stroke and healthy age-matched controls. Phys Occup Ther Geriatr. 2012;30:245-59.

105. Fleming MK, Newham DJ, Rothwell JC. Explicit motor sequence learning with the paretic arm after stroke. Disabil Rehabil. 2016:1-6.

106. Fluet GG, Merians AS, Qiu Q, Rohafaza M, VanWingerden AM, Adamovich SV. Does training with traditionally presented and virtually simulated tasks elicit differing changes in object interaction kinematics in persons with upper extremity hemiparesis? Top Stroke Rehabil. 2015;22:176-84.

107. Gao KL, Ng SS, Kwok JW, Chow RT, Tsang WW. Eye-hand coordination and its relationship with sensori-motor impairments in stroke survivors. J Rehabil Med. 2010;42:368-73.

108. Gera G, Freitas SM, Scholz JP. Relationship of diminished interjoint coordination after stroke to hand path consistency. Exp Brain Res. 2016;234:741-51.

109. Gilliaux M, Lejeune TM, Detrembleur C, et al. Using the robotic device reaplan as a valid, reliable, and sensitive tool to quantify upper limb impairments in stroke patients. J Rehabil Med. 2014;46:117-25.

110. Grimm F, Gharabaghi A. Closed-loop neuroprosthesis for reach-to-grasp assistance: Combining adaptive multi-channel neuromuscular stimulation with a multi-joint arm exoskeleton. Front Neurosci. 2016;10:284.

111. Guttman A, Burstin A, Brown R, Bril S, Dickstein R. Motor imagery practice for improving sit to stand and reaching to grasp in individuals with poststroke hemiparesis. Top Stroke Rehabil. 2012;19:306-19.

112. Hammerbeck U, Yousif N, Hoad D, Greenwood R, Diedrichsen J, Rothwell JC. Chronic stroke survivors improve reaching accuracy by reducing movement variability at the trained movement speed. Neurorehabil Neural Repair. 2017;31:499-508.

113. Han CE, Kim S, Chen S, et al. Quantifying arm nonuse in individuals poststroke. Neurorehabil Neural Repair. 2013;27:439-47.

114. Hardwick RM, Rajan VA, Bastian AJ, Krakauer JW, Celnik PA. Motor learning in stroke: Trained patients are not equal to untrained patients with less impairment. Neurorehabil Neural Repair. 2017;31:178-89.

115. Housman SJ, Scott KM, Reinkensmeyer DJ. A randomized controlled trial of gravity-supported, computer-enhanced arm exercise for individuals with severe hemiparesis. Neurorehabil Neural Repair. 2009;23:505-14.

116. Houwink A, Steenbergen B, Prange GB, Buurke JH, Geurts AC. Upper-limb motor control in patients after stroke: Attentional demands and the potential beneficial effects of arm support. Hum Mov Sci. 2013;32:377-87.

117. Hsieh YW, Liing RJ, Lin KC, et al. Sequencing bilateral robot-assisted arm therapy and constraintinduced therapy improves reach to press and trunk kinematics in patients with stroke. J Neuroeng Rehabil. 2016;13:31.

118. Huang FC, Patton JL. Augmented dynamics and motor exploration as training for stroke. IEEE Trans Biomed Eng. 2013;60:838-44.

119. Hussain A, Budhota A, Hughes CML, et al. Self-paced reaching after stroke: A quantitative assessment of longitudinal and directional sensitivity using the h-man planar robot for upper limb neurorehabilitation. Front Neurosci. 2016;10. 
120. Iwamuro BT, Cruz EG, Connelly LL, Fischer HC, Kamper DG. Effect of a gravity-compensating orthosis on reaching after stroke: Evaluation of the therapy assistant wrex. Arch Phys Med Rehabil. 2008;89:2121-8.

121. Johansson GM, Grip H, Levin MF, Hager CK. The added value of kinematic evaluation of the timed finger-to-nose test in persons post-stroke. J Neuroeng Rehabil. 2017;14:11.

122. Kantak SS, Zahedi N, McGrath RL. Task-dependent bimanual coordination after stroke: Relationship with sensorimotor impairments. Arch Phys Med Rehabil. 2016;97:798-806.

123. Kantak SS, Zahedi N, McGrath R. Complex skill training transfers to improved performance and control of simpler tasks after stroke. Phys Ther. 2017;97:718-28.

124. Kim H, Miller LM, Fedulow I, et al. Kinematic data analysis for post-stroke patients following bilateral versus unilateral rehabilitation with an upper limb wearable robotic system. IEEE Trans Neural Syst Rehabil Eng. 2013;21:153-64.

125. Kim H, Yoo EY, Jung MY, Kim J, Park JH, Kang DH. The effects of mental practice combined with modified constraint-induced therapy on corticospinal excitability, movement quality, function, and activities of daily living in persons with stroke. Disabil Rehabil. 2017:1-9.

126. Kim JR, Jung MY, Yoo EY, Park JH, Kim SH, Lee J. Effects of rhythmic auditory stimulation during hemiplegic arm reaching in individuals with stroke: An exploratory study. Hong Kong J Occup Ther. 2014a;24:64-71.

127. Kim K, Song WK, Lee J, et al. Kinematic analysis of upper extremity movement during drinking in hemiplegic subjects. Clin Biomech. 2014b;29:248-56.

128. Koesler IB, Dafotakis M, Ameli M, Fink GR, Nowak DA. Electrical somatosensory stimulation improves movement kinematics of the affected hand following stroke. J Neurol Neurosurg Psychiatry. 2009;80:614-9.

129. Kostic M, Popovic MB, Popovic DB. A method for assessing the arm movement performance: Probability tube. Med Biol Eng Comput. 2013;51:1315-23.

130. Krabben T, Molier BI, Houwink A, Rietman JS, Buurke JH, Prange GB. Circle drawing as evaluative movement task in stroke rehabilitation: An explorative study. J Neuroeng Rehabil. 2011a;8:15.

131. Leconte P, Orban de Xivry JJ, Stoquart G, Lejeune T, Ronsse R. Rhythmic arm movements are less affected than discrete ones after a stroke. Exp Brain Res. 2016;234:1403-17.

132. Lefebvre S, Laloux P, Peeters A, Desfontaines P, Jamart J, Vandermeeren Y. Dual-tdcs enhances online motor skill learning and long-term retention in chronic stroke patients. Front Human Neurosci. 2012;6:343.

133. Levin MF, Magdalon EC, Michaelsen SM, Quevedo AA. Quality of grasping and the role of haptics in a 3-d immersive virtual reality environment in individuals with stroke. IEEE Trans Neural Syst Rehabil Eng. 2015;23:1047-55.

134. Levin MF, Liebermann DG, Parmet Y, Berman S. Compensatory versus noncompensatory shoulder movements used for reaching in stroke. Neurorehabil Neural Repair. 2016;30:635-46.

135. Li KY, Lin KC, Chen CK, Liing RJ, Wu CY, Chang WY. Concurrent and predictive validity of arm kinematics with and without a trunk restraint during a reaching task in individuals with stroke. Arch Phys Med Rehabil. 2015;96:1666-75.

136. Liebermann DG, Berman S, Weiss PL, Levin MF. Kinematics of reaching movements in a 2-d virtual environment in adults with and without stroke. IEEE Trans Neural Syst Rehabil Eng. 2012;20:778-87. 
137. Lima RCM, Michaelsen SM, Nascimento LR, Polese JC, Pereira ND, Teixeira-Salmela LF. Addition of trunk restraint to home-based modified constraint-induced movement therapy does not bring additional benefits in chronic stroke individuals with mild and moderate upper limb impairments: A pilot randomized controlled trial. NeuroRehabilitation. 2014;35:391-404.

138. Lin KC, Chen YA, Chen CL, Wu CY, Chang YF. The effects of bilateral arm training on motor control and functional performance in chronic stroke: A randomized controlled study. Neurorehabil Neural Repair. 2010;24:42-51.

139. Mace M, Guy S, Hussain A, et al. Validity of a sensor-based table-top platform to measure upper limb function. Int Con Rehabil Robot. 2017:652-7.

140. Ma HI, Lin KC, Hsieh FH, Chen CL, Tang SF, Wu CY. Kinematic manifestation of arm-trunk performance during symmetric bilateral reaching after stroke: Within vs. Beyond arm's length. Am J Phys Med Rehabil. 2017;96:146-51.

141. Mandon L, Boudarham J, Robertson J, Bensmail D, Roche N, Roby-Brami A. Faster reaching in chronic spastic stroke patients comes at the expense of arm-trunk coordination. Neurorehabil Neural Repair. 2016;30:209-20.

142. Mani S, Mutha PK, Przybyla A, Haaland KY, Good DC, Sainburg RL. Contralesional motor deficits after unilateral stroke reflect hemisphere-specific control mechanisms. Brain. 2013;136:1288-303.

143. Maris A, Coninx K, Seelen H, et al. The impact of robot-mediated adaptive i-travle training on impaired upper limb function in chronic stroke and multiple sclerosis. Disabil Rehabil Assist Technol. 2017:1-9.

144. Massie C, Malcolm MP, Greene D, Thaut M. The effects of constraint-induced therapy on kinematic outcomes and compensatory movement patterns: An exploratory study. Arch Phys Med Rehabil. 2009;90:571-9.

145. Massie CL, Fritz S, Malcolm MP. Elbow extension predicts motor impairment and performance after stroke. Rehabil Res Pract. 2011;2011:381978.

146. Massie CL, Malcolm MP, Greene DP, Browning RC. Kinematic motion analysis and muscle activation patterns of continuous reaching in survivors of stroke. J Mot Behav. 2012;44:213-22.

147. Massie C, Malcolm M, Greene D, Browning R. Biomechanical contributions of the trunk and upper extremity in discrete versus cyclic reaching in survivors of stroke. Top Stroke Rehabil. 2014;21:23-32.

148. Mazzoleni S, Crecchi R, Posteraro F, Carrozza MC. Robot-assisted upper limb rehabilitation in chronic stroke patients. Ann Int Con IEEE Eng Med Biol Soc. 2013b;2013:886-9.

149. Mazzoleni S, Puzzolante L, Zollo L, Dario P, Posteraro F. Mechanisms of motor recovery in chronic and subacute stroke patients following a robot-aided training. IEEE Trans haptics. 2014b;7:175-80.

150. McCombe Waller S, Liu W, Whitall J. Temporal and spatial control following bilateral versus unilateral training. Hum Mov Sci. 2008;27:749-58.

151. McCrea PH, Eng JJ, Hodgson AJ. Saturated muscle activation contributes to compensatory reaching strategies after stroke. J Neurophysiol. 2005;94:2999-3008.

152. Merdler T, Liebermann DG, Levin MF, Berman S. Arm-plane representation of shoulder compensation during pointing movements in patients with stroke. J Electromyogr Kinesiol. 2013;23:938-47.

153. Merians AS, Fluet GG, Qiu Q, et al. Robotically facilitated virtual rehabilitation of arm transport integrated with finger movement in persons with hemiparesis. J Neuroeng Rehabil. 2011;8:27. 
154. Michaelsen SM, Jacobs S, Roby-Brami A, Levin MF. Compensation for distal impairments of grasping in adults with hemiparesis. Exp Brain Res. 2004;157:162-73.

155. Mohapatra S, Harrington R, Chan E, Dromerick AW, Breceda EY, Harris-Love M. Role of contralesional hemisphere in paretic arm reaching in patients with severe arm paresis due to stroke: A preliminary report. Neurosci Lett. 2016;617:52-8.

156. Mukherjee M, Koutakis P, Siu KC, Fayad PB, Stergiou N. Stroke survivors control the temporal structure of variability during reaching in dynamic environments. Ann Biomed Eng. 2013;41:36676.

157. Mutha PK, Sainburg RL, Haaland KY. Coordination deficits in ideomotor apraxia during visually targeted reaching reflect impaired visuomotor transformations. Neuropsychologia. 2010;48:385567.

158. Mutha PK, Sainburg RL, Haaland KY. Critical neural substrates for correcting unexpected trajectory errors and learning from them. Brain. 2011a;134:3647-61.

159. Mutha PK, Sainburg RL, Haaland KY. Left parietal regions are critical for adaptive visuomotor control. J Neurosci. 2011b;31:6972-81.

160. Nijenhuis S, Prange G, Stienentt A, Buurke J, Rietman J. Direct effect of a dynamic wrist and hand orthosis on reach and grasp kinematics in chronic stroke. IEEE Int Con Rehabil Robot. 2015;404-9.

161. Notley SV, Turk R, Pickering R, Simpson DM, Burridge JH. Analysis of the quality of wrist movement during a simple tracking task. Physiol Meas. 2007;28:881-95.

162. Nowak DA, Grefkes C, Dafotakis M, Kust J, Karbe H, Fink GR. Dexterity is impaired at both hands following unilateral subcortical middle cerebral artery stroke. Eur J Neurosci. 2007;25:3173-84.

163. Osu R, Ota K, Fujiwara T, Otaka Y, Kawato M, Liu M. Quantifying the quality of hand movement in stroke patients through three-dimensional curvature. J Neuroeng Rehabil. 2011;8:62.

164. Otaka E, Otaka Y, Kasuga S, et al. Clinical usefulness and validity of robotic measures of reaching movement in hemiparetic stroke patients. J Neuroeng Rehabil. 2015;12:66.

165. Panarese A, Pirondini E, Tropea P, Cesqui B, Posteraro F, Micera S. Model-based variables for the kinematic assessment of upper-extremity impairments in post-stroke patients. J Neuroeng Rehabil. 2016;13:81.

166. Park H, Schweighofer N. Nonlinear mixed-effects model reveals a distinction between learning and performance in intensive reach training post-stroke. J Neuroeng Rehabil. 2017;14:21.

167. Patten C, Kothari D, Whitney J, Lexell J, Lum PS. Reliability and responsiveness of elbow trajectory tracking in chronic poststroke hemiparesis. J Rehabil Res Dev. 2003;40:487-500.

168. Patterson TS, Bishop MD, McGuirk TE, Sethi A, Richards LG. Reliability of upper extremity kinematics while performing different tasks in individuals with stroke. J Motor Behav. 2011;43:121-30.

169. Piron L, Tonin P, Cortese F, et al. Post-stroke arm motor telerehabilitation web-based. Int Workshop Virtual Rehabil. 2006:145-8.

170. Piron L, Turolla A, Agostini M, et al. Motor learning principles for rehabilitation: A pilot randomized controlled study in poststroke patients. Neurorehabil Neural Repair. 2010;24:501-8.

171. Popović MD, Kostić MD, Rodić SZ, Konstantinović LM. Feedback-mediated upper extremities exercise: Increasing patient motivation in poststroke rehabilitation. BioMed Res Int. 2014;2014. 
172. Prange GB, Jannink MJ, Stienen AH, van der Kooij H, MJ IJ, Hermens HJ. An explorative, cross-sectional study into abnormal muscular coupling during reach in chronic stroke patients. J Neuroeng Rehabil. 2010;7:14.

173. Qiu Q, Fluet GG, Saleh S, Lafond I, Merians AS, Adamovich SV. Integrated versus isolated training of the hemiparetic upper extremity in haptically rendered virtual environments. Ann Int Con IEEE Eng Med Biol. 2010:2255-8.

174. Quattrocchi G, Greenwood R, Rothwell JC, Galea JM, Bestmann S. Reward and punishment enhance motor adaptation in stroke. J Neurol Neurosurg Psychiatry. 2017;88:730-6.

175. Reinkensmeyer DJ, Maier MA, Guigon E, et al. Do robotic and non-robotic arm movement training drive motor recovery after stroke by a common neural mechanism? Experimental evidence and a computational model. Ann Int Con IEEE Eng Med Biol Soc. 2009;2439-41.

176. Ribeiro Coqueiro P, de Freitas SM, Assuncao e Silva CM, Alouche SR. Effects of direction and index of difficulty on aiming movements after stroke. Behav Neurol. 2014;2014:909182.

177. Robertson JV, Roche N, Roby-Brami A. Influence of the side of brain damage on postural upperlimb control including the scapula in stroke patients. Exp Brain Res. 2012;218:141-55.

178. Rodrigues MR, Slimovitch M, Chilingaryan G, Levin MF. Does the finger-to-nose test measure upper limb coordination in chronic stroke? J Neuroeng Rehabil. 2017;14:6.

179. Rohafza M, Fluet GG, Qiu Q, Adamovich S. Correlations between statistical models of robotically collected kinematics and clinical measures of upper extremity function. Ann Int Con IEEE Eng Med Biol Soc. 2012;2012:4120-3.

180. Rohafza M, Fluet GG, Qiu Q, Adamovich S. Correlation of reaching and grasping kinematics and clinical measures of upper extremity function in persons with stroke related hemiplegia. Ann Int Con IEEE Eng Med Biol Soc. 2014;2014:3610-3.

181. Rohrer B, Fasoli S, Krebs HI, et al. Movement smoothness changes during stroke recovery. J Neurosci. 2002;22:8297-304

182. Rose DK, Winstein CJ. The co-ordination of bimanual rapid aiming movements following stroke. Clin Rehabil. 2005;19:452-62.

183. Rundquist PJ, Dumit M, Hartley J, Schultz K, Finley MA. Three-dimensional shoulder complex kinematics in individuals with upper extremity impairment from chronic stroke. Disabil Rehabil. 2012;34:402-7.

184. Sangole AP, Levin MF. Palmar arch modulation in patients with hemiparesis after a stroke. Exp Brain Res. 2009;199:59-70.

185. Santos GL, Russo TL, Nieuwenhuys A, Monari D, Desloovere K. Kinematic analysis of a drinking task in chronic hemiparetic patients using features analysis and statistical parametric mapping. Arch Phys Med Rehabil. 2018 Mar;99(3):501-11.e4.

186. Schaefer DY, Haaland KY, Sainburg RL, Schaefer SY, Haaland KY, Sainburg RL. Ipsilesional motor deficits following stroke reflect hemispheric specializations for movement control. Brain. 2007;130:2146-58.

187. Schaefer SY, Haaland KY, Sainburg RL. Dissociation of initial trajectory and final position errors during visuomotor adaptation following unilateral stroke. Brain Res. 2009a;1298:78-91.

188. Schaefer SY, Haaland KY, Sainburg RL. Hemispheric specialization and functional impact of ipsilesional deficits in movement coordination and accuracy. Neuropsychologia. 2009b;47:295366. 
189. Senesac CR, Davis S, Richards L. Generalization of a modified form of repetitive rhythmic bilateral training in stroke. Hum Mov Sci. 2010;29:137-48.

190. Sethi A, Stergiou N, Patterson TS, Patten C, Richards LG. Speed and rhythm affect temporal structure of variability in reaching poststroke: A pilot study. J Mot Behav. 2017;49:35-45.

191. Shaikh T, Goussev V, Feldman AG, Levin MF. Arm-trunk coordination for beyond-the-reach movements in adults with stroke. Neurorehabil Neural Repair. 2014;28:355-66.

192. Silveira Fernandes ABG, Oliveira dos Passos J, Paiva de Brito D, Campos TF. Comparison of the immediate effect of the training with a virtual reality game in stroke patients according side brain injury. NeuroRehabilitation. 2014;35:39-45.

193. Sim SM, Oh DW, Chon SC. Immediate effects of somatosensory stimulation on hand function in patients with poststroke hemiparesis: A randomized cross-over trial. Int J Rehabil Res. 2015;38:306-12.

194. Simkins M, Al-Refai AH, Rosen J. Upper limb joint space modeling of stroke induced synergies using isolated and voluntary arm perturbations. IEEE Trans Neural Syst Rehabil Eng. 2014a;22:491-500.

195. Sivan M, Gallagher J, Makower S, et al. Home-based computer assisted arm rehabilitation (hcaar) robotic device for upper limb exercise after stroke: Results of a feasibility study in home setting. J Neuroeng Rehabil. 2014;11:163.

196. Song R, Tong K, Hu X, Zhou W. Myoelectrically controlled wrist robot for stroke rehabilitation. J Neuroeng Rehabil. 2013;10:52.

197. Stewart JC, Gordon J, Winstein CJ. Control of reach extent with the paretic and nonparetic arms after unilateral sensorimotor stroke ii: Planning and adjustments to control movement distance. Exp Brain Res. 2014a;232:3431-43.

198. Subramanian SK, Levin MF. Viewing medium affects arm motor performance in $3 \mathrm{~d}$ virtual environments. J Neuroeng Rehabil. 2011;8:36.

199. Subramanian SK, Lourenco CB, Chilingaryan G, Sveistrup H, Levin MF. Arm motor recovery using a virtual reality intervention in chronic stroke: Randomized control trial. Neurorehabil Neural Repair. 2013;27:13-23.

200. Subramanian SK, Chilingaryan G, Levin MF, et al. Influence of training environment and cognitive deficits on use of feedback for motor learning in chronic stroke. Int Con Virtual Rehabil. 2015;3843.

201. Sukal TM, Ellis MD, Dewald JP. Shoulder abduction-induced reductions in reaching work area following hemiparetic stroke: Neuroscientific implications. Exp Brain Res. 2007;183:215-23.

202. Thielman G, Kaminski T, Gentile AM. Rehabilitation of reaching after stroke: Comparing 2 training protocols utilizing trunk restraint. Neurorehabil Neural Repair. 2008;22:697-705.

203. Thielman G. Insights into upper limb kinematics and trunk control one year after task-related training in chronic post-stroke individuals. J Hand Ther. 2013;26:156-60; quiz 161.

204. Tobler-Ammann BC, de Bruin ED, Fluet MC, Lambercy O, de Bie RA, Knols RH. Concurrent validity and test-retest reliability of the virtual peg insertion test to quantify upper limb function in patients with chronic stroke. J Neuroeng Rehabil. 2016;13:8.

205. Tropea P, Cesqui B, Monaco V, Aliboni S, Posteraro F, Micera S. Effects of the alternate combination of "error-enhancing" and "active assistive" robot-mediated treatments on stroke patients. IEEE J Transl Eng Health Med. 2013;1:2100109. 
206. Turolla A, Daud Albasini OA, Oboe R, et al. Haptic-based neurorehabilitation in poststroke patients: A feasibility prospective multicentre trial for robotics hand rehabilitation. Comput Math Methods Med. 2013;2013:895492.

207. Valdes BA, Glegg SMN, Van der Loos HFM. Trunk compensation during bimanual reaching at different heights by healthy and hemiparetic adults. J Mot Behav. 2016:1-13.

208. van Dokkum L, Mottet D, Bonnin-Koang HY, et al. People post-stroke perceive movement fluency in virtual reality. Exp Brain Res. 2012;218:1-8.

209. van Kordelaar J, van Wegen EE, Kwakkel G. Unraveling the interaction between pathological upper limb synergies and compensatory trunk movements during reach-to-grasp after stroke: A cross-sectional study. Exp Brain Res. 2012;221:251-62.

210. van Meulen FB, Reenalda J, Buurke JH, Veltink PH. Assessment of daily-life reaching performance after stroke. Ann Biomed Eng. 2015;43:478-86.

211. Venkataraman V, Turaga P, Baran M, et al. Component-level tuning of kinematic features from composite therapist impressions of movement quality. IEEE J Biomed Health Inform. 2016;20:14352.

212. Wagner JM, Rhodes JA, Patten C. Reproducibility and minimal detectable change of threedimensional kinematic analysis of reaching tasks in people with hemiparesis after stroke. Phys Ther. 2008;88:652-63.

213. Waller SM, Harris-Love M, Liu W, Whitall J. Temporal coordination of the arms during bilateral simultaneous and sequential movements in patients with chronic hemiparesis. Exp Brain Res. 2006;168:450-4.

214. Willigenburg NW, McNally MP, Hewett TE, Page SJ. Portable myoelectric brace use increases upper extremity recovery and participation but does not impact kinematics in chronic, poststroke hemiparesis. J Mot Behav. 2017;49:46-54.

215. Wittmann F, Held JP, Lambercy O, et al. Self-directed arm therapy at home after stroke with a sensor-based virtual reality training system. J Neuroeng Rehabil. 2016;13:75.

216. Woodbury ML, Anderson K, Finetto C, et al. Matching task difficulty to patient ability during task practice improves upper extremity motor skill after stroke: A proof-of-concept study. Arch Phys Med Rehabil. 2016;97:1863-71

217. Woodbury ML, Howland DR, McGuirk TE, et al. Effects of trunk restraint combined with intensive task practice on poststroke upper extremity reach and function: A pilot study. Neurorehabil Neural Repair. 2009;23:78-91.

218. Wu CY, Chen CL, Tang SF, Lin KC, Huang YY. Kinematic and clinical analyses of upper-extremity movements after constraint-induced movement therapy in patients with stroke: A randomized controlled trial. Arch Phys Med Rehabil. 2007a;88:964-70.

219. Wu CY, Lin KC, Chen HC, Chen IH, Hong WH. Effects of modified constraint-induced movement therapy on movement kinematics and daily function in patients with stroke: A kinematic study of motor control mechanisms. Neurorehabil Neural Repair. 2007b;21:460-6.

220. Wu C, Chou S, Kuo M, Chen C, Lu T, Fu Y. Effects of object size on intralimb and interlimb coordination during a bimanual prehension task in patients with left cerebral vascular accidents. Motor Control. 2008;12:296-310.

221. Wu CY, Chou SH, Chen CL, Kuo MY, Lu TW, Fu YC. Kinematic analysis of a functional and sequential bimanual task in patients with left hemiparesis: Intra-limb and interlimb coordination. Disabil Rehabil. 2009;31:958-66. 
222. Wu CY, Chuang LL, Lin KC, Chen HC, Tsay PK. Randomized trial of distributed constraintinduced therapy versus bilateral arm training for the rehabilitation of upper-limb motor control and function after stroke. Neurorehabil Neural Repair. 2011;25:130-9.

223. Wu CY, Chen YA, Chen HC, Lin KC, Yeh IL. Pilot trial of distributed constraint-induced therapy with trunk restraint to improve poststroke reach to grasp and trunk kinematics. Neurorehabil Neural Repair. 2012a;26:247-55.

224. Wu CY, Chen YA, Lin KC, Chao CP, Chen YT. Constraint-induced therapy with trunk restraint for improving functional outcomes and trunk-arm control after stroke: A randomized controlled trial. Phys Ther. 2012b;92:483-92.

225. Wu CY, Yang CL, Chuang LL, et al. Effect of therapist-based versus robot-assisted bilateral arm training on motor control, functional performance, and quality of life after chronic stroke: A clinical trial. Phys Ther. 2012c;92:1006-16.

226. Wu CY, Yang CL, Chen MD, Lin KC, Wu LL. Unilateral versus bilateral robot-assisted rehabilitation on arm-trunk control and functions post stroke: A randomized controlled trial. J Neuroeng Rehabil. 2013a;10:35.

227. Wu C-Y, Huang P-C, Chen Y-T, Lin K-C, Yang H-W. Effects of mirror therapy on motor and sensory recovery in chronic stroke: A randomized controlled trial. Arch Phys Med Rehabil. 2013b;94:1023-30.

228. Xu R, Johnson MJ. Evaluating motor performance after stroke with a custom mr-conditional upper extremity reach and grasp system. 4th IEEE RAS \& EMBS Int Con Biomed Robot Biomechatron. 2012;1995-2000.

229. Yang Q, Yang Y, Luo J, Li L, Yan T, Song R. Kinematic outcome measures using target-reaching arm movement in stroke. Ann Biomed Eng. 2017;45:2794-803.

230. Yoo DH, Kim SY. Effects of upper limb robot-assisted therapy in the rehabilitation of stroke patients. J Phys Ther Sci. 2015;27:677-9.

231. Zollo L, Gallotta E, Guglielmelli E, Sterzi S. Robotic technologies and rehabilitation: New tools for upper-limb therapy and assessment in chronic stroke. Eur J Phys Rehabil Med. 2011a;47:22336.

232. Zollo L, Rossini L, Bravi M, Magrone G, Sterzi S, Guglielmelli E. Quantitative evaluation of upperlimb motor control in robot-aided rehabilitation. Med Biol Eng Comput. 2011b;49:1131-44.

233. Li J, Pan B, Jin T, et al. A single task assessment system of upper-limb motor function after stroke. Technol Health Care. 2016;24 Suppl 2:S707-15.

234. Liu W, McCombe Waller S, Kepple TM, Whitall J. Compensatory arm reaching strategies after stroke: Induced position analysis. J Rehabil Res Dev. 2013;50:71-84.

235. Yu L, Xiong D, Guo L, Wang J. A compressed sensing-based wearable sensor network for quantitative assessment of stroke patients. Sensors. 2016;16:202.

236. Zhang Z, Fang Q, Gu X. Objective assessment of upper-limb mobility for poststroke rehabilitation. IEEE Trans Biomed Eng. 2016;63:859-68.

237. Kung PC, Lin CC, Ju MS. Neuro-rehabilitation robot-assisted assessments of synergy patterns of forearm, elbow and shoulder joints in chronic stroke patients. Clin biomech. 2010;25:647-54.

238. Knaut LA, Subramanian SK, McFadyen BJ, Bourbonnais D, Levin MF. Kinematics of pointing movements made in a virtual versus a physical 3-dimensional environment in healthy and stroke subjects. Arch Phys Med Rehabil. 2009;90:793-802. 
239. Wu C-Y, Liing R-J, Chen H-C, Chen C-L, Lin K-C. Arm and trunk movement kinematics during seated reaching within and beyond arm's length in people with stroke: A validity study. Phys Ther. 2014;94:845-56.

240. Harris C, Wolpert DM. Signal-dependent noise determines motor planning. Nature. 1198;394:7804.

241. Fluet M-C, Lambercy O, Gassert R. Upper limb assessment using a virtual peg insertion test. IEEE Int Con Rehabil Robot. 2011;2011:5975348.

242. Reinkensmeyer DJ, Iobbi MG, Kahn LE, Kamper DG, Takahashi CD. Modeling reaching impairment after stroke using a population vector model of movement control that incorporates neural firing-rate variability. Neural Comput. 2003;15:2619-42.

243. Hogan N, Sternad D. Sensitivity of smoothness measures to movement duration, amplitude, and arrests. J Mot behav. 2009;41:529-34.

244. Balasubramanian S, Melendez-Calderon A, Burdet E. A robust and sensitive metric for quantifying movement smoothness. IEEE Trans Biomed Eng. 2012;59:2126-36.

245. Balasubramanian S, Melendez-Calderon A, Roby-Brami A, Burdet E. On the analysis of movement smoothness. J Neuroeng Rehabil. 2015;12:112.

246. Balasubramanian S. Matlab source code for spectral arc length (sparc). 2016

247. Cirstea MC, Levin MF. Compensatory strategies for reaching in stroke. Brain. 2000;123 (Pt 5):940-53. 



\section{Measures of interjoint coordination post-stroke across different upper limb movement tasks}

A. Schwarz

J.M. Veerbeek

J.P.O. Held

J.H. Buurke

A.R. Luft 


\subsection{Abstract}

Background - Deficits in interjoint coordination, such as the inability to move out of synergy, are frequent symptoms in stroke subjects with upper limb impairments that hinder them from regaining normal motor function. Kinematic measurements allow fine-grained assessment of movement pathologies, thereby complementing clinical scales, like the Fugl-Meyer Motor Assessment of the Upper Extremity (FMMA-UE). The study goal was to investigate the effects of the performed task, the tested arm, the dominant affected hand, upper limb function, and age on spatiotemporal parameters of the elbow, shoulder, and trunk. The construct validity of the metrics was examined by relating them with each other, the FMMA-UE, and its arm section.

Methods - This is a cross-sectional observational study including chronic stroke patients with mildto-moderate upper limb motor impairment. Kinematic measurements were taken using a wearable sensor suit while performing four movements with both upper limbs: (1) isolated shoulder flexion, (2) pointing, (3) reach-to-grasp a glass, and (4) key insertion. The kinematic parameters included the joint ranges of shoulder abduction/adduction, shoulder flexion/extension, and elbow flexion/extension; trunk displacement; shoulder-elbow correlation coefficient; median slope; and curve efficiency. The effects of the task and tested arm on the metrics were investigated using a mixed model analysis. The validity of metrics compared to clinically measured interjoint coordination (FMMA-UE) was done by correlation analysis.

Results - Twenty-six subjects were included in the analysis. The movement task and tested arm showed significant effects $(\mathrm{p}<0.05)$ on all kinematic parameters. Hand dominance resulted in significant effects on shoulder flexion/extension and curve efficiency. The level of upper limb function showed influences on curve efficiency and the factor age on median slope. Relations with the FMMA-UE revealed the strongest and significant correlation for curve efficiency $(\mathrm{r}=0.75)$, followed by shoulder flexion/extension ( $\mathrm{r}=0.68)$, elbow flexion/extension $(\mathrm{r}=0.53)$ and shoulder abduction/adduction $(\mathrm{r}=0.49)$. Curve efficiency additionally correlated significantly with the arm subsection focusing on synergistic control $(\mathrm{r}=0.59)$.

Conclusion - Kinematic parameters of the upper limb after stroke were influenced largely by the task. These results underpin the necessity to assess different relevant functional movements close to real world conditions rather than relying solely on clinical measures.

Study registration - Clinicaltrials.gov Identifier NCT03135093 and BASEC-ID 2016-02075.

Keywords - Upper extremity; stroke; biomechanical phenomena; interjoint coordination; kinematics. 


\subsection{Introduction}

Incidences of upper limb impairments after stroke have been reported in $48 \%$ to $85 \%$ of acute stroke patients (Jorgenson et al., 1999; Persson et al., 2012). Acute deficits might include paresis, ataxia and loss of sensory function (Yew et al., 2009). The course of recovery from these impairments varies from complete restoration to different degrees of compensatory adaptation (Levin et al., 2009; Bernhardt et al., 2017). Throughout the course, deficits in interjoint coordination have been described as a key feature in stroke-related dysfunctions that is characterized by the reappearance of primitive movement synergies and the presence of joint coupling (Krakauer and Carmichael, 2017). Interjoint coordination has been defined as the process to spatially and temporally arrange the degrees of freedom (DOF) needed to achieve the movement goal (Tomita et al., 2017) and is closely linked to the concept of synergies (Santello et al., 2015; McMorland et al., 2015; Roh et al., 2013). Based on two principal synergies, the flexor and the extensor synergy, pathological stereotypical coupling between two or more DOF has been observed as a phenotype of the loss of interjoint coordination after stroke. A loss of interjoint coordination is associated with weakness (Sukal et al., 2007) and spasticity (Allison et al., 2015) along the time course after stroke (Levin, 1996; Cirstea, 2003), leading to learned bad or non-use in daily life (Taub et al., 2006; Raghavan et al., 2015). Determining the level of interjoint coordination and associated motor dysfunction of stroke-related movement disabilities is critical to improve our understanding and expand interventional strategies to minimize long-term consequences due to stroke.

Interjoint coordination after stroke is often assessed by the Fugl-Meyer Motor Assessment of the Upper Extremity (FMMA-UE). This clinical assessment evaluates volitional movement control of the upper limb in a hierarchical manner from proximal to distal segments (FuglMeyer et al., 1975) and by taking into account the within synergy, mixed synergy and out of synergy movement patterns as proposed by Thomas E. Twitchell (Twitchell, 1951) and Signe Brunnstrom (Brunnstrom, 1966, 1970). Although the FMMA-UE has been attested high quality in clinimetric properties (Gladstone et al., 2002), some limitations need to be considered in terms of the measurement construct being used. First, items of the FMMA-UE are assessed on a three-point ordinal scale ("not", "partial", "fully") and the "partial" category is very broad. An evaluation of "partial" movement achievement includes limitations in active range of motion or movement deviations, such as shoulder abduction or elbow flexion during shoulder flexion, that can range from small to exaggerated differences and cannot be differentiated further. This level of evaluation of movement quality does not allow to differentiate between physiological and pathological movement behavior (Kwakkel et al., 
2017). Second, a full score in FMMA-UE cannot be directly related to complete restitution since deviations in movement kinematics and limitations in daily life might still be present (Thrane et al., 2019). Third, the FMMA-UE assesses mostly abstract movements and limb postures, based on empirically derived stroke recovery stages, that have little to no relevance to the subject's movements in daily life. Considering the widespread and recommended usage of the FMMA-UE as a primary outcome measure stroke research trials (Santisteban et al., 2016; Kwakkel et al., 2019; Burridge et al., 2019; Subramanian et al., 2020) and the overall neutral results of most stroke rehabilitation trials (Corbetta et al., 2015; Eraifrej et al., 2017; Veerbeek et al., 2017), the question, in how far this outcome can sensitively capture changes on the body function level when performing daily life tasks, cannot be omitted.

The introduction of modern technology opened new avenues for assessments of motor function. Upper limb kinematic motion analysis in the stroke population have been performed with $2 \mathrm{D}$ and $3 \mathrm{D}$ set-up conditions for assessing a large number of different kinematic outcome parameters in predominantly pointing or reach-to grasp tasks (Schwarz et al., 2019). Kinematic parameters measure body functions and thereby characterize aspects of movement control, such as interjoint coordination. Outcome measures to quantify upper limb interjoint coordination include spatial measures of active range of motion in shoulder and elbow, and trunk displacement (van Kordelaar et al., 2012), that have been attested sufficient validity and reliability in 3D pointing tasks (Subramanian et al., 2010; Massie et al., 2011; Massie et al., 2014; Wu et al., 2014). Measures of interjoint coordination, relating at least two DOF, ranged from angle-angle plots (Beer et al., 2007; Woodbury et al., 2009; Alt-Murphy et al., 2011), correlation analysis (Yang et al., 2017), slope statistics (Banina et al., 2017), ratio or index measures (Cirstea et al., 2007; Levin et al., 2016) to mathematically more complex parameters, such as functional Principal Component Analysis (PCAs) (van Kordelaar et al., 2013) or approximate entropy metrics (Sethi et al., 2017). Parallel to this, movement timing or workspace measures, such as circle size area (Sukal et al., 2007; Ellis et al., 2016; Krabben et. al., 2011) provide indirect measures as result of pathological synergies. Taken together, the variety of metrics identified for evaluating interjoint coordination illustrates the wide context and aspects of this movement construct and tight connection between the movement characteristics and the chosen metric, as for example the circle size area in a circle drawing task (Houwink et al., 2013). Considering this state of the art in upper limb kinematic assessments, it could be proposed that research on interjoint coordination would profit from task-independent metrics that could be evaluated in various tasks and settings thereby allowing for comparability, especially for pooling in meta-analysis. 
In this study, first, it was questioned whether kinematic parameters representing aspects of interjoint coordination in the shoulder-elbow-trunk complex are different with respect to different movement tasks and the arm being tested by considering the dominant affected side, the upper limb function, and age as covariates. Second, it was examined whether statistically significant correlations can be found between each of the kinematic parameters of the affected side, the FMMA-UE full score and the FMMA-UE arm subscale that evaluates the shoulder-elbow-trunk complex according to the synergy-concept. The findings will provide new insides into characteristics interjoint coordination in different functional and nonfunctional upper limb movements after stroke, propose kinematic parameters to quantify spatiotemporal aspects of interjoint coordination, and as a long-term goal, support the establishment of feasible and repeatable qualitative kinematic assessments in close relation to real-world functional activities.

\subsection{Methods}

A prospective cross-sectional study was performed at the rehabilitation clinic Cereneo, Vitznau Switzerland to explore the relationship between upper limb function and activity as measured by clinical assessments and by a wearable motion capture system. The study protocol was approved by the Cantonal Ethics Committee Northwest and Central Switzerland (BASEC-ID: 2016-02075) and prospectively registered in ClinicalTrials.gov (NCT03135093). Between July 2017 and October 2019, 523 patients from the stroke research register of the Department of Neurology, University Hospital Zurich (Zurich, Switzerland) were screened by telephone and onsite screening.

\subsubsection{Study participants}

Subjects were deemed eligible when they met the following inclusion criteria: (1) >6 months post unilateral stroke (hemorrhage or ischemic); (2) at least 18 years of age; and (3) upper limb motor impairment, but at least partial able to lift the arm against gravity $\left(>30^{\circ}\right.$ of shoulder flexion), and to flex and extend the fingers for basic grasping. The exclusion criteria were (1) an increased upper limb muscle tone with limitations in range of motion (modified Ashworth Scale $[\mathrm{MAS}] \geq 3$ ); (2) severe sensory deficits in the upper limb (Erasmus modifications to the revised Nottingham Sensory Assessment [EmNSA] of 0 in one of the test regions); (3) a preexisting orthopedic or neurological disease affecting movements of the upper limb; (4) contraindications on ethical grounds, e.g. person who are decisionally impaired; (5) 
known or suspected non-compliance; or (6) severe communication or cognitive deficits that cause inability to follow the study procedures as determined by the Montreal Cognitive Assessment [MoCA] $\leq 20$ points (Dong et al., 2013). The MAS (Bohannon and Smith, 1987) and the EmNSA (Stolk-Hornsveld et al., 2006) were performed with the participant in supine position. The EmNSA (was evaluated for the surface, pinprick, sharp-blunt and proprioceptive discrimination in both arms. Each participant gave written informed consent according to the Declaration of Helsinki and the Swiss regulatory authorities.

\subsubsection{Study experiments}

An experienced research therapist performed all study experiments during a singleday measurement at the rehabilitation clinic cereneo (Vitznau, Switzerland). The study experiments started after onsite screening and informed consent with setting up the wearable kinematic measurement system. When being acquired with the system, the participant performs the FMMA-UE and a set of daily living activities with both upper limbs. The less-affected side was assessed to determine the close to physiological movement behavior on the best-available level in delineation to pathological movement behavior of the affected upper limb during functional and non-functional activities.

\subsubsection{Measurement system}

A portable and wireless sensor-based motion capture system was used to capture upper limb kinematics (Xsens MVN Awinda, Xsens Technologies, the Netherlands). The system consists of 17 inertial measurement units (IMU), a receiver station, and attachment equipment (MVN Manual, 2018). The nine IMUs of the upper body used in this analysis, were fixated on a T-Shirt above both scapulae with the sensors' $\mathrm{x}$-axes parallel to the spina scapulae and above the sternum with the sensor aligned with the $\mathrm{x}$-axis, as illustrated in Figure 3.1. The upper extremity IMUs were mounted with elastic Velcro-straps on the upper arm above the lateromedial part of the humerus bone, around the distal radioulnar joint, three fingers above the wrist, and on the dorsal palm of the hand by use of a palm glove or medical tape in case of the glove was not fitting. Each IMU contains 3D linear accelerometers, 3D rate gyroscopes, 3D magnetometers, and a battery. Combined with information of the subjects' body measures into a biomechanical model, the data of 3D angular velocity, 3D acceleration, $3 \mathrm{D}$ earth magnetic field, and atmospheric pressure allows stable $3 \mathrm{D}$ orientation for human kinematic motion analysis (MVN software, 2018). The kinematic data is sampled at $60 \mathrm{~Hz}$. The accuracy of the system to measure each body segments' position has been reported as 
approximately $5 \mathrm{~mm}$ and orientation with a measurement error of 3 degrees (Roetenberg et al., 2007a; Roetenberg et al., 2007b). The system was previously validated with a camerabased system (Optotrak) demonstrating comparable results (Robert-Lachaine et al., 2017) and additionally investigated for intra- and interrater reliability with fair-to-excellent results, even when being used by clinicians with no experience in applying motion capture technologies (Al-Amri et al., 2018).

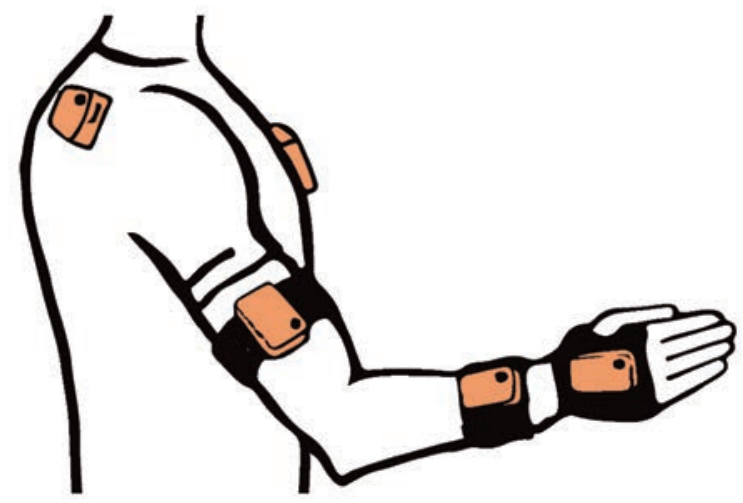

Figure 3.1. Measurement system set-up.

Setting up the system for each participant included taking body measures, such as body height, shoulder height (distance from the ground to top of acromion) and shoulder width (distance between the right and the left lateral boarder of the acromion), the sensor attachment and a calibration procedure, that consisted of standing in neutral position at the calibration spot, walking three meters and return to start. The whole procedure took about 15 to 20 minutes and was completed when the subject returned back to neutral pose standing at the calibration spot. The measurements of all subjects were performed in an upright sitting position on an armless chair in the same examination room of the rehabilitation clinic, as well as position and orientation of the subject. This allowed to control for possible external inferences, that could affect the sensor data of the IMUs, such as electric leads.

\subsubsection{Movement tasks}

The selected movements consisted of four different discrete movement tasks: (1) isolated shoulder flexion, (2) pointing ahead, (3) reach-to-grasp a glass, and (4) key insertion into a lock. The selection was based on the shared upper limb workspace along the sagittal plane and discrete reaching movement while discriminating variations non-functional and functional 
movements with and without grasp contact in alliance with existing upper limb movement (Schambra et al., 2019) and grasp taxonomies (Feix et al., 2016). An overview of the movement tasks including characteristics such as contact and the underlying motion primitives is provided in Table 3.1. Each movement task was demonstrated and instructed verbally, including demotrials, if necessary. The movement start was defined by a flick on one of the sensors. After task completion, the subjects were asked to return to the start position. For the analysis, the

Table 3.1. Upper limb movement task characteristics

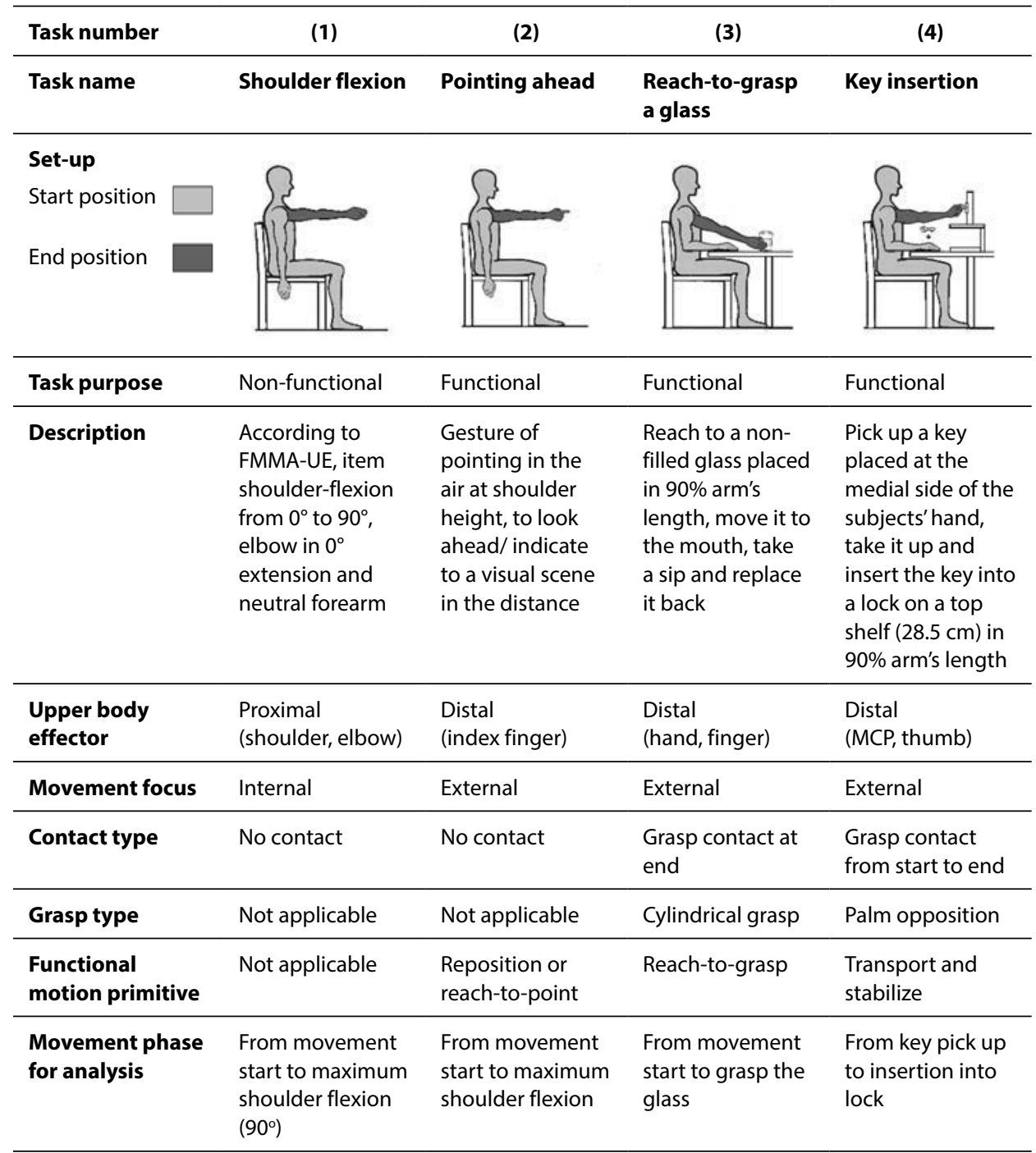

FMMA-UE, Fugl-Meyer Motor Assessment of the Upper Extremity; MCP, metacarpo-phalangeal joint. 
maximum shoulder flexion angle and/or the maximum distance of the hand-IMU positional data along the $\mathrm{x}$-axis defined the movement end. The chair had a standard seat height of 46 $\mathrm{cm}$ with a back support $51 \mathrm{~cm}$ height with a backward inclination that was counterbalanced by fixating a tight pillow at the back of the chair. The table was height-adjustable to allow a subject-specific set up of $0^{\circ}$ in all axis of the shoulder, $90^{\circ}$ of elbow flexion, and the hand pronated on the table. Subjects were instructed to perform the task at a comfortable speed while keeping contact with the back of the chair. This instruction was given once at the beginning to not interfere profoundly with the natural movement behavior. Three to six repetitions were performed with each upper limb to include at least three successful trials in the data analysis (Alt-Murphy et al., 2018), starting with the less-affected and followed by the affected side.

\subsubsection{Outcome measures}

The recorded kinematic measures were segmented by movement trial based on the flip-signal and the maximal target angle and stored in mvnx-files for data transferring and processing in MATLAB (The MathWorks Inc., Natick, MA, USA). For each movement task, participant and tested upper limb, the shoulder and elbow angles and the positional data of the trunk sensor of all repetitions were extracted for analysis. The kinematic parameters of interest consisted of spatial and spatiotemporal measures.

\subsubsection{Spatial parameters}

Spatial measures included joint angle ranges in degrees around one rotation axis and trunk displacement in $\mathrm{mm}$. Each joint can be expressed in $6 \mathrm{DOF}$ around the orthogonally arranged rotation axis, were one joint angle is defined by a joint rotation as the orientation of a distal segment with respect to a proximal segment. Joint rotations are calculated using the Euler sequences ZXY and XZY by the MVN software (MVN Manual, 2018) based on the coordinate system agreed by the International Society of Biomechanics (ISB) (Wu et al., 2014). All angles follow the ISB Euler angle extractions of $\mathrm{Z}$ (flexion/extension), $\mathrm{X}$ (abduction/adduction) $\mathrm{Y}$ (internal/external rotation), except for the shoulder joint, where the Euler sequence XZY is used. The definitions of the origins of the segments are somewhat different from markerbased recommendations, since MVN uses motion tracker placed on the segment, rather than markers placed on bony landmarks close to the joint origin (MVN Manual, 2018).

The range of motion was defined by calculating the minimum and maximum angle for all data points from movement onset to end (van Meulen et al., 2015). The standard deviations of the minimum and maximum joint angle were calculated as a measure of variability. 
For the purpose of this study to evaluate interjoint coordination in the shoulder-elbow complex, shoulder flexion/ extension, shoulder abduction/adduction and elbow flexion/ extension were captured and analyzed. Even though shoulder rotational movements are an important component of the upper limb, they were not considered in this study, since measurement accuracy of rotations around the transversal plane were associated with the largest measurement error ranging from 16 to $34^{\circ}$ (Warmsley et al., 2018). The challenge to measure rotational movements on the transversal plane might be related to the larger differences between soft-tissue and bone motions during rotation. Elbow flexion/extension was determined by rotation around the $\mathrm{z}$-axes, where elbow extension was represented by $0^{\circ}$ and positive values indicating flexion of the elbow. Shoulder flexion-extension was defined as an elevation parallel to the sagittal plane and angles that rotate around the $\mathrm{z}$-axis. Shoulder abduction-adduction was defined as an elevation on the frontal plane and rotates around $\mathrm{x}$-axes of the shoulder joint. Positive values indicate shoulder flexion or abduction and negative values shoulder extension or adduction.

In contrast to ISB descriptions of the shoulder with the thorax, clavicle, scapula and humerus, the MVN model does not define the thorax segment nor the clavicle. The MVN model splits the thorax region into spine segments (MVN Manual, 2018). In alliance with other studies in the field, trunk motions were simplified to trunk displacement as defined by changes in position and orientation of the sternum sensor between movement onset and end (Subramanian et al., 2010). The change in trunk displacement was calculated by subtracting the mean of the first 10 data points from the other position values in $\mathrm{x}^{-}, \mathrm{y}^{-}$and z-direction and were summarized by:

$$
\text { Trunk displacement }=\sqrt{\left(T x^{2}+T y^{2}+T z^{2}\right)}
$$

where $\boldsymbol{T} \boldsymbol{x}$ includes frontal displacement, $\boldsymbol{T} \boldsymbol{y}$ sideway displacement and $\boldsymbol{T} \boldsymbol{z}$ displacement in rotation.

\subsubsection{Spatiotemporal parameters of shoulder-elbow coordination}

Angle-angle plots of shoulder and the elbow flexion angle for each timeframe of the movement were derived to qualitatively analyze interjoint coordination and coupling between shoulder and elbow flexion/extension in reaching, as illustrated in Figure 3.2. For each movement repetition per participant, the elbow and shoulder angles were set to $0^{\circ}$ or $90^{\circ}$ according to the related starting position and time-normalized with respect to the mean trial length to enable comparability. 
A shoulder elbow correlation coefficient was calculated to quantify the relationship between shoulder flexion/extension $(\boldsymbol{S F})$ and elbow flexion/extension $(\boldsymbol{E F})$ in the following equation:

$$
r=\frac{\sum_{m} \sum_{n}\left(S F_{m n}-S F_{\text {mean }}\right)\left(E F_{m n}-E F_{\text {mean }}\right)}{\sqrt{\left(\sum_{m} \sum_{n}\left(S F_{m n}-S F_{\text {mean }}\right)^{\wedge} 2 \sum_{m} \sum_{n}\left(E F_{m n}-E F_{\text {mean }}\right)^{\wedge} 2\right)}}
$$

where $S F_{\text {mean }}=\operatorname{mean}^{2}(\boldsymbol{S F})$, and $E F_{\text {mean }}=\operatorname{mean}^{2}(\boldsymbol{E F})$.

In the case of isolated joint movements, a low correlation coefficient highlights the ability to uncouple joint movements, whereas a coupling relationship was detected, if the change in movement direction of two segment occurred at the same time. In isolated joint motions of task (1), a well-coordinated movement with a constantly extended elbow would results in a correlation coefficient close to 0 , whereas pathological coupled movements would result in a higher correlation coefficient, according to the hypothesis of voluntary joint control. Reaching out for an object on a table is likely to start from an elbow flexed position then requires the elbow to extend while the shoulder is being elevated, so that a negative correlation would be expected for physiological movement and conversely a low correlation in case of pathological coupling with remaining elbow flexion while reaching out.

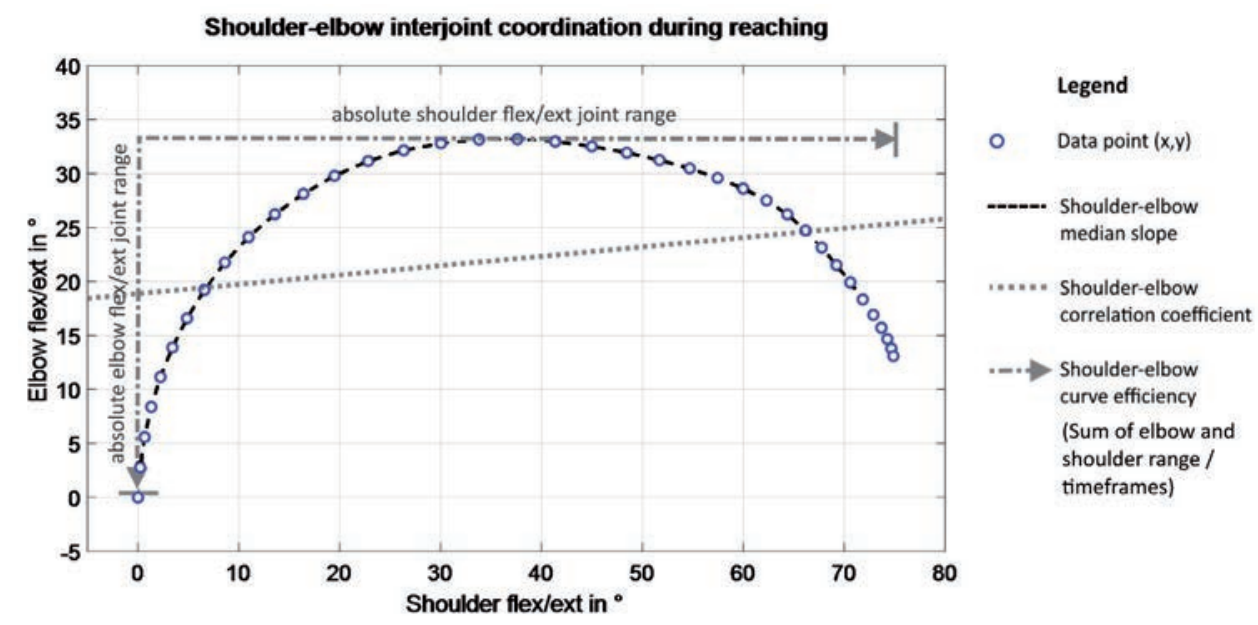

Figure 3.2. Shoulder-elbow coordination measures.

Shoulder-elbow median slope was defined by the slopes connecting the data points of elbow-shoulder angle-angle plots as depicted in Figure 3.2. The mean slope between elbow flexion/extension and shoulder flexion/extension was used to assess interjoint coordination 
by Banina and colleagues (Banina et al., 2017). In this present study the median slope was selected instead of the mean slope to account the non-linearity of angle-angle curves, especially in task (2), (3) and (4). The slope between shoulder and elbow changes per timeframe ranges from positive to negative infinite values representing the gradient of the curve.

Shoulder-elbow curve efficiency is included to quantify the maximum movement execution in the target DOF for the movement. It was defined by the sum of absolute joint range in shoulder flexion/extension and elbow flexion/extension, as visualized in Figure 3.2, divided by the number of data points of the movement, to quantify the amount of both joint ranges in reaching. The sum of absolute joint ranges was normalized with respect to the number of frames, to include temporal efficiency of the movement. For isolated joint movements, such as in task (1), the absolute range in elbow flexion/extension is subtracted from the absolute range in shoulder flexion/extension, divided by the number of timeframes. For the other movement tasks, the absolute ranges in elbow and shoulder flexion/extension were summed to quantify the upper limb movement magnitude during reaching. Values are given in degrees per frame with higher values representing more efficient movement activation to reach the movement goal.

\subsubsection{Clinical measurements}

The FMMA-UE was collected as a clinical stroke-specific measurement to evaluate upper limb motor impairment (Fugl-Meyer et al., 1975). The FMMA-UE is hierarchically composed; starting with assessing reflex appearance and primitive synergy patterns followed by within- to out-of-synergy movements in the arm subscale, based on the assumption, that recovery "follows a definable stepwise course". The FMMA-UE is partitioned into four sections, "upper extremity", "wrist", "hand", and "coordination and speed", as differences in recovery in each subscale could be independent from each other. Each test item is rated based on the best performance with the full FMMA-UE score ranging from zero to 66. For the purpose of this study, upper limb functionality subgroups were considered based on Hoonhorst and colleagues, who stratified FMMA-UE scores according to upper limb capacity measures that include grasping and displacement movements. With this subgroup selection, it was intended to investigate differences with respect to the subjects' capacity in grasping performance. The information on hand dominance was obtained by asking the individual which hand he or she preferred to use for writing and throwing a ball prior to the stroke. 


\subsubsection{Statistical analysis}

The statistical analysis was performed using Matlab (MATLAB version 2016b, The Mathwork, Natick, MA) and SPSS (SPSS version 26.0, IBM Corp., Armonk, N.Y., USA). Spatial measures of joint ranges in elbow flexion/extension, shoulder flexion/extension, shoulder abduction/adduction were presented in absolute range of motion from minima to maxima with the corresponding standard deviations. Trunk displacement was given by absolute displacement from minima to maxima in $\mathrm{mm}$. Spatiotemporal measures of shoulder-elbow coordination included the correlation coefficients ' $r$ ', the median slope, and the curve efficiency. All kinematic outcome parameters were explored for determining normal distribution in histograms and QQ-plots. Descriptive statistics of the kinematic measures were summarized for all subjects and each task and tested limb separately.

A linear mixed model analysis was performed for each kinematic parameter to account for mixed effects in a repeated measurement design. Each kinematic metric was treated as a dependent variable with respect to the independent fixed factors movement task (shoulder flexion, pointing, reach-to-grasp a glass, key insertion), the tested arm (affected, less-affected side), dominant hand is the affected hand (yes, no), the upper limb functionality group, as assessed with the FMMA-UE (32-47 points, "limited"; 48-52 points, "notable"; 53-66 points "full") (Hoonhorst et al., 2014) and age ( $\leq 55$ years and $\geq 56$ years) (Kwakkel et al., 2017).

The relationship between clinically measured impairment and kinematic measures was examined by Spearman rank correlations. To evaluate the relationship between shoulderelbow coordination, as measured in the FMMA-UE arm subsection when compared to spatiotemporal measures, the Spearman rank correlation was used. According the COSMIN guidelines, correlations between two measures of the same construct should be $r \geq 0.5$, correlations of related measures $r=0.3-0.5$ and correlations of unrelated constructs $r<0.3$ (Prinsen et al., 2018). All statistical tests were performed at a significance level of 5\%.

\subsection{Results}

A total of 28 stroke subjects were included in the study of which 26 were included in the data analysis. The study flow of participant inclusion is shown in Figure 3.3. The participant characteristics are summarized in Table 3.2. The study sample represents 26 mild to moderately impaired chronic stroke subjects of whom fourteen subjects were affected in their dominant upper limb. Seventeen subjects of the 26 included showed some resistance 
Stroke subjects potentially eligible between 2016 and $2019(\mathrm{~N}=523)$ (research register of the Department of Neurology, University Hospital Zurich and University of Zurich, Zurich, Switzerland)

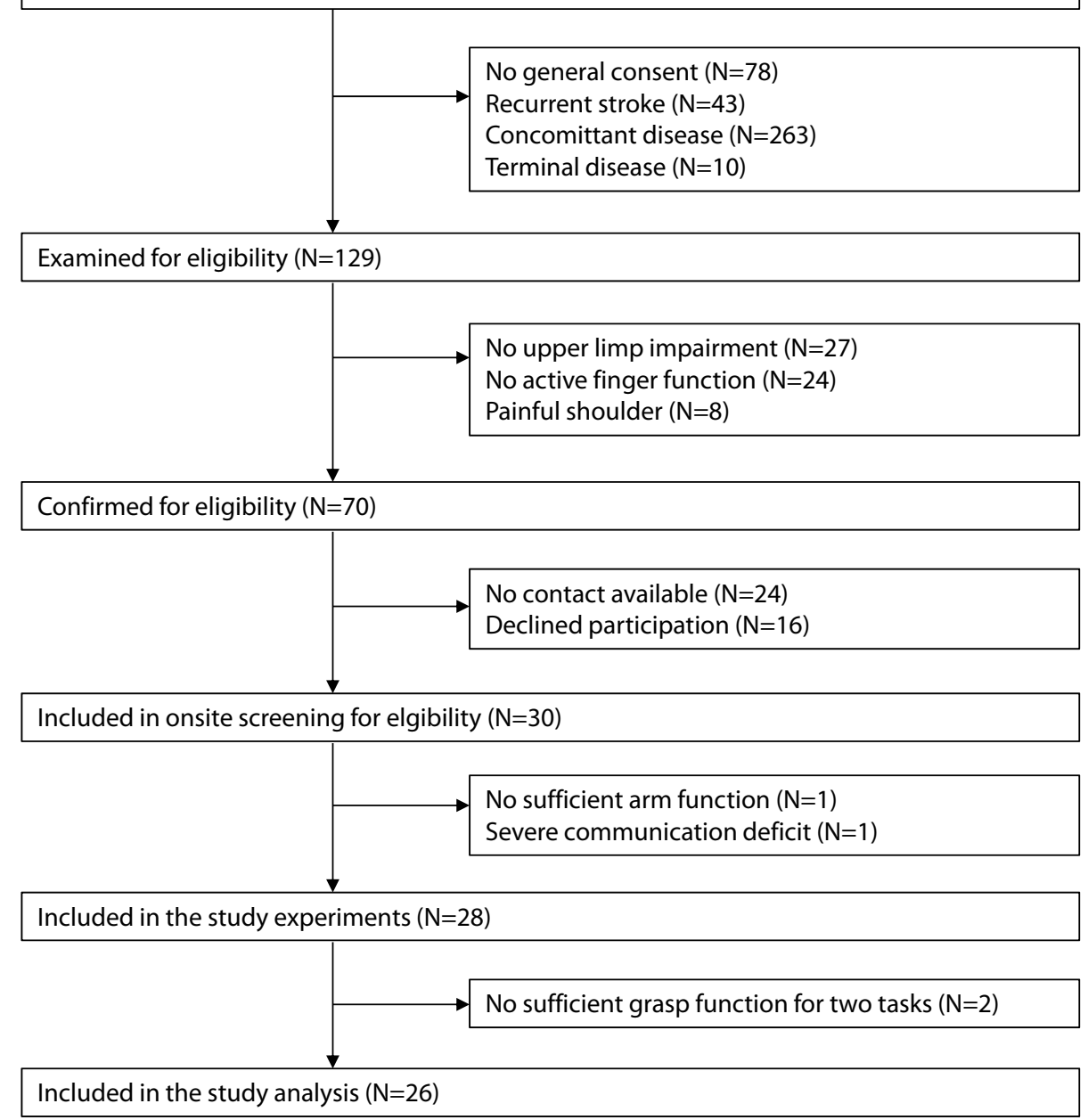

Figure 3.3. Flow of study participants.

against passive movement in at least one of the tested muscles, as defined by a MAS score between 1 and 2. Sensory function was somewhat impaired in 21 subjects as determined by the EmNSA ranging from 29 to 40 points in the affected upper limb. 
Table 3.2. Study participant characteristics

\begin{tabular}{|c|c|}
\hline Characteristic & Total $(\mathrm{N}=26)$ \\
\hline Gender, female/male & $9 / 17$ \\
\hline Mean age (SD), years & $62.19(12.10)$ \\
\hline Mean body height (SD), cm & $173.81(10.94)$ \\
\hline Mean BMI (SD), kg/m² & $26.97(4.23)$ \\
\hline Paretic body side, left/right & $13 / 13$ \\
\hline Months since stroke * & $20.50(12-34)$ \\
\hline Initial stroke severity NIHSS * & $8(6-11)$ \\
\hline $\operatorname{MoCA}(0-30) *$ & $27(24-28)$ \\
\hline MAS sum of the upper extremity $(0-14) *+$ & $1.75(0.25-3)$ \\
\hline Shoulder internal rotator muscles $(\mathrm{N})^{\dagger}$ & 11 \\
\hline Biceps brachii muscle $(\mathrm{N})^{+}$ & 18 \\
\hline 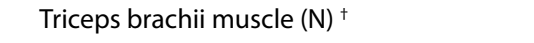 & 3 \\
\hline Wrist flexor muscles $(\mathrm{N})^{\dagger}$ & 6 \\
\hline Wrist extensor muscles $(\mathrm{N})^{+}$ & 4 \\
\hline Finger flexor muscles $(\mathrm{N})^{\dagger}$ & 4 \\
\hline Finger extensor muscles $(\mathrm{N})^{+}$ & 5 \\
\hline EmNSA-UE $(0-40) *$ & $38(36-39)$ \\
\hline FMMA-UE $(0-66) *$ & $47.50(40.25-55.00)$ \\
\hline FMMA-UE arm subsection $(0-36)^{*}$ & $26(22.00-29.75)$ \\
\hline FMMA-UE wrist subsection (0-10) * & $6(6.00-7.75)$ \\
\hline FMMA-UE hand subsection (0-14) * & $11(9.00-14.00)$ \\
\hline FMMA-UE coordination subsection (0-6) * & $4(3.25-5.00)$ \\
\hline
\end{tabular}

BMI, Body Mass Index; EmNSA, Erasmus modified version of the Nottingham Sensory Assessment; FMMAUE, Fugl-Meyer Motor Assessment of the Upper Extremity; MAS, modified Ashworth Scale; MoCA, Montreal Cognitive Assessment; NIHSS, National Institutes of Health Stroke Scale; L, left; SD, standard deviation. ${ }^{*}$ Indicates that values are presented in median (interquartile range); ${ }^{\dagger}$ Indicates MAS scores between 1 and 2 for the tested muscle.

\subsubsection{Kinematic characteristics per task of the affected and non-affected side}

Overall, 468 kinematic datasets per arm were included in the analysis representing 26 stroke subjects when performing four upper limb movement tasks. The observed QQ-Plots for the kinematic parameters did not lead to reject the assumption of normal distribution in the analyzed data. The spatial measures of joint ranges in elbow flexion/extension, shoulder flexion/extension, shoulder abduction/adduction and trunk displacement are summarized for all subjects, each movement task, and affected (red-colored) and less-affected upper limb (blue-colored) in Figure 3.4(A)-(D). Each boxplot illustrates the median, the upper and lower quartile, the minimum and maximum, as well as outliers shown as a red plus for each of the spatial measures. Different ranges across the spatial measures can be seen between the tasks. While increased trunk motions are shown in $4(\mathrm{C})$ and (D) when compared to $4(\mathrm{~A})$ 
(A)

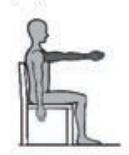

(B)

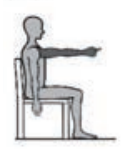

(C)

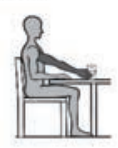

(D)

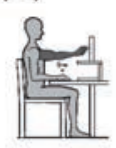

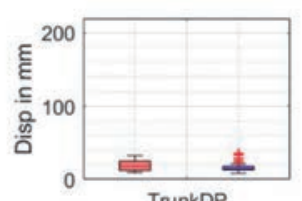

TrunkDP
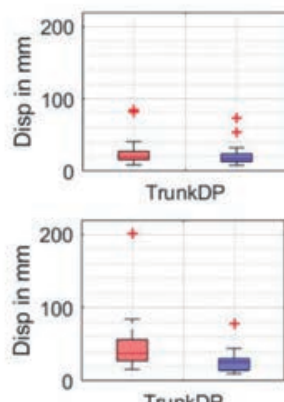

TrunkDP

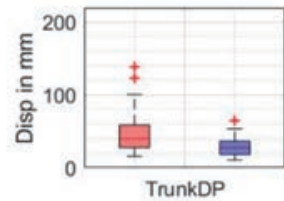

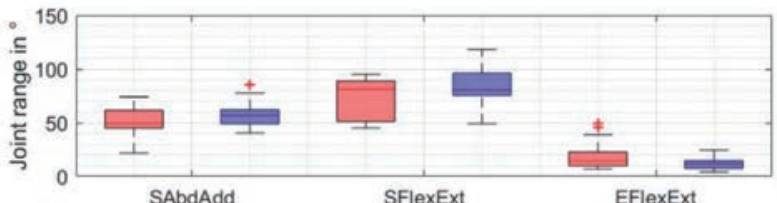
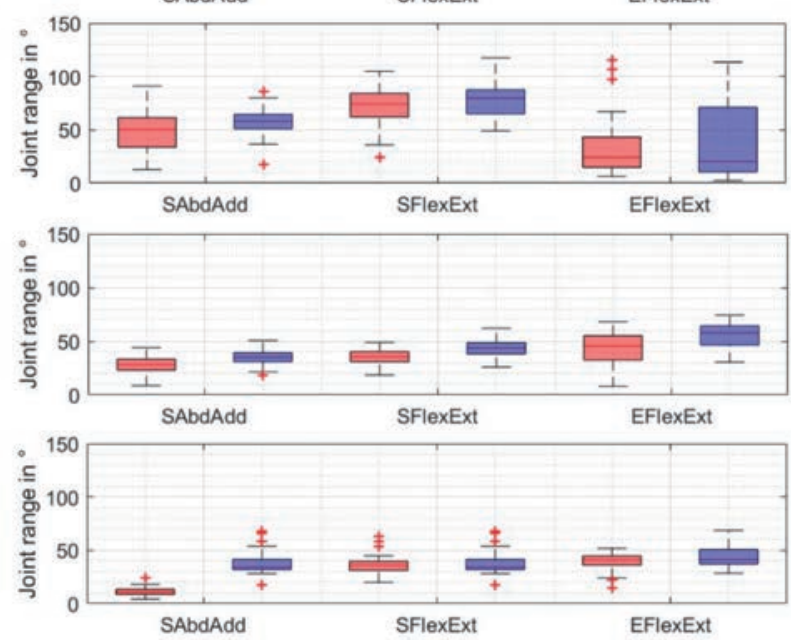

Affected body side

Less-affected body side

Affected body side

$\square$

Start position

Target position

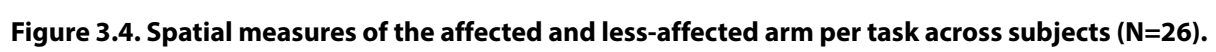

AS, affected side; EFlexExt, elbow flexion/extension; LAS, less-affected side; SFlexExt, shoulder flexion/extension; SAbdAdd, shoulder abduction/adduction; TrunkDP, trunk displacement. (A) Shoulder flexion. (B) Pointing ahead. (C) Reach-to-grasp a glass. (D) Key insertion.

and (B), shoulder flexion/extension shows larger ranges in 4(A) and (B) when compared to $4(\mathrm{C})$ and $(\mathrm{D})$.

Spatiotemporal kinematics are illustrated in terms of shoulder-elbow angle plots for each movement task in Figure 3.5(A)-(D). Each scatter curve represents the normalized mean curve per subject arm and task. Visual exploration of the shoulder-elbow angle plots depicts that deviations in terms of an increase of elbow flexion during shoulder flexion task (1) can be observed in all subjects and both arms, while being increased in the affected upper limb in Figure 3.5(A). Shoulder-elbow angle plots of the pointing ahead movement in task (2) revealed different movement strategies to emphasize the direction to look at between subjects in both the affected and less-affected upper limb. Figure 3.5(B) illustrates, that subjects tended to either move through wide ranges of elbow flexion-extension, emphasize elbow extension at the end of the movement, or keep the elbow relatively extended throughout the movement. The shoulder-elbow angle plots of task (3) in Figure 3.5(C) illustrate comparable curve 
shapes during reaching in the affected and unaffected upper limbs. Similarly, curve shapes during task (4) in Figure 3.5(D) are comparable in both the affected upper limb. Besides the inter- and intra-individual movement variability, a preservation of the shoulder-elbow
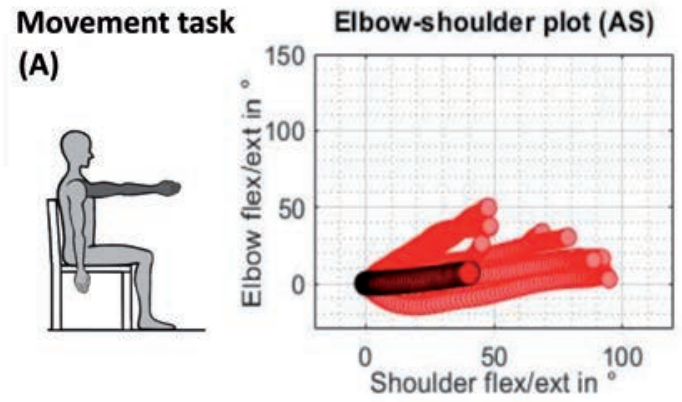

(B)
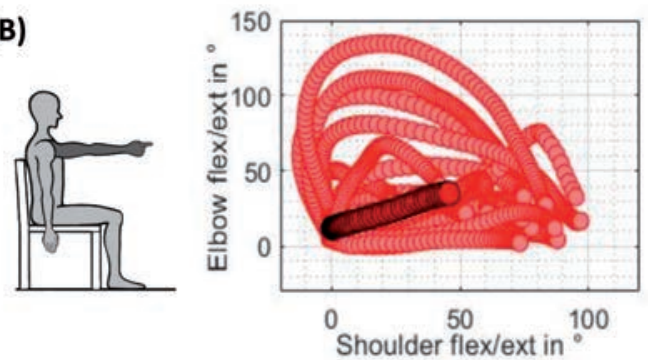

(C)
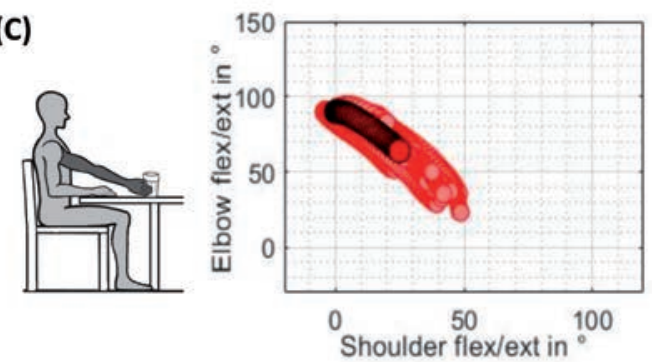

(D)
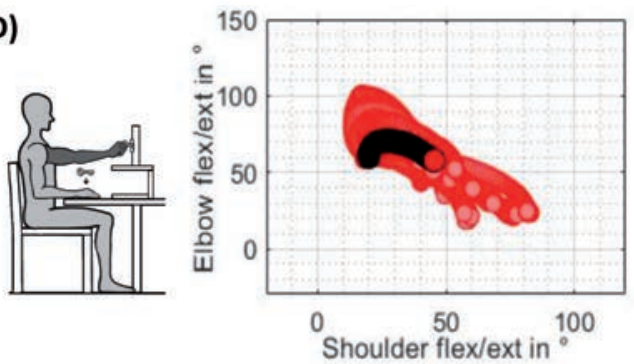
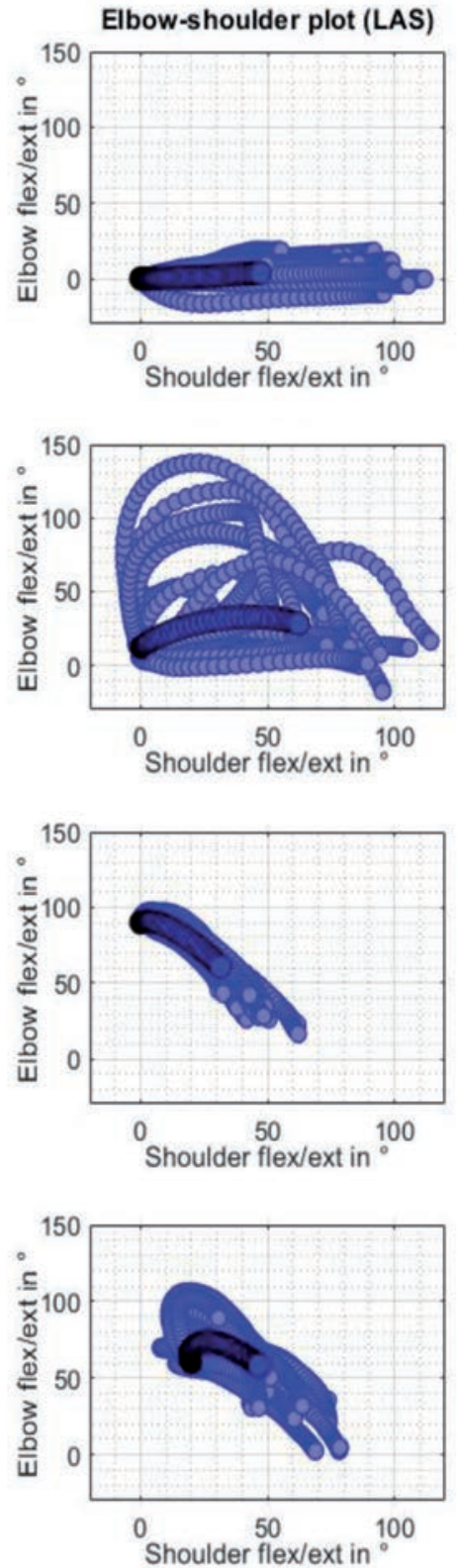

Figure 3.5. Shoulder-elbow mean curve per tested arm and task across subjects ( $N=26)$. (A) Shoulder flexion. (B) Pointing ahead. (C) Reach-to-grasp a glass. (D) Key insertion. 


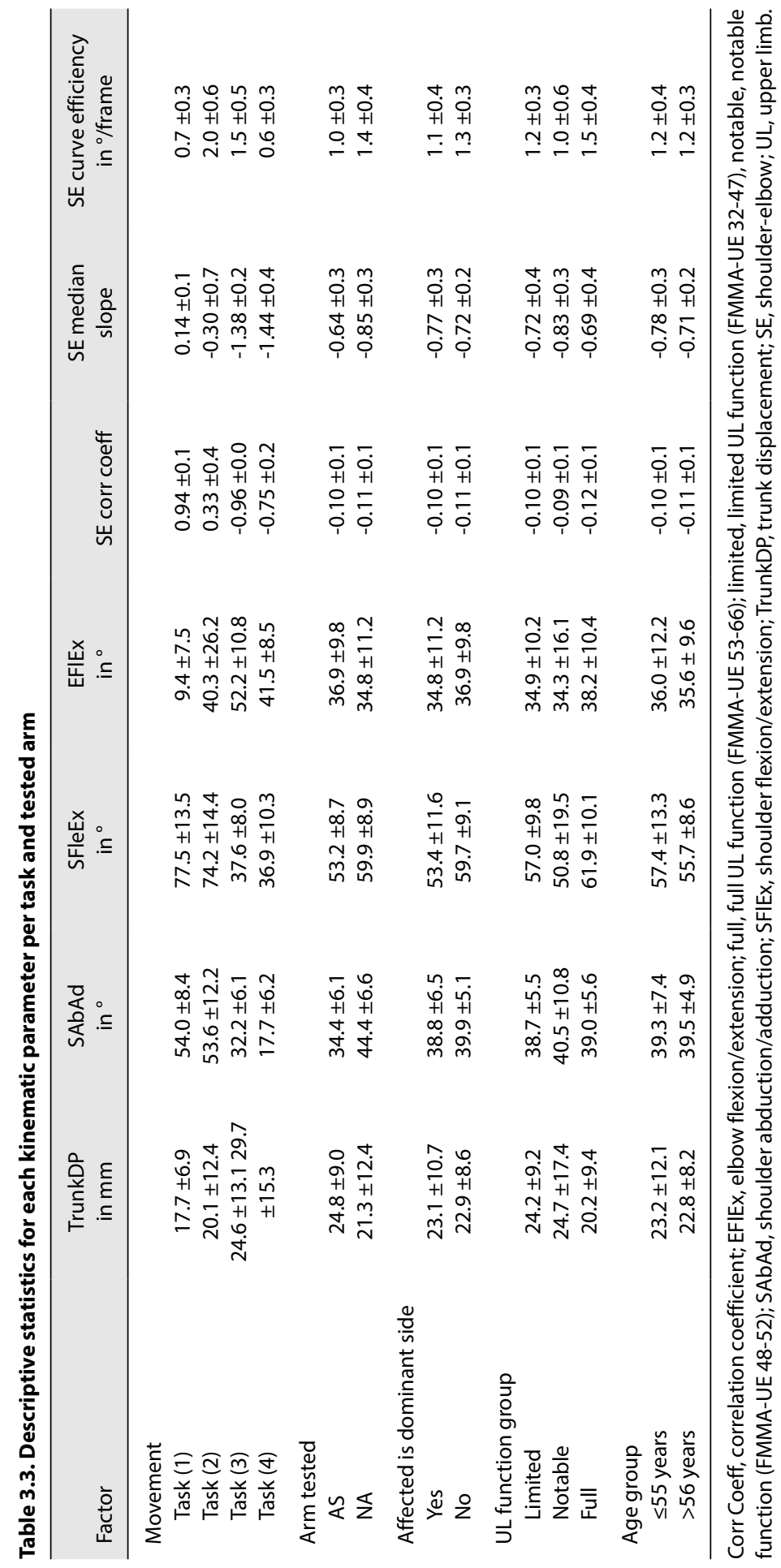


coordination can be described across the functional movement tasks, when comparing the mean curve shape per subject of the shoulder-elbow plots between the affected and the less-affected side.

\subsubsection{Effects of the factors on the spatial and spatiotemporal kinematic measures}

The mean estimates and standard deviation of the investigated kinematic parameter are presented for each fixed factor in Table 3.3. The results of the fixed effects analysis per independent factor (Task, tested arm, affected is dominant side, upper limb function, age) on each dependent kinematic measure are shown in Table 3.4. The results of post-hoc pairwise testing between the four movement tasks and the three upper limb function levels are shown in terms of p-values per kinematic parameter and factor in Table 3.4.

Statistically significant differences were found for all movement tasks and all investigated kinematic parameters as displayed in Table 3.4. Trunk displacement ranged from $1.7 \mathrm{~cm}$ to 2.9 $\mathrm{cm}$ between tasks and was only statistically significantly different between isolated shoulder

Table 3.4. Results of linear mixed model analysis

\begin{tabular}{|c|c|c|c|c|c|c|c|}
\hline \multirow[b]{2}{*}{ Factor } & \multicolumn{7}{|c|}{ Kinematic metric for interjoint coordination } \\
\hline & TrunkDP & SAbAd & SFIEx & EFIEx & $\begin{array}{l}\text { SE corr } \\
\text { coeff }\end{array}$ & $\begin{array}{l}\text { SE median } \\
\text { slope }\end{array}$ & $\begin{array}{l}\text { SE curve } \\
\text { efficiency }\end{array}$ \\
\hline Movement task & 0.001 & 0.000 & 0.000 & 0.000 & 0.000 & 0.000 & 0.000 \\
\hline Task (1) vs. Task (2) & 1.000 & 1.000 & 1.000 & 0.000 & 0.000 & 0.020 & 0.000 \\
\hline Task (1) vs. Task (3) & 0.056 & 0.000 & 0.000 & 0.000 & 0.000 & 0.000 & 0.000 \\
\hline Task (1) vs. Task (4) & 0.002 & 0.000 & 0.000 & 0.000 & 0.000 & 0.000 & 1.000 \\
\hline Task (2) vs. Task (3) & 0.861 & 0.000 & 0.000 & 0.129 & 0.000 & 0.000 & 0.001 \\
\hline Task (2) vs. Task (4) & 0.058 & 0.000 & 0.000 & 1.000 & 0.000 & 0.000 & 0.000 \\
\hline Task (3) vs. Task (4) & 0.714 & 0.000 & 1.000 & 0.000 & 0.000 & 1.000 & 0.000 \\
\hline \multicolumn{8}{|l|}{ Arm tested } \\
\hline AS vs. NA & 0.001 & 0.000 & 0.000 & 0.002 & 0.395 & 0.000 & 0.000 \\
\hline $\begin{array}{l}\text { Affected is dominant side } \\
\text { (Yes vs. No) }\end{array}$ & 0.935 & 0.413 & 0.011 & 0.251 & 0.089 & 0.161 & 0.015 \\
\hline UL function group & 0.257 & 0.693 & 0.051 & 0.264 & 0.070 & 0.069 & 0.001 \\
\hline Limited vs. notable & 1.000 & 1.000 & 0.325 & 1.000 & 0.342 & 0.122 & 0.498 \\
\hline Limited vs. full & 0.317 & 1.000 & 0.244 & 0.335 & 0.328 & 1.000 & 0.003 \\
\hline Notable vs. full & 0.791 & 1.000 & 0.053 & 0.755 & 0.073 & 0.070 & 0.005 \\
\hline \multicolumn{8}{|l|}{ Age group } \\
\hline$\leq 55$ years vs. $>56$ years & 0.889 & 0.888 & 0.540 & 0.860 & 0.125 & 0.045 & 0.394 \\
\hline
\end{tabular}

Corr coeff, correlation coefficient; EFIEx, elbow flexion/extension; full, full UL function (FMMA-UE 53-66); limited, limited UL function (FMMA-UE 32-47), notable, notable function (FMMA-UE 48-52); SAbAd, shoulder abduction/adduction; SFIEx, shoulder flexion/extension; TrunkDP, trunk displacement; SE, shoulder-elbow; UL, upper limb. Statistically significant effects are indicated in bold. 
flexion and the key insertion task $(\mathrm{F}(3,58.036)=6.119, \mathrm{p} \leq 0.001)$. Effects of the factor of the tested arm were found for all kinematic parameters except of the shoulder-elbow correlation. The factor of affected-dominant hand or affected non-dominant resulted in statistically significant effects on shoulder flexion/extension $(\mathrm{F}(1,39.832)=7.058, \mathrm{p}=0.011)$ and shoulder elbow curve efficiency $(F(1,61.565)=6.323, \mathrm{p}=0.015)$. Differences with respect to upper limb function, were detected for shoulder-elbow curve efficiency $(F(2,61.565)=7.285$, $\mathrm{p}=0.001)$ with significant differences between the limited $(\mathrm{N}=13)$ and full function $(\mathrm{N}=10)$ and between notable $(\mathrm{N}=3)$ and full function in post-hoc testing. The factor of age revealed significant effects on the dependent variable of shoulder elbow median slope with a mean of -0.784 compared to -0.705 in the less-affected side $(F(1,34.432)=4.344, p=0.045)$.

\subsubsection{Relationship between clinically measured impairment and spatiotemporal kinematics}

For the comparison between spatial and spatiotemporal kinematic measures across tasks per subject and the FMA-UE, correlation coefficients were calculated for each combination and presented in the confusion matrix in Table 3.5. The strongest statistically significant correlation with the FMA-UE was found for curve efficiency $(r=0.75)$, followed by shoulder flexion/extension ( $\mathrm{r}=0.68$ ), elbow flexion/extension $(\mathrm{r}=0.53)$ and shoulder abduction/ adduction ( $r=0.49$ ). Furthermore, strong correlations were found between elbow flexion/ extension and shoulder flexion/extension $(r=0.53)$, between elbow flexion/extension and shoulder abduction/adduction $(\mathrm{r}=0.53)$ and between shoulder flexion/extension and shoulder abduction/adduction $(r=0.57)$. For shoulder-elbow curve efficiency significant correlations were shown with shoulder flexion/extension $(\mathrm{r}=0.85)$, and elbow flexion/ extension $(\mathrm{r}=0.55)$.

The relationship between the FMMA-UE arm subsection and kinematic metrics representing measures of shoulder-elbow coordination was additionally investigated to explore the comparability of kinematic measures and shoulder-elbow coordination, as specifically tested in the FMMA-UE arm subsection. In result, a statistically significant correlation between the clinically measured impaired interjoint coordination and curve efficiency $(r=0.59$, $\mathrm{p}=0.002)$ was found. For the shoulder-elbow correlation coefficient $(r=0.24, p=0.230)$ and shoulder-elbow median slope $(r=0.09, \mathrm{p}=0.653)$ no statistically significant correlations were found with the FMMA-UE arm subsection. Figure 3.6 illustrates the subjects mean values of the correlation coefficient, the median slope and curve efficiency, over all tasks and for each task plotted against the FMMA-UE arm subsection. 
(A) Shoulder-elbow correlation coefficient per subject FMMA-UE arm subsection and task

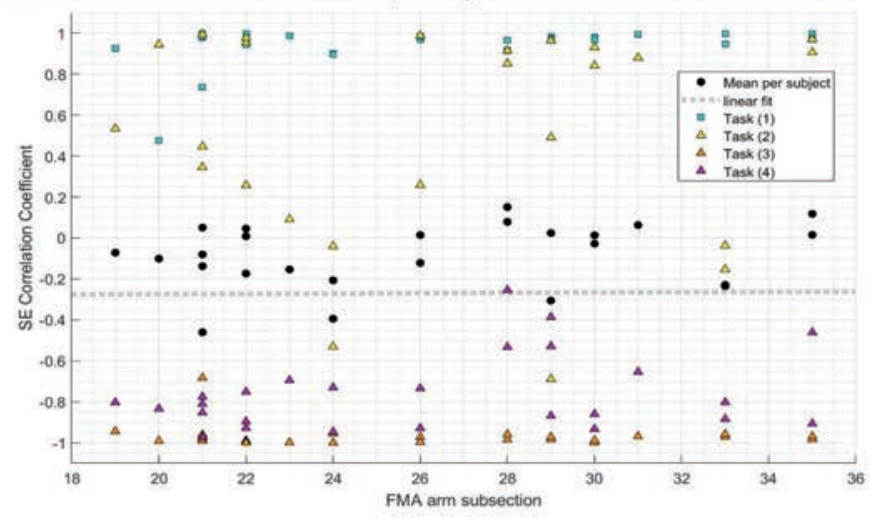

(B) Shoulder-elbow median slope per subject FMMA-UE arm subsection and task

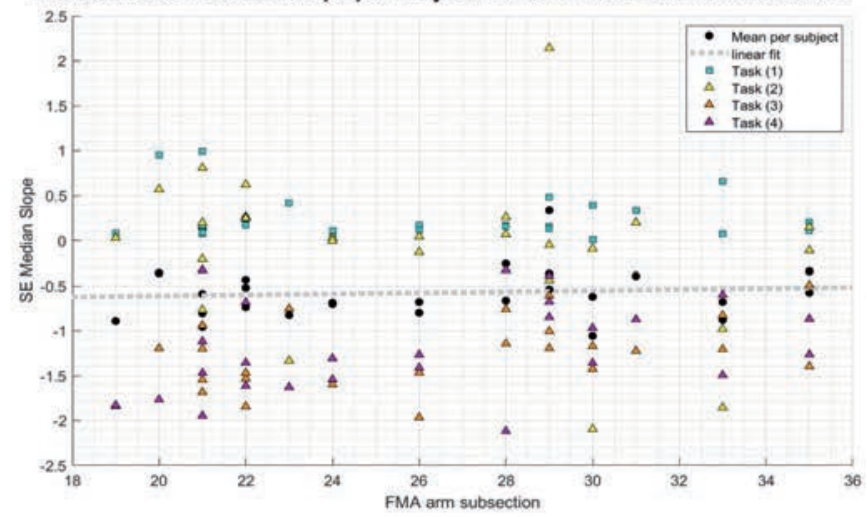

(C) Shoulder-elbow curve efficiency per subject FMMA-UE arm subsection and task

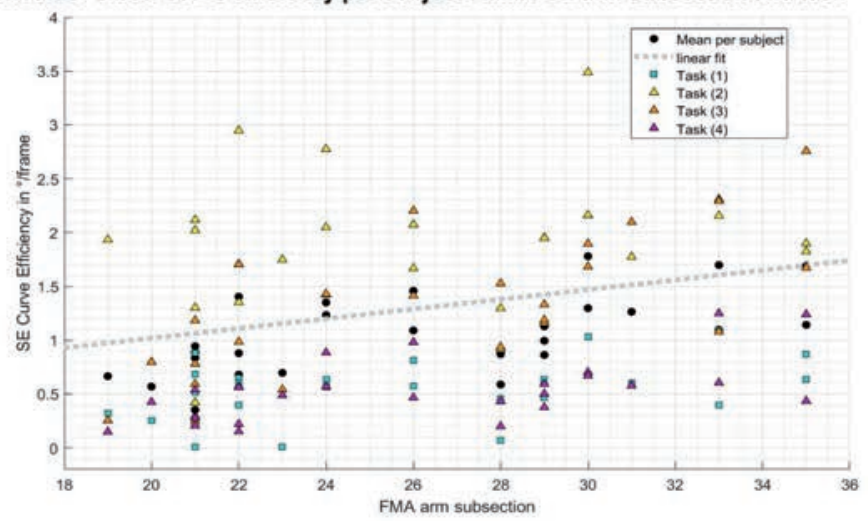

Figure 3.6. Relation between shoulder-elbow coordination metrics and the Fugl-Meyer Motor Assessment of the Upper Extremity arm subsection (19-35/36) per subject and task.

(A) Shoulder-elbow correlation coefficient per subject FMMA-UE arm subsection and task. (B) Shoulder-elbow median slope per subject FMMA-UE arm subsection and task. (C) Shoulder-elbow curve efficiency per subject FMMA-UE arm subsection and task. 
Table 3.5. Confusion matrix of correlation coefficients for each measure combination

\begin{tabular}{|c|c|c|c|c|c|c|c|}
\hline & TrunkDP & SAbAd & SFIEx & EFIEx & $\begin{array}{l}\text { SE corr } \\
\text { coeff }\end{array}$ & $\begin{array}{l}\text { SE median } \\
\text { slope }\end{array}$ & $\begin{array}{l}\text { SE curve } \\
\text { efficiency }\end{array}$ \\
\hline FMA-UE & $\begin{array}{c}-0.16 \\
p=0.436\end{array}$ & $\begin{array}{c}0.49 \\
p=0.010\end{array}$ & $\begin{array}{c}0.68 \\
p=0.000\end{array}$ & $\begin{array}{c}0.53 \\
p=0.004\end{array}$ & $\begin{array}{c}0.10 \\
p=0.603\end{array}$ & $\begin{array}{c}0.053 \\
p=0.791\end{array}$ & $\begin{array}{c}0.75 \\
p=0.000\end{array}$ \\
\hline TrunkDP & & $\begin{array}{c}-0.11 \\
p=0.595\end{array}$ & $\begin{array}{c}-0.12 \\
p=0.550\end{array}$ & $\begin{array}{c}-0.04 \\
p=0.831\end{array}$ & $\begin{array}{c}-0.10 \\
p=0.611\end{array}$ & $\begin{array}{c}-0.08 \\
p=0.678\end{array}$ & $\begin{array}{c}-0.04 \\
p=0.846\end{array}$ \\
\hline SAbAd & & & $\begin{array}{c}0.57 \\
p=0.002\end{array}$ & $\begin{array}{c}0.53 \\
p=0.004\end{array}$ & $\begin{array}{c}-0.03 \\
p=0.880\end{array}$ & $\begin{array}{c}0.28 \\
p=0.154\end{array}$ & $\begin{array}{c}0.40 \\
p=0.040\end{array}$ \\
\hline SFIEx & & & & $\begin{array}{c}0.53 \\
p=0.004\end{array}$ & $\begin{array}{c}-0.20 \\
p=0.318\end{array}$ & $\begin{array}{c}-0.02 \\
p=0.921\end{array}$ & $\begin{array}{c}0.85 \\
p=0.000\end{array}$ \\
\hline EFIEx & & & & & $\begin{array}{c}-0.26 \\
p=0.198\end{array}$ & $\begin{array}{c}-0.15 \\
p=0.450\end{array}$ & $\begin{array}{c}0.55 \\
p=0.003\end{array}$ \\
\hline SE corr coeff & & & & & & $\begin{array}{c}-0.07 \\
p=0.712\end{array}$ & $\begin{array}{c}-0.18 \\
p=0.380\end{array}$ \\
\hline $\begin{array}{l}\text { SE median } \\
\text { slope }\end{array}$ & & & & & & & $\begin{array}{c}0.01 \\
p=0.956\end{array}$ \\
\hline
\end{tabular}

EFlexExt, elbow flexion/extension; SFlexExt, shoulder flexion/extension; SAbdAdd, shoulder abduction/ adduction; Trunk DP, trunk displacement; SE, Shoulder-elbow, corr coeff, correlation coefficient; UL, upper limb. The bold measures present statically significant correlations.

\subsection{Discussion}

To our knowledge, this was the first study investigating interjoint coordination during representative upper limb tasks in chronic stroke patients with mild-to-moderate upper limb motor impairment, aiming to bridge the gap between abstract clinical motor assessments and kinematic characterization of various upper limb movements performed in daily life. Kinematic metrics reflecting interjoint coordination were investigated and compared across movement tasks by considering the covariates dominance, age and upper limb function, and related with a recommended standard clinical test, the FMMA-UE (Kwakkel et al., 2017; Kwakkel et al., 2019; Burridge et al., 2019). It was found that the values of kinematic metrics were largely dependent on the movement task and the tested arm, while age and the affected dominant side hardly influenced the metrics. The fact that both spatial and spatiotemporal metrics of the shoulder-elbow complex were largely dependent on the movement performed, underpins the need to assess upper limb interjoint coordination in different task contexts. Interestingly, the elbow joint ranges were significantly different and less variable during isolated shoulder flexion task $\left(9.4^{\circ} \pm 7.5^{\circ}\right)$, representative for one of the FMMA-UE items, when compared to the pointing task $\left(40.3^{\circ} \pm 26.2^{\circ}\right)$, even though both tasks shared the same 
person-related workspace and target position, indicating the differences of the FMMA-UE from natural movement behavior. Comparing results of the clinical measured impairment with the FMMA-UE and results on kinematic metrics across all tasks, revealed moderate correlations between the FMMA-UE or FMMA-UE arm subsection and metrics on shoulder and elbow joint ranges, and shoulder-elbow curve efficiency ( $r \geq 0.5)$, besides low correlations between trunk metrics and shoulder-elbow correlation coefficient and median slope.

All spatial and spatiotemporal kinematic measures except the shoulder-elbow correlation coefficient showed statistically significant discriminability between pathological movement behavior of the affected upper limb and physiological movement behavior of the less-affected upper limb.

Trunk motions ranged between a mean of 1.8 and $3.0 \mathrm{~cm}$, tending to increase from the shoulder flexion, pointing ahead, reach-to-grasp a glass, to the key insertion task. This illustrates an increased trunk compensation with an increase in task complexity by requiring distal upper limb interactions with objects (McIsaac et al., 2015). Trunk compensatory movements were shown to be slightly but significantly increased when moving the affected limb (mean of $2.5 \mathrm{~cm}$ ) when compared to the less-affected upper limb (mean of $2.1 \mathrm{~cm}$ ). However, these differences were small, when compared to previous findings of trunk movements of around $10 \mathrm{~cm}$ in stroke subjects during reach-to-point (Cirstea et al., 2000) and reach-to-grasp (Alt Murphy et al., 2018). Hence, the presented results fall within the limits of 2 to $5 \mathrm{~cm}$ as a clinically meaningful cut-off score for compensatory trunk movements (Alt Murphy et al., 2013). The differences in the shoulder DOF can be partially explained by differences in target height between tasks, especially between the reach-to-grasp a glass on the table that requires less shoulder flexion when compared to the other movement tasks with targets on shoulder-height-level. Shoulder joint ranges in flexion/extension and abduction/adduction were diminished in the affected arm in comparison to the lessaffected arm with joint ranges $53^{\circ}$ vs. $60^{\circ}$ and $34^{\circ}$ vs. $44^{\circ}$ respectively, suggesting inefficient activation or weakness of the shoulder muscles and the inability to cope with antigravity torques (Roh et al., 2013). Elbow flexion/extension ranged from a mean of $9.4^{\circ}$ in isolated shoulder flexion and around $52^{\circ}$ during functional task execution. The larger ranges in elbow flexion/extension during functional movements when compared to non-functional isolated shoulder flexion support the idea, that the elbow joint is rather dynamically involved in reaching movements of daily life activities than being involved as a stable or stabilizing component of a movement as predominantly examined in the FMMA-UE. 
On the level of spatiotemporal measures of shoulder-elbow coordination, values of the correlation coefficient largely varied between $r=-0.9$ and $r=0.9$ within and between subjects with tight connection to the movement tasks as illustrated in Figure 3.4. The correlation coefficient is a measure of the linear relationship between two variables, such as shoulder flexion/extension and elbow flexion/extension. Although the correlation coefficient provides estimates of general trend between two variables, it does not consider non-linearity in rather bell-shaped angle-angle curves. The shoulder-elbow median slope represents estimates of the relationship between two DOF per timeframe (Banina et al., 2017). Both the correlation coefficient and the median slope are quantifications of the overall trend in the shoulderelbow curve and depend on both, the type of movement, as well as, whether the shoulder and elbow move inphase or outphase. Consequentially, both metrics are limited to the general relationship between two joints. Shoulder elbow curve efficiency ranged between a mean of 0.14 and $1.44^{\circ}$ /frame with respect to the movement task. Curve efficiency was considerably lower in the shoulder flexion and key insertion task, when compared to the other tasks, which could be an indicator of the increased requirements on movement preciseness during key insertion and increased internal attentional focus during isolated shoulder flexion. Curve efficiency was introduced as a novel measure of interjoint coordination that combines the absolute spatial changes in two DOF, while considering temporal aspects in terms of timeframes needed to perform the movement. In that sense, curve efficiency accounts to the proposed definition of interjoint coordination by Tomita and coworkers as "a goaloriented process in which the DOF are organized in both spatial and temporal domains such that the body configuration enables the endpoint to reach to a desired location in a context dependent manner" (Tomita et al., 2017). Herein, curve efficiency has proven to be discriminable with respect to the factor whether the affected hand is the dominant hand and with respect to the upper limb motor function group, indicating promising associations with upper limb motor impairment levels.

Taken together, these findings confirm the importance of including different upper limb movement tasks when looking at interjoint coordination in patients after stroke and nondisabled adults, as the task strongly affect kinematic metric outcomes (Jeannerod et al., 1999; Michaelsen et al., 2004; Mesquita et al., 2020). Adding up to these task-related kinematic differences, research on functional brain activation provides evidence that cerebral control of upper limb movements is arranged in a task-specific action topography by taking the activity as a whole, rather than being controlled by separating or combining movement components or specific or fixed brain areas (Handjaras et al., 2015; Leo et al., 
2016). The findings of the present study emphasize the importance to consider the effects of the movement purpose, the attentional focus, and the movement complexity on kinematic expressions complementary to clinical assessment evaluations. Unlike the shoulder flexion movement of the FMMA-UE that relies on an internal movement focus and a stable extended elbow position, the three representative functional tasks rely on an external movement focus with mainly inverse kinematics between shoulder flexion and elbow extension and bell-shaped angle-angle profiles. Even though further curve fitting analysis was out of the scope of the present study, visual inspection of the shoulder-elbow angle plots suggests that motions in the shoulder and elbow were diminished in the affected side when compared to the less-affected side while the task-associated shapes seem to be largely preserved in the affected limb. Theses results furthermore underpin the challenge to clearly distinguish pathological from physiological interjoint coordination and movement activation in terms of active range of motion and strength, at least in natural surroundings including the constant influence of gravity.

The relationship between the spatiotemporal kinematic measures and the clinically measured upper limb motor impairment was explored as a part of validity. The herein presented findings on correlation between the FMMA-UE and spatial metrics shoulder flexion/ extension and elbow flexion/extension are in line with research on validity (Rech et al., 2019; van Kordelaar et al., 2012; de Paiva Silva et al., 2014; Massie et al., 2011; Massie et al., 2014; Finley et al., 2012; Li et al., 2015). In contrast to existing research (van Kordelaar et al., 2012; Massie et al., 2014; Subramanian et al., 2010; de Paiva Silva et al., 2014; Finley et al., 2012), we did not find a strong correlation between trunk displacement during various tasks and the FMMA-UE total score. The strong correlation between curve efficiency and shoulder flexion/extension and elbow flexion/extension found in this study, might be related to the fact that curve efficiency is a derivative of both DOF besides the temporal aspect of this movement parameter. The fact that shoulder-elbow curve efficiency significantly correlated with the FMMA-UE arm subsection supports the idea that it measures the same construct of interjoint coordination in the upper extremity. Future work on upper limb kinematic measurements after stroke should investigate its clinimetric properties, such as reliability, measurement error, and responsiveness.

\subsubsection{Limitations}

The spatiotemporal kinematic analysis of this study was limited to three out of seven DOFs, namely shoulder flexion/extension, shoulder abduction/adduction and elbow flexion/ 
extension, even though rotational movements and the forearm and hand component are known to be part of movement quality. We decided to examine interjoint coordination on the basic level of the two joints that contribute most to the movement performance and present characteristics stroke-related movement phenotypes, such as the pathological flexor synergy. Another limitation relates to the fact that we have considered the less-affected upper limb as the physiological movement comparator, even though we were aware of the evidence on movement limitations in the ipsilesional upper limb (Bustren et al., 2016). Nevertheless, the less-affected upper limb represents a valuable comparator in the asymmetrical impairment of unilateral stroke and is always available to the patient and the assessor in clinical practice (Lang et al., 2017). For this reason, comparisons between the affected and the less-affected upper limb remain the best-available comparator in terms of movement quality measures until a reasonable amount of normative kinematic data from the healthy population is available.

We have not controlled for possible strength limitations and therefore were not able to differentiate between weakness and interjoint coordination in the presented experimental set-up, as gradually studied by Dewald and colleagues (Sukal et al., 2007; Ellis et al., 2016). This could be induced by including gradual armload increase during movement task execution. Apart from that, real-world upper limb functions are performed not only in sitting but also in other body positions, such as standing. The fact that the pioneering works on interjoint coordination and synergistic control after stroke emphasized the influence of the postural setting of the subject on synergistic control (Fugl-Meyer et al., 1975) supports further research on this topic and its consideration in upper limb assessments.

In the current study, a wearable inertial sensing suit was used and this goes against recent recommendations to capture upper limb kinematics by an opto-electronic device (Kwakkel et al., 2019). However, the pros of wearable sensing suits are the wide applicability in flexible environments, the avoidance of problems with marker-occlusion during object manipulation, and the comparably less time-consuming system set-up (pre- and post-processing) and costs of the equipment (Warmsley et al., 2018). Based on previous research, the reliability and measurement error has shown to be comparable between inertial sensing and optoelectronic system (Robert-Lachaine et al., 2017), even when the system was used by an unexperienced person (Al-Amri et al., 2020).

Lastly, it needs to be acknowledged that other analytical approaches on the kinematic data, such as dimension-reduction approaches, would have been possible, allowing presenting 
other kinematic outcomes (Schwarz and Averta et al., 2019). Kinematic measures of the movement smoothness domain have been used for quantifying interjoint coordination based on accelerometer or gyroscope signals in the lower limb during gait assessment (Beck et al., 2018) and should additionally considered in future work of upper limb interjoint coordination besides the herein proposed measures.

\subsubsection{Future research}

Future research should expand on an upper limb movement task set (Kwakkel et al., 2019) allowing to assess the widest possible range of the tested subjects' functional capabilities by considering a stepwise increase of movement task complexity, task instruction and focus (McIsaac et al., 2015). Including a functional planar task on the table-level, such as wiping or shape-drawing, besides gesture movements, reach-to-grasp and manipulating activities should be considered in such a task set and future works to enable kinematic evaluations in stroke-subject with lower levels of upper limb function and reducing load on the shoulder. Dual-task conditions should be included in the highest level of task difficulty to assess the functional capability under real-world conditions, for example when cooking and talking at the same time, as well as to uncover subliminal deficits that still might impact the persons performance level in daily life. Another important aspect in upper limb assessments reflecting needs of real-world use is the consideration of posture. In this line, it would be interesting to investigate the impact of posture on upper limb kinematics. The resemblance with daily life tasks in such an assessment protocol is likely to ease task understanding and naturalness of the performance even in subjects with difficulties in understanding.

\subsection{Conclusion}

The presented work on qualitative upper limb movement analysis confirmed that kinematic measures of interjoint coordination in the shoulder-elbow-trunk complex are largely depending on the movement task and the tested arm in chronic stroke patients with mild-to-moderate upper limb motor impairments. The kinematic metrics during functional movements showed different expressions and variability when compared to those of the non-functional isolated shoulder-flexion, supporting the importance to assess different movement tasks in order to get a more complete picture of the patient's quality of movement. The metrics correlate at the best moderately with standard clinical tests, which underlines their benefit. Among the investigated spatiotemporal measures of shoulder-elbow 
coordination, curve efficiency showed promising discriminability between the affected and the less-affected side, the factor of affected hand dominance, and upper limb functionality and correlated well with the FMMA-UE and the FMMA-UE arm subsection respectively. Consequentially, this study contributes to novel approaches in post-stroke upper limb assessment methodologies by combining technological opportunities to measure aspects of body function during activities that are close to real world and representative for the ICF activities and participation domain.

\subsection{References}

Al-Amri M, Nicholas K, Button K, Sparkes V, Sheeran L, Davies JL. Inertial Measurement Units for Clinical Movement Analysis: Reliability and Concurrent Validity. Sensors (Basel). 2018;18:719. doi: $10.3390 / \mathrm{s} 18030719$

Allison R, Shenton L, Bamforth K, Kilbride C, Richards D. Incidence, Time Course and Predictors of Impairments Relating to Caring for the Profoundly Affected arm After Stroke: A Systematic Review. Physiother Res Int. 2016;21:210-27. doi: 10.1002/pri.1634

Alt Murphy M, Murphy S, Persson HC, Bergström UB, Sunnerhagen KS. Kinematic Analysis Using 3D Motion Capture of Drinking Task in People With and Without Upper-extremity Impairments. J Vis Exp. 2018;133:e57228. doi: 10.3791/57228

Alt Murphy M, Willén C, Sunnerhagen KS. Responsiveness of upper extremity kinematic measures and clinical improvement during the first three months after stroke. Neurorehabil Neural Repair. 2013;27:844-53. doi: 10.1177/1545968313491008

Alt Murphy M, Willen C, Sunnerhagen KS. Kinematic variables quantifying upper-extremity performance after stroke during reaching and drinking from a glass. Neurorehabil Neural Repair. 2011;25:71-80.

Baniña MC, Mullick AA, McFadyen BJ, Levin MF. Upper limb obstacle avoidance behavior in individuals with stroke. Neurorehabil Neural Repair. 2017;31:133-46.

Beck Y, Herman T, Brozgol M, Giladi N, Mirelman A, Hausdorff JM. SPARC: a new approach to quantifying gait smoothness in patients with Parkinson's disease. J Neuroeng Rehabil. 2018;15:49. doi: 10.1186/s12984-018-0398-3

Beer RF, Ellis MD, Holubar BG, Dewald JP. Impact of gravity loading on post-stroke reaching and its relationship to weakness. Muscle Nerve. 2007;36:242-50.

Bernhardt J, Hayward KS, Kwakkel G, et al. Agreed definitions and a shared vision for new standards in stroke recovery research: The Stroke Recovery and Rehabilitation Roundtable taskforce. Int J Stroke. 2017;12:444-50. doi: 10.1177/1747493017711816

Bohannon RW, Smith MB. Interrater reliability of a modified Ashworth scale of muscle spasticity. Phys Ther. 1987;67:206-7. doi: 10.1093/ptj/67.2.206

Brunnstrom S. Motor testing procedures in hemiplegia: based on sequential recovery stages. Phys Ther. 1966;46:357-75. doi: 10.1093/ptj/46.4.357

Brunnstrom S. Movement Therapy in Hemiplegia: A Neurophysiological Approach. Medical Dept, Harper \& Row, New York; 1970. 
Bustrén EL, Sunnerhagen KS, Alt Murphy M. Movement Kinematics of the Ipsilesional Upper Extremity in Persons With Moderate or Mild Stroke. Neurorehabil Neural Repair. 2017;31:376-86. doi: $10.1177 / 1545968316688798$

Cirstea MC, Levin MF. Improvement of arm movement patterns and endpoint control depends on type of feedback during practice in stroke survivors. Neurorehabil Neural Repair. 2007;21:398-411.

Cirstea MC, Mitnitski AB, Feldman AG, Levin MF. Interjoint coordination dynamics during reaching in stroke. Exp Brain Res. 2003;151:289-300. doi: 10.1007/s00221-003-1438-0

Corbetta D, Sirtori V, Castellini G, Moja L, Gatti R. Constraint-induced movement therapy for upper extremities in people with stroke. Cochrane Database Syst Rev. 2015;2015:CD004433. doi: 10.1002/14651858.CD004433.pub3

de Paiva Silva FP, Freitas SM, Silva PV, Banjai RM, Alouche SR. Ipsilesional arm motor sequence performance after right and left hemisphere damage. J Mot Behav. 2014;46:407-14.

Dong Y, Yean Lee W, Hilal S, et al. Comparison of the Montreal Cognitive Assessment and the MiniMental State Examination in detecting multi-domain mild cognitive impairment in a Chinese sub-sample drawn from a population-based study. Int Psychogeriatr. 2013;25:1831-8. doi: $10.1017 /$ S1041610213001129

Ellis MD, Carmona C, Drogos J, Traxel S, Dewald JP. Progressive abduction loading therapy targeting flexion synergy to regain reaching function in chronic stroke: Preliminary results from an RCT. Ann Int Con IEEE Eng Med Biol Soc. 2016;2016:5837-40. doi: 10.1109/EMBC.2016.7592055

Eraifej J, Clark W, France B, Desando S, Moore D. Effectiveness of upper limb functional electrical stimulation after stroke for the improvement of activities of daily living and motor function: a systematic review and meta-analysis. Syst Rev. 2017;6:40.

Feix T, Romero J, Schmiedmayer H, Dollar AM, Kragic D. The GRASP Taxonomy of Human Grasp Types. IEEE Trans Hum Mach Syst. 2016;46:1-12. doi: 10.1109/THMS.2015.2470657.

Finley M, Combs S, Carnahan K, Peacock S, Buskirk AV. Comparison of 'less affected limb' reaching kinematics in individuals with chronic stroke and healthy age-matched controls. Phys Occup Ther Geriatr. 2012;30:245-59.

Fugl-Meyer AR, Jääskö L, Leyman I, Olsson S, Steglind S. The post-stroke hemiplegic patient. 1. a method for evaluation of physical performance. Scand J Rehabil Med. 1975;7:13.

Gladstone DJ, Danells CJ, Black SE. The fugl-meyer assessment of motor recovery after stroke: a critical review of its measurement properties. Neurorehabil Neural Repair. 2002;16:232-40. doi: $10.1177 / 154596802401105171$

Hoonhorst MH, Nijland RH, van den Berg JS, Emmelot CH, Kollen BJ, Kwakkel G. How Do FuglMeyer Arm Motor Scores Relate to Dexterity According to the Action Research Arm Test at 6 Months Poststroke? Arch Phys Med Rehabil. 2015;96:1845-9. doi: 10.1016/j.apmr.2015.06.009

Houwink A, Steenbergen B, Prange GB, Buurke JH, Geurts AC. Upper-limb motor control in patients after stroke: Attentional demands and the potential beneficial effects of arm support. Hum Mov Sci. 2013;32:377-87.

Jeannerod M. The neural and behavioral organization of goal-directed movements. Oxford: Clarendon Press; 1990.

Jørgensen HS, Nakayama H, Raaschou HO, Olsen TS. Neurologic and functional recovery the Copenhagen Stroke Study. Phys Med Rehabil Clin N Am. 1999;10:887-906.

Krabben T, Molier BI, Houwink A, Rietman JS, Buurke JH, Prange GB. Circle drawing as evaluative movement task in stroke rehabilitation: An explorative study. J Neuroeng Rehabil. 2011;8:15. 
Krakauer JW, Carmichael ST. Broken Movement. Neurobiology of Motor Recovery after Stroke. MIT Press; 2017.

Kwakkel G, Lannin NA, Borschmann K, et al. Standardized measurement of sensorimotor recovery in stroke trials: Consensus-based core recommendations from the Stroke Recovery and Rehabilitation Roundtable. Int J Stroke. 2017;12:451-61. doi: 10.1177/1747493017711813

Kwakkel G, van Wegen EEH, Burridge JH, et al. Standardized Measurement of Quality of Upper Limb Movement After Stroke: Consensus-Based Core Recommendations From the Second Stroke Recovery and Rehabilitation Roundtable. Neurorehabil Neural Repair. 2019;33:951-8. doi: $10.1177 / 1545968319886477$

Lang CE, Waddell KJ, Klaesner JW, Bland MD. A Method for Quantifying Upper Limb Performance in Daily Life Using Accelerometers. J Vis Exp. 2017;122:55673. doi: 10.3791/55673

Leo A, Handjaras G, Bianchi M, et al. A synergy-based hand control is encoded in human motor cortical areas. Elife. 2016;15:e13420. doi: 10.7554/eLife.13420

Levin MF. Interjoint coordination during pointing movements is disrupted in spastic hemiparesis. Brain. 1996;119(Pt 1):281-93. doi: 10.1093/brain/119.1.281

Levin MF, Kleim JA, Wolf SL. What do motor "recovery" and "compensation" mean in patients following stroke? Neurorehabil Neural Repair. 2009;23:313-9. doi: 10.1177/1545968308328727

Levin MF, Liebermann DG, Parmet Y, Berman S. Compensatory versus noncompensatory shoulder movements used for reaching in stroke. Neurorehabil Neural Repair. 2016;30:635-46.

Li KY, Lin KC, Chen CK, Liing RJ, Wu CY, Chang WY. Concurrent and predictive validity of arm kinematics with and without a trunk restraint during a reaching task in individuals with stroke. Arch Phys Med Rehabil. 2015;96:1666-75.

Massie CL, Fritz S, Malcolm MP. Elbow extension predicts motor impairment and performance after stroke. Rehabil Res Pract. 2011;2011:381978.

Massie C, Malcolm M, Greene D, Browning R. Biomechanical contributions of the trunk and upper extremity in discrete versus cyclic reaching in survivors of stroke. Top Stroke Rehabil. 2014;21: 23-32.

McIsaac TL, Lamberg EM, Muratori LM. Building a framework for a dual task taxonomy. Biomed Res Int. 2015;2015:591475. doi: 10.1155/2015/591475

McMorland AJ, Runnalls KD, Byblow WD. A neuroanatomical framework for upper limb synergies after stroke. Front Hum Neurosci. 2015;9:82. doi: 10.3389/fnhum.2015.00082

Mesquita IA, Fonseca PFPD, Borgonovo-Santos M, Ribeiro E, Pinheiro ARV, Correia MV, Silva C. Comparison of upper limb kinematics in two activities of daily living with different handling requirements. Hum Mov Sci. 2020;72:102632. doi: 10.1016/j.humov.2020.102632

Michaelsen SM, Jacobs S, Roby-Brami A, Levin MF. Compensation for distal impairments of grasping in adults with hemiparesis. Exp Brain Res. 2004;157:162-73. doi: 10.1007/s00221-004-1829-x

Persson HC, Parziali M, Danielsson A, Sunnerhagen KS. Outcome and upper extremity function within 72 hours after first occasion of stroke in an unselected population at a stroke unit. A part of the SALGOT study. BMC Neurol. 2012;12:162.

Prinsen CAC, Mokkink LB, Bouter LM, Alonso J, Patrick DL, de Vet HCW, Terwee CB. COSMIN guideline for systematic reviews of patient-reported outcome measures. Qual Life Res. 2018;27: 1147-57. doi: 10.1007/s11136-018-1798-3

Raghavan P. Upper Limb Motor Impairment After Stroke. Phys Med Rehabil Clin N Am. 2015;26:599610. doi: 10.1016/j.pmr.2015.06.008 
Rech KD, Salazar AP, Marchese RR, Schifino G, Cimolin V, Pagnussat AS. Fugl-Meyer Assessment Scores Are Related With Kinematic Measures in People with Chronic Hemiparesis after Stroke. J Stroke Cerebrovasc Dis. 2020;29:104463. doi: 10.1016/j.jstrokecerebrovasdis.2019.104463

Robert-Lachaine X, Mecheri H, Larue C, Plamondon A. Accuracy and repeatability of single-pose calibration of inertial measurement units for whole-body motion analysis. Gait Posture. 2017;54:80-6. doi: 10.1016/j.gaitpost.2017.02.029

Robert-Lachaine X, Mecheri H, Larue C, Plamondon A. Validation of inertial measurement units with an optoelectronic system for whole-body motion analysis. Med Biol Eng Comput. 2017;55:60919. doi: 10.1007/s11517-016-1537-2

Roetenberg D, Slycke PJ, Veltink PH. Ambulatory position and orientation tracking fusing magnetic and inertial sensing. IEEE Trans Biomed Eng. 2007a;54:883-90. doi: 10.1109/TBME.2006.889184

Roetenberg D, Baten CT, Veltink PH. Estimating body segment orientation by applying inertial and magnetic sensing near ferromagnetic materials. IEEE Trans Neural Syst Rehabil Eng. 2007b; 15:469-71. doi: 10.1109/TNSRE.2007.903946

Roh J, Rymer WZ, Perreault EJ, Yoo SB, Beer RF. Alterations in upper limb muscle synergy structure in chronic stroke survivors. J Neurophysiol. 2013;109:768-81. doi: 10.1152/jn.00670.2012

Santello M, Lang CE. Are movement disorders and sensorimotor injuries pathologic synergies? When normal multi-joint movement synergies become pathologic. Front Hum Neurosci. 2015;8:1050. doi: 10.3389/fnhum.2014.01050

Santisteban L, Térémetz M, Bleton JP, Baron JC, Maier MA, Lindberg PG. Upper Limb Outcome Measures Used in Stroke Rehabilitation Studies: A Systematic Literature Review. PLoS One. 2016;11:e0154792. doi: 10.1371/journal.pone.0154792

Schambra HM, Parnandi A, Pandit NG, Uddin J, Wirtanen A, Nilsen DM. A Taxonomy of Functional Upper Extremity Motion. Front Neurol. 2019;10:857. doi: 10.3389/fneur.2019.00857

Schwarz A, Kanzler CM, Lambercy O, Luft AR, Veerbeek JM. Systematic Review on Kinematic Assessments of Upper Limb Movements After Stroke. Stroke. 2019;50:718-27. doi: 10.1161/ STROKEAHA.118.023531

Sethi A, Stergiou N, Patterson TS, Patten C, Richards LG. Speed and rhythm affect temporal structure of variability in reaching poststroke: A pilot study. J Mot Behav. 2017;49:35-45.

Stolk-Hornsveld F, Crow JL, Hendriks EP, van der Baan R, Harmeling-van der Wel BC. The Erasmus MC modifications to the (revised) Nottingham Sensory Assessment: a reliable somatosensory assessment measure for patients with intracranial disorders. Clin Rehabil. 2006;20:160-72. doi: $10.1191 / 0269215506 \mathrm{cr} 932 \mathrm{oa}$

Subramanian SK, Yamanaka J, Chilingaryan G, Levin MF. Validity of movement pattern kinematics as measures of arm motor impairment poststroke. Stroke. 2010;41:2303-8.

Sukal TM, Ellis MD, Dewald JP. Shoulder abduction-induced reductions in reaching work area following hemiparetic stroke: neuroscientific implications. Exp Brain Res. 2007;183:215-23. doi: 10.1007/s00221-007-1029-6

Taub E, Uswatte G, Mark VW, Morris DM. The learned nonuse phenomenon: implications for rehabilitation. Eura Medicophys. 2006;42:241-56.

Thrane G, Sunnerhagen KS, Persson HC, Opheim A, Alt Murphy M. Kinematic upper extremity performance in people with near or fully recovered sensorimotor function after stroke. Physiother Theory Pract. 2019;35:822-32. doi: 10.1080/09593985.2018.1458929 
Tomita Y, Rodrigues MRM, Levin MF. Upper Limb Coordination in Individuals With Stroke: Poorly Defined and Poorly Quantified. Neurorehabil Neural Repair. 2017;31:885-97. doi: 10.1177/ 1545968317739998

Twitchell TE. The restoration of motor function following hemiplegia in man. Brain. 1951;74:443-80. doi: 10.1093/brain/74.4.443

van Kordelaar J, van Wegen EE, Kwakkel G. Unraveling the interaction between pathological upper limb synergies and compensatory trunk movements during reach-to-grasp after stroke: A crosssectional study. Exp Brain Res. 2012;221:251-62.

van Kordelaar J, van Wegen EE, Nijland RH, Daffertshofer A, Kwakkel G. Understanding adaptive motor control of the paretic upper limb early poststroke: The explicit-stroke program. Neurorehabil Neural Repair. 2013;27:854-63.

van Meulen FB, Klaassen B, Held J, Reenalda J, Buurke JH, van Beijnum BF, Luft A, Veltink PH. Objective Evaluation of the Quality of Movement in Daily Life after Stroke. Front Bioeng Biotechnol. 2016;3:210. doi: 10.3389/fbioe.2015.00210

van Meulen FB, Reenalda J, Buurke JH, Veltink PH. Assessment of daily-life reaching performance after stroke. Ann Biomed Eng. 2015;43:478-86.

Veerbeek JM, Langbroek-Amersfoort AC, van Wegen EE, Meskers CG, Kwakkel G. Effects of RobotAssisted Therapy for the Upper Limb After Stroke. Neurorehabil Neural Repair. 2017;31:107-21. doi: $10.1177 / 1545968316666957$

Walmsley CP, Williams SA, Grisbrook T, Elliott C, Imms C, Campbell A. Measurement of Upper Limb Range of Motion Using Wearable Sensors: A Systematic Review. Sports Med Open. 2018;4:53. doi: 10.1186/s40798-018-0167-7.

Woodbury ML, Howland DR, McGuirk TE, et al. Effects of trunk restraint combined with intensive task practice on poststroke upper extremity reach and function: A pilot study. Neurorehabil Neural Repair. 2009;23:78-91.

Wu C-Y, Liing R-J, Chen H-C, Chen C-L, Lin K-C. Arm and trunk movement kinematics during seated reaching within and beyond arm's length in people with stroke: A validity study. Phys Ther. 2014;94:845-56.

Wu G, van der Helm FC, Veeger HE, et al. ISB recommendation on definitions of joint coordinate systems of various joints for the reporting of human joint motion--Part II: shoulder, elbow, wrist and hand. J Biomech. 2005;38:981-92. doi: 10.1016/j.jbiomech.2004.05.042

Xsene MVN User Manual, User Guide Xsens MVN, MVN Link, MVN Awinda, Document MV0319P, Revision X, Oct 2018. Xsens: Enschede, the Netherlands. Accessed on November 2018.

Yang Q, Yang Y, Luo J, Li L, Yan T, Song R. Kinematic outcome measures using target-reaching arm movement in stroke. Ann Biomed Eng. 2017;45:2794-803.

Yew KS, Cheng E. Acute stroke diagnosis. Am Fam Physician. 2009;80:33-40. 



\title{
A functional analysis-based approach to quantify upper limb impairment level in chronic stroke patients: a pilot study
}

\author{
A. Schwarz \\ G. Averta \\ J.M. Veerbeek \\ A.R. Luft \\ J.P.O. Held \\ G. Valenza \\ A. Bicchi \\ M. Bianchi
}




\subsection{Abstract}

The accurate assessment of upper limb motion impairment induced by stroke - which represents one of the primary causes of disability world-wide - is the first step to successfully monitor and guide patients' recovery. As of today, the majority of the procedures relies on clinical scales, which are mostly based on ordinal scaling, operator-dependent, and subject to floor and ceiling effects. In this work, we intend to overcome these limitations by proposing a novel approach to analytically evaluate the level of pathological movement coupling, based on the quantification of movement complexity. To this goal, we consider the variations of functional Principal Components applied to the reconstruction of joint angle trajectories of the upper limb during daily living task execution, and compared these variations between two conditions, i.e. the affected and non-affected arm. A Dissimilarity Index, which codifies the severity of the upper limb motor impairment with respect to the movement complexity of the non-affected arm, is then proposed. This methodology was validated as a proof of concept upon a set of four chronic stroke subjects with mild to moderate arm and hand impairments. As a first step, we evaluated whether the derived outcomes differentiate between the two conditions upon the whole data set. Secondly, we exploited this concept to discern between different subjects and impairment levels. Results show that: i) differences in terms of movement variability between the affected and nonaffected upper limb are detectable and ii) different impairment profiles can be characterized for single subjects using the proposed approach. Although provisional, these results are very promising and suggest this approach as a basis ingredient for the definition of a novel, operator-independent, sensitive, intuitive and widely applicable scale for the evaluation of upper limb motion impairment. 


\subsection{Introduction}

Human upper limb movements require an extraordinary coordination of numerous degrees of freedom (DoFs). Based on neuroscientific literature, this coordination is supposed to be organized according to covariation patterns - the so-called synergistic control, which allow a successful interaction with the environment (Santello, 2014; Tresch and Jarc, 2009; Della Santina et al., 2017; Latash, 2008). After stroke, these upper limb movements can be affected by different types of sensorimotor impairments, such as weakness or loss of interjoint coordination resulting in deficits ranging from paralysis, abnormal movement patterns or pathological synergies (e.g. coupling of shoulder abduction and elbow flexion) to a certain degree of inter-joint coordination (Laghorne et al., 2011; Santello and Lang, 2015). In consequence, stroke subjects with arm impairments can show a reduced adaptability to task demands (Santello and Lang, 2015), inefficient movement trajectories (Osu et al., 2011), higher energy and force-consumption (Suresh et al., 2008) (when for example trying to perform goal-directed reaching movements), and the increased risk of frustration in case of unsuccessful movement attempts (Kitago et al., 2013; Raghavan, 2015). Hence, these impairments lead to long-term disabilities contributing in making stroke one of the main causes of disability worldwide, with a tremendous socio-economic impact (Feigin, 2014). Being able to evaluate stroke-specific upper limb movement patterns with sufficient detail is critical to properly monitor upper limb impairments after stroke, the recovery thereof and determining the effectiveness of different treatment approaches (Kwakkel et al., 2017). There exist multiple different standard clinical upper limb assessments in stroke research, among which the Fugl-Meyer Assessment of the Upper Extremity (FMA-UE) (Fugl-Meyer et al., 1975) is widely used to describe voluntary movement control (Santisteban, 2016). This is assessed in a hierarchical structure; from within to out-of synergistic movements and from proximal to distal upper extremity segments. Despite the satisfactory measurement properties (Gladstone et al., 2002; See et al., 2013), most of these clinical assessments exhibit floor and ceiling effects (Thrane et al., 2019) and heavily rely on subjective observer scoring on broad ordinal scales (such as: $0=$ not, $1=$ partially, $2=$ fully possible).

Novel technologies for kinematic signal detection and processing provide the opportunity for an objective and accurate motion analysis, which allow to overcome the limitations of standard clinical assessments in stroke research and rehabilitation (Kwakkel et al., 2017). Among the different approaches, it is worth mentioning (i) device-based assessments (Lambercy et al., 2013; Basteris et al., 2018), and (ii) less interfering wearable systems for bio-signal and motion capture processing (Camardella et al., 2018; Lorussi et al., 2016). However, although promising, the 
state-of-the-art solutions need to face with important issues, such as operator and patient safety and costs of the procedure (i). Bio-signal measurements, especially kinematic recordings (ii), can offer a reliable way for motion evaluation during a set of natural movements. In particular, the wearable sensor-based approach has shown to be applicable to a varietyof movement tasks and expendable to daily-living tasks (Held et al., 2018), which increases the relevance of the assessment compared to the more abstract and stringent nature of movements performed during standard clinical assessments. Though some characteristics such as longer movement times and higher trunk displacement were described in stroke subjects (Alt Murphy and Häger, 2015), an exhaustive mathematical characterization on the level of loss of inter-joint coordination or pathological synergies after stroke still lacks in literature (Santello and Lang, 2015). The most common way to quantify synergies is through Principal Component Analysis (PCA), a statistical method that allows the identification of dominant variation patterns in the data (Heidari et al., 2016; Averta et al., 2018). Using this approach in a single-case study, fewer combinations of joint motions were identified in one stroke subject (Reisman and Scholz, 2003) as well as stronger synergistic coupling between shoulder, elbow and wrist motions in contrast to a healthy subject (Heidari et al., 2016). In van Kordelaar et al. (2012), the authors applied a PCA on a larger dataset of stroke subjects $(n=46)$ to investigate the components of linear relations between the upper limb joints and the trunk at the end of reaching movements. However, a common drawback of applying PCA is the underlying hypothesis of temporal uncorrelation of upper limb poses in time (Averta et al., 2017). Consequently, the dynamic aspects of upper limb motion including the temporal evolution of upper limb joint trajectories are neglected. To overcome this issue, in Averta et al. (2017) the authors proposed functional Principal Component Analysis (fPCA) as a technique to investigate the dominant modes of time-dependent variation upper limb movements on a comprehensive set of upper limb dailyliving activities in healthy subjects. The main advantage of this analysis is that, while classical PCA-based analysis consider single kinematic postures, with fPCA all the temporal evolution of the movement is considered (thus intrinsically including the dynamic aspects). Results showed that a reduced number of functional Principal Components (fPCs) can be used to describe and accurately reconstruct the complexity of upper limb activities in healthy subjects, at joint level. The authors also pointed to the possibilities of an automatic recognition of physiological and pathological movements in stroke research and rehabilitation by analyzing fPCs variations between the affected and non-affected upper limb (Averta et al., 2017).

In the present study we investigate whether the outcomes discussed in Averta et al. (2017) can be effectively exploited to characterize upper limb motor impairment in chronic stroke 
subjects. For that purpose, four chronic stroke subjects with moderate arm and hand impairments performed the same 30 tasks of daily living activities (Averta et al., 2017) with both arms (affected and nonaffected) and a functional PCA analysis was applied. Based on this data set, we evaluated whether the set of fPCs and the associated approximation error in reconstructing joint trajectories are appropriate outcome measures to differentiate i) between the affected and the non-affected arm across stroke subjects and ii) between different levels of upper limb impairments of the single subjects

\subsection{Experimental protocol and setup}

\subsubsection{Set of daily living tasks}

In Averta et al. (2017), the authors discussed a large set of tasks (i.e., 30 different actions), which were selected to excite the whole upper limb work-space (Lenarcic and Umek, 1994; Abdel-Malek et al., 2004; Perry et al., 2007) and to span all the major hand configurations, e.g. referring to the most common hand grasping taxonomies (Feix et al., 2016; Cutkosky, 1989). Leveraging on this, we employed here the same protocol, with the twofold goal to: i) provide a comparison between different groups of populations, including chronic stroke subjects in this case, and ii) contribute to building up a large experimental data set of upper limb motion (acquired using different acquisition modalities), to enable comparison and benchmarking in future works. Under this regard, the possibility to publicly share collected data is envisioned and already under evaluation. The task-set is divided in three sub-groups

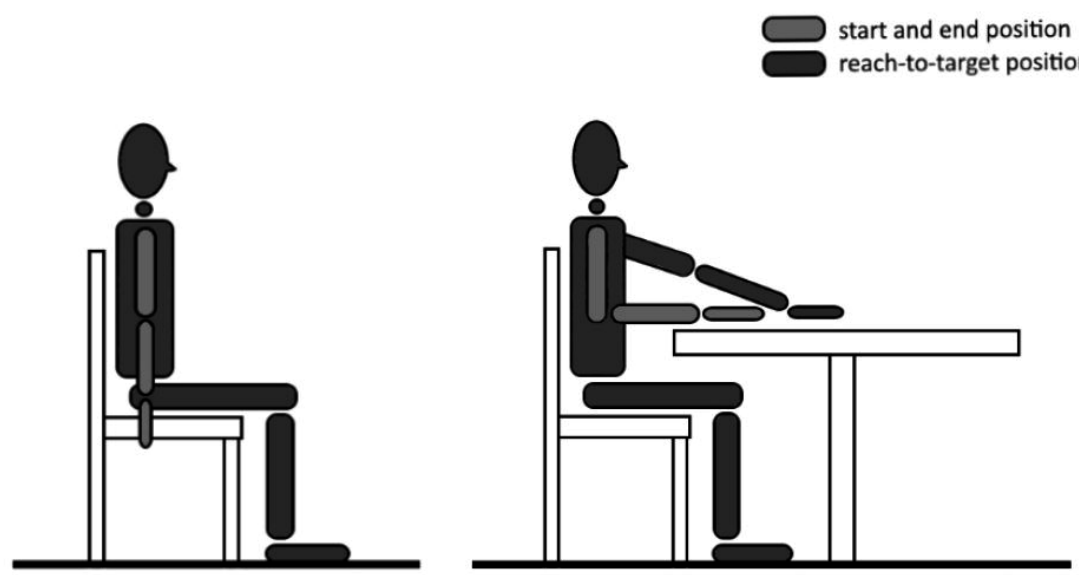

Figure 4.1. Overview of the experimental setup for the intransitive task (left) and transitive and toolmediated task (right). 
of ten actions each: intransitive, transitive and tool-mediated tasks. Actions included in the first group are gestures with no contact with the external environment (i.e., requiring movement of the proximal part of the upper limb), while the second group contains actions that involve interaction with an object. The third group implies tasks in which one external object is used to act on the environment. A detailed description of the taskset can be found in Averta et al. (2017) and Catrambone et al. (2019). In contrast to standard clinical scales that mostly consist of abstract movement executions and postures, the execution of a set of daily living tasks increases the meaningfulness of arm and hand movements, and thereby the ecological validity of measurements.

\subsubsection{Experimental setup for data acquisition}

The data were recorded with a full-body worn IMU-based system sensor suit (Xsens technologies B.V., Enschede, The Netherlands). The system consists of 17 inertial measurement units (IMUs) placed symmetrically on predefined body positions and fixed with Velcro straps and a size-fitting T-Shirt. The IMUs provide 3D angular velocity using rate gyroscopes, $3 \mathrm{D}$ acceleration using accelerometers, 3D earth magnetic field using magnetometers, as well as atmospheric pressure using the barometer in an operating frequency $2405-1475 \mathrm{MHz}$ [29]. A calibration procedure was required to evaluate sensors drifts and internal parameters. This was achieved using information related to subjects' body dimensions and through data fusion. The calibrated model was then used to reconstruct the whole body kinematic description. In particular, 23 links (or segments) connected through 22 spherical joints are used to model the human body. Several relevant motion-related quantities are then calculated and provided as output with a recording frequency of $60 \mathrm{~Hz}$, such as segments and joint position, velocities, and accelerations (see Figure 4.2). More details can be found in the MVN User Manual (2017). Once the system is calibrated, the experimental procedure established the repetition of each task of the protocol three times. All measurements were performed in upright sitting position on a chair. Subjects were instructed to perform each movement task at comfortable speed, first using the non-affected and then the affected upper limb. Task actions including grasping and manipulating objects (e.g. transitive and tool-mediated task group, see Averta et al. (2017) and Catrambone et al. (2019) for more details) were performed in front of a height-adjustable table (at forearm height in 90 elbow flexion and neutral shoulder position) with the targets placed at about 90 percent of the arm length (shoulder joint-axis until line of proximal interphalangeal joints). 


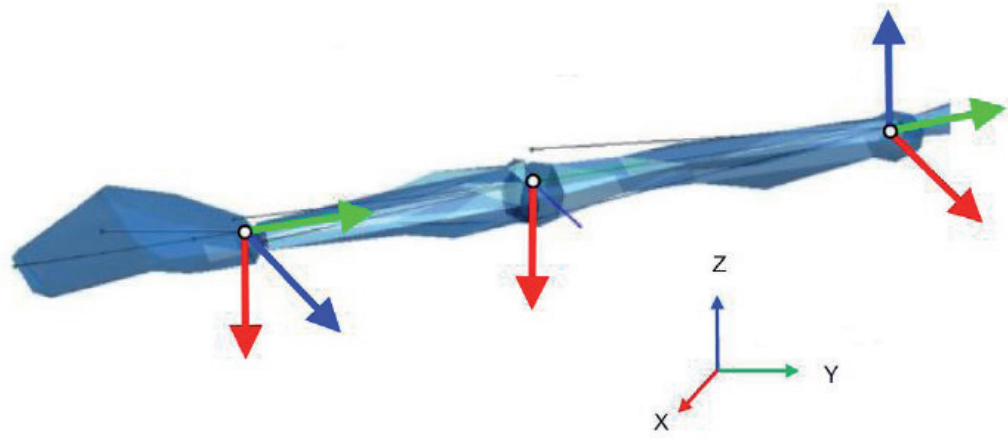

Figure 4.2. Kinematic model used in this work.

Three rigid links are connected by seven joints. Picture adapted from Averta et al. (2017).

\subsubsection{Study information}

In this work, we use data recorded from a subset of four chronic stroke subjects, which are part of the observational study "Assessing pathological synergies of upper limb function and the relationship to visuospatial function after stroke". All subjects gave written consent in accordance with the current version of the Declaration of Helsinki and the Swiss regulatory authority requirements. The protocol was approved by the Cantonal Ethics Committee Northwest and Central Switzerland (BASEC-ID: 2016-02075) and registered on ClinicalTrials. gov (Identifier: NCT03135093). Subjectspecific characteristics are reported in Table 4.1.

\subsection{Data analysis}

\subsubsection{Modeling and pre-processing}

We decided to use the XZY Euler parametrization to represent the data. In this way, we obtained nine angles in total to describe upper limb kinematics, three for the shoulder, three for the elbow and three for the wrist. Additional details on angles identification are provided in the MVN User Manual (2017). Without any loss of generality and to allow future comparisons, we chose to represent data with a 7 DoF model coherent with Averta et al. (2017). For these reasons, we considered only one DoF out of the three provided for the elbow (i.e. flexion-extension), three DoFs for the shoulder, and three DoFs for the wrist (of which one is related to forearm pronation-supination) (see Figure 4.2). Recorded data were manually segmented, meaning that for every data stream, we selected initial and final frames of each task repetition. Then, we linearly warped in time all the segments through 


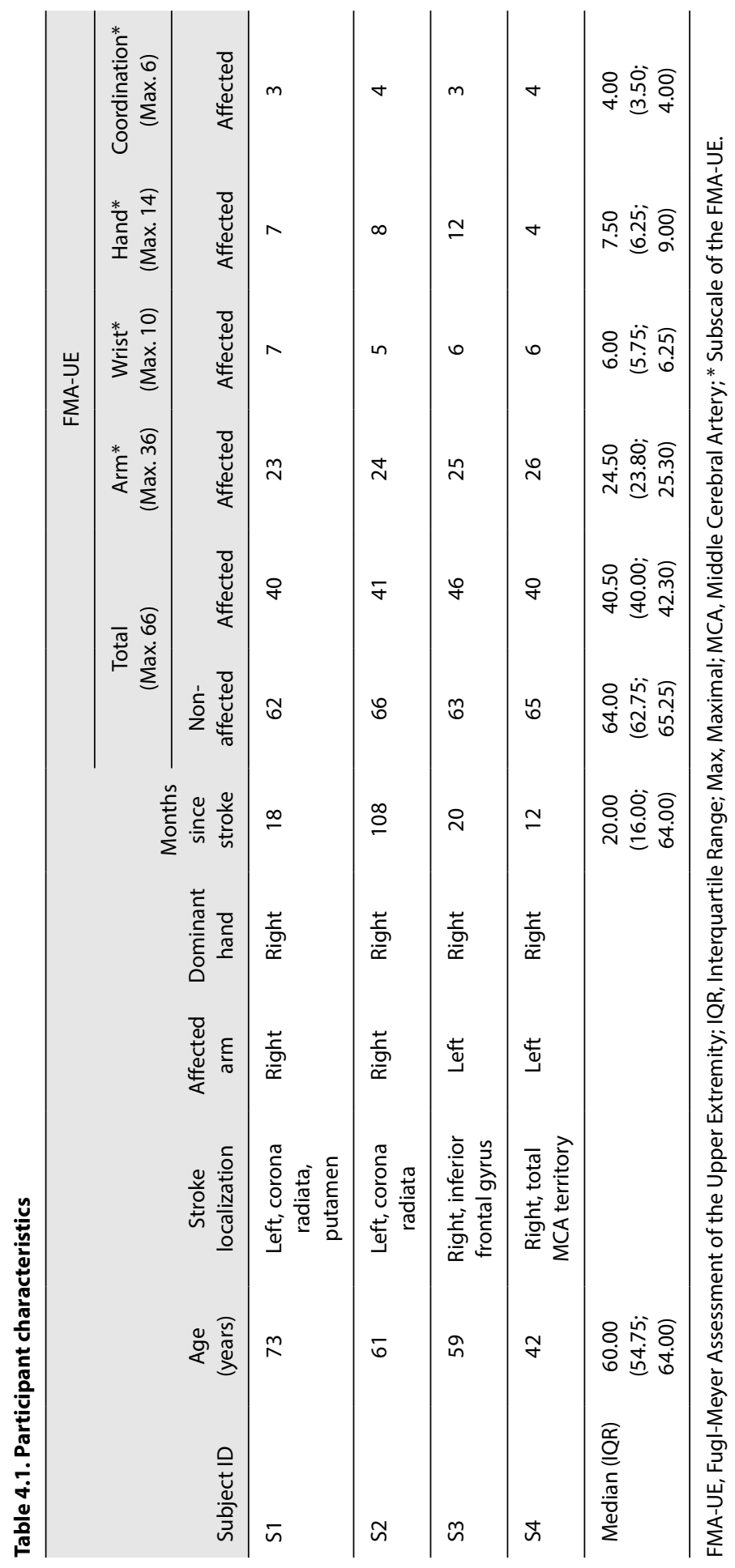


a re-sampling procedure with respect to a fixed number of time frames $(\mathrm{T}=300)$. This was made to enable time-comparison of different movements and allow a proper implementation of functional analysis (see Averta et al., 2017).

\subsubsection{Evaluation-index of motion complexity}

After the pre-processing phase, for each task, we got the temporal evolution of each joint normalized in time. To quantify the complexity of these movements under a functional point of view, a possible strategy is to evaluate how many basis functions are required to reconstruct the specific joint trajectories. Functional Principal Component Analysis (fPCA) represents a classic approach to identify the main modulating functions of one data set, and to order these in a descending order related to the explained variance. IPCA is a functional extension of Principal Component Analysis (PCA), typically used in several research fields to analyze multidimensional time series (Ramsay, 2006). More recently, this technique has been profitably applied in Averta et al. (2017) to identify the main functions that modulate human movements.

In the following, we will briefly describe the main idea behind PPCA and how it has been applied for motion description. Let us assume, without any loss of generality, a 7 DoF kinematic model to represent upper limb joint trajectories $q(t): R \rightarrow R^{7}$ where $t \in|0,1|$ is the normalized time.

In these terms, generic upper limb motion $q(t)$ can be decomposed in terms of the weighted sum of base elements $S_{i}(t)$, or functional synergies

$$
q(t) \cong \bar{q}(t)+S_{0}(t)+\sum_{i=1}^{S_{\max }} \alpha_{i} o S_{i}(t)
$$

where $\alpha_{i} \in R^{k}$ is a vector of weights, $S_{i}(t) \in R^{n}$ - in our case n equals to 7 - is the $i^{\text {th }}$ basis element or synergy and $S_{\max }$ is the number of basis elements. The operator $o$ is the elementwise product (Hadamard product), and $\bar{q} \in R^{7} 2 \mathrm{R} 7$ is the average posture of $q$

$$
\bar{q}=\int_{0}^{1} q(\tau) d \tau,
$$

while $S_{0}: R \rightarrow R^{7}$ is the average trajectory, also called zero-order synergy. The output of fPCA is a basis of functions $\left\{S_{1}, \ldots, S_{S_{\max }}\right\}$ that maximizes the explained variance of the movements in the collected dataset. Given a dataset with $N$ elements, the first $\operatorname{fPC} S_{1}(t)$ is the function that solves the following problem 
$\max$

$S_{1}$

$$
\sum_{j=1}^{N}\left(\int S_{1}(t) q_{j}(t) d t\right)^{2}
$$

subject to

$$
\left\|S_{1}(t)\right\| \frac{2}{2}=\int_{0}^{1} S_{1}^{2}(t) d t=1 .
$$

Subsequent fPCs $S_{i}(t)$ are the functions that solve the following:

$$
\begin{array}{lc}
\max _{1} & \sum_{j=1}^{N}\left(\int S_{1}(t) q_{j}(t) d t\right)^{2} \\
\text { subject to } & \left\|S_{1}(t)\right\| \frac{2}{2}=1 \\
& \int_{0}^{1} S_{i}(t) S_{p}(t) d t=0, \forall_{p} \in\{1, \ldots, i-1\} .
\end{array}
$$

A detailed implementation of this method - which bypasses the solution of the minimization problem - is discussed in Averta et al. (2017). In this paper we used $s_{\max }=15$.

It has been proved that the higher is the number of fPCs used to reconstruct a signal, the lower is the error obtained for reconstructing the real data. This observation implies that also the complexity of a sample could be quantified in terms of the number of functional components needed to provide reliable

reconstruction. Indeed, given an arbitrary reconstruction error threshold, the higher is the variability of a time series, the higher is the number of fPCs required to approximate the signal with an error lower than the threshold.

In this paper, we propose to exploit these characteristics to quantify the differences, in terms of functional complexity, between two different physical conditions, i.e. non-affected vs. affected upper limb movements. The main hypothesis is that an affected motion, due to the loss of inter-joint coordination or - in other words - increased joint coupling, leads to more simple and less variable joint contributions than the normal condition (Santello and Lang, 2015), when analyzed on the same task-set of upper limb activities. In our analysis, this should be reflected in the fact that, given a specific number of fPCs used to reconstruct a signal, the reconstruction error will be lower in case of pathological movements. Given $d_{i}$ as the $i^{\text {th }}$ element of the dataset, and given the first k synergies, the approximation error is evaluated as

$$
e_{k}(i)=\min _{\alpha_{j, i}} \quad\left[r m s\left(q_{i}(t)-\hat{q}_{i}^{k}\left(t, \alpha_{j, i}\right)\right)\right]
$$


where $\hat{q}_{i}^{k}\left(t, \alpha_{j, i}\right)$ is the approximation of $q_{i}(t)$ using the first $k$ synergies, calculated as

$$
\hat{q}_{i}^{k}(t)=\bar{q}(t)+S_{0}(t)+\sum_{j=1}^{k} \alpha_{j, i} \text { o } S_{j}(t),
$$

and $\alpha_{j, i}$ are the optimal weights associated to the element $d_{i}$. Representative error values for each $k$ are then calculate as the average $\bar{e}_{k}=\operatorname{mean}\left(\left[e_{k}(1), \ldots, e_{k}(N)\right]\right)$ and collected in a vector $E=\left[\bar{e}_{1}, \ldots, \bar{e}_{S_{\max }}\right]$.

A schematics of this idea is depicted in Figure 4.3, where the red plot refers to affected motion, while the green plot refers to the non-affected motion. The blue area within the two profiles can be regarded as an index of dissimilarity between the two conditions. The dissimilarity index $\left(I_{D}\right)$ can be easily calculated as:

$$
I_{D}=\sum_{i=1}^{s_{\max }} E_{H}(i)-\sum_{j=1}^{s_{\max }} E_{S}(i)=\sum_{i=1}^{s_{\max }}\left(E_{H}(i)-E_{S}(i)\right)
$$

where $E_{H}$ is the vector of reconstruction error for the healthy case, $E_{S}$ is the vector of reconstruction error for the stroke case.

The procedure to obtain the plots theorized in Figure 4.3 and to calculate $I_{D}$ is reported in Algorithm 4.1. Please note that under a theoretical point of view, this methodology could be generalized to the analysis of different types of motion datasets with multiple subjects, and applied to intra-subject analysis.

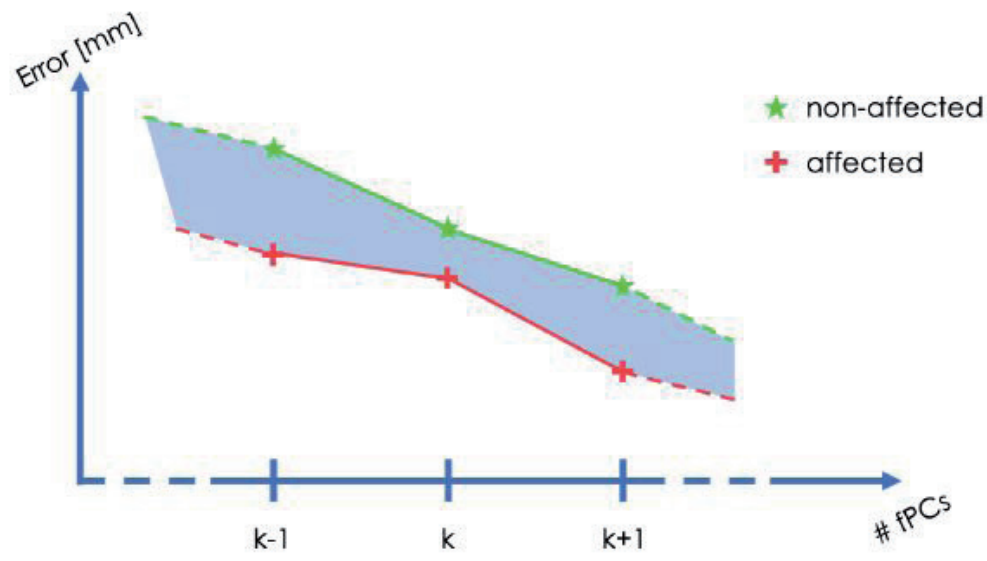

Figure 4.3. Typical profiles of reconstruction error w.r.t. the nuber of fPCs enrolled.

Red and green lines are the expected shapes in case of affected and healthy motion respectively. The area between the two curves can be intended as an index of dissimilarity between the two conditions. 


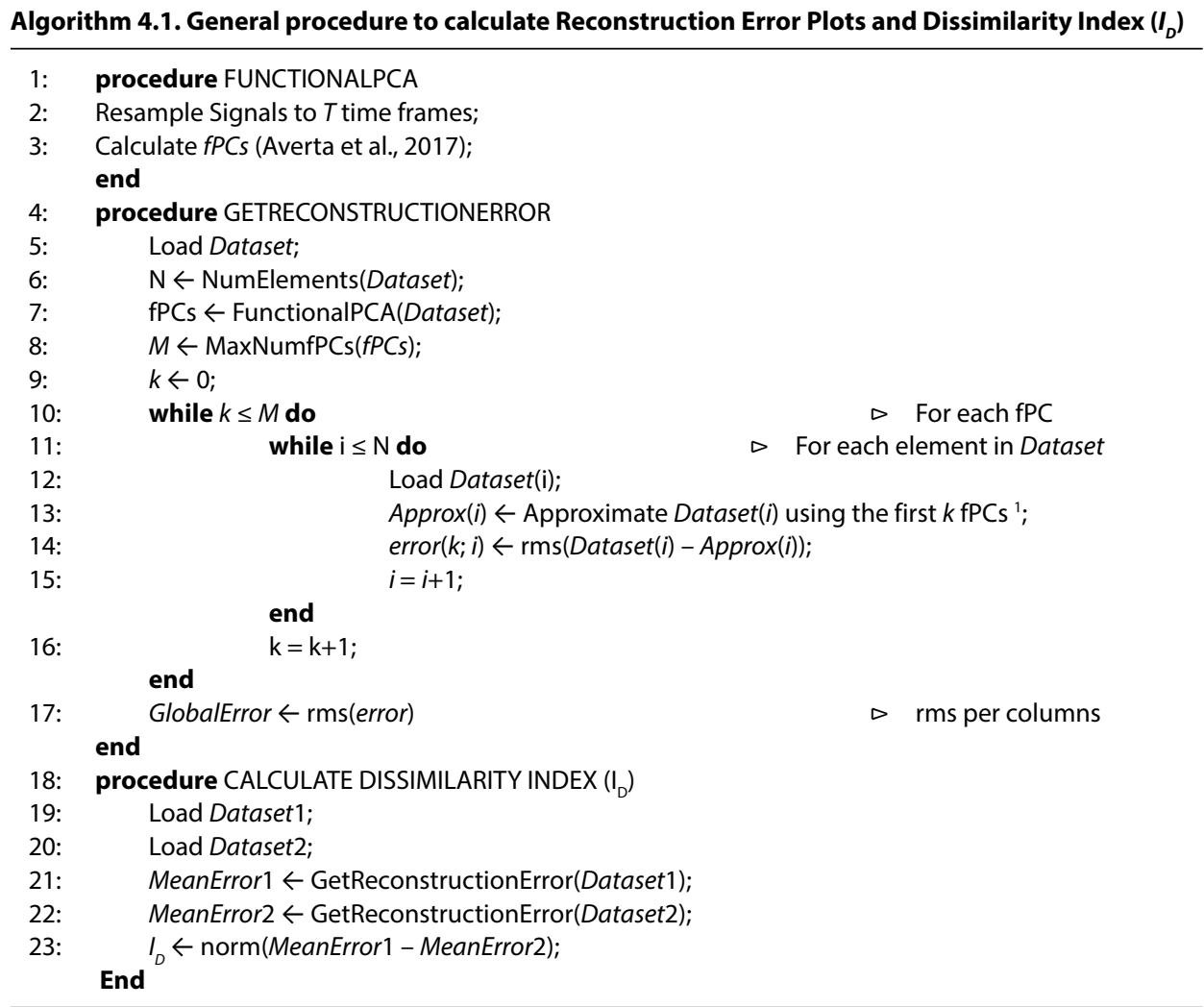

\subsection{Results and discussion}

To verify whether our main hypothesis holds true, we calculated the dissimilarity index $\left(\mathrm{I}_{\mathrm{D}}\right)$ defined in the previous section between the whole data-set of non-affected arm motions and the whole data-set of affected arm motions. Results are depicted in Figure 4.4 for all the stroke subjects. It is possible to observe that the reconstruction error is lower for the affected upper limb, as per research hypothesis discussed in the previous section. The blue area can be used as a gross difference index between the affected and nonaffected conditions for all stroke subjects in the whole data sample (ID=14.703). This result supports our assumption that analysis of movement variability can be used to effectively distinguish between nonaffected and affected conditions due to stroke.

To further characterize the proposed ID as index of the impairment level, we performed the same analyses on a singlesubject-level. In order to evaluate whether our approach is able to differentiate between subjects and their individual impairment severity, we repeated 


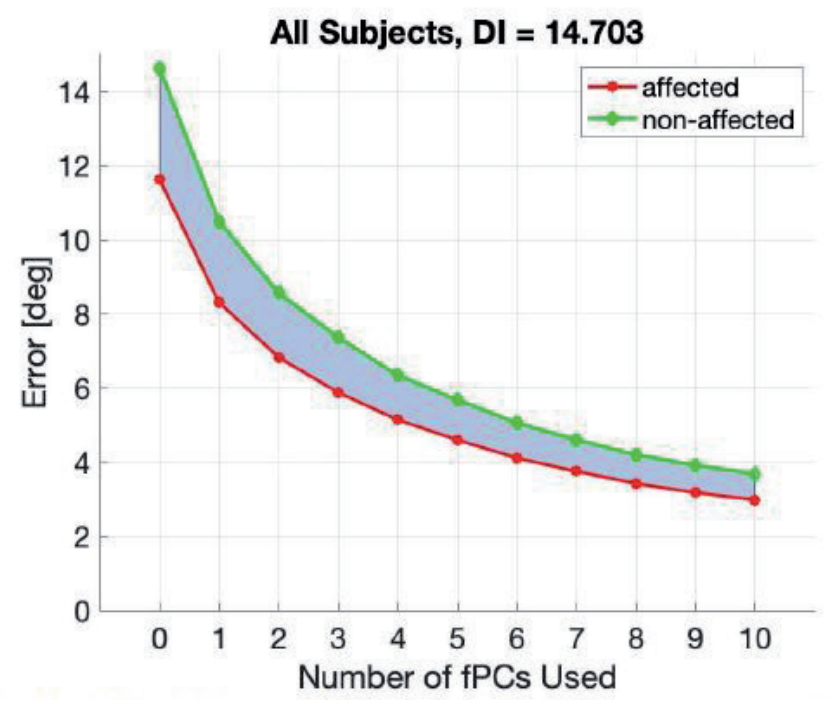

Figure 4.4. Root Mean Square of reconstruction error vs. number of fPCs used. All subjects are considered for this analysis. $\mathrm{Dl}=14.703$.

our procedure to each single-subject's data-set. Results, reported in Figure 4.5, illustrate differences in terms of movement variability between the affected and non-affected side of each subject. While our main hypothesis of lower reconstruction errors in the affected arm holds true for S1, S2 and S3 (ID ranging from 12.12 to 27.31), higher reconstruction errors are visible in the affected arm of $\mathrm{S} 4$ when compared to the non-affected side leading to a negative ID equal to -6.91 . We believe that, this higher variability in the affected arm is related to extra-movements, which $\mathrm{S} 4$ performed to compensate for the diminished grasp function (FMA-UE hand score: 4 out of 14) when performing the transitive and tool-mediated task actions. To verify this hypothesis, we performed the same approach for the subset of intransitive task actions, where finger and hand functions are not crucial for the task accomplishment, for all the considered subjects. Almost equal variability profiles between the affected and non-affected arm and a ID of 1.27 were found in S4, supporting the idea of approximately normal movement behavior as long as no hand or finger function is needed, whereas the movement variability seems to be comparably diminished in S1 (ID of 11.65), S2 (ID of 24.33) and S3 (ID of 12.60). Illustrations of these analyses are omitted for sake of space. 

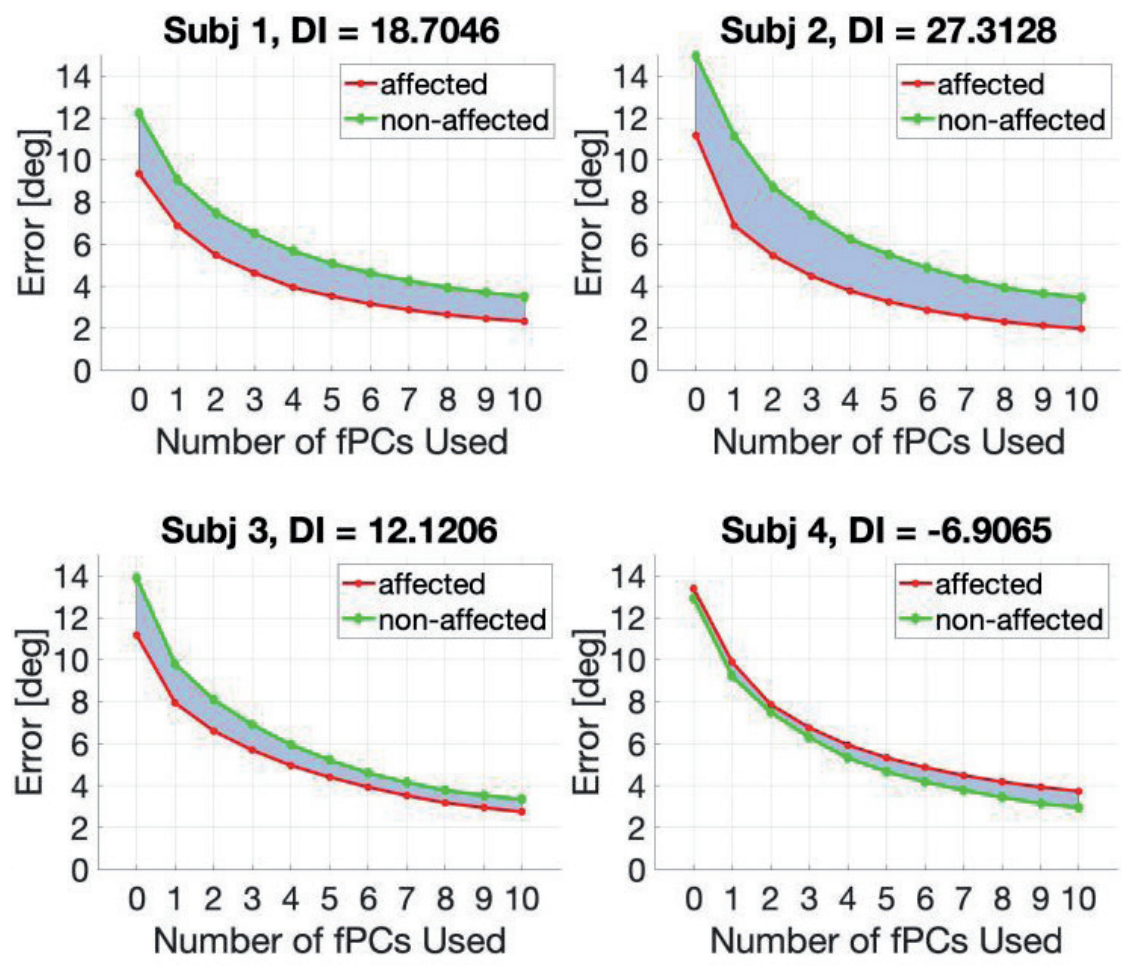

Figure 4.5. Root Mean Square of reconstruction error vs. number of fPCs used.

Each sub-plot reports the analysis subject-specific. From top-left to bottom-right results for subjects from 1 to 4 are considered. Correspondent DI is reported in figure headings.

\subsection{Conclusion}

In this proof-of-principle work, we demonstrated how stroke subjects' level of impairment may be assessed through the quantification of upper limb movement variability, in terms of the resulting root mean square of reconstruction error using a fPCs based description. The ID calculated upon the whole dataset revealed differences in terms of motion variability between the two upper limb conditions. In this manner, novel measurement outcomes are provided to describe strokerelated upper limb movement impairments, such as the loss of inter-joint coordination, commonly associated with reduced variability of motion elements, when performing a comprehensive set of daily living activities. This opens fascinating perspectives toward the usage of this methodology as a tool for assessing motor impairment after a stroke, herein defined as reduced movement variability, in a quantitative, sensitive and operator-independent fashion. Although only preliminary, results seem confirming our hypothesis of generally lower profiles of reconstruction errors in the affected compared to 
the non-affected upper limb (as shown in Figure 4.4 by comparing both conditions for the whole set of four subjects as shown in Figure 4.5). The single-subject analysis revealed a ID ranging from 27.31 in S2 to -6.91 in S4, that can partially be explained by clinical assessment outcomes as shown in Table 4.1. Studies on a larger data set are needed to confirm these preliminary findings, also including other motor impairments, such as ataxia, which would likely influence the results and interpretation of the presented outcomes. These findings suggest the usefulness of fPCA to study the motion variability and provide implications for sensitive outcomes of post-stroke xupper limb impairment, which may be effectively used as suitable biomarkers to discriminate between pathological and physiological movement behaviour in stroke research and rehabilitation [12]. In [27] the authors similarly found correct predictions of the presence or absence of basic limb synergies (defined by FMA-UE 34 and FMA-UE $<34$ ) for 38 of 46 patients $(82: 6 \%)$ using PCA on endpoint reaching kinematics. Here, we extend the PCA to the whole time set of motion data using shape analysis described in functions, which enables the investigation of dynamic aspects of movement behavior, including the spatio-temporal evolution of joint trajectories to precisely describe pathological joint coupling or pathological synergies due to stroke. However, we acknowledge that, in contrast to the classical PCA performed at one point in time, direct coupling between joints are not explicitly considered with our approach. As another limitation, we acknowledge that limiting the application of the approach to the upper limb except for the hand and fingers could lead to erroneous assessment and interpretation of the results. For this reason, model extensions to hand and finger motions will be considered to additionally include relevant kinetic information for grasping activities. Finally, our future works will also take into account possible deficits in the non-affected arm. Considering previous works on physiological movement datasets (Averta et al., 2017), here, we could broaden the analysis to pathological movement data from mildly to moderately affected stroke subjects, when performing a set of daily living tasks. In a next step, differences between specific single taskitems will be investigated using the same methodology. Finally, this methodology could be further exploited for an automatic recognition of physiological and pathological movements through machine learning and in terms of online evaluations of improvements related to rehabilitation procedures. 


\subsection{References}

Abdel-Malek K, Yang J, Brand R, Tanbour E. Towards understanding the workspace of human limbs. Ergonomics. 2004;47:1386-405.

Alt Murphy M, Häger CL. Kinematic analysis of the upper extremity after stroke-how far have we reached and what have we grasped? Phys Ther Rev. 2015;20:137-55.

Averta G, Angelini F, Bonilla M, Bianchi M, Bicchi A. Incrementality and hierarchies in the enrollment of multiple synergies for grasp planning. IEEE Robot Autom Lett. 2018;3:2686-93.

Averta G, Della Santina C, Battaglia E, Felici F, Bianchi M, Bicchi A. Unvealing the principal modes of human upper limb movements through functional analysis. Front Robot AI. 2017;4:37.

Basteris A, Contu S, Plunkett TK, Kuah CW, Konczak IJ, Chua KS, Masia L. Robot-aided bimanual assessment of wrist proprioception in people with acute stroke. 2018 7th IEEE International Conference on Biomedical Robotics and Biomechatronics (Biorob). IEEE; 2018 (pp. 473-8). doi: 10.1109/BIOROB.2018.8487987

Camardella C, Murciego LP, Tang S, Bertolucci F, Chisari C, Barsotti M, Frisoli A. Simple tool for functional and physiological stroke patients assessment. International Conference on NeuroRehabilitation. Springer; 2018 (pp. 779-82).

Catrambone V, Greco A, Averta G, Bianchi M, Valenza G, Scilingo EP. Predicting object-mediated gestures from brain activity: an EEG study on gender differences. IEEE Trans Neural Systems. 2019:1.

Cutkosky MR. On grasp choice, grasp models, and the design of hands for manufacturing tasks. IEEE Trans Robot. 1989;5:269-79.

Della Santina C, Bianchi M, Averta G, et al. Postural hand synergies during environmental constraint exploitation. Front Neurorobot. 2017;11:41.

Feigin VL, Forouzanfar MH, Krishnamurthi R, et al. Global and regional burden of stroke during 1990-2010: findings from the global burden of disease study 2010. Lancet. 2014;383:245-55.

Feix T, Romero J, Schmiedmayer HB, Dollar AM, Kragic D. The grasp taxonomy of human grasp types. IEEE Trans Hum Mach Syst. 2016;46:66-77.

Fugl-Meyer AR, Jääaskö L, Leyman I, Olsson S, Steglind S. The post-stroke hemiplegic patient. 1. a method for evaluation of physical performance. Scand J Rehabil Med. 1975;7:13-31.

Gladstone DJ, Danells CJ, Black SE. The Fugl-Meyer assessment of motor recovery after stroke: a critical review of its measurement properties. Neurorehabil Neural Repair. 2002;16:232-40.

Heidari O, Roylance JO, Perez-Gracia A, Kendall E. Quantification of upper-body synergies: A case comparison for stroke and non-stroke victims. ASME 2016 International Design Engineering Technical Conferences and Computers and Information in Engineering Conference. American Society of Mechanical Engineers; 2016 (pp. V05AT07A032-V05AT07A032).

Held JP, Klaassen B, Eenhoorn A, van Beijnum BJF, Buurke JH, Veltink PH, Luft AR. Inertial sensor measurements of upperlimb kinematics in stroke patients in clinic and home environment. Front Bioeng Biotechnol. 2018;6:27.

Kitago T, Ryan SL, Mazzoni P, Krakauer JW, Haith AM. Unlearning versus savings in visuomotor adaptation: comparing effects of washout, passage of time, and removal of errors on motor memory. Front Hum Neurosci. 2013;7:307. 
Kwakkel G, Lannin NA, Borschmann K, et al. Standardized measurement of sensorimotor recovery in stroke trials: consensus-based core recommendations from the stroke recovery and rehabilitation roundtable. Neurorehabil Neural Repair. 2017;31:784-92.

Lambercy O, Fluet MC, Lamers I, Kerkhofs L, Feys P, Gassert R. Assessment of upper limb motor function in patients with multiple sclerosis using the virtual peg insertion test: a pilot study. IEEE Int Conf Rehabil Robot. 2013;2013:6650494.

Langhorne P, Bernhardt J, Kwakkel G. Stroke rehabilitation. Lancet. 2011;377:1693-702. Latash NL. Synergy. Oxford University Press; 2008.

Lenarcic J, Umek A. Simple model of human arm reachable workspace. IEEE Trans Syst. 1994;24:123946.

Lorussi F, Carbonaro N, De Rossi D, Paradiso R, Veltink P, Tognetti A. Wearable textile platform for assessing stroke patient treatment in daily life conditions. Front Bioeng Biotechnol. 2016;4:28.

MVN User Manual. User Guide MVN, MVN BIOMECH MVN Link, MVN Awinda. Rev. March. Xsens Technologies B.V.; 2017.

Osu R, Ota K, Fujiwara T, Otaka Y, Kawato M, Liu M. Quantifying the quality of hand movement in stroke patients through threedimensional curvature. J Neuroeng Rehabil. 2011;8:62.

Perry JC, Rosen J, Burns S. Upper-limb powered exoskeleton design. IEEE ASME Trans Mechatron. 2007;12:408.

Raghavan P. Upper limb motor impairment after stroke. Phys Med Rehabil Clin M Am. 2015;26:599610.

Ramsay JO. Functional data analysis. Wiley Online Library; 2006.

Reisman DS, Scholz JP. Aspects of joint coordination are preserved during pointing in persons with post-stroke hemiparesis. Brain. 2003;126:2510-27.

Santello M. Synergistic control of hand muscles through common neural input. In The Human Hand as an Inspiration for Robot Hand Development. Springer; 2014 (pp. 23-48).

Santello M, Lang CE. Are movement disorders and sensorimotor injuries pathologic synergies? when normal multi-joint movement synergies become pathologic. Front Hum Neurosci. 2015;8:1050.

Santisteban L, Térémetz M, Bleton JP, Baron JC, Maier MA, Lindberg PG. Upper limb outcome measures used in stroke rehabilitation studies: a systematic literature review. PloS One. 2016;11:e0154792.

See J, Dodakian L, Chou C, Chan V, McKenzie A, Reinkensmeyer DJ, Cramer SC. A standardized approach to the fugl-meyer assessment and its implications for clinical trials. Neurorehabil Neural Repair. 2013;27:732-41.

Suresh NL, Zhou P, Rymer WZ. Abnormal EMG-force slope estimates in the first dorsal interosseous of hemiparetic stroke survivors. Ammu Int Conf IEEE Eng Med Biol Soc. 2008;2008:3562-5.

Thrane G, Sunnerhagen KS, Persson HC, Opheim A, Alt Murphy M. Kinematic upper extremity performance in people with near or fully recovered sensorimotor function after stroke. Physiother Theory Pract. 2019;35:822-32.

Tresch MC, Jarc A. The case for and against muscle synergies. Curr Opin Neurobiol. 2009;19:601-7. van Kordelaar J, van Wegen EE, Kwakkel G. Unraveling the interaction between pathological upper limb synergies and compensatory trunk movements during reach-to-grasp after stroke: a crosssectional study. Exp Brain Res. 2012;221:251-62. 



\title{
Kinematic core-set of upper limb movements after stroke and their relationship across various upper limb activities of life
}

\author{
A. Schwarz \\ M.M.C. Bhagubai \\ S.G. Nies \\ J.P.O. Held \\ P.H. Veltink \\ J.H. Buurke \\ A.R. Luft
}




\subsection{Abstract}

Background - Kinematic assessments provide quantifiable information on qualitative movement behavior after stroke. A comprehensive characterization of spatiotemporal kinematics of stroke subjects during upper limb activities of daily living is lacking. Differences of kinematic expressions were investigated with respect to the movement type and the impairment level for the entire task and sub phases representing building blocks or motion primitives. It was questioned whether motion primitives of reaching distally and proximally are comparable across tasks.

Method - Chronic stroke subjects with upper limb movement impairments and healthy subjects performed a set of 20 daily living activities including gesture and grasp movements. Kinematics of trunk displacement, shoulder flexion/extension, shoulder abduction/adduction, elbow flexion/extension, forearm pronation/supination, wrist flexion/extension, movement time, hand peak velocity, number of velocity peaks (NVP), and spectral arc length (SPARC) were extracted for the whole movement and the primitives of reaching distally and proximally. The effects on kinematics of the whole task were tested for the factors gesture versus grasp movements, and the impairment level. Similarities as metrics expressions and relations between the kinematics were investigated for the phases of reaching proximally and distally across subjects between tasks.

Results - Data of 26 stroke and 5 healthy subjects were included. All kinematic parameters were largely dependent on the factor gesture versus grasp movements with gestures resulting in larger shoulder joint motions and diminished distal arm motions beside higher peak velocity when compared to the grasp movements that were expressed by larger trunk, forearm, and wrist motions. Primitives of reaching distally were comparable across tasks in terms of trunk displacement, shoulder motions and peak velocity, while reaching proximally showed comparable expressions in trunk motions. For reaching distally comparable relations between metrics across tasks were found between shoulder flexion/extension and elbow flexion/extension, between peak velocity and elbow and shoulder flexion/ extension, between movement time and the NVP, and between NVP and SPARC. Reaching proximally revealed reproducible correlations across tasks between movement time and NVP.

Conclusion - Spatiotemporal differences between contact and non-contact movements, as well as between impairment levels were confirmed. The hypothesis of consistent movement building blocks or motion primitives was supported by similar kinematic expressions and associations in reaching distally and proximally. 


\subsection{Introduction}

The human upper limb consists of seven main degrees of freedom, excluding the hand, that allow for highly variable movements and interactions with the environment. After stroke, this movement complexity can be affected due to a disruption in the cerebral sensorimotor networks that lead to inefficient or abnormal movement activation (Raghavan, 2015). Sensitive assessments of the motor function and influences of deficits on daily life functionality are important to drive interventions to improve functional restoration.

Over the last decades, upper limb kinematic assessments have been increasingly used as a primary or secondary outcome measure besides standard clinical assessments in randomizedcontrolled trials concerning stroke rehabilitation (Bang et al., 2015; Bartolo et al., 2017; Conrad et al., 2015; Corti et al., 2012; di Lazarro et al., 2016; Durham et al., 2014; Ellis et al., 2016; Housman et al., 2009, Hsieh et al., 2016; Kim et al., 2013; Quattrocchi et al., 2017). Interventions such as constraint induced movement therapy (Massie et al., 2009; Wu et al., 2007; Wu et al., 2012), trunk restraint training (Lima et al., 2014), robotic-assisted Training (Colombo et al., 2017; Mazzoleni et al., 2013; Wu et al., 2012), virtual reality training (Piron et al., 2010; Subramanian et al., 2013), bilateral arm training (Lin et al., 2010; van Delden et al., 2015; Wu et al., 2013), Botulinum toxin (Bensmail et al., 2010) and mirror therapy (Wu et al., 2013) were tested for their effectiveness. This tendency demonstrates the usefulness of kinematic assessments to complement the standard clinical assessments and their limitations, such as the broad level of movement evaluation. It has been shown that upper limb kinematic measurements allow to track many different motion aspects objectively and continuously (van Meulen et al., 2017). Alongside with this emerging field, the variability and heterogeneity of kinematic assessment protocols and chosen outcomes increased, making it difficult to interpret findings across studies (Kwakkel et al., 2017). A systematic review provided an overview of upper limb kinematic assessments after stroke with respect to the assessment task, the investigated kinematic outcomes, and their clinimetric properties, suggesting assessment considerations and kinematic coreset metrics, such as task / movement time, path length ratio, no. of velocity peaks, shoulder flexion/extension angle, trunk displacement and peak velocity (Schwarz et al., 2019). It has been shown that upper limb kinematic measurements after stroke were frequently conducted based on device or experimental setup restricted movements, such as reach-to-point, tracking, or reach-to-grasp movements, in relatively fixed measurement surroundings such as camera-based motion laboratories or robot-based measurement systems. The fact that the movement or task content has a strong impact on motor planning and behavior, as for example whether pointing, or grasping of simulated or 
real objects are investigated (McCrea et al., 2002; Alt-Murphy et al., 2015) might be one of the biggest barriers in the overall comparison of upper movement kinematics. Even though more complex natural tasks, such as the drinking task have been established to assess upper limb function in close to real-life situations and thereby to improve the ecological validity of the assessment (Alt-Murphy et al., 2018), it is unknown how the assessment outcomes relate to other functional upper limb movement tasks. Furthermore, most kinematic analysis is based solely on the reaching phase of a movement. Consequentially, other limitations, such as when moving the hand to the mouth, or manipulating objects, can be overseen. A wider set of different upper limb assessment activities, including non-contact movements such as gestures, or contact movements such as grasping activities, would increase the representativeness and comprehensiveness of the kinematic characterization of upper limb movement quality in daily life.

Recently, effort has been put into the development of a taxonomy for upper limb motion that subdivides motions based on the complexity and duration into activities, functional movements, and functional primitives (Schambra et al., 2019). The functional movement primitives, such as reaching or transporting, were suggested to be seen as building blocks or even more granular elements of motion, that are consistent across movements (Giszter et al., 2015). Observing upper limb motions on the level of the functional movement primitives or movement subphases can enable across task comparisons of movement quality and overcome issues of anatomical and task-related complexity, and thereby help to uncover pathophysiological mal-adaptations in movement behavior after stroke. In the present study, a set of 20 activities of daily life was used reflecting the main requirements of movement control of the human upper limb degrees of freedom (DOF) in terms of workspace, grasp configuration, interaction with the environment and complexity (Averta et al., 2017). We aimed to characterize and differentiate ADL movements, such as gesture and grasping actions with respect to the severity of stroke-related upper limb impairment, by use of a kinematic coreset presenting the main upper limb spatiotemporal kinematic characteristics. The first question was whether kinematic characteristics are different between no-contact-based gesture movements and contact-based grasping and whether significant effects can be found related to subgroups of no, mild and moderate upper limb impairment. The second question was attributed to the comparability of movement subphases, such as reaching, across upper limb actions. It was questioned whether phases of reaching distally towards ipsilateral maximum arm length and reaching or transporting proximally towards the head are consistent in terms of spatiotemporal kinematic expressions across different actions. 


\subsection{Methods}

A prospective cross-sectional study on chronic stroke subjects was performed to explore the relationship between upper limb function and activities as measured by clinical assessments and by a wearable sensor-based motion capture system. The study protocol was approved by the Cantonal Ethics Committee Northwest and Central Switzerland (BASEC-ID: 2016-02075), prospectively registered in ClinicalTrials.gov (NCT03135093) and performed between July 2017 and October 2019 at the rehabilitation clinic cereneo (Vitznau, Switzerland).

\subsubsection{Study participants}

The study sample consisted of 26 subjects with a unilateral ischemic or hemorrhagic stroke in the chronic stage ( $>6$ months) with presence of partial upper limb motor impairment, allowing to lift the arm at least partially against gravity (>30 degrees of shoulder flexion), and to flex and extend the fingers for basic grasp performance. The subjects were excluded, if increased upper limb muscle tone with limitations in range of motion (modified Ashworth Scale $\geq 3$ ), severe sensory deficits were present in the upper limb (Erasmus modifications to the revised Nottingham Sensory Assessment of 0 in one of the test regions), or in case of upper limb impairments unrelated to the neurological disease, such as preexisting orthopedic problems. Five age-matched healthy subjects without limitations in upper limb movements were included for acquisition of kinematic reference data. Each participant had to be able to understand and follow basic commands to perform the study experiments and to give written informed consent before inclusion, according to the Declaration of Helsinki and the Swiss regulatory authorities.

\subsubsection{Study experiments}

All study experiments were performed by an experienced research therapist during a singleday measurement at the rehabilitation clinic cereneo (Vitznau, Switzerland). After donning and calibration of the inertial sensor system, the subjects were asked to perform a set of upper limb activities with the affected upper limb or non-dominant side in healthy participants that were each repeated three times. The experimental task selection was based on previous works (Averta et al., 2017; Schwarz and Averta, 2019), consisting of ten intransitive, gesture movements, and ten transitive, reach-to-grasp and manipulation movements, as illustrated in Figure 5.1 and described in detail in Supplementary Table S5.1. 

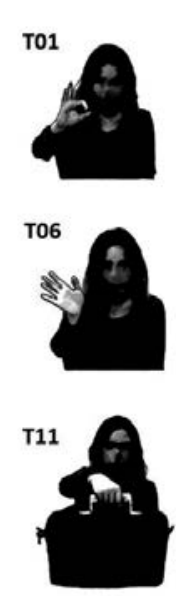

T16

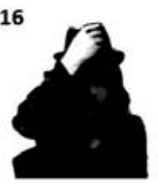

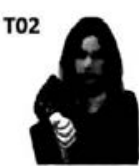
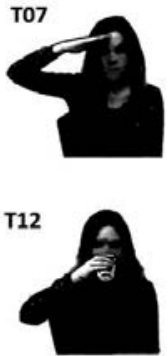

T17

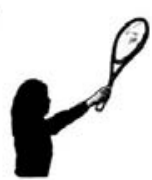

T03

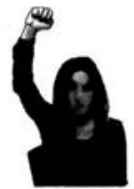

T08

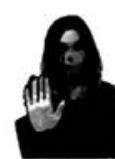

T13

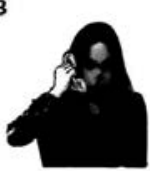

T18

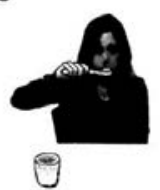

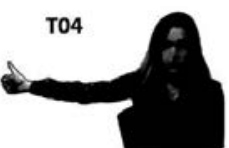

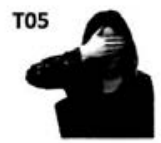

T09

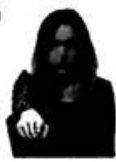

T10
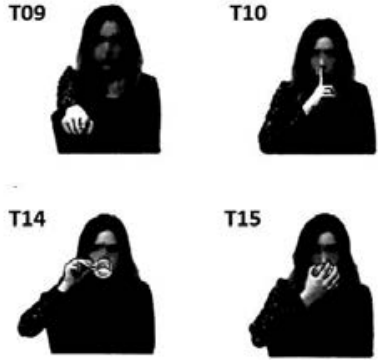

T15
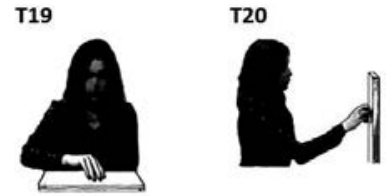

Figure 5.1. Experimental protocol of 20 activities of daily life (adapted from Averta et al., 2020).

This task set contains the main grasping types (Feix et al., 2016) and covers the main upper limb movement workspace, while enabling the differentiation between movement without contact, transitive actions with object contact, and tool-mediated actions, where the object is used to manipulate something. For testing similarities of motion primitives across tasks, four primitives, reaching distally to grasp, reaching distally to gesture, reaching proximally to transport, and reaching proximally to gesture, were defined, and tested in preselected tasks. Reaching distally to grasp and proximally to transport were detected in the tasks, T12, T13, T14, T15, T16, and T18. Reaching to gesture was tested in T02, T06, and T08, while reaching proximally to gesture was tested in T05, T07, and T10.

\subsubsection{Measurement system}

For primary outcome measures, kinematic data by use of a full-body, wearable motion capture system, Xsens MVN Awinda (Xsens Technologies, Enschede, the Netherlands), was recorded. The system offers real-time visualization, playback and editing of motion capture data by a set of 17 wireless sensors that were attached symmetrical onto predefined body-parts of the participant. For the purpose of this study, the sensors included for upper limb motion analysis were limited to the upper body, as illustrated in Figure 5.2. Each sensor contains sensors for 
recording $3 \mathrm{D}$ acceleration, $3 \mathrm{D}$ rotation and $3 \mathrm{D}$ earth-magnetic field direction to calculate subject-specific hand workspace and joint angles and positions (Roetenberg et al., 2007). The upper limb motion data captured with the Xsens MVN Awinda ranged from kinematics for maximum reach distance and movement range in vertical direction that have shown to correlate with the Fugl-Meyer Assessment of upper extremity (van Meulen et al., 2015) and offers objective measurements in everyday surroundings (Klaassen et al., 2017; Held et al., 2018).

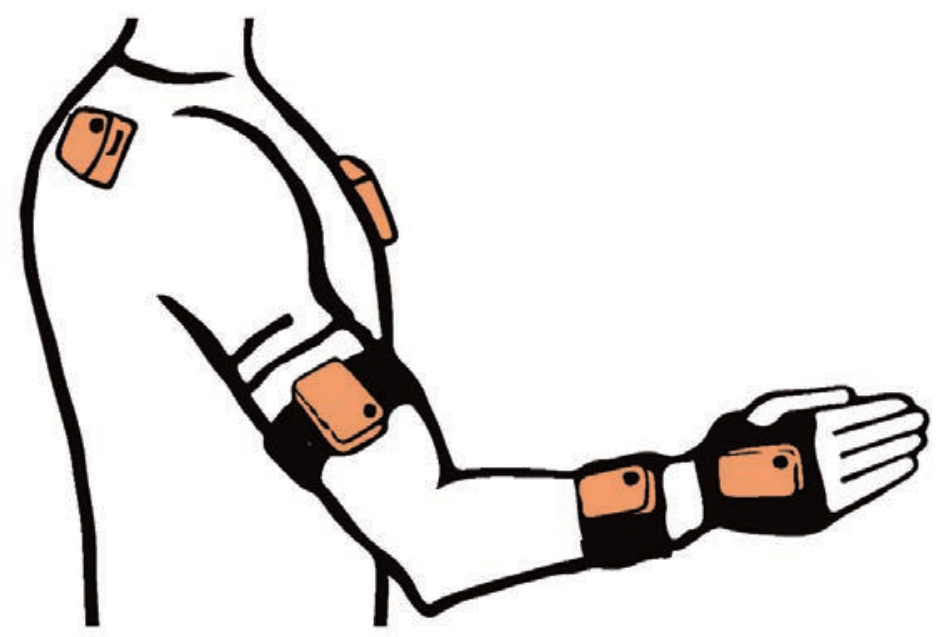

Figure 5.2. Upper limb sensor set-up.

\subsubsection{Kinematic core-set}

Spatiotemporal kinematic parameters of spatial posture (shoulder, elbow, wrist flexion/ extension, forearm pronation/supination, and trunk compensation), speed (peak velocity), smoothness (no of velocity peaks, spectral arc length), and movement time (MT) were evaluated to explore the metrics expressions across task groups, specific tasks and shared motion primitives of reach and transport in the subject-specific workspace. All kinematic measure processing steps were performed using Matlab (MATLAB version 2018b, The Mathwork, Natick, MA).

The spatial measures of range of motion in the shoulder, elbow and hand were defined by the scalar measure from minimum to maximum joint angle. As suggested in the MVN Xsens manual, the ZXY Euler rotation sequence is suggested to define the rotation axis of a joint, except of shoulder abduction/adduction angle that is recommended to be read out by the Euler rotation sequence XZY, that reduces issues of estimation errors due to gimbal 
lock (MVN Manual 2018). Shoulder flexion/extension is determined by rotation around the $\mathrm{z}$-axis of the Euler sequence ZXY along the sagittal plane. Shoulder abduction/adduction is defined by rotations around the y-axis of the Euler sequence XZY along the frontal plane. Elbow flexion/extension is defined by rotation around the $\mathrm{x}$-axis. Forearm pronation/ supination is defined around the y-axis and wrist flexion/extension by around the around the $\mathrm{x}$-axis. Trunk compensation is measured by changes in position and orientation of the sternum sensor (Subramanian et al., 2010). The changes were calculated by subtracting the mean of the first 10 data-points for each, the $\mathrm{x}-, \mathrm{y}$ - and $\mathrm{z}$-direction, and summarizing them by the square root of the sum of the squared three directions.

Outcomes focusing on temporal aspects of motion included movement time, speed, and smoothness metrics. Movement time was defined as the time between from movement onset and movement end. Peak velocity is determined by the maximum of hand velocity along the three directions with respect to the global reference frame, that were summarized by square root of the sum of the three directions. Similarly, the number of velocity peaks $(N V P)$, was summarized for all three directions, reflecting changes between acceleration and deceleration phases and thereby the smoothness of the movement profile. The NVP is applied dimensionless without a per time unit, and valid, however could lack sensitivity and reliability in case of measurement noise (Balasubramanian et al., 2015). Spectral arc length (SPARC) has been suggested for its robustness against measurement noise (Balasubramanian et al., 2015). SPARC was defined to reflect the spectral energy induced by unsmooth, saccadic motions (Balasubramanian et al., 2005).

\subsubsection{Classification of relevant movement phases}

As described in previous studies, segmentation of upper limb activities is important, to explore relevant aspects of task performance (Alt-Murphy et al., 2018; Repnik et al., 2019; Schwarz et al., 2020). For the purpose of this study, the movement primitives, reach distally to grasp or gesture and reaching proximally to transport or gesture, were further analyzed. As described by Schambra and colleagues (2019), reaches are defined by motions with the intention to make contact with an object or target that might include contact in terms of grasp or touch at the end of movement (Schambra et al., 2019). The motion primitive of transport is defined by the purpose to convey an object, that can result in motion away from the body or towards the body or head specifically. In the present study, relevant workspace directions were further differentiated, such as reaching ahead in ipsilateral distance within armlength and reaching towards the head. During a drinking task (T12, T14), taking the 
phone for a call (T13), biting into an apple (T15), putting on a hat (T16), or tooth brushing (T18), both phases of reach towards the object and transport towards the mouth, ear, or head are differentiable in terms of workspace related subphases. The phases of reach and transport were determined by grasp versus no grasp and manually selected since no information for grasp detection, such as by finger movements were applicable.

Semi-automated phase detection was used by feature-based movement detection algorithms as illustrated in Figure 5.3. Similarly, feature-based segmentation has been used based on finger force detection and finger angular motions in reach-to-grasp movements (Schwarz et al., 2020), as well as based on orientation angles of a body-worn sensor in ambulation assessments (Ortega-Bastidas et al., 2019). The primitives of reaching distally to grasp or gesture towards maximum ipsilateral arm length were determined based on the maximum distance in position in $\mathrm{x}$-direction along the sagittal plane and the velocity data of the handIMU. The primitives of reaching proximally to transport or gesture in direction towards the head were determined by the maximum position of the hand-IMU in $\mathrm{z}$-direction along gravity vector, as well as the minimum position of the hand-IMU in $\mathrm{x}$-direction.

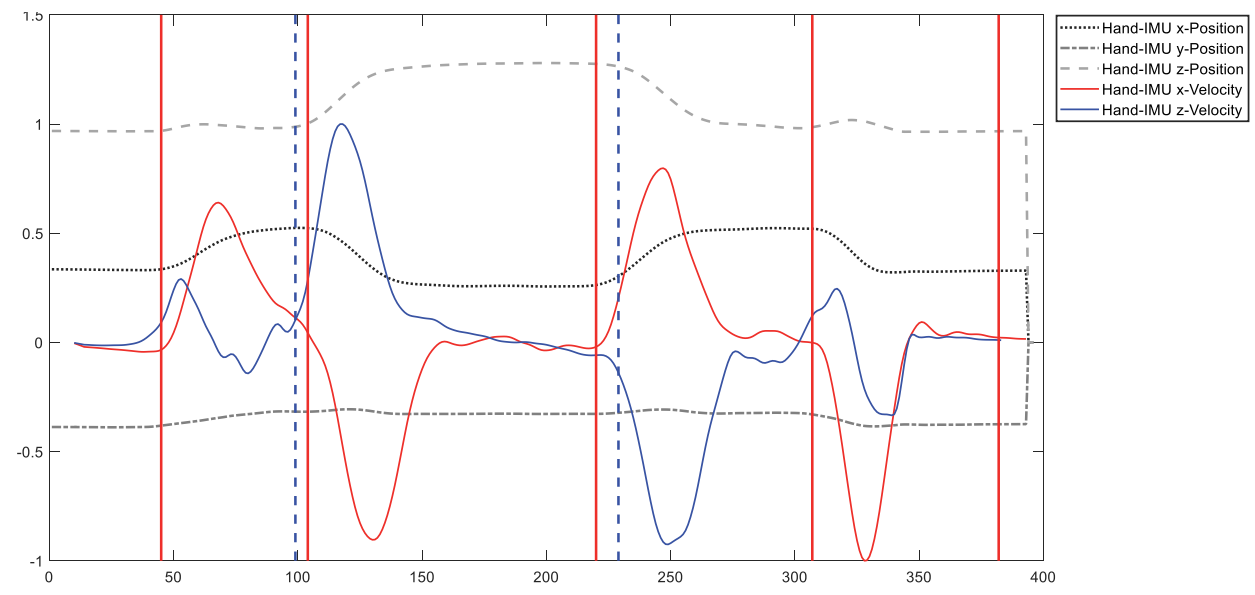

Figure 5.3. Feature-based movement phase segmentation.

Reaching or transporting distally towards maximum ipsilateral armlength is detected from movement onset to maximum distance of the hand-IMU in $\mathrm{x}$-direction, combined with a velocity threshold in $\mathrm{x}$ - and $\mathrm{z}$-direction. Reaching or transporting proximally towards the head was defined by the hand-IMU trajectory maximum height in $z$-direction combined with a velocity threshold in the $\mathrm{x}$ - and $\mathrm{z}$-direction. The automatic detection method focused on 
detecting the highest/lowest peaks in the velocity profile of the hand-IMU and identifying the beginning of increase in velocity according to a threshold in the angular velocity of 0.02 $\mathrm{rad} / \mathrm{s}$. The start of the reach corresponds to the first increase of the angular velocity of the hand IMU in the $\mathrm{x}$-axis. The next point corresponds to the increase in the angular velocity of the sensor in the $\mathrm{z}$ direction, indicating the beginning of the movement towards the head. The start of the 'Return Object' phase is identified by the increase of the angular velocity in the negative $z$-direction. Finally, the last phase is identified via the last negative peak of the angular velocity profile of the sensor's $x$-direction. The automatic phase-detection was verified manually on a trial-by-trial basis. Based on pre-assumptions of similar workspace, the phase segmentation was performed for the gesture movements T02, T05, T06, T07, T08, T10, and the grasp movements of the tasks T12, T13, T14, T15, T16, and T18. For gesture movement, only three points were determined, the movement onset, target position, as defined by maximum hand distance $\mathrm{x}$-direction and or maximum height in $\mathrm{z}$-direction, and the movement end.

\subsubsection{Statistical analysis}

The statistical analysis was performed by use of Matlab (MATLAB version 2018b, The Mathwork, Natick, MA) and SPSS (SPSS version 26.0, IBM Corp. Armonk, N.Y., USA). All kinematic outcome parameters were investigated for normal distribution by inspection of histograms. Descriptive statistics of the kinematic outcome parameters were presented in mean and the $95 \%$ confidence intervals.

For investigating impairment-based expressions in kinematic outcomes, the participants were allocated to the no, mild or moderate impairment group. The healthy subjects with a full score in the Fugl-Meyer Assessment of the Upper Extremity (FMA-UE) of 66 / 66 points constituted the no impairment group. Subjects with an FMA-UE score ranging 4865 / 66 points were assigned to the mild impairment group and those with an FMA-UE score ranging from 32-47 / 66 points to the moderate impairment group (Fugl-Meyer et al., 1975; Alt Murphy et al., 2011; Persson et al., 2015; Hoonhorst et al., 2015; Alt-Murphy et al., 2018). A linear mixed effects model analysis has been performed for each kinematic outcome parameter to test for the impact of the factor gesture versus grasping movements, and the factor of the impairment level as fixed effects.

To address the second research question on comparability of kinematic expression between motion primitives of reaching distally and proximally, we considered the kinematic values of the subphases of the tasks T02, T05, T06, T07, T08, T10, T12, T13, T14, T15, T16, and 
T18. Differences between metrics were tested for the factor of the movement task by use of the Kruskal-Wallis test, a non-parametric version of the one-factor ANOVA for reaching distally to grasp, reaching distally to gesture, reaching proximally to transport, or reaching proximally to gesture. Additionally, correlations between the ten core-set metrics were explored visually in a correlation matrix for each task sub phase. Correlation coefficients of $r \geq 0.5$ were defined as significant and compared across tasks.

\subsection{Results}

Thirty-one subjects were included in the present analysis, with the participant characteristics presented in Table 5.1. Fourteen subjects were affected on their dominant hand. Three subjects missed one movement item (S09 missed T03, S13 missed T09, and S23 missed T17)

Table 5.1. Participant characteristics

\begin{tabular}{|c|c|}
\hline Characteristic & Total $(\mathrm{N}=31)$ \\
\hline Stroke subjects in the chronic stage $>6$ months $(\mathrm{N})$ & 26 \\
\hline Gender, female/male & $9 / 17$ \\
\hline Mean age (SD), years & $62.19(12.10)$ \\
\hline Mean body height (SD), $\mathrm{cm}$ & $173.81(10.94)$ \\
\hline Mean BMI (SD), kg/m² & $26.97(4.23)$ \\
\hline Paretic body side, left/right & $13 / 13$ \\
\hline Months since stroke * & $20.50(12-34)$ \\
\hline Initial stroke severity NIHSS * & $8(6-11)$ \\
\hline $\operatorname{MoCA}(0-30) *$ & $27(24-28)$ \\
\hline MAS sum of the upper extremity $(0-14) *+$ & $1.75(0.25-3)$ \\
\hline EmNSA-UE $(0-40) *$ & $38(36-39)$ \\
\hline FMMA-UE $(0-66) *$ & $47.50(40.25-55.00)$ \\
\hline FMMA-UE arm subsection $(0-36) *$ & $26(22.00-29.75)$ \\
\hline FMMA-UE wrist subsection (0-10) * & $6(6.00-7.75)$ \\
\hline FMMA-UE hand subsection (0-14) * & $11(9.00-14.00)$ \\
\hline FMMA-UE coordination subsection (0-6) * & $4(3.25-5.00)$ \\
\hline Healthy subjects $(\mathrm{N})$ & 5 \\
\hline Gender, female/male & $2 / 3$ \\
\hline Mean age (SD), years & $65.75(10.72)$ \\
\hline Mean body height (SD), $\mathrm{cm}$ & $169.41(7.47)$ \\
\hline Mean BMI (SD), kg/m² & $23.26(2.18)$ \\
\hline
\end{tabular}

BMI, Body Mass Index; EmNSA, Erasmus modified version of the Nottingham Sensory Assessment; FMMAUE, Fugl-Meyer Motor Assessment of the Upper Extremity; MAS, modified Ashworth Scale; MoCA, Montreal Cognitive Assessment; NIHSS, National Institutes of Health Stroke Scale; L, left; SD, standard deviation. ${ }^{*}$ Indicates that values are presented in median (interquartile range); ${ }^{\dagger}$ Indicates MAS scores between 1 and 2 for seven muscle groups. 
of the 20 prescribed actions. The whole dataset consisted of 775 kinematic sets of the affected upper limb, represented by the mean values of the three repetitions per subject and task.

\subsubsection{Core-set kinematics of the total task execution}

As shown in Table 5.2, all kinematic parameters were statistically significant different between the gesture and grasp movements, when considering the total task execution across subjects. Gestures resulted in larger shoulder joint motions beside higher peak velocity when compared to the grasp movements. The grasp movements were associated with larger trunk motions, increases in elbow and wrist flexion/extension ranges and forearm pronation/supination, as well as an increase in the number of velocity peaks. On the level of subgroup comparisons between subjects with no, mild, and moderate impairments, spatial and temporal kinematics were included in the linear mixed model and illustrated in Figure 5.4. Significant effects of the impairment level were found for trunk displacement $(p=0.010)$, and shoulder flexion/extension ( $\mathrm{p}=0.001$ ), with statistically significant post-hoc comparison between no impairment and moderate impairment, as well as between mild and moderate impairment for both kinematic outcomes.

Significant effects were found for the interactions between the task and no impairment group on shoulder abduction/adduction $(\mathrm{p}=0.037)$. Movement time was significantly affected by the factor's impairment level $(\mathrm{p}<0.001)$, affected side is the dominant side $(\mathrm{p}=0.038)$, and the interaction between the task and the impairment level $(\mathrm{p}<0.001)$. Subjects of the no

Table 5.2. Linear mixed model results on kinematics for the factor movement task across subjects

\begin{tabular}{lllll}
\hline & All movements & $\begin{array}{l}\text { Gesture } \\
\text { movements }\end{array}$ & $\begin{array}{l}\text { Grasp } \\
\text { movements }\end{array}$ & Significance \\
\hline Trunk compensation in cm & $5.2(4.3-7.3)$ & $2.9(2.2-3.6)$ & $7.1(6.1-8.2)$ & $\mathrm{p}<0.001^{*}$ \\
Shoulder flex/ext in degrees & $52.1(45.0-60.2)$ & $76.1(70.3-81.8)$ & $66.5(62.4-70.6)$ & $\mathrm{p}<0.001^{*}$ \\
Shoulder abd/add in degrees & $17.9(15.7-24.0)$ & $26.7(24.3-29.3)$ & $29.3(27.5-31.0)$ & $\mathrm{p}=0.035^{*}$ \\
Elbow flex/ext in degrees & $55.7(46.3-63.8)$ & $71.3(65.3-77.4)$ & $92.33(89.0-95.6)$ & $\mathrm{p}<0.001^{*}$ \\
Forearm pro/sup in degrees & $42.5(31.5-54.8)$ & $54.1(49.1-59.1)$ & $65.5(58.2-66.9)$ & $\mathrm{p}=0.008^{*}$ \\
Wrist flex/ext in degrees & $33.3(23.6-41.7)$ & $21.1(19.2-23.0)$ & $33.2(30.0-36.3)$ & $\mathrm{p}<0.001^{*}$ \\
Movement time in seconds & $6.7(5.5-8.4)$ & $3.43(3.17-3.69)$ & $7.05(6.46-7.64)$ & $\mathrm{p}<0.001^{*}$ \\
Peak velocity in m/s & $0.8(0.8-1.0)$ & $1.99(1.81-2.18)$ & $1.22(1.13-1.31)$ & $\mathrm{p}<0.001^{*}$ \\
No of velocity peaks & $63.2(49.6-77.2)$ & $21.0(17.8-24.2)$ & $58.0(49.4-66.5)$ & $\mathrm{p}<0.001^{*}$ \\
SPARC & $-4.2(-4.9-(-3.8))$ & $-3.3(-3.5-(-3.1))$ & $-3.6(-3.8-(-3.4))$ & $\mathrm{p}=0.023^{*}$ \\
\hline
\end{tabular}

Kinematic parameters are represented by mean and ( $95 \%$ confidence interval) for the totally collected data and for the gesture and grasp activities separately. ${ }^{*}$ Indicates statistically significant differences $(p<0.05)$ between gesture and grasp kinematics. 

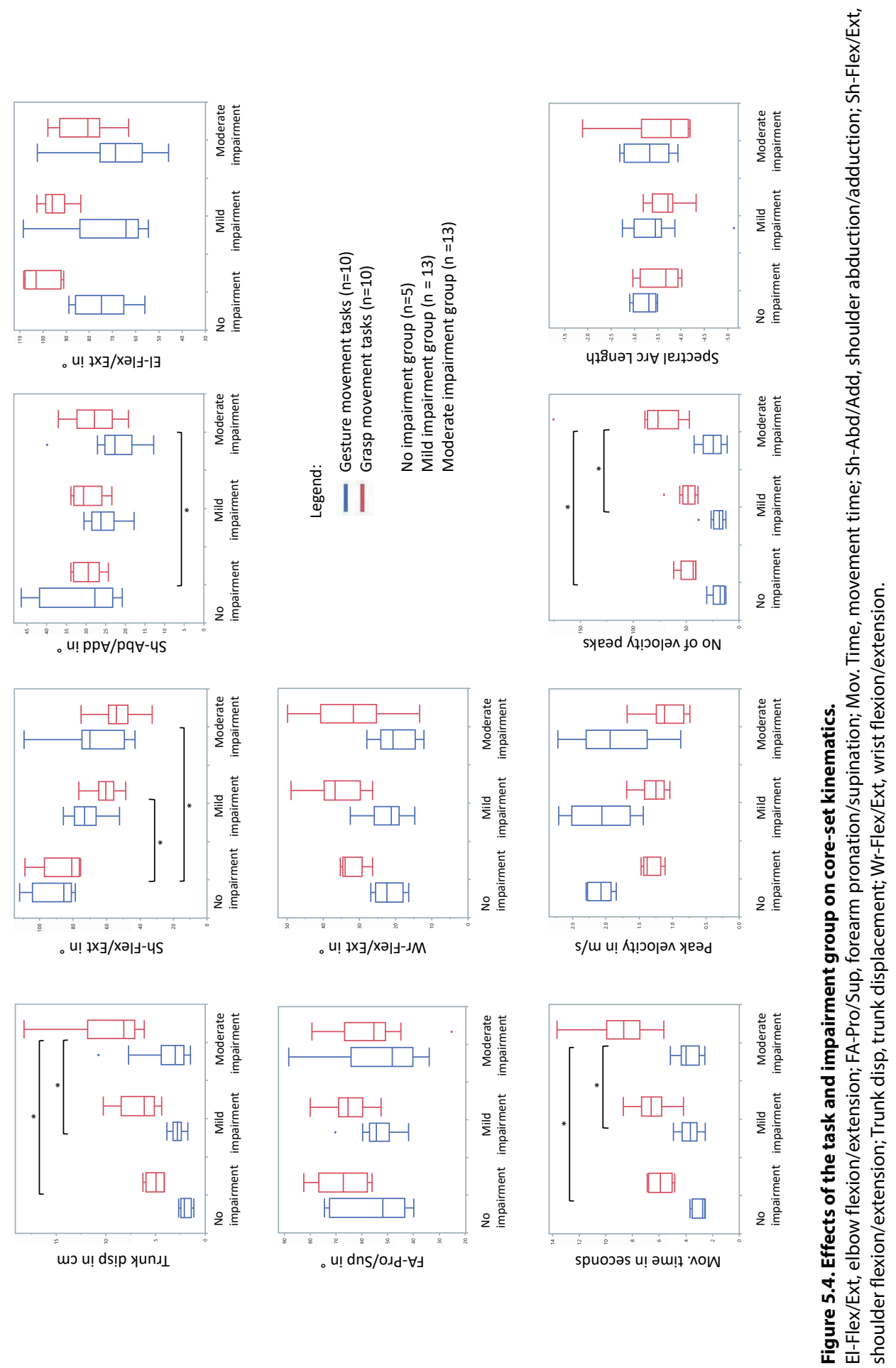
impairment group performed the tasks with a mean of 4.18 (3.30-5.06) seconds, compared to the mild impairment group with a mean of 5.16 (4.63-5.68) seconds and the moderate impairment group with a mean of 6.38 (5.85-6.90) seconds, which resulted in post-hoc significant differences between the no and moderate impairment group $(\mathrm{p}<0.001)$, and between the mild and moderate impairment group $(\mathrm{p}=0.006)$. NVP was shown to be influenced by the factor's impairment $(\mathrm{p}=0.001)$ and the interaction between the task and the impairment group ( $\mathrm{p}=0.002)$. Post-hoc comparisons for the impairment level revealed significant larger NVP's in the moderate impairment group with a mean of 53.0 (45.5-60.4) when compared to the no impairment group ( $\mathrm{p}=0.012)$ with a mean of $31.1(18.8-43.3)$ and the moderate impairment group $(\mathrm{p}=0.003)$ with a mean of $34.5(27.0-41.9)$.

\subsubsection{Core-set kinematics for sub phases of reaching proximally and distally with and without contact}

Four classes of sub phases, reaching distally to grasp, reaching distally to gesture, reaching proximally to transport, and reaching proximally to gesture, were predefined and detected in the tasks T02, T05, T06, T07, T08, T10, T12, T13, T14, T15, T16, and T18. Figure 5.5 illustrates the kinematic metrics per task and workspace given as median, upper, and lower quartile. The blue color code indicates movements distally towards the maximum reaching arm length while the green color code represents movements proximally towards the person's head. The one-factor analysis per kinematic parameter and the movement primitive classes resulted in statistically significant differences for the majority of conditions. For the factor of grasp movement tasks, the null hypothesis of comparable normal distributed outcomes per conditions could not be rejected for trunk displacement in reach distally $(\mathrm{p}=0.235)$ and transport proximally $(\mathrm{p}=0.413)$, for shoulder flexion/extension in reach distally $(\mathrm{p}=0.132)$, for shoulder abduction/adduction in reach distally $(\mathrm{p}=0.093)$, as well as for peak velocity in reach distally $(\mathrm{p}=0.108)$. For the gesture movement tasks, comparable results across tasks were found for shoulder flexion/extension in reach distally $(\mathrm{p}=0.613)$, for shoulder abduction in reach distally $(\mathrm{p}=0.104)$, for elbow flexion/extension in reach proximally $(\mathrm{p}=0.363)$, and for forearm pronation/supination in reach proximally $(\mathrm{p}=0.113)$. All temporal kinematics, such as movement time, peak velocity, number of velocity peaks and spectral arc length, were comparable between gestures with $p$-values ranging between $p=0.0866$ and $p=0.290$.

The relationship between the core-set kinematic were investigated in a correlation matrix for each task and motion sub phase. Figure 5.6 illustrates the strength of correlations across all subjects in a heatmap for reaching distally in blue color code and reaching proximally in 

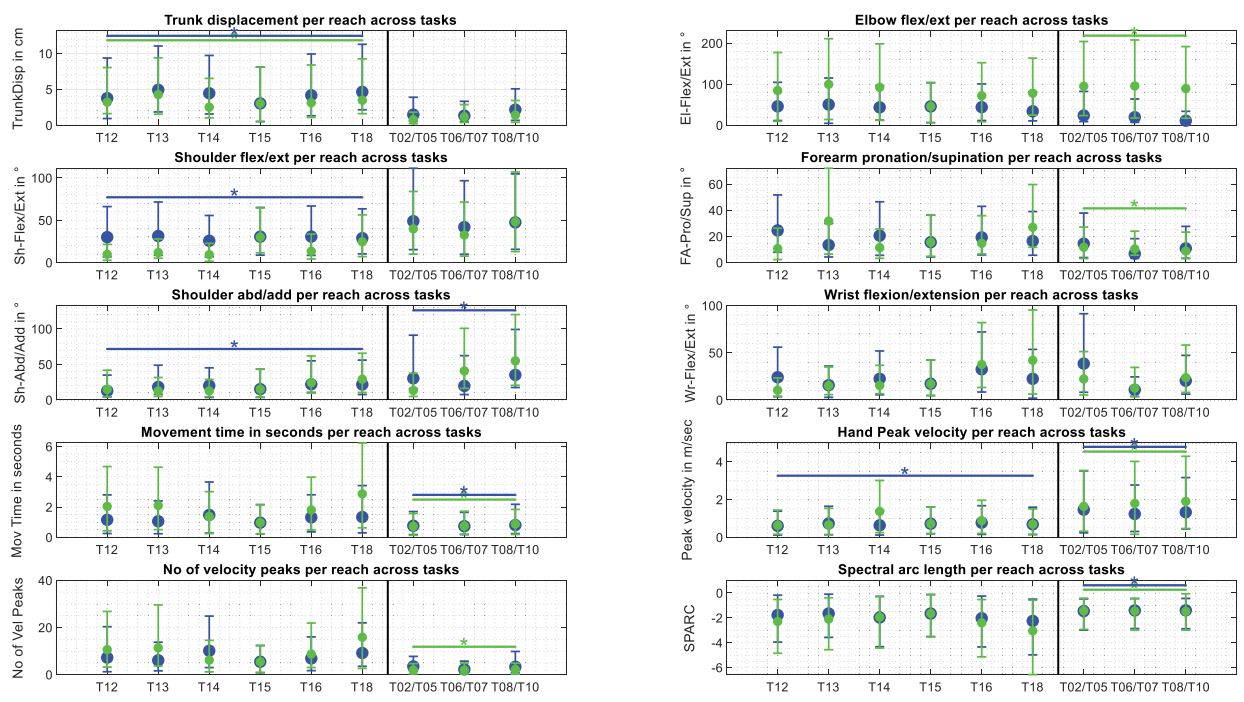

Figure 5.5. Kinematic metrics per movement reach sub phases across subjects.

El-Flex/Ext, elbow flexion/extension; FA-Pro/Sup, forearm pronation/supination; MovTime, movement time; No ofVelPeak, number of peak velocity; Sh-Abd/Add, shoulder abduction/adduction; Sh-Flex/Ext, shoulder flexion/ extension; SPARC, spectral arc length; TrunkDisp, trunk displacement; Wr-Flex/Ext, wrist flexion/extension. The metric values are presented per task across subjects by the median, the upper and lower quartile, with blue dots corresponding to reaching distally movements and green dots corresponding to reaching proximally. Statistically significant comparable metrics are indicated in the corresponding color code.

green color code. Correlation coefficients of $r \geq 0.5$, or $r \leq-0.5$ were set as cut-off to determine significant associations between metrics and circled by the black squares in the heatmap, in case of similar associations can be seen across tasks. During reach to grasp primitives, a stable relation between elbow and shoulder flexion/extension, between peak velocity and elbow and shoulder flexion/extension, between the number of velocity peaks and movement time, and between the number of velocity peaks and spectral arc length, has been shown. For reach to gesture, stable relations across tasks were found between peak velocity and shoulder flexion/ extension, between the number of velocity peaks and elbow flexion/extension, between the number of velocity peaks and movement time, and between the number of velocity peaks and the spectral arc length.

During proximal motion, stable relations across tasks were found between the number of velocity peaks and movement time. 

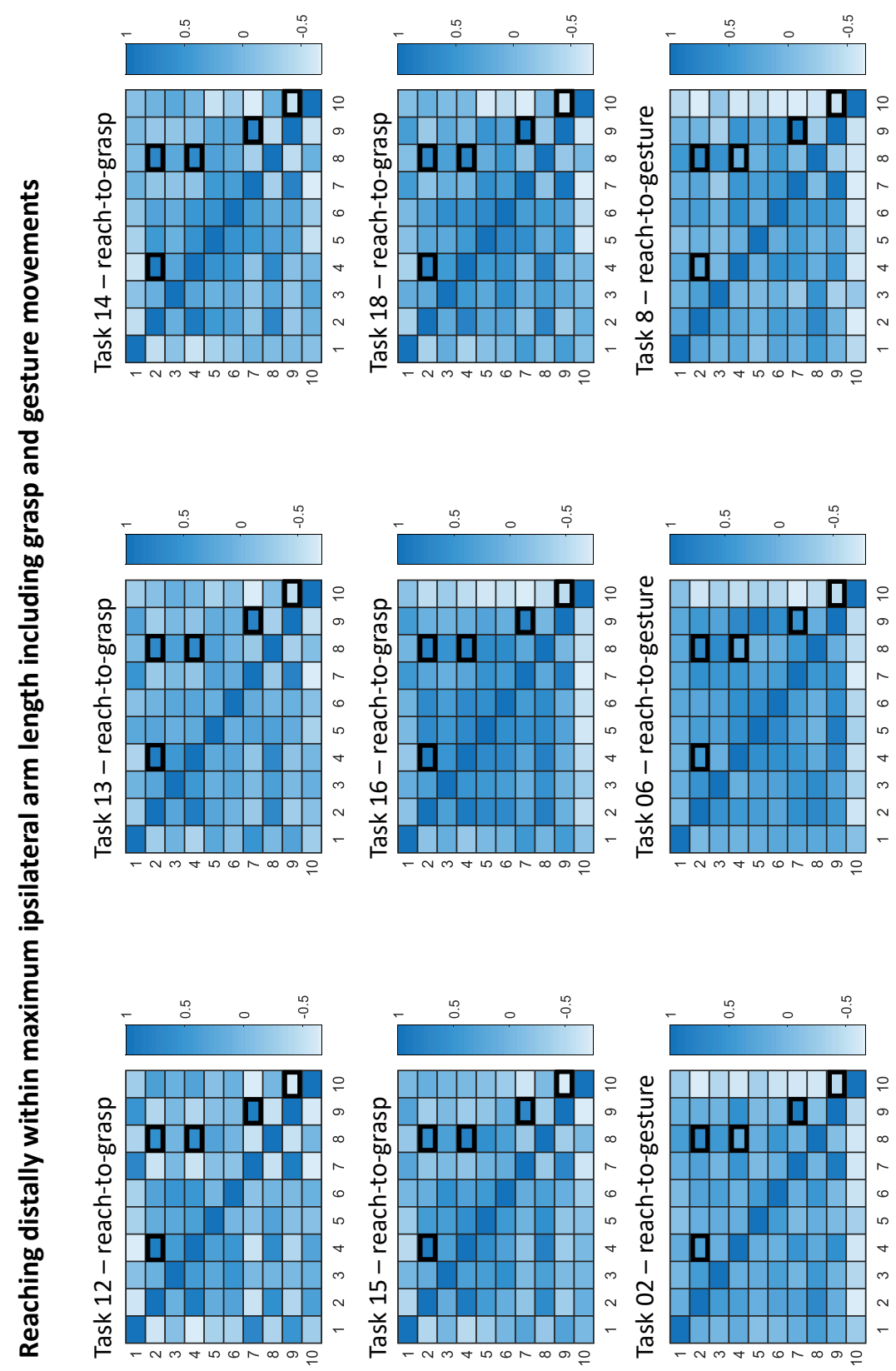

๖े

药 흥

3

등

들

空

ㅎ

은

흥

ह

ㅎํำ

in

¿

돌

졷

ن. $\frac{0}{x}$

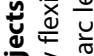

글 응

นิ $\overline{\mathrm{v}}$

o

든 는 눙

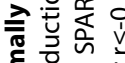

흦 융으

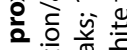

응

듬 응

층 는

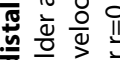

응 훙

次它

ฮ

to $\frac{2}{0}$

ป

\%

过

站

व

흥

उ

品占

을 눈

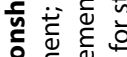

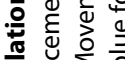

지일

응 응

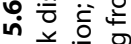

는

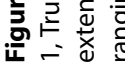



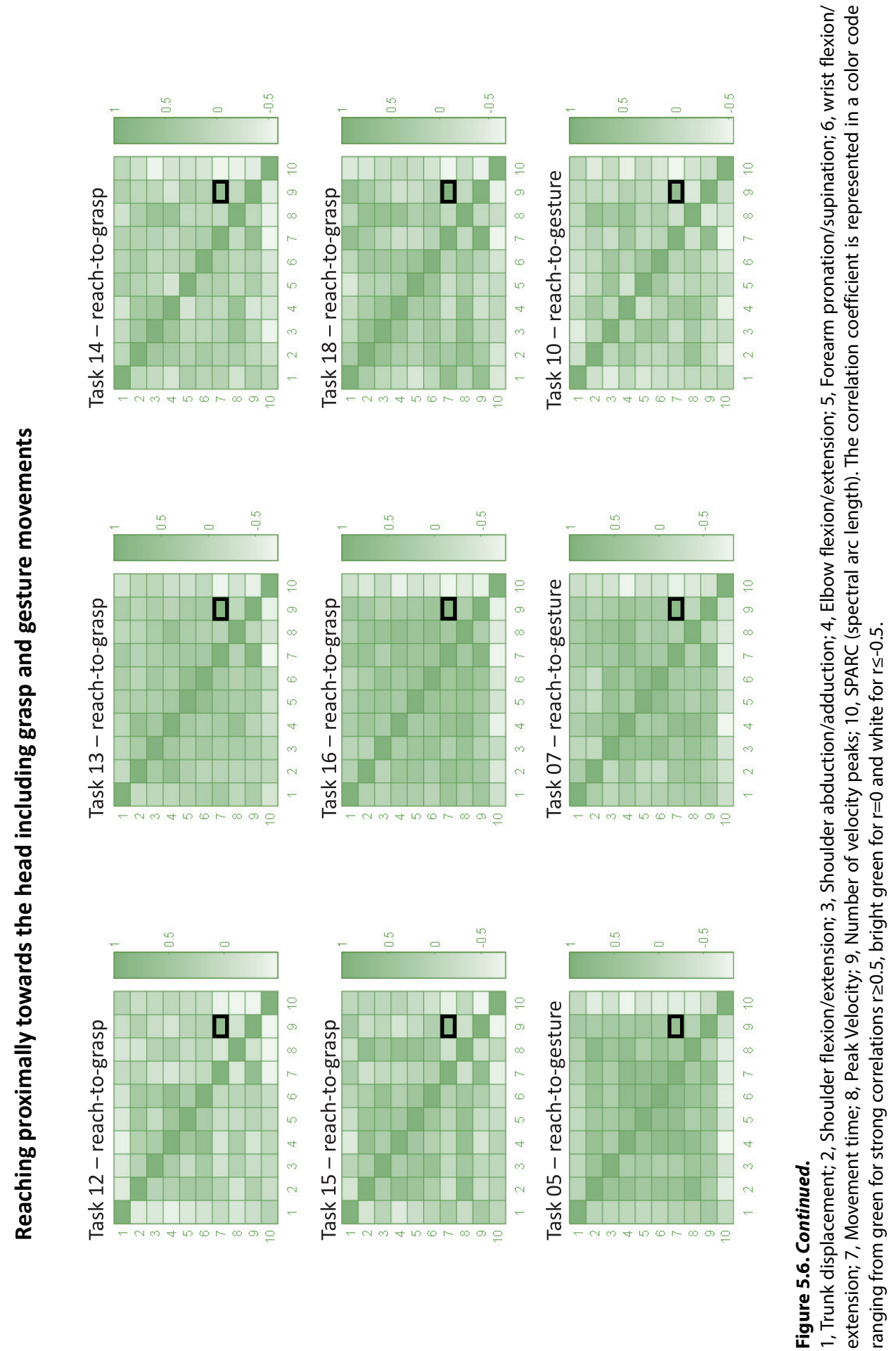


\subsection{Discussion}

In this study, it was aimed to characterize and compare movement quality measures based on a set of spatiotemporal kinematics in subjects with stroke-related upper limb impairments when performing various activities of daily living and with respect to their upper limb impairment level. Secondly, movement sub phases or primitives of reaching distally and reaching proximally were compared for all subjects across different tasks. Based on the presented results, task-specific expressions can be confirmed comparing a set of gesture movement without physical contact with the environment with a set of grasp and manipulation movements including contact to objects for the whole study sample. All kinematic parameters differed significantly between the movement tasks, with gesture movement were shown to be related to larger and faster movements, while grasp movements were related to larger trunk motions and joint ranges in forearm pronation/supination and wrist flexion/extension. The factor of no, mild and moderate impairment had significant effects on trunk displacement, shoulder flexion/extension, shoulder abduction/adduction, movement time and the number of velocity peaks. On the level of motion primitives, consistent relationships between shoulder, elbow flexion/extension, and peak velocity were shown in the primitive of reach to grasp distally and reach to gesture distally. Primitives of reach to transport or gesture proximally showed less significant correlations between the core-set metrics with the relation between the number of velocity peaks and movement time being the only consistent across tasks.

These results justify the importance of considering the task content, when aiming to quantify upper limb functions. The kinematic differences between gesture and grasp movements observed in this study are supported by the pioneering works of Marc Jeannerod (19352011), who found that the acceleration phase during reach-to-grasp is shorter compared to the deceleration phase in approach of the grasp, whereas during pointing movements the acceleration phase is considerably longer (Jeannerod, 1990). Furthermore, different tasks, such as rhythmic or discrete tasks were suspected to be controlled through different mechanisms (Hogan et al., 2007; Huys et al., 2008). The findings on spatiotemporal movement characteristics of moderate to mildly affected stroke subjects are in line with other findings on movement kinematics of ADL movements, such as the drinking task assessment (Alt Murphy et al., 2011), in terms of trunk compensation and peak velocity. Differences in kinematic measures in association with task difficulty were detected across impairment severities, in the less-affected upper limb, as well as in healthy subjects (Hussain 
et al., 2018, Poston et al., 2009) underpinning the importance of task characteristics in upper limb assessments.

Although the high task-dependency of the number of velocity peaks has been criticized in comparison to other smoothness measures, such as spectral arc length (Balasubramanian et al., 2015), the present study indicates good discriminability between overall upper limb impairment severity and limitation-levels in hand function. The strong and consistent relation between the number of velocity peaks and spectral arc length across reaching distally motion primitives supports the assumption that both measures reflect the movement construct of smoothness. Other studies suggested normalized jerk as a unitless measure that normalizes for both amplitude and duration of the movement (Buma et al., 2016) and log dimensionless jerk as appropriate for daily living recordings of different trial durations (Gulde et al., 2018).

This study supports the usefulness of quantification of movement quality during activities of daily living by use of a wearable sensing suit. Thus, spatiotemporal parameters were shown to be useful for assessing the total task performance as well as on the level of sub phases. The description of movements including gestures with no contact and grasp movements with object contact provides insights on possible standardized assessment protocols covering a main reduced core-set of upper limb functions. On the other hand, applications of real-life assessments could be thinkable. Schambra and colleagues validated their motion primitive taxonomy by kinematic recordings placed high-speed cameras and kinematic measures collected by nine IMUs placed on the pelvis, the thoracic and cervical spine, the upper arms, the lower arms, and the hands. The authors found that the two primitives of transport and reach were differentiable based on an unbiased machine learning algorithm with an accuracy of $92.1 \%$. The algorithm's nodes of the binary tree indicated greater wrist extension, wrist supination and elbow extension in reach, compared to less wrist extension and supination and more shoulder flexion and abduction during transport (Schambra et al., 2019), which is comparable to the results of the present study. Movement intention detection has been further investigated by use of hand and finger IMU's to ease the operating mode of hand orthosis (de Vries et al., 2018).

The detection of motion primitives, such as reach and transport with respect to the target positions in arm length reaching distance and towards the head or mouth, likely presents important prerequisites for relevant functioning in daily life. The fact that limitations in shoulder flexion/extension were detectable across tasks and in relation to the impairment- 
based subgroups supports the validity of this outcome parameter in upper limb assessments after stroke. The limitations in joint range could be related to weakness, limited muscle activation towards the limits of end range or related to soft-tissue changes, since all included subjects were at the chronic stage where stroke-related limitations tend to be manifested. Besides the informativeness for upper limb kinematic assessments after stroke, the herein presented findings in diverting kinematics with respect to the impairment's severity might also lead to new specific targets for treatment approaches, including training concepts and developments in assistive devices and technology. Questions like, does specific training of the shoulder muscles, especially the rotator cuff or assistive devices such as the iron arm improve functionality and quality of movement kinematics after stroke, would be interesting to be addressed in future studies.

Limitations of the study relate to the restriction on kinematic measures without taking into account kinetics, such as muscle activity or force detection. For the phase detection of motion primitives across movement items, the presented study did not include a comparable data source, such as video recordings or interaction force detection. Also, no reference for kinematic outcomes, joint angle and position estimates. We have not included analysis of the dominant versus the non-dominant hand. The factor of the affected is the dominantside has been included in the linear-mixed model, without revealing significant effects on one of the tested kinematic parameters.

\subsection{Conclusion}

In conclusion, the analysis of gesture and grasp movements in a set of daily living activities revealed task-specific and impairment-specific characteristics in terms of differences in spatial and temporal kinematic parameters. Grasping motion was characterized by more distal pronounced and slower motions, that were less smooth and executed with larger trunk motions in stroke subjects, when compared to faster gesture movement, that were shown to be less discriminative between impairment levels. In that, it could be demonstrated that kinematic assessments of activities of daily living provide general and granular information on movement quality of relevant and natural activities. Semi-automatic phase detection analysis of motion primitives enabled analysis of movement quality and the comparability of the primitives across various activities. 


\section{Funding}

This project has received funding from the European Union's Horizon 2020 Research and Innovation Programme under grant agreement number 688857 (SoftPro), from the Swiss State Secretariat for Education, Research and Innovation (contract number 15.0283-1), and the P\&K Pühringer Foundation.

\subsection{References}

Alt Murphy M, Häger CK. Kinematic analysis of the upper extremity after stroke-how far have we reached and what have we grasped? Phys Ther Rev. 2015;20:137-55.

Alt Murphy M, Murphy S, Persson HC, Bergström UB, Sunnerhagen KS. Kinematic Analysis Using 3D Motion Capture of Drinking Task in People With and Without Upper-extremity Impairments. J Vis Exp. 2018;133:e57228. doi: 10.3791/57228

Alt Murphy M, Willen C, Sunnerhagen KS. Kinematic variables quantifying upper-extremity performance after stroke during reaching and drinking from a glass. Neurorehabil Neural Repair. 2011;25:71-80.

Averta G, Della Santina E, Battaglia E, Felici F, Bianchi M, Bicchi A. Unvealing the principal modes of human upper limb movements through functional analysis. Front Robot AI. 2017;4:37.

Averta G, Della Santina C, Valenza G, Bicchi A, Bianchi M. Exploiting upper-limb functional principal components for human-like motion generation of anthropomorphic robots. J Neuroeng Rehabil. 2020;17:63. doi: 10.1186/s12984-020-00680-8

Balasubramanian S, Melendez-Calderon A, Roby-Brami A, Burdet E. On the analysis of movement smoothness. J NeuroEng Rehabil. 2015;12:112. doi: 10.1186/s12984-015-0090-9

Bang DH, Shin WS, Choi SJ. The effects of modified constraint-induced movement therapy combined with trunk restraint in subacute stroke: A double-blinded randomized controlled trial. Clin Rehabil. 2015;29:561-9.

Bartolo M, De Nunzio AM, Sebastiano F, et al. Arm weight support training improves functional motor outcome and movement smoothness after stroke. Funct Neurol. 2014;29:15-21.

Bensmail D, Robertson JVG, Fermanian C, Roby-Brami A. Botulinum toxin to treat upper-limb spasticity in hemiparetic patients: Analysis of function and kinematics of reaching movements. Neurorehabil Neural Repair. 2010b;24:273-81.d

Buma FE, van Kordelaar J, Raemaekers M, van Wegen EEH, Ramsey NF, Kwakkel G. Brain activation is related to smoothness of upper limb movements after stroke. Exp Brain Res. 2016;234:2077-89. doi: $10.1007 / \mathrm{s} 00221-015-4538-8$

Colombo R, Pisano F, Delconte C, et al. Comparison of exercise training effect with different robotic devices for upper limb rehabilitation: A retrospective study. Eur J Phys Rehabil Med. 2017;53:2408.

Conrad MO, Gadhoke B, Scheidt RA, Schmit BD. Effect of tendon vibration on hemiparetic arm stability in unstable workspaces. PLoS One. 2015a;10:e0144377.

Corti M, McGuirk TE, Wu SS, Patten C. Differential effects of power training versus functional task practice on compensation and restoration of arm function after stroke. Neurorehabil Neural Repair. 2012;26:842-54. 
Cutkosky MR. On grasp choice, grasp models, and the design of hands for manufacturing tasks. IEEE Trans Robot. 2007;5:269-79.

de Vries J, van Ommeren A, Prange-Lasonder G, Rietman J, Veltink P. Detection of the intention to grasp during reach movements. J Rehabil Assist Technol Eng. 2018;5:2055668317752850. doi: $10.1177 / 2055668317752850$

Di Lazzaro V, Capone F, Di Pino G, et al. Combining robotic training and non-invasive brain stimulation in severe upper limb-impaired chronic stroke patients. Front Neurosci. 2016;10:88.

Durham KF, Sackley CM, Wright CC, Wing AM, Edwards MG, van Vliet P. Attentional focus of feedback for improving performance of reach-to-grasp after stroke: A randomised crossover study. Physiotherapy. 2014;100:108-15.

Ellis MD, Carmona C, Drogos J, Traxel S, Dewald JP. Progressive abduction loading therapy targeting flexion synergy to regain reaching function in chronic stroke: Preliminary results from an RCT. Ann Int Con IEEE Eng Med Biol Soc. 2016;2016:5837-40.

Feix T, Romero J, Schmiedmayer HB, Dollar AM, Kragic D. The grasp taxonomy of human grasp types. IEEE Trans Hum Mach Syst. 2016;46:66-77.

Fugl-Meyer AR, Jääskö L, Leyman I, Olsson S, Steglind S. The post-stroke hemiplegic patient. 1. a method for evaluation of physical performance. Scan J Rehabil Med 1975;7:13-31.

Giszter SF. Motor primitives--new data and future questions. Curr Opin Neurobiol. 2015;33:156-65. doi: 10.1016/j.conb.2015.04.004

Gulde P, Hermsdörfer J. Smoothness Metrics in Complex Movement Tasks. Front Neurol. 2018;9:615. doi: 10.3389/fneur.2018.00615

Held JPO, Klaassen B, Eenhoorn A, van Beijnum BF, Buurke JH, Veltink PH, Luft AR. Inertial Sensor Measurements of Upper-Limb Kinematics in Stroke Patients in Clinic and Home Environment. Front Bioeng Biotechnol. 2018;6:27. doi: 10.3389/fbioe.2018.00027Roetenberg 2007

Hogan N, Sternad D. On rhythmic and discrete movements: reflections, definitions and implications for motor control. Exp Brain Res. 2007;181:13-30. doi: 10.1007/s00221-007-0899-y

Hoonhorst MH, Nijland RH, van den Berg JS, Emmelot CH, Kollen BJ, Kwakkel G. How Do FuglMeyer Arm Motor Scores Relate to Dexterity According to the Action Research Arm Test at 6 Months Poststroke?. Arch Phys Med Rehabil. 2015;96:1845-9. doi: 10.1016/j.apmr.2015.06.009

Housman SJ, Scott KM, Reinkensmeyer DJ. A randomized controlled trial of gravity-supported, computer-enhanced arm exercise for individuals with severe hemiparesis. Neurorehabil Neural Repair. 2009;23:505-14.

Hsieh YW, Liing RJ, Lin KC, et al. Sequencing bilateral robot-assisted arm therapy and constraintinduced therapy improves reach to press and trunk kinematics in patients with stroke. J Neuroeng Rehabil. 2016;13:31.

Hussain N, Alt Murphy M, Sunnerhagen KS. Upper Limb Kinematics in Stroke and Healthy Controls Using Target-to-Target Task in Virtual Reality. Front Neurol. 2018;9:300. doi: 10.3389/ fneur.2018.00300

Huys R, Studenka BE, Rheaume NL, Zelaznik HN, Jirsa VK. Distinct timing mechanisms produce discrete and continuous movements. PLoS Comput Biol. 2008;4:e1000061. doi: 10.1371/journal. pcbi. 1000061

Jeannerod M. The neural and behavioural organization of goal-directed movements. Clarendon Press, Oxford; 1988. 
Kim H, Miller LM, Fedulow I, et al. Kinematic data analysis for post-stroke patients following bilateral versus unilateral rehabilitation with an upper limb wearable robotic system. IEEE Trans Neural Syst Rehabil Eng. 2013;21:153-64.

Klaassen B, van Beijnum BF, Held JP, Reenalda J, van Meulen FB, Veltink PH, Hermens HJ. Usability Evaluations of a Wearable Inertial Sensing System and Quality of Movement Metrics for Stroke Survivors by Care Professionals. Front Bioeng Biotechnol. 2017;5:20. doi: 10.3389/ fbioe.2017.00020

Kwakkel G, Lannin NA, Borschmann K, et al. Standardized measurement of sensorimotor recovery in stroke trials: Consensus-based core recommendations from the Stroke Recovery and Rehabilitation Roundtable. Int J Stroke. 2017;12(5):451-61. doi: 10.1177/1747493017711813

Lima RCM, Michaelsen SM, Nascimento LR, Polese JC, Pereira ND, Teixeira-Salmela LF. Addition of trunk restraint to home-based modified constraint-induced movement therapy does not bring additional benefits in chronic stroke individuals with mild and moderate upper limb impairments: A pilot randomized controlled trial. NeuroRehabilitation. 2014;35:391-404.

Lin KC, Chen YA, Chen CL, Wu CY, Chang YF. The effects of bilateral arm training on motor control and functional performance in chronic stroke: A randomized controlled study. Neurorehabil Neural Repair. 2010;24:42-51.

Massie C, Malcolm MP, Greene D, Thaut M. The effects of constraint-induced therapy on kinematic outcomes and compensatory movement patterns: An exploratory study. Arch Phys Med Rehabil. 2009;90:571-9

Mazzoleni S, Sale P, Tiboni M, Franceschini M, Carrozza MC, Posteraro F. Upper limb robot-assisted therapy in chronic and subacute stroke patients. Am J Phys Med Rehabil. 2013c:e26-37.

McCrea PH, Eng JJ, Hodgson AJ. Biomechanics of reaching: clinical implications for individuals with acquired brain injury. Disabil Rehabil. 2002;24:534-41.

Ortega-Bastidas P, Aqueveque P, Gómez B, Saavedra F, Cano-de-la-Cuerda R. Use of a Single Wireless IMU for the Segmentation and Automatic Analysis of Activities Performed in the 3-m Timed Up \& Go Test. Sensors (Basel). 2019;19:1647. doi: 10.3390/s19071647

Paulich M., Schepers M., Rudigkeit N., Bellusci G. Xsens MTw Awinda: Miniature WirelessInertialMagnetic Motion Tracker for HighlyAccurate 3D Kinematic Applications. https://www.xsens. com/hubfs/3446270/Downloads/Manuals/MTwAwinda_WhitePaper.pdf

Persson HC, Alt Murphy M, Danielsson A, Lundgren-Nilsson Å, Sunnerhagen KS. A cohort study investigating a simple, early assessment to predict upper extremity function after stroke - a part of the SALGOT study. BMC Neurol. 2015;15:92. doi: 10.1186/s12883-015-0349-6

Piron L, Turolla A, Agostini M, et al. Motor learning principles for rehabilitation: A pilot randomized controlled study in poststroke patients. Neurorehabil Neural Repair. 2010;24:501-8.

Poston B, Van Gemmert AW, Barduson B, Stelmach GE. Movement structure in young and elderly adults during goal-directed movements of the left and right arm. Brain Cogn. 2009;69:30-8. doi: 10.1016/j.bandc.2008.05.002

Quattrocchi G, Greenwood R, Rothwell JC, Galea JM, Bestmann S. Reward and punishment enhance motor adaptation in stroke. J Neurol Neurosurg Psychiatry. 2017;88:730-6.

Raghavan P. Upper Limb Motor Impairment After Stroke. Phys Med Rehabil Clin N Am. 2015;26:599610. doi: 10.1016/j.pmr.2015.06.008 
Repnik E, Puh U, Goljar N, Munih M, Mihelj M. Using Inertial Measurement Units and Electromyography to Quantify Movement during Action Research Arm Test Execution. Sensors (Basel). 2018;18:2767. doi: 10.3390/s18092767

Roetenberg D, Baten CT, Veltink PH. Estimating body segment orientation by applying inertial and magnetic sensing near ferromagnetic materials. IEEE Trans Neural Syst Rehabil Eng. 2007;15:46971. doi: 10.1109/TNSRE.2007.903946

Schambra HM, Parnandi A, Pandit NG, Uddin J, Wirtanen A, Nilsen DM. A Taxonomy of Functional Upper Extremity Motion. Front Neurol. 2019;10:857. doi: 10.3389/fneur.2019.00857

Schwarz A, Averta G, Veerbeek JM, Luft AR, Held JPO, Valenza G, Bicchi A, Bianchi M. A functional analysis-based approach to quantify upper limb impairment level in chronic stroke patients: a pilot study. Annu Int Conf IEEE Eng Med Biol Soc. 2019;2019:4198-204. doi: 10.1109/ EMBC.2019.8857732

Schwarz A, Bhagubai MMC, Wolterink G, Held JPO, Luft AR, Veltink PH. Assessment of Upper Limb Movement Impairments after Stroke Using Wearable Inertial Sensing. Sensors (Basel). 2020;20:4770. doi: 10.3390/s20174770

Schwarz A, Kanzler CM, Lambercy O, Luft AR, Veerbeek JM. Systematic Review on Kinematic Assessments of Upper Limb Movements After Stroke. Stroke. 2019;50:718-27. doi: 10.1161/ STROKEAHA.118.023531

Subramanian SK, Lourenco CB, Chilingaryan G, Sveistrup H, Levin MF. Arm motor recovery using a virtual reality intervention in chronic stroke: Randomized control trial. Neurorehabil Neural Repair. 2013;27:13-23.

Subramanian SK, Yamanaka J, Chilingaryan G, Levin MF. Validity of movement pattern kinematics as measures of arm motor impairment poststroke. Stroke. 2010;41:2303-8.

Van Delden AEQ, Beek PJ, Roerdink M, Kwakkel G, Peper CE. Unilateral and bilateral upperlimb training interventions after stroke have similar effects on bimanual coupling strength. Neurorehabil Neural Repair. 2015;29:255-67.

van Meulen FB, Klaassen B, Held J, Reenalda J, Buurke JH, van Beijnum BF, Luft A, Veltink PH. Objective Evaluation of the Quality of Movement in Daily Life after Stroke. Front Bioeng Biotechnol. 2016;3:210. doi: 10.3389/fbioe.2015.00210

van Meulen FB, Reenalda J, Buurke JH, Veltink PH. Assessment of daily-life reaching performance after stroke. Ann Biomed Eng. 2015;43:478-86.

Wu CY, Chen YA, Chen HC, Lin KC, Yeh IL. Pilot trial of distributed constraint-induced therapy with trunk restraint to improve poststroke reach to grasp and trunk kinematics. Neurorehabil Neural Repair. 2012a;26:247-55.

Wu CY, Chen CL, Tang SF, Lin KC, Huang YY. Kinematic and clinical analyses of upper-extremity movements after constraint-induced movement therapy in patients with stroke: A randomized controlled trial. Arch Phys Med Rehabil. 2007a;88:964-70.

Wu CY, Yang CL, Chen MD, Lin KC, Wu LL. Unilateral versus bilateral robot-assisted rehabilitation on arm-trunk control and functions post stroke: A randomized controlled trial. J Neuroeng Rehabil. 2013a;10:35.

Wu CY, Yang CL, Chuang LL, et al. Effect of therapist-based versus robot-assisted bilateral arm training on motor control, functional performance, and quality of life after chronic stroke: A clinical trial. Phys Ther. 2012c;92:1006-16. 
Wu C-Y, Huang P-C, Chen Y-T, Lin K-C, Yang H-W. Effects of mirror therapy on motor and sensory recovery in chronic stroke: A randomized controlled trial. Arch Phys Med Rehabil. 2013b;94:1023-30.

Xsene MVN User Manual, User Guide Xsens MVN, MVN Link, MVN Awinda, Document 760 MV0319P, Revision X, Oct 2018 Xsens, Enschede, NL, Accessed November 2018. 


\subsection{Supplementary material}

\section{Supplementary Table S5.1. Set of daily living tasks}

\begin{tabular}{|c|c|c|c|}
\hline$\#$ & Motion description & Contact & Primitives \\
\hline 1 & OK gesture & No & Move to $\mathrm{H}$, Reposition \\
\hline 2 & Thumb down (lifting) & No & Move to D, Reposition \\
\hline \multirow[t]{2}{*}{3} & $\begin{array}{l}\text { Exultation (extending the arm up in the air and keeping it in } \\
\text { with closed fist) }\end{array}$ & No & Move to D, Reposition \\
\hline & $\begin{array}{l}\text { Hitchhiking (extended elbow along the frontal plane, closed } \\
\text { fist, thumb up) }\end{array}$ & No & Move to E, Reposition \\
\hline 5 & $\begin{array}{l}\text { Block out sun from own face (with open hand, touch the } \\
\text { face covering the eyes) }\end{array}$ & No & Move to $\mathrm{H}$, Reposition \\
\hline 6 & Greet (with open hand, moving wrist) (3 times) & No & Move to D, Reposition \\
\hline 7 & Military salute (with lifted elbow) & No & Move to $\mathrm{H}$, Reposition \\
\hline 8 & $\begin{array}{l}\text { Stop gesture (extending the arm along the sagittal plane, } \\
\text { parallel to the floor, with extended elbow, open palm) }\end{array}$ & No & Move to D, Reposition \\
\hline 9 & $\begin{array}{l}\text { Pointing (with index finger) of something straight ahead } \\
\text { (with outstretched arm) }\end{array}$ & No & Move to D, Reposition \\
\hline 10 & $\begin{array}{l}\text { Silence gesture (bringing the index finger, with the } \\
\text { remainder of the hand closed, on the lips) }\end{array}$ & No & Move to $\mathrm{H}$, Reposition \\
\hline 11 & $\begin{array}{l}\text { each and grasp a small suitcase (placed along own frontal } \\
\text { plane) from the handle, lift it and place it on the floor (close } \\
\text { to own chair, along own sagittal plane) }\end{array}$ & No & $\begin{array}{l}\text { Reach, Transport E, } \\
\text { Reposition, Return }\end{array}$ \\
\hline 12 & $\begin{array}{l}\text { Reach and grasp a glass, drink for } 3 \mathrm{sec} \text {. and place it in the } \\
\text { initial position }\end{array}$ & $\begin{array}{l}\text { Yes } \\
* 3\end{array}$ & $\begin{array}{l}\text { Reach, Transport H, } \\
\text { Reposition, Return }\end{array}$ \\
\hline 13 & $\begin{array}{l}\text { Reach and grasp a phone receiver (placed along own sagittal } \\
\text { plane), carry it to own ear for } 3 \mathrm{sec} \text {. and place back }\end{array}$ & $\begin{array}{l}\text { Yes } \\
* 4\end{array}$ & $\begin{array}{l}\text { Reach, Transport H, } \\
\text { Reposition, Return }\end{array}$ \\
\hline 14 & $\begin{array}{l}\text { Reach and grasp a small cup from the handle ( } 2 \text { fingers + } \\
\text { thumb), drink for } 3 \mathrm{sec} \text {. and place it in the initial position }\end{array}$ & $\begin{array}{l}\text { Yes } \\
* 8\end{array}$ & $\begin{array}{l}\text { Reach, Transport H, } \\
\text { Reposition, Return }\end{array}$ \\
\hline 15 & $\begin{array}{l}\text { Reach and grasp an apple, mimic biting and put it in the } \\
\text { initial position }\end{array}$ & $\begin{array}{l}\text { Yes } \\
* 11\end{array}$ & $\begin{array}{l}\text { Reach, Transport H, } \\
\text { Reposition, Return }\end{array}$ \\
\hline 16 & $\begin{array}{l}\text { Reach and grasp a hat (placed on the right side of the table) } \\
\text { from its top and place it on own head }\end{array}$ & $\begin{array}{l}\text { Yes } \\
* 12,13\end{array}$ & $\begin{array}{l}\text { Reach, Transport H, } \\
\text { Reposition, Return }\end{array}$ \\
\hline 17 & $\begin{array}{l}\text { Reach and grasp a tennis racket (placed along own frontal } \\
\text { plane) and play a forehand (the subject is still seated) }\end{array}$ & $\begin{array}{l}\text { Yes } \\
* 2,3,4\end{array}$ & $\begin{array}{l}\text { Reach, Transport D, } \\
\text { Reposition, Return }\end{array}$ \\
\hline 18 & $\begin{array}{l}\text { Reach and grasp a toothbrush, brush teeth, and put the } \\
\text { toothbrush inside a cylindrical holder }\end{array}$ & $\begin{array}{l}\text { Yes } \\
* 5\end{array}$ & $\begin{array}{l}\text { Reach, Transport H, } \\
\text { Reposition, Return }\end{array}$ \\
\hline 19 & $\begin{array}{l}\text { Reach and grasp a laptop and open the laptop (without } \\
\text { changing its position) ( } 4 \text { fingers }+ \text { thumb) }\end{array}$ & $\begin{array}{l}\text { Yes } \\
* 6\end{array}$ & $\begin{array}{l}\text { Reach, Transport C, } \\
\text { Reposition, Return }\end{array}$ \\
\hline 20 & $\begin{array}{l}\text { Reach and grasp a doorknob (disk shape), turn it clockwise } \\
\text { and counterclockwise }\end{array}$ & $\begin{array}{l}\text { Yes } \\
* 10\end{array}$ & $\begin{array}{l}\text { Reach, Transport D, } \\
\text { Return }\end{array}$ \\
\hline
\end{tabular}

$C$, center position in front of the subject; $D$, distal position in ipsilateral arm length position; $E$, external position in abducted position parallel to the frontal plane; $\mathrm{H}$, head position including movements towards the mouth, ear, and top of head. * Indicated the grasp type as classified by Cutcowski (1989). 



\title{
Assessment of upper limb movement impairments after stroke using wearable inertial sensing
}

\author{
A. Schwarz \\ M.M.C. Bhagubai \\ G. Wolterink \\ J.P.O. Held \\ A.R. Luft \\ P.H. Veltink
}




\subsection{Abstract}

Precise and objective assessments of upper limb movement quality after strokes in functional task conditions are an important prerequisite to improve understanding of the pathophysiology of movement deficits and to prove the effectiveness of interventions. Herein, a wearable inertial sensing system was used to capture movements from the fingers to the trunk in 10 chronic stroke subjects when performing reach-to-grasp activities with the affected and non-affected upper limb. It was investigated whether the factors, tested arm, object weight, and target height, affect the expressions of range of motion in trunk compensation and flexion-extension of the elbow, wrist, and finger during object displacement. The relationship between these metrics and clinically measured impairment was explored. Nine subjects were included in the analysis, as one had to be excluded due to defective data. The tested arm and target height showed strong effects on all metrics, while an increased object weight showed effects on trunk compensation. High inter- and intrasubject variability was found in all metrics without clear relationships to clinical measures. Relating all metrics to each other resulted in significant negative correlations between trunk compensation and elbow flexion-extension in the affected arm. The findings support the clinical usability of sensor-based motion analysis.

Keywords - Upper extremity; stroke; biomechanical phenomena; kinematics; inertial measurement systems; motion analysis. 


\subsection{Introduction}

Human hand and arm function contribute to a wide range of activities in daily life, ranging from sensory functions to interacting with the environment and to functions that have a strong motor component like the manipulation of objects in grasping (Jones and Lederman, 2006). Hand and arm functionalities including object manipulation and physical interactions with the environment rely on the ability to control prehensile finger forces to perform specific grasp types (Parry et al., 2019; Feix et al., 2016) and ability to control both the distal and proximal joints of the upper limb in a goal-directed manner (Bernstein, 1967), for example when transporting the hand to reach the location of a desired object and forming the fingers for grasping (Jeannerod, 1994).

In subjects, experiencing upper limb impairments due to a stroke, these complex hand-and arm-grasping functionalities are defective (Ekstrand, 2016). Stroke is known as the leading cause of disability in the world (Vos et al., 2016), defined as a disruption in brain cell perfusion that leads to cell death and losses in network connectivity and multimodal impairments (Sacco et al., 2013). In particular, infarctions of the middle cerebral artery affecting the primary motor cortex and the integrity of the corticospinal tract have been associated with upper limb movement deficits (Byblow et al., 2015; Stinear et al., 2007), such as weakness, decreased interjoint coordination and in particular diminished finger dexterity (Raghavan, 2015; Santello and Lang, 2015). Of these motor performance aspects, weakness caused by stroke indicates the inability to activate certain upper limb muscles or segments, whereas interjoint coordination is defined as the ability to control all upper limb joints or segments in a spatially and temporally efficient manner. The differentiation between weakness and interjoint coordination during upper limb activities is rather precisely definable, as both show strong associations with each other (Sukal-Moulton et al., 2007) and other strokerelated impairments, such as spasticity (Levin, 1996). Phenotypes of stroke-related interjoint coordination deficits include the appearance of the pathological flexor synergy in reaching increased trunk movements to compensate for the upper limb limitations and a decreased finger dexterity for prehensile grasp application. The pathological flexor synergy was defined as a stereotypical co-activation of elbow flexion and shoulder abduction (Twitchell, 1951) that becomes visible in reaching (Levin, 1996), in arm-load related reductions in upper limb workspace (Ellis et al., 2009), and in a diminished ability to extend the fingers (Lan et al., 2017; Ellis et al., 2016). Engaging trunk movements in reaching has been considered as movement strategies to compensate for upper limb motor impairments with associations to the level of impairment (Cirstea and Levin, 2000). These stroke-related movement abnormalities 
might become present in isolation or combination and chronically manifested depending on the severity of deficit and cerebral region affected (Raghavan, 2015), thereby presenting a continuous challenge for treatment approaches.

Being able to capture these upper limb movement characteristics is important to improve the understanding of stroke-related movement deficits, including their possible underlying dysfunctions, and to further investigate effectiveness of approaches to influence these deficits (Kwakkel et al., 2017). In this regard, it needs to be considered that upper limb movements can be assessed on different levels. Approaches to evaluate and assess upper limb movement deficits after strokes range from more or less extensive qualitative descriptions of body functions and activities in therapeutic records of clinical practice, over clinical scales that mostly rely on observer-based scoring and time-efficiency measures, to instrumentations and technologies for kinematic motion analysis. Although clinical assessments, such as the Fugl-Meyer Assessment of the Upper Extremity (FMA-UE) or the Action Research Arm Test (ARAT), have demonstrated excellent reliability and validity as assessment tools (Platz et al., 2005), their level of information detail, mostly due to the gross ordinal scoring nature of relatively complex defined movement items, does not allow to sufficiently discriminate physiological and above-mentioned pathological movement behavior (Kwakkel et al., 2017).

Kinematic assessments on the other hand are supposed to offer fine-grained and objective outcomes on movement quality and have shown to detect stroke-related movement impairments in terms of longer movement times, greater trunk displacement and less elbow extension in reaching movements (Collins et al., 2018; Alt Murphy and Häger, 2015). However, the widespread application of kinematic measurements in clinical practice faces several barriers. First, the high variety of measurement systems with different considerations on interaction forces, movement tasks and different metric derivations hampers the comparability and conclusion drawing (Schwarz et al., 2019). Secondly, investigations of complete motion kinematics including trunk and finger motions were sparse. Thirdly, most of the measurement systems being used were optoelectronic and robotic systems that are based on fixed laboratory environments and expensive equipment (Walmsley et al., 2018). Being able to perform comprehensive upper-limb kinematic analysis outside of the laboratory, in flexible environments with the least possible influence on movement behavior would facilitate implementation of kinematic measurements of qualitative aspects movement behavior in clinical practice. In setting up this pilot study, it was aimed to address the outlined limitations by extensively measuring and quantifying reach-to-grasp movements after stroke, with respect to interjoint coordination determined by trunk compensation 
and flexion-extension of the elbow, wrist and fingers, specifically quantified during active grasp and object displacement. A portable inertial system was used to measure complete upper limb kinematics, from the trunk to the fingertip, including fingertip force sensing in flexible experimental tasks and set-up environments. Different task characteristics, such as the target locations and the object to be grasped were considered in the experimental design to investigate influences of additional arm load and workspace relations including increased mechanical work demands in movements against gravity on metrics for determining interjoint coordination. It is assumed that the reaching movement might result in different joint executions with respect to different target locations in the workspace, e.g., features of the pathological flexor pathology might become more pronounced in target positions with higher anti-gravitational mechanical work and with more distance from the body center. Likewise, grasping different object weights results in different additional armloads, that could affect the ability to perform unaffected reaching.

The primary study goal was to evaluate spatiotemporal kinematic metrics for the assessment of upper limb movements after stroke. It was first questioned whether changes in the kinematic range of motion ( $\mathrm{ROM})$ in terms of joint angle ranges can be attributed to the factor tested arm, object weight and target height during object displacement. The second question was how far the kinematic metrics relate to clinically measured upper limb impairment. The third question related to the correlation between each of the joint range metrics to evaluate potential joint coupling, such as the pathological flexor synergy between shoulder flexionextension, elbow flexion-extension and trunk compensation.

\subsection{Methods}

\subsubsection{Study design and participants}

This pilot study was set up to investigate upper limb motion primitives from proximal to distal function in stroke subjects by use of a wearable inertial sensing system. The study was approved by cantonal ethics in Zurich (BASEC-No: Req-2019-00417) and carried out in accordance with the declaration of Helsinki. Subjects after stroke were recruited from a University Hospital Zurich Stroke Registry and invited for a single-session measurement of two hours at the Clinic of Neurology of the University Hospital Zurich, Switzerland.

Subjects were included if they were at least 18 years old, able to give informed consent and had been diagnosed with unilateral stroke at least six months before the study onset with 
associated upper limb impairments. Subjects had to have at least partial ability to move the arm against gravity and to perform finger movements for basic grasp function. Exclusion criteria were pre-existing deficits of the upper limb, such as orthopedic impairments, severely increased muscle tone with limitation in range of motion in the upper limb (Modified Ashworth Scale of $>2$ in one of the upper limb muscle groups), severe sensory deficits in the upper limb (absence of light touch in the hand and fingers), and severe communication or cognitive deficits that cause inability to follow the procedures. Participant characteristics of interest included the gender, age, stroke location side, time since stroke, stroke affected cerebral perfusion territory and the severity of upper limb motor impairment, as measured with FMA-UE. The FMA-UE is a cumulative numerical scoring system to evaluate motor function after stroke, which consists of an arm, wrist, hand and coordination subsection to account for independent severity and recovery patterns, presented in a full score range from 0 to 66 points (Fugl-Meyer et al., 1975).

\subsubsection{Measurement system}

The wearable inertial sensing measurement system was a modified version of the inertial measurement unit (IMU)-based hand and finger sensing system, reported and evaluated by Kortier et al. (2014). It was composed of eight IMUs, with triaxial accelerometers and gyroscopes, based on a micro-controller-based sensing system principles of the PowerGlove (Kortier et al., 2014; van den Noort et al., 2016) that were covered by 3D-printed housings, and combined with force sensors. The IMUs were placed and fixated at the sternum, shoulder, upper arm, lower arm, hand, thumb, index and fingers with medical tape or 3D-printed flexible straps (Figure 6.1). Additionally, the finger IMUs were combined with force-sensitive resistors (FSR) to detect interaction forces between the object to be grasped or manipulated and the finger pad. The upper arm IMU was placed on the lateral side of the arm, close to the elbow and the lower arm IMU was placed on the dorsal side of the forearm, close to the wrist. The hand sensor was placed on the back of the hand, and the thumb, index and middle finger IMUs were attached on the fingertips of the respective fingers, with the forcesensitive resistors fixed on the finger pad by the IMU housing's strap. Each pair of triaxial accelerometers and gyroscopes (ST LSM330DLC manufactured by STMicroelectronics, Geneva, Switzerland) were contained within small printed circuit boards (PCBs). The separately encased IMUs were connected via flexible cabling strips, forming two separate strings (arm string-containing the sternum, shoulder, upper and lower arm sensors; and hand string-containing the hand and fingers sensors). Signals from both sensor strings 
were collected and connected through a bus master/microcontroller (Atmel XMEGA manufactured by Atmel, California, USA) and streamed real-time via the USB channel onto a PC for control in Matlab software (MATLAB version 2016b, The Mathwork, Natick, MA, USA). Acceleration data was collected with a sampling frequency of $100 \mathrm{~Hz}$ and gyroscope data with a frequency of $200 \mathrm{~Hz}$. Both were low-pass filtered by using a Butterworth filter with a cut-off frequency of $10 \mathrm{~Hz}$.

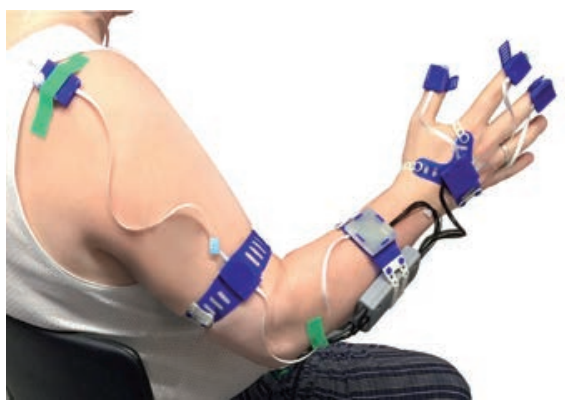

(a)

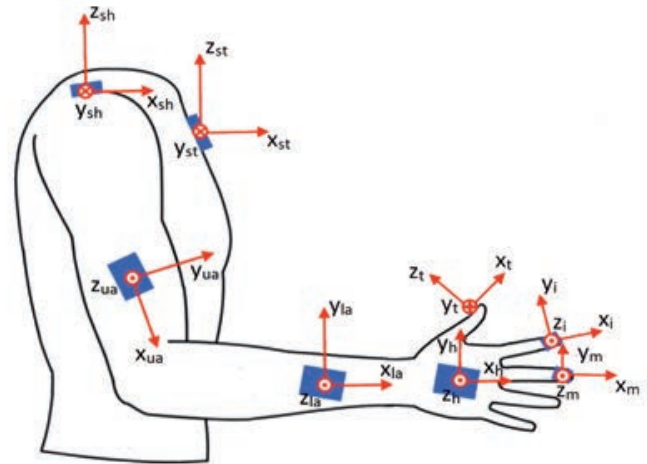

(b)

Figure 6.1. Wearable inertial sensing system: (a) system set-up; (b) anatomical frame definition per segment.

\subsubsection{Kinematic reconstruction}

All sensors were calibrated each day prior to the measurements by placing them inside a box with orthogonal sides, which was turned over 90 degrees in all three orthogonal directions. The accelerometer bias in the different axes and the gyroscope static bias was measured before the whole experiment per subject and compensated during the measurements (Brodie et al., 2008). The kinematic reconstruction was based on the estimation of the sensors' orientation, which is taken from the acceleration and angular velocity measures of the IMUs. In order to estimate the orientation of the limb segments, a sensor-to-segment calibration was performed, as well as a definition of a common global frame for all sensors. The sensor-to-segment calibration was carried out to determine the upper body anatomical axes of the limb segments (joints) relative to the corresponding sensors by performing ten different postures and movements that were based on Luinge et al. (2007) and Ricci et al. (2014). The equipped test person was assisted by a trained research clinician to perform the calibration protocol, which consisted in eight static positions and two dynamic movements, as shown in Table 6.1. 
Table 6.1. Sensor-to-segment calibration protocol

\begin{tabular}{llcc}
\hline & & \multicolumn{2}{c}{ Anatomical axes } \\
\cline { 3 - 4 } No & Calibration position/movement & Left arm & Right arm \\
\hline 1 & Hand held in pronation flat on the metal box & $z_{h^{\prime}}, z_{i}, z_{m}$ & $z_{h^{\prime}}, z_{i^{\prime}}, z_{m}$ \\
2 & Hand held in sagittal plane with $90^{\circ}$ elbow flexion & $-y_{h^{\prime}}-y_{i_{i}},-y_{m}$ & $y_{h^{\prime}}, y_{i^{\prime}}, y_{m}$ \\
3 & Thumb held flat on the metal box & $z_{t}$ & $z_{t}$ \\
4 & Thumb held in sagittal plane with the hand in pronation & $y_{t}$ & $-y_{t}$ \\
5 & Forearm held in pronation along the transversal plane & $z_{l a}$ & $z_{l a}$ \\
6 & Forearm motion from supination to pronation in elbow flexion & $x_{l a}$ & $-x_{l a}$ \\
7 & Upper arm held parallel to the sagittal plane with $90^{\circ}$ elbow flexion & $-x_{u a}$ & $-x_{u a}$ \\
8 & Shoulder horizontal abduction with 90 elbow flexion & $z_{u a}$ & $z_{u a}$ \\
9 & Standing straight & $z_{s h^{\prime}}, z_{s t}$ & $z_{s h^{\prime}}, z_{s t}$ \\
10 & Bending forward by hip flexion until around $60^{\circ}$ & $y_{s h^{\prime}}, y_{s t}$ & $-y_{s h^{\prime}},-y_{s t}$ \\
\hline
\end{tabular}

In the static positions, the gravity vector measured by the accelerometers represents one of the axes. In the dynamic movements, the angular velocity, depending on the rotation direction, also represents the rotation around a specific anatomical axis of a body segment. For each anatomical frame, two different axes were measured using either the accelerometer or gyroscope, depending on the segment. The third axis is calculated using the crossproduct of the previous two axes. Subsequently, an orthonormal coordinate axis was based on these three axes. The last two movements, standing straight and bending by hip flexion, were used to determine the global frame and initial sensor orientation estimation (Kong et al., 2016). The static neutral pose, with the arm stretched along the body and the fingers extended, gives the common vertical axis by measuring the gravity vector in all sensors. The hip flexion movement is performed with the arms extended along the body for the definition of the horizontal axis of the global frame. With the sensor-to-segment alignment and the common global frame for every IMU, it is possible to reconstruct the movement of the trunk, arm, hand, and fingers. Integration drift of the angular velocity over time was corrected by applying a Madgwick filter to correct for the inclination error of the sensor with respect to the gravitational component of the accelerometers (Madgwick et al., 2011). Drifts in the gyroscope orientation were reduced by zero-velocity updates, following the methods of Kirking et al. (2016) where if the norm of the angular velocity is below $3 \% \mathrm{~s}$ is defined to be static in terms of actual sensor movements.

The joint angles are defined as the angle between two anatomical axes of adjacent limb segments of the respective joint as indicated in Figure 6.1b. Positive angles indicate flexion, abduction or supination of a joint and a negative angle indicates extension, adduction or pronation. 
Three trunk compensation angles were calculated by comparing the projected trunk axes onto the global frames corresponding to the static neutral pose, consisting of trunk flexion (rotation in the sagittal plane around the $y$-axis of the sternum), lateral rotation (rotation around the $\mathrm{x}$-axis of the sternum), and torsion (rotation around the $\mathrm{z}$-axis of the sternum). Shoulder flexion/extension was defined as the angular variation of the upper arm's $\mathrm{x}$-axis (x_ua) in the frontal plane (defined by the $\mathrm{x}-\mathrm{z}$ plane of the sternum's frame). Shoulder abduction/adduction is determined by relating the upper arm (x_ua) to the sternum's frame in the frontal plane (defined by the $y$-z plane of the sternum, see Figure 6.1b). Elbow flexion/ extension was determined by the angle between the upper (x_ua) and the lower arm's (x_la) $\mathrm{x}$-axis. Forearm supination/pronation was defined by the mean orientation variation around the $\mathrm{x}$-axis of the lower arm (x_la). Wrist flexion/extension was defined by the angle between the $x$-axis of the lower arm (x_la) and the hand's $x$-axis (x_h). The finger flexion/extension (thumb, index finger and middle finger) was defined as the angle between the $\mathrm{x}$-axis of the hand ( $\mathrm{x} \_\mathrm{h}$ ) and the fingertip frames (x_m, $\mathrm{x} \_\mathrm{i}$ and $\left.\mathrm{x} \_\mathrm{t}\right)$.

\subsubsection{Experimental protocol}

At the beginning, each participant was interviewed about demographic information and assessed for upper limb impairments by use of the FMA-UE (Fugl-Meyer et al., 1975). The experimental protocol was performed on both limbs separately, starting with the non-affected limb (NAF) followed by the affected limb (AF), to study differences between pathological and physiological movement behavior. The protocol consisted in performing reach-to-grasp and displace different types of cubic blocks to different target positions. The participant was positioned sitting in front of a table with the tested arm held in $90^{\circ}$ elbow flexion and the palm facing down on the table. Three markers were defined on the table for placing the hand and fingers in the starting position. The target positions were determined by each participants' maximal arm length in four pre-defined target locations as shown in Figure 6.2 and mirrored between both upper limbs. This task set-up was adapted from the ARAT (Lyle, 1981), which evaluates the ability to grasp and displace, for example wooden blocks, onto a $37 \mathrm{~cm}$ high to-shelf.

The target locations at table height (1, ipsilateral arm length and 2, abducted arm length) and at top-shelf height (3, ipsilateral arm length and 4, abducted arm length) were selected to explore kinematic expressions in a relevant arm workspace and observe effects of arm loading in movements against gravity. The $10 \mathrm{~cm}$ block objects to be grasped varied in three different weights: $108 \mathrm{~g}$ (BL, big light block), $490 \mathrm{~g}$ (BW, big wooden block) and 1008 
$\mathrm{g}(\mathrm{BH}$, big heavy block) to investigate influence of additional load during object grasp and displacement. The weight range of the object was based on the weight of the standardized weight of the wooden block (490 g) that is used in the Action Research Arm Test. The 1 $\mathrm{kg}$ weight was selected as it corresponds to objects, relevant for daily-life functioning daily life, e.g., when manipulating a 11 bottle of water. The lighter block was included to enable the movement analysis with only little additional weight load. The order of blocks was randomized in advance to avoid task-related physical fatigue during the experiments. This resulted in a combination of 12 task conditions per tested arm, that were each repeated three times.

After donning the system, the sensor-to-segment calibration protocol was performed with manual guidance of a therapist to assure proper execution of the static positions and the dynamic movements. Each position was measured for at least five seconds and checked online by an experienced engineer. For accurate global frame definition and sensor orientation estimations, the last two calibration movements were performed before each measurement trial. This procedure allowed to reduce drift in the sensor data during measurements. In between the three repetitions, each subject was asked to avoid extra movements of the tested arm and go back to the starting position as soon as the movement task was finished. This procedure allowed the subjects to rest for about $10 \mathrm{~s}$ between the trials. The whole experiment was expected to be performed within a maximum of $2 \mathrm{~h}$. After the system was donned, each participant rated the wearing comfort of the system, possible limitations of gross movements due to the cables and limitations of grasping due to the fingertip sensors on a 5 -point Likert-scale.
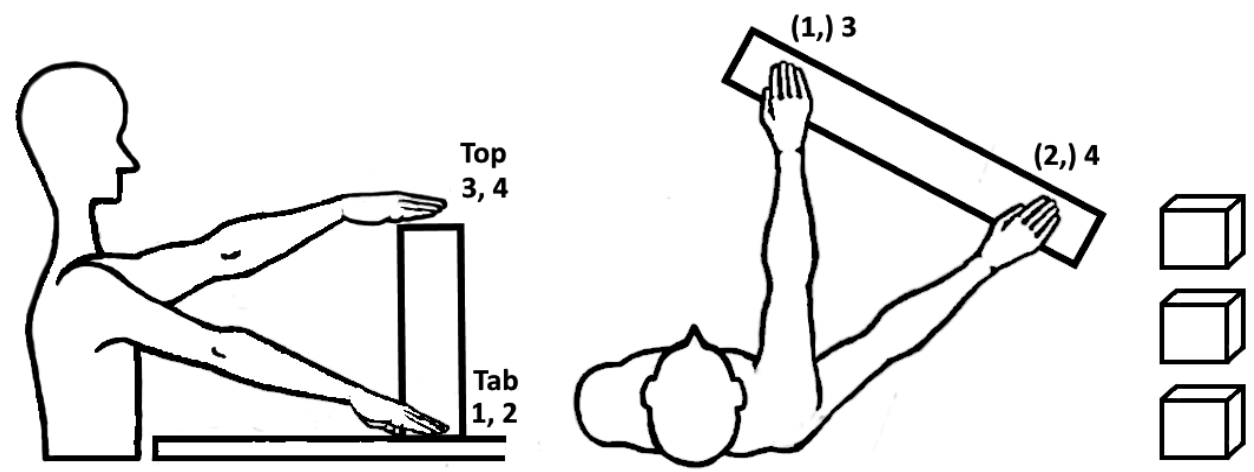

Figure 6.2. Experimental set up in sagittal and top view including the target locations.

Tab 1; in ipsilateral arm length, Tab 2; in abducted arm length, Top 3; ipsilateral arm length, Top 4; in abducted arm length. Block objects: BL (big light block, 108 g), BW (big wooden block, $490 \mathrm{~g}$ ) and BH (big heavy block, $1008 \mathrm{~g})$. 


\subsubsection{Feature extraction}

To enable task-specific spatiotemporal analysis of the reach-to-grasp movements, each movement trial was segmented into three phases: (1) reach, (2) displacement and (3) return by determining the time points of movement onset, grasp, release and movement end. Movement start and movement end were detected using a threshold detection algorithm for the upper arm's IMU angular velocity norm, where a threshold of $0.1 \mathrm{rad} / \mathrm{s}$ was used (de Vries et al., 2018) to account for relevant limb motion. The force data of the fingertips on the table was also used as an onset and offset indicator, where applicable. The moment of grasping was defined by the detection of finger reaction and interaction forces, whereas the release is defined by the decrease in force signal to the lowest value of force, as displayed in Figure 6.3. In cases where no force profile was detected due to low interaction forces or because the finger contact points deviating from the force sensor placements, the grasp and release time points were identified via the joint angle profiles. The release time point was defined by the changes from finger flexion to extension including the maximum elbow extension and shoulder flexion, that represent the moment of maximal reach to target position. The duration of each movement phase was calculated as the time between the delimitating time points of each phase.

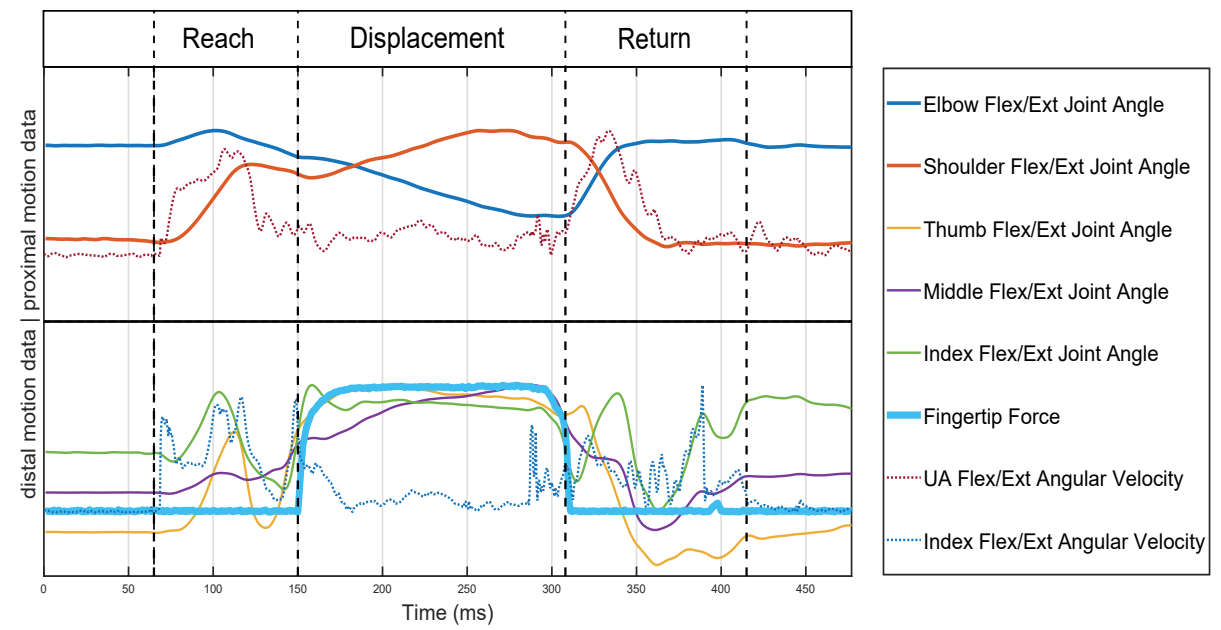

Figure 6.3. Proximal (shoulder, elbow), distal (finger) motion data and force signal for phase segmentation.

The data is scaled to fit the plot, not the actual measured values on the $y$-axis.

For validation of the relevant expected differences between physiological and pathological movement behavior in the study sample, movement time and active range of motion of the main degrees of freedom (DOF) were compared between the affected and non-affected side. 
Movement time was defined as the time between movement start and end, detected by the $0.1 \mathrm{rad} / \mathrm{s}$ threshold. The DOF included trunk compensation, shoulder flexion-extension, shoulder abduction-adduction, elbow flexion-extension, forearm supination-pronation, wrist flexion-extension, thumb, middle finger and index finger flexion-extension for the entire task analysis per target location of the reach-to-grasp movement.

The primary outcome parameters, range of motion in trunk displacement, elbow, wrist, and finger flexion-extension were defined as the difference between the maximum and minimum angle of the joint during the period of object displacement, because they were expected to show expressions of the pathological flexor synergy and compensatory trunk movements. Kinematic parameters of interest to determine interjoint-coordination during the reach-to-grasp movement were defined as the joint ranges in trunk displacement, elbow, wrist, and finger flexion-extension within the displacement phase of the task. Trunk compensation in degrees was used as a metric to quantify the amount of compensatory trunk inclination during the upper limb movement and was defined by the square root of the sum of squares of the ranges in all three trunk compensation angles. Range of motion in elbow and wrist flexion-extension were calculated by taking the difference between the maximum and minimum joint angle measured in the displacement phase, as a metric for quantifying the pathological flexor synergy. The range of motion in finger flexion-extension was calculated as the mean between the range of the index and the middle fingers for each movement execution to consider distal characteristics of the pathological flexor synergy.

\subsubsection{Statistical analysis}

All outcome parameters were visually inspected in histograms and presented descriptively by means and standard deviations.

Differences in range of motion in trunk displacement, elbow, wrist, and finger flexionextension during object displacement were analyzed with respect to tested arm, object weight and target height, by considering the average of the three repetitions per subject and task condition. A linear mixed model analysis was applied to investigate significant differences and interactions between the independent factors, arm (AF, NAF), object (BL, BW, BH) and target height (Tab, Top), on the dependent variable of the metrics on joint range of motion, as presented in the model: Metric - a1 $\times$ Arm $+\mathrm{a} 2 \times$ Weight $+\mathrm{a} 3 \times$ Height $+\mathrm{a} 4 \times$ Subject. The linear mixed model analysis was selected as it takes into account the repeated measures experimental design and inner subject effects in a nested structure of the dependent variables. 
The analysis of the relationship between the displacement phase kinematics of trunk displacement, elbow, wrist, and finger flexion-extension and the individual impairment level, as determined with the clinical FMA-UE test, was explored by plotting the median joint ranges including the upper and lower boundaries of the interquartile range of the affected arm against the measured impairment with the FMA-UE. Statistical testing for answering the second and third research question was performed by cross correlations based on Spearman rank correlations to investigate the relationships between FMA-UE, trunk displacement, elbow flexion-extension, wrist flexion-extension, and finger flexion-extension. All statistical tests were performed using Matlab (MATLAB version 2016b, The Mathwork, Natick, MA, USA) and SPSS (SPSS version 26.0, IBM Corp., Armonk, NY., USA) with a significance level of $\mathrm{p}=0.05$, indicating significances of $\mathrm{p}=0.01$ and $\mathrm{p}=0.001$ specifically.

\subsection{Results}

Kinematic measurements were gathered in 10 chronic stroke subjects within a recruitment period of 8 days in July 2019. One subject performed only two of the three block conditions due to time constraints. The data of the remaining blocks were discarded due to incomplete and incorrect sensor-to-segment calibration data. This resulted in a total of nine out of 10 subjects, who were included in the data analysis, adding up to 324 affected and non-affected side motion data sets. All participants rated the measurement system to be comfortable to wear. One subject rated some influence on the gross movements due to the cable wires of the sensing system. Three of the participants reported impedance of grasp due to the finger sensors.

\subsubsection{Demographics}

The demographics of the study participants are shown in Table 6.2, consisting of four rightside dominant and five left-side affected subjects. Upper limb impairments were measured with the FMA-UE score, ranging from 28 to 46 out of 66 points. Subjects with strokes in the perfusion territory of the middle and posterior cerebral artery showed slight increased upper limb impairments (FMA-UE mean 32.6) when compared to those with strokes in the anterior cerebral artery area (FMA-UE mean 43). According to a group analysis of the upper limb capacity-levels in relation to FMA-UE score (Hoonhorst et al., 2015), this sample included one subject with poor capacity (FMA-UE 23-31), eight subjects showing limited capacity (FMA-UE 32-47) and no subject with notable capacity (FMA-UE 48-52) or full function (FMA-UE 53-66). 


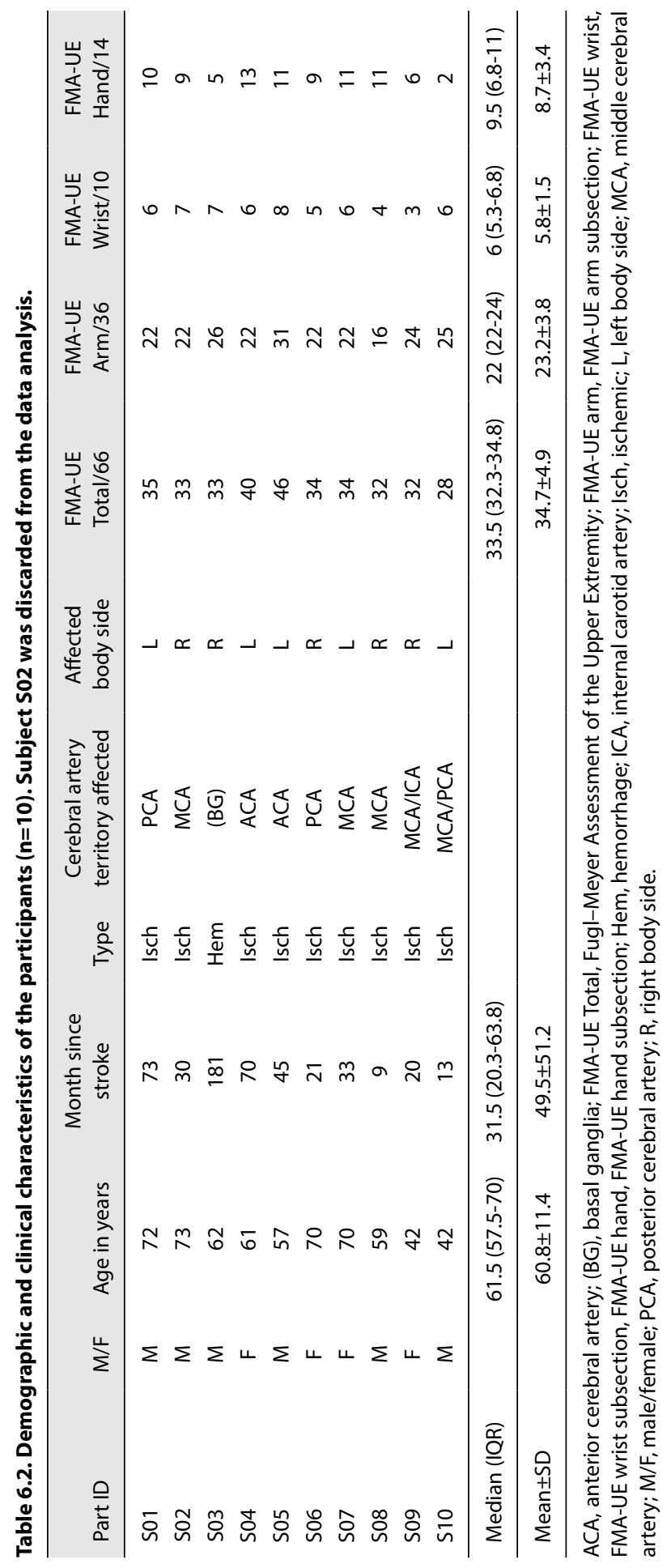




\subsubsection{Upper limb kinematic measures}

The automated detection algorithms were successfully applied in $56.1 \%$ of the data. Corrections had to be made in $47.8 \%$ of the NAF data and $52.2 \%$ of the AF data. Failures in automated detection were $76.5 \%$ related to inconsistent or low force profiles and in $23.5 \%$ related to jerky and noisy angular velocities or joint angle profiles and manually corrected.

Statistically significant higher movement times were found in the AF (mean $4.9 \pm 1.6 \mathrm{~s}$ ) when compared to the NAF (mean $2.8 \pm 5.4 \mathrm{~s}$ ) for the whole task execution $(\mathrm{p}<0.000)$ and accordingly for all subphases $(\mathrm{p}<0.000)$ of the reach-to-grasp movement. The mean difference in ROM across the main DOF between the AF and the NAF was $10.0 \pm 6.9$ degrees across the investigated joints, ranging from 0.2 to 28.7 degrees. The differences in range of motion between the AF and NAF were statistically significant across target locations for shoulder flexion-extension, elbow flexion-extension, wrist supination-pronation, thumb, and index finger flexion-extension, as shown in Supplementary Table S6.1. Range of motion in flexionextension of the shoulder and the elbow were consistently lower in the AF when compared to the NAF, indicating a limited ability to elevate the arm and extend the elbow in reaching. Trunk compensation was significantly different between AF and NAF for the two abducted target locations. A higher mean flexion-extension range was detected for both the index and the middle finger of the AF compared to the NAF, besides lower flexion-extension ranges in the thumb of the affected side for all target positions.

\subsubsection{Influences of the factors, arm, object weight and target height on joint range of motion}

For each of the primary kinematic features (trunk compensation, elbow, wrist, and finger flexion/extension), significant differences in range of motion of the displacement phase can be attributed to the factors tested (arm, object weight, target location). The results of estimates for the independent fixed factors arm (affected side vs. non-affected side), object (BL, BW, BH) and target height (table location vs. top location) on the selected DOF are shown in Table 6.3.

The factor of the tested arm showed significant effects on trunk compensation with larger range of motion in the $\mathrm{AF}$ (mean 9.4 \pm 1.2 degrees) when compared to the NAF (mean 8.2 \pm 1.1 degrees) with $\mathrm{F}=8.327, \mathrm{p}=0.006$. Elbow flexion-extension was significantly lower in the $\mathrm{AF}$ (mean 44.3 \pm 3.9 degrees) than in the NAF (mean 54.2 \pm 4.6 degrees) resulting in significant effects of the arm tested with $\mathrm{F}=23.385, \mathrm{p}=0.000$. Higher ranges in wrist flexion-extension 
were found in the AF (mean 29.4 \pm 4.2 degrees) than in the NAF (21.2 \pm 2.7 degrees) with $\mathrm{F}=30.798, \mathrm{p}=0.000$ and in finger flexion-extensions of the $\mathrm{AF}$ (mean 99.6 \pm 11.4 degrees) when compared to the NAF (mean 77.1 \pm 9.0 degrees) with $F=29.553, p=0.000$.

Significant effects for the fixed factor of object weight were found on the metric of trunk compensation $(\mathrm{F}=4.238, \mathrm{p}=0.022)$. Considering post-hoc pairwise testing, trunk displacement was significantly larger when displacing the big heavy block (mean 10.2 \pm 1.9 degrees) when compared to the displacement of the big light block (mean 7.9 $\pm 1.4, p=0.026$ ) and non-significantly larger in comparison to the big wooden block (mean $8.4 \pm 1.3$ degrees, $\mathrm{p}=0.067)$.

The factor height showed significant effects on all DOF. Displacement to the top height location resulted in significantly higher trunk compensation (mean $9.5 \pm 1.3$ degrees) when compared to table locations (mean $8.1 \pm 1.1$ degrees, $\mathrm{p}=0.006$ ). The highest statistically significant effect was found in elbow flexion-extension with pronouncedly increased range of motion in the top height location (mean 61.3 \pm 4.4 degrees) when compared to the table locations (mean 37.2 \pm 3.9 degrees, $\mathrm{p}=0.000$ ) with $\mathrm{F}=147.742, \mathrm{p}=0.000$. Likewise, ranges in wrist flexion-extension and finger flexion were increased in the top locations with a wrist flexion-extension mean of $23.7 \pm 3.4$ degrees in the table locations when compared to a mean of $26.9 \pm 3.8$ degrees in the top locations $(F=4.354, \mathrm{p}=0.040)$ and a finger flexion-extension mean of $82.1 \pm 9.9$ degrees in the table locations and a mean of $94.4 \pm 11.1$ degrees in the table locations $(\mathrm{F}=7.920, \mathrm{p}=0.006)$.

Table 6.3. Statistical significance of the effects of the independent fixed factors arm, object, and height on the dependent variables of the selected joint range metrics. The factor object including post-hoc pairwise testing between the three levels (BL, BW, BH).

\begin{tabular}{lcccc}
\hline Factor & $\begin{array}{c}\text { Trunk } \\
\text { compensation }\end{array}$ & $\begin{array}{c}\text { Elbow flexion } \\
\text { extension }\end{array}$ & $\begin{array}{c}\text { Wrist flexion } \\
\text { extension }\end{array}$ & $\begin{array}{c}\text { Finger flexion } \\
\text { extension }\end{array}$ \\
\hline Arm (AF vs. NAF) & $0.006^{* *}$ & $0.000^{* * *}$ & $0.000^{* * *}$ & $0.000^{* * *}$ \\
\hline Object (BL, BW, BH) & $0.022^{*}$ & 0.146 & 0.401 & 0.588 \\
-BL vs. BW & 1.000 & 0.680 & 1.000 & 1.000 \\
-BL vs. BH & $0.026^{*}$ & 1.000 & 0.543 & 1.000 \\
-BW vs. BH & 0.067 & 0.156 & 1.000 & 1.000 \\
\hline Height (Tab vs. Top) & $0.006^{* *}$ & $0.000^{* * *}$ & $0.040^{*}$ & $0.006^{* *}$ \\
\hline
\end{tabular}

$*^{* *},{ }^{* * *}$ indicate statistical significance of $\mathrm{p}<0.05, \mathrm{p}<0.01$ and $\mathrm{p}<0.001$, respectively. $\mathrm{AF}$, affected side; $\mathrm{BH}$, heavy block; BL, light block; BW, wooden block; NAF, non-affected side; Tab, table target position; Top, top location. 


\subsubsection{Relationship between kinematic parameters and clinical measures of impairment}

For investigating the relationship between the individual participants' impairment level, as indicated by the FMA-UE score, and the joint ranges of the affected side during displacement, the FMA-UE score was plotted against the subjects median range of motion in trunk compensation and flexion-extension of the elbow, wrist, and fingers as visualized in Figure 6.4a-d. Three repetitions, three block weights and four target positions were considered for each subject resulting in 36 trials per subject and tested arm, represented by median and interquartile range. There was no significant correlations found between the FMA-UE and the individuals mean trunk compensation $(\mathrm{r}=0.11, \mathrm{p}=0.78)$, elbow flexion/extension $(\mathrm{r}=0.00, \mathrm{p}=1.00)$, wrist flexion/extension $(\mathrm{r}=-0.12, \mathrm{p}=0.77)$ and finger flexion/extension $(\mathrm{r}=-0.28, \mathrm{p}=0.46)$.
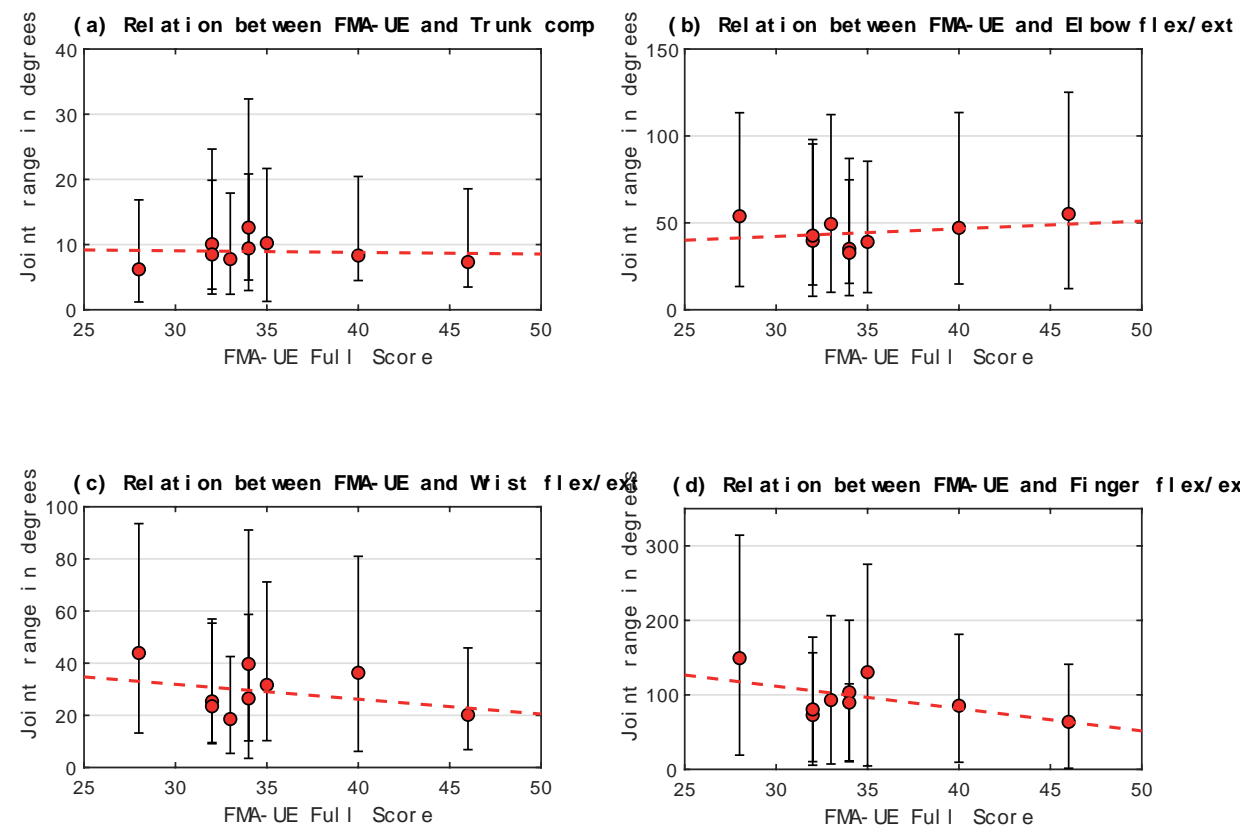

Figure 6.4. Subjects median joint range of (a) trunk compensation, (b) elbow, (c) wrist, and (d) finger flexion/extension of the affected side in relation to impairment level (FMA-UE score ranging from 0-66 points).

Error bars represent the interquartile range over all trials performed by each of the nine subjects and the regression lines over the subjects are included for each metric. 
In a sub analysis, the relationship between the kinematic metric and the related FMA-UE subsection was explored. The correlation between the FMA-UE arm section and trunk compensation resulted in $\mathrm{r}=-0.57(\mathrm{p}=0.11)$. Elbow flexion/extension correlated statistically significantly with the FMA-UE arm subsection with $\mathrm{r}=0.68(\mathrm{p}=0.04)$. The relationship between the FMA-UE wrist subsection and wrist flexion/extension ( $r=0.00, p=0.99)$ as well as between the FMA-UE hand subsection and finger flexion/extension $(r=-0.56, p=0.11)$ was not conclusive.

\subsubsection{Relationship between the selected joint range metrics}

Similarly, the relationship between the selected joint range metrics did not result in significant correlations, except for trunk compensation and elbow flexion/extension. A statistically significant correlation was found between the mean trunk compensation and the elbow flexion/extension in the AF with a negative relationship ( $\mathrm{r}=-0.88, \mathrm{p}=0.0031)$ as shown in Table 6.4. In the NAF statistically significant correlations were found between wrist and finger flexion/extension with strong positive correlations $(\mathrm{r}=0.72, \mathrm{p}=0.0369)$.

Table 6.4. Statistical significance of the effects of the independent fixed factors arm, object, and height

\begin{tabular}{|c|c|c|c|c|c|c|c|c|c|}
\hline $\mathrm{AF}$ & $\begin{array}{l}\text { Trunk } \\
\text { comp }\end{array}$ & $\begin{array}{c}\text { Elbow } \\
\text { flex/ext }\end{array}$ & $\begin{array}{c}\text { Wrist } \\
\text { flex/ext }\end{array}$ & $\begin{array}{l}\text { Finger } \\
\text { flex/ext }\end{array}$ & NAF & $\begin{array}{l}\text { Trunk } \\
\text { comp }\end{array}$ & $\begin{array}{c}\text { Elbow } \\
\text { flex/ext }\end{array}$ & $\begin{array}{c}\text { Wrist } \\
\text { flex/ext }\end{array}$ & $\begin{array}{l}\text { Finger } \\
\text { flex/ext }\end{array}$ \\
\hline $\begin{array}{l}\text { Trunk } \\
\text { comp }\end{array}$ & 1.00 & $-0.88 * *$ & 0.05 & 0.10 & $\begin{array}{l}\text { Trunk } \\
\text { comp }\end{array}$ & 1.00 & -0.35 & 0.08 & 0.17 \\
\hline $\begin{array}{l}\text { Elbow } \\
\text { flex/ext }\end{array}$ & . & 1.00 & -0.32 & -0.20 & $\begin{array}{l}\text { Elbow } \\
\text { flex/ext }\end{array}$ & . & 1.00 & 0.35 & -0.03 \\
\hline $\begin{array}{l}\text { Wrist } \\
\text { flex/ext }\end{array}$ & . & . & 1.00 & 0.53 & $\begin{array}{l}\text { Wrist } \\
\text { flex/ext }\end{array}$ & . & . & 1.00 & $0.72 *$ \\
\hline $\begin{array}{l}\text { Finger } \\
\text { flex/ext }\end{array}$ & . & . & . & 1.00 & $\begin{array}{l}\text { Finger } \\
\text { flex/ext }\end{array}$ & . & . & . & 1.00 \\
\hline
\end{tabular}

* Indicates the statistical significance of the correlation with $\mathrm{p}<0.05$ and ** indicating statistical significance of the correlation with $\mathrm{p}<0.01$.

The relationship between the statistically significant correlations between the DOF, elbow flexion/extension joint ranges against trunk compensation and wrist against finger flexion/ extension joint ranges were further evaluated by visualizing, as presented in Figure 6.5. The linear regression line between the trunk and the elbow joint ranges of the AF was defined by $y=-3.6 x+76$. Linear regression between the wrist and finger flexion/extension joint ranges was described by $y=2.1 x+34$. 

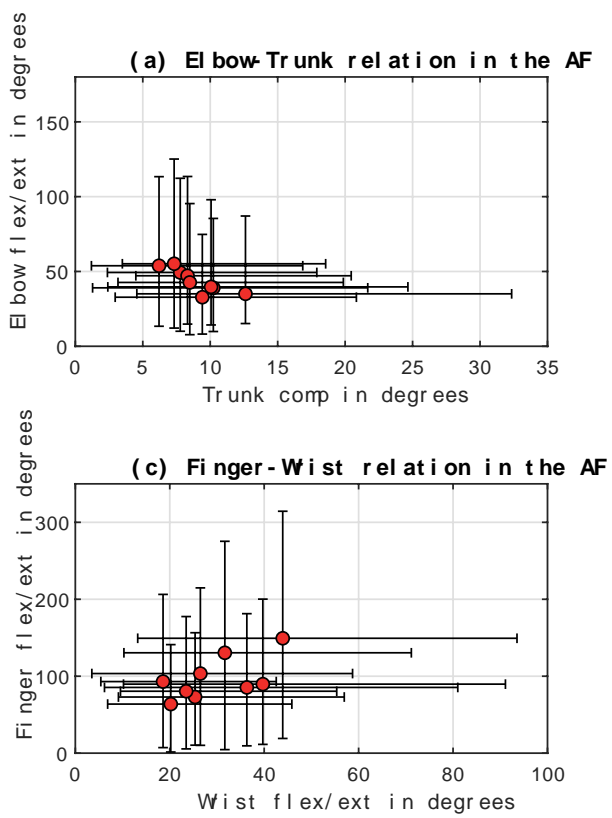
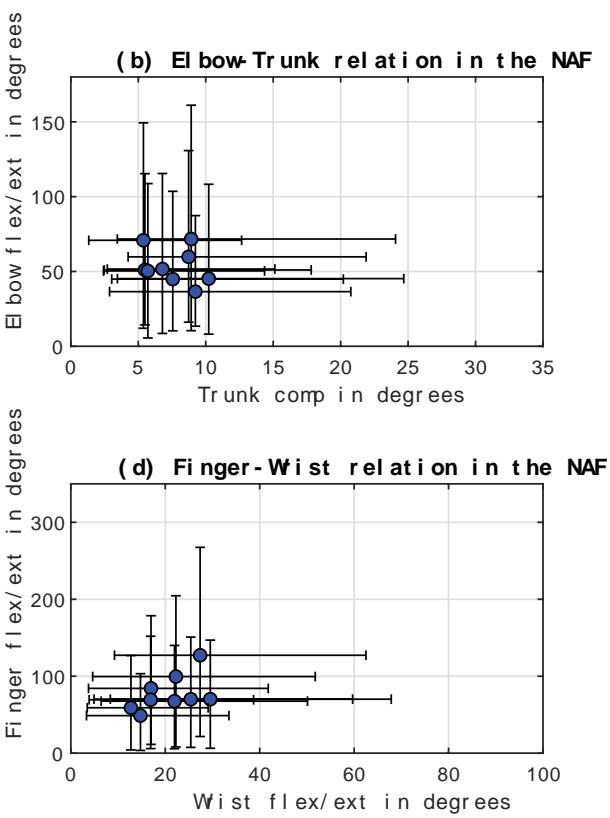

Figure 6.5. Correlations between (a) trunk compensation and elbow flexion/extension in the affected side, (b) trunk compensation and elbow flexion/extension in the non-affected side, (c) wrist and finger flexion/ extension of the affected side, and (d) wrist and finger flexion/extension of the non-affected side.

\subsection{Discussion}

In this pilot study, sensor-based upper limb kinematic measurements of reach-to-grasp and displacement activities executed by chronic stroke subjects were used to examine and relate characteristics of movement impairments and to explore the influences of additional weight loads and mechanical work requirements on the upper limb kinematics. Movement impairments, such as longer movement times and decreased range of motion across the upper limb DOF were found in the affected when compared to the non-affected side, supported by existing literature (Alt Murphy and Häger, 2015; van Kordelaar et al., 2012). Besides the evidence for weakness and impaired interjoint coordination in the affected upper limb, illustrated in the consistently decreased shoulder flexion and elbow extension for the whole task execution, this study focused on investigating the expression of pathological coupling between the trunk, elbow, wrist and fingers during object displacement within maximal arm length, as most clearly represented in Table 6.4. In order to include distally pronounced aspects of movement behavior in the kinematic analysis of object grasping and displacement, the range of motion of the wrist and the fingers' flexion-extension has been included in the analysis. The increased finger flexion in the AF when compared to the NAF expands on the 
characterization of the pathological flexor synergy and confirms previous research by Miller and Dewald (2012) and Lan et al. (2017) that described and increased difficulty to release the finger flexion with increased arm load. The significant positive correlation between finger and wrist flexion/extension in the non-affected upper limb, as shown in Table 6.4, could be interpreted as a physiological movement synergy allowing the subject to perform efficient grasp function. Herein, factors impacting the force and mechanical work demands were examined to prove the load-dependent appearance of pathological joint coupling in the upper extremity.

On the level of trunk compensatory movements, increased trunk movements were found in tasks with the affected arm when displacing the heavy block, that could be related to a compensation of weakness in the proximal shoulder muscles or weakness of the trunk muscles themselves. If trunk weakness itself was present in the investigated population, this could account as one explanation for why trunk compensatory movements were also detected in the non-affected side of the data set. It can be assumed that trunk weakness itself would diminish the ability to counterbalance an additional arm weight with either the affected or the nonaffected limb. Another explanation for increased trunk movements in the non-affected side could be based on the fact that the NAF arm might deviate from complete healthy movement behavior due to indirect deficits in the non-crossing pathways from the ipsilesional cortex (Nowak et al., 2008). Nevertheless, these findings are in line with Repnik et al. (2018), who investigated the parameters movement time, smoothness, hand trajectory similarity and trunk stability in stroke subjects and healthy subjects when performing the ARAT and found similarly differences in trunk movements, especially early at movement onset, besides also noting occasional trunk motions above $10^{\circ}$ in healthy subjects (Repnik et al., 2018). These findings suggest that the trunk compensation feature should be further studied with respect to diagnostic sensitivity and specificity to quantify stroke-related upper limb impairments. Apart from trunk compensation, an increase of the object weight showed no significant effects on the features of flexion-extension range of motion of the elbow, wrist, and fingers.

Differences related to the target height factor were detected in all tested features and can be partially explained by the different movement trajectory and positioning of the block object with respect to the hand posture between the top shelf and the table locations. The differences in wrist and finger flexion/extension can in part be explained by differences in hand positioning with respect to the target location, e.g., the hand might be more flexed in the wrist when displacing the block to the top shelf. Nevertheless, the strongest effect of target height was found in the elbow flexion/extension ROM, with a mean increase of elbow 
range of motion $24.0 \pm 5.9$ degrees in the top shelf locations when compared to the table target locations. This study finding was surprising, since all four target locations were defined by the maximum arm length to assure the requirement of complete elbow extension at the end of the displacement phase. Furthermore, the increased elbow flexion/extension motion in movements with increased gravity impact stand in contrast to previous research and the hierarchical structure of the synergistic movement patterns ((Twitchell, 1951; Fugl-Meyer et al., 1975), that presume an increased difficulty of uncoupling elbow flexion from shoulder flexion with increased load and motion. The present study's findings, contrarily, could suggest that range of motion in elbow extension is increased in target positions that have a larger distance to the subjects' body center and require increased mechanical work against gravity. Despite the tentativeness of these results and the small study population, these outcomes could open new intervention strategies and should be addressed in future research with larger study samples to investigate possible underlying mechanisms. If the identification of factors that influence the increase or decrease in pathological joint coupling is possible, new intervention approaches would be opened to sustain stroke-related movement impairments. Including gradual decrease or increase of the armload has shown benefits for determining the severity of pathological joint coordination and providing patient-centered interventions, as indicated by Ellis and colleagues (Ellis et al., 2016). The examination of the influence of task conditions on the selected DOFs support the definition of the task-dependent and dynamic appearance of the pathological flexor synergy (Sukal-Moulton et al., 2007; Lan et al., 2017; Ellis et al., 2016). In the current study, the body of research on task-dependent changes based on planar movement task evaluations were extended to evaluations of reach-to-grasp activities in non-laboratory environments with a close linkage to functional activities of daily life. The fact, that we did not find significant effects of the object weight on the upper limb features, elbow, wrist, and finger flexion-extension except for trunk compensation, might be due to the range of object weight selected, from $100 \mathrm{~g}$ to $1 \mathrm{~kg}$. Considering previous research on arm loading during reaching reported a maximum additional load of $50 \%$ of the arm weight (Ellis et al., 2016) that would result in about $2 \mathrm{~kg}$ for an average person of $80 \mathrm{~kg}$ and an arm weight of around 5\% of the body weight. Nevertheless, the subjects included in this study showed considerable difficulty in grasping and displacing the $1 \mathrm{~kg}$ heavy block.

These findings on movement condition effects on the relevant kinematic features stress the importance of considering task-dependent influences, such as gravitational forces and biomechanical constraints, when assessing and treating stroke-related upper limb impairments. Cortes et al. (2017) studied arm motor control in a planar robotic device and 
found a non-linear relationship between two-dimensional pointing parameters and scores from clinical scales incorporating antigravity strength demands. The authors suggested that arm motor control plateaus at 5 weeks post-stroke, whereas strength improvement, as measured by clinical scales, continues to improve up to 54 weeks post-stroke (Cortes et al., 2017). In this regard, it would be interesting to extend these objectives to three-dimensional movement tasks and investigate whether arm motor control, when measured in more complex reach-to-grasp movements by use of less motion impeding measurement systems, follows a similar recovery scheme when compared to 2D arm motor control and clinical scales. The usage of wearable sensing allows movement quality to be tracked in terms of kinematics in a less obstructive and more flexible way.

Another question addressed in this study was the relationship between the kinematic features of the displacement phase and the clinically measured individual impairment level. No clear correlations were found between the kinematic metrics and the FMA-UE, whereas trunk compensation and elbow flexion/extension showed strong correlation with the FMA-UE arm subsection, as well as the correlation between the FMA-UE hand subsection and finger flexion/extension. These findings are in line with existing research (Schwarz et al., 2019; Kanzler et al., 2020) and support the fact that kinematic parameters are, rather, complementary than redundant to standard clinical scales and potentially add clinically relevant information. The large interquartile ranges in all measured DOF in all study subjects illustrates the large variability in movement execution especially in non-cyclical discrete motions. The negative correlation between trunk compensation and elbow flexion/extension in the movements of the affected limb can be interpreted as an expression of the pathological joint coupling in stroke, where trunk compensation is increased relatively to the lack of active range of motion in the elbow during reaching. The significant positive correlation between the wrist and finger flexion/extension in the non-affected side could account for the appearance of physiological movement synergies during grasping and displacement that is less strong including larger interquartile ranges in both joints and non-significant in the affected side. These results support the use of the selected spatiotemporal features by use of non-laboratory kinematic movement analysis to assess aspects upper limb movement quality and impairments after stroke. Capturing and analyzing the relevant joint ranges during functional activities provides additional complementary information concerning how functional movements are performed and thereby help to overcome limitations of most existing clinical scales. Being able to detect the main aspects of movement quality and impairments allows selecting and monitoring changes in functional outcome and planning 
interventions that target these aspects. Future research should consider and re-evaluate the outcome features and task considerations presented herein on larger sample sizes to further underpin existing evidence of sufficient validity and reliability for metrics of joint range of motion and trunk displacement (Schwarz et al., 2019; Kanzler et al., 2020). Furthermore, analysis of the assessments' clinimetric properties should be extended to domains sensitivity and specificity for differentiation physiological and pathological movement behavior.

\subsubsection{Implementation of the device and analysis methods in clinical practice}

Wearable devices for assessments of motor function have been an ongoing research direction over the last decade. Portable devices facilitate the setup time and do not require patients to be directed to specific labs for measurements. The presented system potentiates the objective monitoring of the patients' impairments and provides the therapists an additional and more precise information about the movements' profile. The collective use of visual observations by the clinician and objectively measured patient movements using a sensing system as proposed in the current study system is intended to be used as means to provide better diagnostic and, thus, better therapy outcomes by providing a more thorough evaluation. Further research should focus on a clinician's point of view in the usability of the system in the clinic. By instructing therapists on how to use and analyze the distributed measuring system and its output, it is possible to obtain feedback, both from the patient and therapist, on its usability and relevance. In future, and after iterating the development steps of the device and methodology based on the feedback received, objective measurements with these types of system can become the standard for motor function evaluation.

\subsubsection{Strength and limitations}

As a main limitation of this pilot study, the small sample size of the study needs to be considered as a factor that suppresses the robustness and degree of reliance of the findings and results presented. However, the investigated sample was homogenic with respect to a limited upper limb capacity, as determined by Hoonhorst et al. (2015), and allowed exploration of the applicability of the multisensory wearable system in the target population at an early device development stage (Hoonhorst et al., 2015). The study sample included was intended to be able to perform reach-to-grasp and displace movements, which excludes more severely affected subjects. Nevertheless, besides the similar overall upper limb impairments, the included subjects showed reasonable variation in terms of the deficit distribution in the corresponding limb segments, as depicted in Table 6.2. 
The principal idea of combining multiple sensing modalities, such as inertial sensing and other signal quantities, in a wearable system for upper limb kinematic motion analysis was considered as a strength of the device used, as this allows both for simplification or extension of the measurement modalities and enables the conduction of neurophysiological and biomechanical experiments on post-stroke upper limb movements in relatively unrestricted measurement surroundings. The wearable measurement system presented here combined complete kinematic motion analysis of the main DOFs of the upper limb kinematic chain and interaction force measurements at the fingertip, that have shown to be a powerful tool in reach-to-grasp detection and could further inform through measurements of grasp control. Although, we could confirm the application for assessing upper limb movements in chronic stroke subjects in this pilot study, the usability in clinical practice, including set-up, running and analyzing and the selected outcomes, would need to be addressed in future research.

Unfortunately, the force-sensitive resistor sensors used in this study showed limitations in capturing low forces per area and diminished flexibility to adapt to the shape of the finger pad and the grasped object. Therefore, grasp force could not be quantified as an outcome measure apart from the phase segmentation detection. An advanced version of flexible fingerprint sensors, as described in Wolterink et al. (2018) is intended to be incorporated in the next generation of this multisensory measurement device. Detecting normal and shear force during grasp can provide further insights into movement control and effectiveness (Nowak et al., 2005). The combination of kinetic and kinematic measurements would allow to further study grasp control and stroke-related deficits, such as force limitation due to weakness or findings on force overshoot (Parry et al., 2019). Effective grasping is undertaken by placing single fingers perpendicularly to the object surface (Cuijpers et al., 2004). This could be further explored in subjects after stroke with more adequate kinetic measurements.

Another considerable limitation relates to the systems' measurement accuracy. Similar to other IMU sensors, the systems' measurement accuracy depends on a successful sensor and sensor-to-segment calibration, appropriate filtering and fusion algorithms and reliable segment and joint angle definitions (Walmsley et al., 2018). The accuracy of measurements was assured by updates of the global frame orientation definition and the avoidance of unnecessary extra movements prior to each task execution, which lasted not longer than nine seconds.

The detection of phases related to the movement primitives of reaching, object transport and return was feasible by a set of automatic detection algorithms in $47.8 \%$ of the affected 
upper limb data and $52.2 \%$ of the non-affected movement data. The observer-based validation of the points for phase discrimination and manual correction of defective time points to differentiate movement phases remain limited to subjective decision-making and time-consuming in processing. The grasping and release point, defined by an increase and decrease of the force profile and/or angular velocity in flexion-extension of the index finger, could show deviations due to inconsistent finger motion and force signals. In particular, the point of object release was difficult to detect when no distal signal peaks were detectable and could be affected by a systematic error if, for example, only maximum elbow extension is used to determine object release, which has to be considered rather as an indirect assumption than a proof of object release. Additionally, periods of transition or "dead time" between the phases need to be considered, as for example at movement start and end, where indifferent minor motions could affect the threshold detection. The application of improved flexible fingertip force sensors would reasonably improve the accuracy and reliability of time-points for phase detection of reach, displacement and return that are in alignment with studies on comparable movement analysis (Parry et al., 2019; Repnik et al., 2018). The accurate and time-efficient detection of motion primitive phases of reach-to-grasp activities is a relevant requirement for comparable and repeatable motion analysis of upper limb function.

Finally, we acknowledge that beside movement time and joint range of motion, several other kinematic parameters could have been investigated, such as hand trajectories or smoothness measures to complement the picture of movement quality and impairments. Based on the fact that signal information for the parameter calculation is provided by the system, this could be addressed in future studies using this multisensory measurement device. The data acquired in this study was publically made available for transparent reporting and re-evaluation and extension of the results (Schwarz et al., 2020). To realize the long-term goal of upper limb kinematic assessments in clinical practice, this pilot study investigated metrics that were appropriate to detect and quantify impaired movement behavior after stroke by use of a wearable inertial measurement system. Even though the suggested metrics were derived from well-defined movement tasks, it is reasonable to include these metrics in existing analysis, that have been proven to be useful in the evaluation of non-structured daily-life activities (van Meulen et al., 2016; Held et al., 2018). Additionally, considering movement task characteristics and factors influencing the movement behavior were key to enable the evaluation of subject-specific motion aspects and assessing the dynamics of the impairments. 


\subsection{Conclusion}

This pilot study demonstrates the applicability of sensor-based kinematic motion analysis of functional reach-to-grasp and displacement movements in chronic stroke subjects with limited upper limb capacity by use of a wearable inertial sensing system. Relevant features to determine upper limb upper limb movement quality were suggested and examined for influences caused by the tested arm, object weight, target height factors and with respect to clinically measured impairment level. Range of motion in trunk displacement, elbow, wrist, and finger flexion-extension showed considerable differences between the AF and the NAF. Effects on metrics for interjoint coordination, as defined by the features, trunk compensation, elbow, wrist, and finger flexion-extension during displacement were found for the factors of an increase in object weight and target height. Hence, the factor's object weight and target height were suggested to study expressions of the pathological flexor synergy in functional reach-to-grasp movements with different task conditions. The significant correlations between elbow flexion/extension and trunk compensation detected in the affected upper limb support the appearance of pathological joint coupling during object displacement. Range of motion in elbow flexion-extension tended to be lower in the affected side when compared to the non-affected. The finger flexion-extension ROM showed significant differences between the AF and NAF and between the target heights, supporting further evaluation of this feature to quantify distally pronounced aspects of the pathological flexor synergy. These findings support the assessment of kinematic features of reach-to-grasp and displacement movements by use of IMUs and, therefore, help in paving the path towards clinically meaningful and feasible upper limb kinematic assessments in stroke research and clinical practice. The additional investigations on the effect of additional arm load and target height revealed relevant findings in the field of neurophysiology with respect to pathological joint coupling after stroke and highlight important considerations for upper limb kinematic assessments and possible treatment strategies to restore quality of movement in order to regain functionality in activities of daily life. 


\subsection{References}

Alt Murphy M, Häger CK. Kinematic analysis of the upper extremity after stroke-How far have we reached and what have we grasped? Phys Ther Rev. 2015;20:137-55. doi: 10.1179/1743288x15y. 0000000002

Bernstein NA. The Coordination and Regulation of Movements; Pergamon Press Ltd.: London, UK; 1967.

Brodie MA, Walmsley A, Page W, Brodie MA. The static accuracy and calibration of inertial measurement units for 3D orientation. Comput Methods Biomech Biomed Eng. 2008;11:641-8. doi: $10.1080 / 10255840802326736$

Byblow WD, Stinear CM, Barber PA, Petoe MA, Ackerley SJ. Proportional recovery after stroke depends on corticomotor integrity. Ann. Neurol. 2015;78:848-59. doi: 10.1002/ana.24472

Cirstea CM, Levin MF. Compensatory strategies for reaching in stroke. Brain. 2000;123:940-53. doi: 10.1093/brain/123.5.940

Collins KC, Kennedy NC, Clark A, Pomeroy VM. Getting a kinematic handle on reach-to-grasp: A meta-analysis. Physiotherapy. 2018;104:153-66. doi: 10.1016/j.physio.2017.10.002

Cortes JC, Goldsmith J, Harran MD, et al. A Short and Distinct Time Window for Recovery of Arm Motor Control Early After Stroke Revealed With a Global Measure of Trajectory Kinematics. Neurorehabil Neural Repair. 2017;31:552-60. doi: 10.1177/1545968317697034

Cuijpers RH, Smeets JB, Brenner E. On the Relation Between Object Shape and Grasping Kinematics. J Neurophysiol. 2004;91:2598-606. doi: 10.1152/jn.00644.2003

de Vries J, van Ommeren A, Prange-Lasonder G, Rietman J, Veltink PH. Detection of the intention to grasp during reach movements. J Rehabil Assist Technol Eng. 2018;5:1-9. doi: 10.1177/ 2055668317752850

Ekstrand E, Rylander L, Lexell J, Brogårdh C. Perceived ability to perform daily hand activities after stroke and associated factors: A cross-sectional study. BMC Neurol. 2016;16:208. doi: 10.1186/ s12883-016-0733-x

Ellis MD, Carmona C, Drogos JM, Traxel S, Dewald JP. Progressive abduction loading therapy targeting flexion synergy to regain reaching function in chronic stroke: Preliminary results from an RCT. In Proceedings of the 2016 38th Annual International Conference of the IEEE Engineering in Medicine and Biology Society (EMBC), Orlando, FL, USA, 6-20 August 2016 (pp. 5837-40).

Ellis MD, Lan Y, Yao J, Dewald JP. Robotic quantification of upper extremity loss of independent joint control or flexion synergy in individuals with hemiparetic stroke: A review of paradigms addressing the effects of shoulder abduction loading. J Neuroeng Rehabil. 2016;13:95. doi: 10.1186/s12984-016-0203-0

Ellis MD, Sukal-Moulton T, Dewald JPA. Progressive shoulder abduction loading is a crucial element of arm rehabilitation in chronic stroke. Neurorehabil Neural Repair. 2009;23:862-9. doi: $10.1177 / 1545968309332927$

Feix T, Romero J, Schmiedmayer HB, Dollar AM, Kragic D. The GRASP Taxonomy of Human Grasp Types. IEEE Trans Hum Mach Syst. 2016;46:66-77. doi: 10.1109/thms.2015.2470657

Fugl-Meyer AR, Jääskö L, Leyman I, Olsson S, Steglind S. The post-stroke hemiplegic patient. 1. a method for evaluation of physical performance. Scand J Rehabil Med. 1975;7:13-31. 
Held JPO, Klaassen B, Eenhoorn A, van Beijnum BJF, Buurke JH, Veltink P, Luft AR. Inertial Sensor Measurements of Upper-Limb Kinematics in Stroke Patients in Clinic and Home Environment. Front Bioeng Biotechnol. 2018;6:27. doi: 10.3389/fbioe.2018.00027

Hoonhorst MH, Nijland RH, van der Berg JS, Emmelot CH, Kollen BJ, Kwakkel G. How Do FuglMeyer Arm Motor Scores Relate to Dexterity According to the Action Research Arm Test at 6 Months Poststroke? Arch Phys Med Rehabil. 2015;96:1845-9. doi: 10.1016/j.apmr.2015.06.009

Jeannerod M. The representing brain: Neural correlates of motor intention and imagery. Behav Brain Sci. 1994;17:187-202. doi: 10.1017/s0140525x00034026

Jones LA, Lederman SJ. Human Hand Function. Oxford University Press: New York, NY, USA; 2006. Kanzler CM, Rinderknecht MD, Schwarz A, et al. A data-driven framework for selecting and validating digital health metrics: Use-case in neurological sensorimotor impairments. NPJ Digit Med. 2020;3:80. doi: 10.1038/s41746-020-0286-7

Kirking B, El-Gohary M, Kwon Y. The feasibility of shoulder motion tracking during activities of daily living using inertial measurement units. Gait Posture. 2016;49:47-53. doi: 10.1016/j.gaitpost. 2016.06.008

Kong W, Sessa S, Zecca M, Takanishi A. Anatomical Calibration through Post-Processing of Standard Motion Tests Data. Sensors. 2016;16:2011. doi: 10.3390/s16122011

Kortier HG, Sluiter VI, Roetenberg D, Veltink PH. Assessment of hand kinematics using inertial and magnetic sensors. J Neuroeng Rehabil 2014;11:70. doi: 10.1186/1743-0003-11-70

Kwakkel G, Lannin NA, Borschmann K, et al. Standardized measurement of sensorimotor recovery in stroke trials: Consensus-based core recommendations from the Stroke Recovery and Rehabilitation Roundtable. Int J Stroke. 2017;12:451-61. doi: 10.1177/1747493017711813

Lan Y, Yao J, Dewald JP. The Impact of Shoulder Abduction Loading on Volitional Hand Opening and Grasping in Chronic Hemiparetic Stroke. Neurorehabil Neural Repair. 2017;31:521-9. doi: $10.1177 / 1545968317697033$

Levin MF. Interjoint coordination during pointing movements is disrupted in spastic hemiparesis. Brain. 1996;119:281-93. doi: 10.1093/brain/119.1.281

Luinge H, Veltink PH, Baten CTM. Ambulatory measurement of arm orientation. J Biomech. 2007;40: 78-85. doi: 10.1016/j.jbiomech.2005.11.011

Lyle RC. A performance test for assessment of upper limb function in physical rehabilitation treatment and research. Int J Rehabil Res. 1981;4:483-92. doi: 10.1097/00004356-198112000-00001

Madgwick SOH, Harrison AJL, Vaidyanathan R. Estimation of IMU and MARG orientation using a gradient descent algorithm. In Proceedings of the 2011 IEEE International Conference on Rehabilitation Robotics, Zurich, Switzerland, 29 June-1 July 2011 (pp. 1-7).

Miller JC, Dewald JPA. Involuntary paretic wrist/finger flexion forces and EMG increase with shoulder abduction load in individuals with chronic stroke. Clin Neurophysiol. 2012;123:1216-25. doi: 10.1016/j.clinph.2012.01.009

Nowak DA, Grefkes C, Dafotakis M, Eickhoff S, Küst J, Karbe H, Fink GR. Effects of Low-Frequency Repetitive Transcranial Magnetic Stimulation of the Contralesional Primary Motor Cortex on Movement Kinematics and Neural Activity in Subcortical Stroke. Arch Neurol. 2008;65:741-7. doi: 10.1001/archneur.65.6.741

Nowak DA, Rosenkranz K, Topka H, Rothwell J. Disturbances of grip force behaviour in focal hand dystonia: Evidence for a generalised impairment of sensory-motor integration? J Neurol Neurosurg Psychiatry. 2005;76:953-9. doi: 10.1136/jnnp.2004.043943 
Parry R, Soria SM, Pradat-Diehl P, Marchand-Pauvert V, Jarrassé N, Roby-Brami A. Effects of Hand Configuration on the Grasping, Holding, and Placement of an Instrumented Object in Patients With Hemiparesis. Front Neurol. 2019;10:240. doi: 10.3389/fneur.2019.00240

Platz T, Pinkowski C, van Wijck F, Kim IH, di Bella P, Johnson G. Reliability and validity of arm function assessment with standardized guidelines for the Fugl-Meyer Test, Action Research Arm Test and Box and Block Test: A multicentre study. Clin Rehabil. 2005;19:404-11. doi: 10.1191/ $0269215505 \mathrm{cr} 832 \mathrm{oa}$

Raghavan P. Upper Limb Motor Impairment After Stroke. Phys Med Rehabil Clin N Am. 2015;26: 599-610. doi: 10.1016/j.pmr.2015.06.008

Repnik E, Puh U, Goljar N, Munih M, Mihelj M. Using Inertial Measurement Units and Electromyography to Quantify Movement during Action Research Arm Test Execution. Sensors. 2018;18:2767. doi: 10.3390/s18092767

Ricci L, Formica D, Sparaci L, Lasorsa FR, Taffoni F, Tamilia E, Guglielmelli E. A New Calibration Methodology for Thorax and Upper Limbs Motion Capture in Children Using Magneto and Inertial Sensors. Sensors. 2014;14:1057-72. doi: 10.3390/s140101057

Sacco RL, Kasner SE, Broderick JP, et al. An Updated Definition of Stroke for the 21st Century: A statement for healthcare professionals from the American Heart Association/ American Stroke Association. Stroke. 2013;44:2064-89. doi: 10.1161/str.0b013e318296aeca

Santello M, Lang CE. Are Movement Disorders and Sensorimotor Injuries Pathologic Synergies? When Normal Multi-Joint Movement Synergies Become Pathologic. Front Hum Neurosci. 2015;8:1050. doi: 10.3389/fnhum.2014.01050

Schwarz A, Bhagubai MMC, Wolterink G, Held JPO, Luft AR, Veltink PH. Kinematics of reach-tograsp and displacement after stroke [Data set]. Zenodo. http://doi.org/10.5281/zenodo.3930752, Available online: https://zenodo.org/record/3930752\#.X0EGBjVCRhE (accessed on 22 August 2020).

Schwarz A, Kanzler CM, Lambercy O, Luft AR, Veerbeek JM. Systematic Review on Kinematic Assessments of Upper Limb Movements After Stroke. Stroke. 2019;50:718-27. doi: 10.1161/ strokeaha.118.023531

Stinear CM, Barber PA, Smale PR, Coxon JP, Fleming MK, Byblow WD. Functional potential in chronic stroke patients depends on corticospinal tract integrity. Brain. 2007;130:170-80. doi: 10.1093/brain/awl333

Sukal-Moulton T, Ellis MD, Dewald JP. Shoulder abduction-induced reductions in reaching work area following hemiparetic stroke: Neuroscientific implications. Exp Brain Res. 2007;183:215-23. doi: 10.1007/s00221-007-1029-6

Twitchell TE. The Restoration of Motor Function Following Hemiplegia in Man. Brain. 1951;74:44380. doi: 10.1093/brain/74.4.443

van den Noort JC, Kortier HG, van Beek N, Veeger DHEJ, Veltink PH. Measuring 3D Hand and Finger Kinematics-A Comparison between Inertial Sensing and an Opto-Electronic Marker System. PLoS ONE. 2016;11:e0164889. doi: 10.1371/journal.pone.0164889

van Kordelaar J, van Wegen EEH, Kwakkel G. Unraveling the interaction between pathological upper limb synergies and compensatory trunk movements during reach-to-grasp after stroke: A crosssectional study. Exp Brain Res. 2012;221:251-62. doi: 10.1007/s00221-012-3169-6 
van Meulen FB, Klaassen B, Held J, Reenalda J, Buurke JH, van Beijnum BF, Luft A, Veltink P. Objective Evaluation of the Quality of Movement in Daily Life after Stroke. Front Bioeng Biotechnol. 2016;3:210. doi: 10.3389/fbioe.2015.00210

Vos T, Allen C, Arora M, et al. Global, regional, and national incidence, prevalence, and years lived with disability for 310 diseases and injuries, 1990-2015: A systematic analysis for the Global Burden of Disease Study 2015. Lancet. 2016;388:1545-602. doi: 10.1016/S0140-6736(16)31678-6

Walmsley CP, Williams SA, Grisbrook TL, Elliott C, Imms C, Campbell A. Measurement of Upper Limb Range of Motion Using Wearable Sensors: A Systematic Review. Sports Med Open. 2018;4:53. doi: 10.1186/s40798-018-0167-7

Wolterink GJW, Sanders RGP, Krijnen G. Thin, Flexible, Capacitive Force Sensors Based on Anisotropy in 3D-Printed Structures. In Proceedings of the 2018 IEEE Sensors Applications Symposium (SAS), New Delhi, India, 28-31 October 2018 (pp. 1-4). doi: 10.1109/icsens.2018.8589584 


\subsection{Supplementary material}

As a proof of concept validation, differences between the affected and non-affected sides in movement time and range of motion of the main DOFs were tested for statistically significant differences between the dependent groups by use of the Wilcoxon signed rank test, as illustrated in Supplementary Figure S6.1 and Supplementary Table S6.1. For movement time, all trial of the affected and non-affected sides were considered, whereas range of motion was compared between affected and non-affected sides, evaluation all trials separately for each target location.
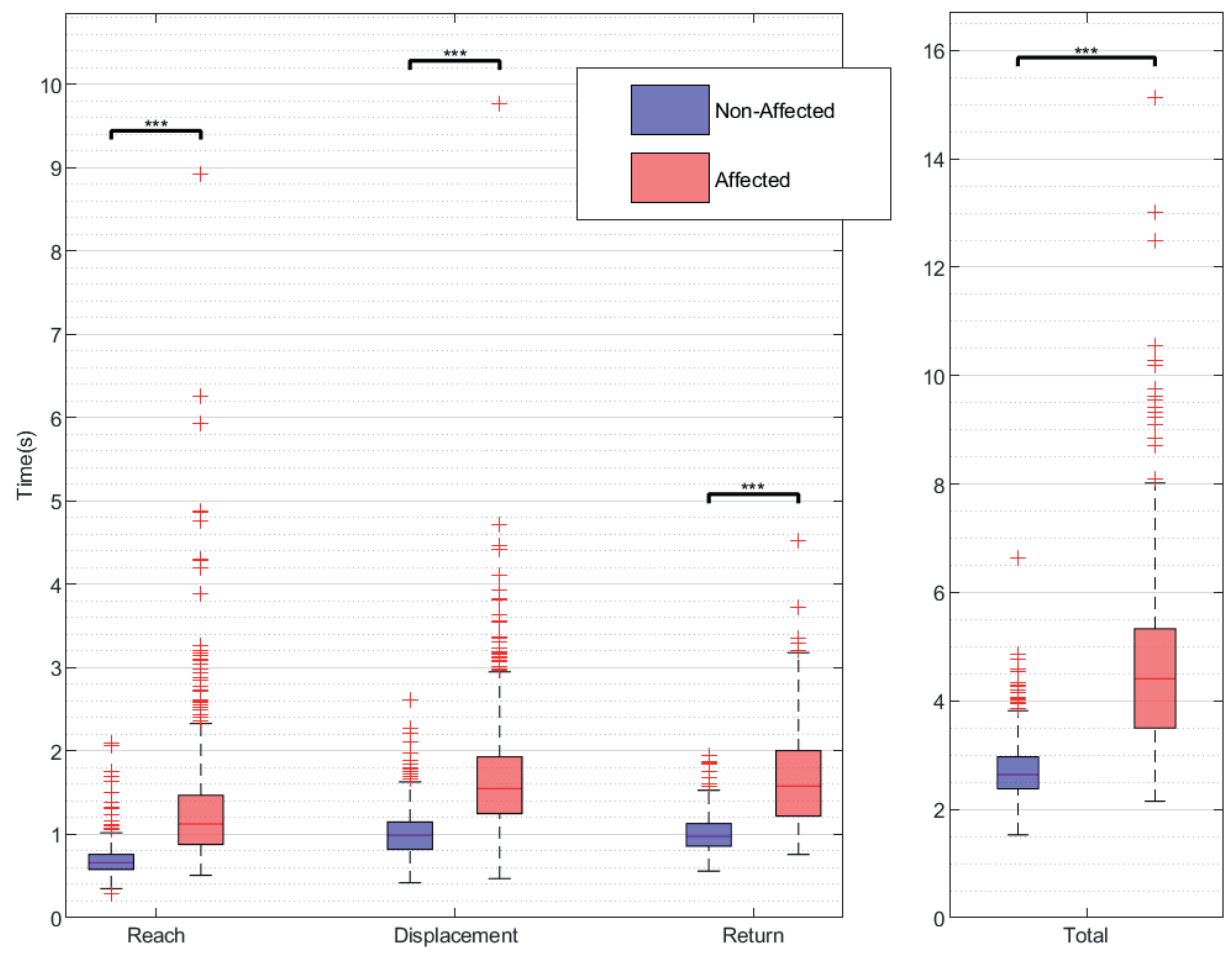

Supplementary Figure S6.1. Movement time in seconds represented for the phases of reach, displacement and return and the total movement time.

Data include all trials, object weights and target positions for the affected side (AF) and non-affected side (NAF). 


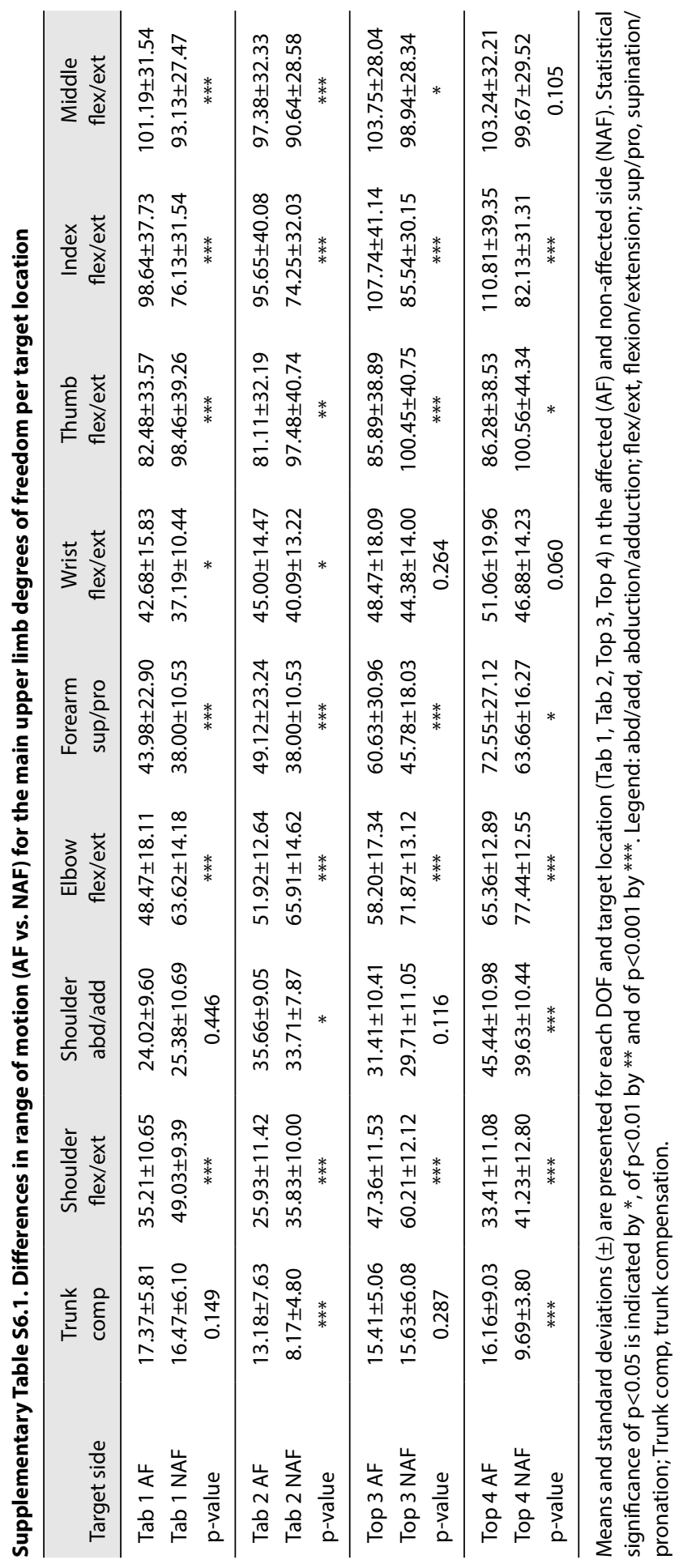





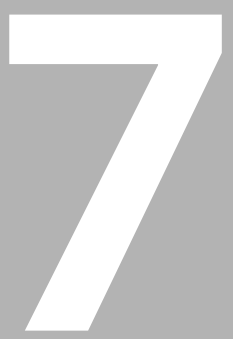

General discussion 

The main goal of this thesis was to investigate opportunities for assessing upper limb movement quality after stroke by use of kinematic sensing technologies. Three studies laid the groundwork for the analysis presented herein to answer the thesis research questions: I. What is the state of the art in kinematic assessments after stroke? II. How can upper limb interjoint coordination be determined? III. What is the effect of gesture versus grasping movement tasks across different stroke-related upper limb impairment severities? IV. Is it possible to detect similar motion primitives of reach to grasp and reach to gesture distally and proximally across different tasks? V. What is the effect of armload and target height on upper limb kinematics in stroke subjects?

With the first study, an overview on available kinematic assessment protocols including the investigated outcome metrics has been provided (chapter 2). The questions, which assessment tasks were applied, by which kind of measurement system, and on which impairment level of stroke subjects, were essential to handle the expected heterogeneity in approaches and capture the whole picture of the state of the art. Regarding the outcome measure qualities of kinematic assessments, the investigated metrics and their clinimetric properties were of major interest.

It has been found that only a limited number of the identified studies investigated the validity, reliability, and responsiveness of the kinematic parameters (31 studies out of 225 included studies). Based on the best-available evidence identified in this systematic review, recommendations were provided on the assessment task, target population, the measurement system, and outcome parameters.

The results and recommendations of the systematic review were considered in planning, running, and analyzing the second study on assessing upper limb pathological synergies after stroke by use of a wearable kinematic sensing system, presented in the chapters 3 to 5. Kinematic data from chronic stroke subjects of at least six months post-stroke, with mild to moderate upper limb motor impairments was recorded during different movements, like single-joint movements, gesture movements, and reach-to-grasp and manipulate objects, to capture the full content of upper limb functionality in terms of workspace, task complexity, and the degrees of freedoms actively involved. Besides kinematic measures to quantify interjoint coordination in the shoulder-elbow-trunk complex (chapter 3), movement variability metrics based on functional PCA (chapter 4), and a kinematic coreset including efficiency, spatial, speed and smoothness measures (chapter 5), were investigated on a set of upper limb movements. The impact of the upper limb movement task condition on 
movement kinematics has been confirmed, as well as the effect of the impairment severity on kinematics throughout the chapters.

In the third study, the kinematic measures were expanded to the first three finger digits and fingertip force sensing by use of a distributed sensing system to investigate movement impairments during reach-to-grasp and displacement tasks (chapter 6). Besides the extension to the finger kinematics as an importent factor of prehensility, the effect of different object weights and target locations on the kinematic measures was explored. The study results suggested that kinematics such as flexion/extension of the shoulder, elbow, wrist, and fingers and trunk compensation were influenced by the target height and object weight. Furthermore, a significant relationship between trunk compensation and elbow flexion/extension during object displacement has been found.

Both studies support the usability of inertial sensing systems, consisting of five or eight IMUs with the fingers included, to perform qualitative analysis of spatiotemporal movement characteristics. These results underpin the strengths of wearable sensing to enable precise and objective assessments of movement quality in flexible measurement settings, which likely leads to future implementation in clinical routine.

The main topics, "the state of the art in upper limb kinematic assessments", "findings on upper limb interjoint coordination", "applicability of wearable sensing for qualitative motion analysis", and "assessment task considerations" will be discussed in the following sections.

\subsection{The state of the art in upper limb kinematic assessments}

The systematic review, presented in chapter 2, revealed 225 includable primary studies on upper limb kinematic assessments after stroke and thereby reflects the most comprehensive and systematic overview til date. It has been shown that kinematic assessments were performed in stroke subjects with moderate to mild impairments (FMA-UE median 41.60, IQR 26.5-48.6) and predominantly in the chronic stage (172 studies compared to 47 studies in the acute and subacute stage). The majority of movement tasks were allocatable to $2 \mathrm{D}$ pointing tasks $(n=81), 3 D$ pointing tasks $(n=67), 3 D$ reach-to-grasp tasks $(n=50)$, and $2 D$ shape drawing task $(n=16)$. An association between the movement task difficulty in terms of gravity influences between $2 \mathrm{D}$ and 3D movements, the task complexity, and the severity of upper limb impairment, resulted in the suggestion to choose $3 \mathrm{D}$ assessment tasks including grasping activities in subjects scoring $\geq 30$ points in the FMA-UE. 
The large number of different kinematic parameters $(n=151)$ and inconsistent reporting on metric derivations and interpretations with respect to the clinical picture of strokerelated upper limb movement disorders highlighted the need for future investigations and improvements in assessment and reporting standards. Only $41 \%$ of the identified metrics were investigated for at least one clinimetric property in 30 of the 225 included studies. The lack of studies on reliability, measurement error and responsiveness of kinematic parameters was discussed in chapter 2 and partially explained by the fact that such methodological research requires careful study planning and reasonably large numbers of subjects and resources besides the difficulty to receive funding for such important but less innovative research topics. The Figures $2.2 \mathrm{~A}$ and B, $2.3 \mathrm{~A}$ and B, and 3 summarize the frequency of metric usage and type and quality of psychometric property evaluation per movement task group and thereby provide information about lacks in knowledge and future research targets.

Based on the best available evidence, frequency of usage and the insight from motor control, we recommended kinematic parameters for trunk/shoulder/elbow and wrist/hand/finger movements for improving comparability in future research. The number of movement onsets and ends, task movement time, path length ratio, the number of velocity peaks, shoulder flexion/extension angle, trunk displacement, and peak velocity were further recommended as a kinematic metric coreset that should be considered when assessing upper limb movements after stroke. The kinematic parameters were included in the observational study presented in chapter 3 to 5 and the pilot study presented in chapter 6.

Besides the findings of this review, an expert opinion of the second Stroke Recovery and Rehabilitation Roundtable "metrics" task force has been followed, suggesting measurement protocols including 2D planar reaching, and 3D functional tasks, such as the drinking task that will allow future pooling of data across sites (Kwakkel et al., 2019). The suggested kinematic coreset in standardized protocols will further help to characterize and understand different impairment mechanisms. Upper limb impairments might be expressed in altered upper limb dexterity, reaction time, movement patterns, and weakness that are detectable by decreased mean velocity, increased movement time, trunk displacement and decreased smoothness of the hand path. Additionally, as illustrated in the figures of chapter 2 on the metric distributions per movement quality construct, it remains challenging to select the right set of outcome measures for reflecting the relevant aspects of movement quality after stroke. The movement quality domains of smoothness and interjoint coordination are overlapping in terms of the definition and selected outcome parameters, as for example the no of movement units (Rohrer et al., 2002; Subramanian et al., 2013). In extension to 
the presented findings, metrics for assessing smoothness and interjoint coordination after stroke are further investigated.

\subsection{Findings on upper limb interjoint coordination}

One of the main questions addressed in this thesis was how to quantify upper limb interjoint coordination after stroke? Interjoint coordination is associated with the neuroscientific concept of synergies that can be seen as principal control patterns of the human brain to cope with the high dimensionality in terms of degrees of freedom (Bernstein, 1967). The concept of motor synergies has been defined as co-activation patterns of motor units in the time and /or frequency domain (Santello et al., 2015) and tackled in different fields of research, ranging from experiments in basic science over clinical examinations to recently emphasized machine-learning approaches for robotic control (Averta et al., 2020). In delineation between physiological and pathological synergistic coupling, typical upper limb pathological synergies after stroke were described as coactivation of shoulder flexion and abduction with elbow flexion that result in limited reaching ability and inability to adapt to specific movement task demand (Levin et al., 2016). Hence, the loss of coordinated muscle or movement patterns after stroke is reflected in a more fixed or constrained set of coordinated movement patterns, or the smaller number of synergies associated with pathological synergistical coupling (Cheung et al., 2012; Klochkov et al. 2018) that lead to inefficient spatial and temporal arrangement of the DOF towards the movement goal (Tomita et al., 2017).

The selection of sensitive and specific measures for quantifying upper limb interjoint coordination comes along with several difficulties, considering the above-mentioned understanding of movement synergies and the state of the art in stroke research and clinical care, as addressed in this thesis. The represented definitions do not include factors of upper limb movements, such as, what are the forces that need to be controlled, nor the number of degrees included, or task complexity in terms of multi-joint coordination. After stroke, selective motor control might be disrupted on different body segments, between the whole upper body and upper limb or between the first three digits in specific upper limb positions. Even though clinical examinations of hierarchically ordered synergistic movement patterns, such as by use of the Brunnstrom stages or the FMA-UE, constitute the gold standard assessment for evaluating post-stroke voluntary movement control, they provide only limited qualitative information on movement behaviour and the severity of the pathological joint coupling (Krakauer and Carmichael, 2017). The FMA-UE was chosen 
as the main outcome comparator for assessing interjoint coordination in the presented studies. This choice was made, because the FMA-UE represents the best-available and widespread measure of independent joint control in subjects after stroke and thereby allows investigating the construct validity of the kinematic parameters for assessing interjoint coordination. As addressed in this thesis, kinematic measures of the main degrees of freedom available through technology-based motion analysis were performed to address the above-mentioned influenting factors of force, the number of degrees of freedom involved and the task-complexity to quantify upper limb interjoint coordination. The question of appropriate outcome measures of interjoint coordination based on chapter 2 has been addressed in chapter 3. Additionally, the question on associations between the selected metric expressions and the movement task content has been investigated. In chapter $\mathbf{4}$, the assumption on diminished movement variability in the affected upper limb has been confirmed in a subsample of moderately impaired subjects for the performance of a set of daily life activities. Finally, the question of the effect of arm load and target height on upper limb kinematics during functional reach to grasp is addressed in chapter 6.

As described in chapter 3 on interjoint coordination after stroke, the relationship between the shoulder and elbow has shown to be different in isolated FMA-UE based joint movements and more functional movements, such as pointing ahead, reach-to-grasp a cup, and insert a key into a lock. Measures of shoulder-elbow-trunk interjoint coordination have shown to be highly task dependent. The results suggest that the movements evaluated in the FMA-UE do not correspond well with functional movements of daily life. Shoulder elbow interjoint coordination, when examined in terms of the angle-angle plot curve shape, has shown to be comparable between the affected and non-affected side in functional tasks, additionally supported by the non-significant results between the tested arms for the metric shoulder-elbow correlation coefficient. In conclusion, characteristics of interjoint coordination should be further investigated in daily life activities to uncover relevant impeding aspects of the dysfunction.

Chapter 4 concerned the quantification of movement complexity during a set of daily living tasks, when estimated by the application of fPCA and associated measures. The principal idea of this approach was that stroke subjects with the presence of pathological synergies show a decreased interjoint coordination and consequentially less movement variability and adaptability during the execution of a complex and comprehensive task set of daily life activities that should be reflected in dimension-reduction-approach measures, such as functional principal components (fPCs). It has been shown in a subsample of four chronic stroke subjects, that the fPCs and reconstruction errors in the affected side and more severe 
affected subjects were lower than in the unaffected side, suggesting less adaptability to the task requirements reflected by lower movement variability. Thereby, it could be confirmed that movements in the affected limb are associated with more constraint and fixed movements in the seven main DOFs that provides promising information on decreased movement adaptability and the severity of these coordination deficits.

Different analytical approaches including dimensionality reduction methods on human motor control measures or cluster-based analysis (Scano et al., 2017) are applicable in the sense of the synergy-concept and common recruitment structures across different movement goals. Upper limb interjoint coordination can be examined between two joints, based on angle-angle plots (as investigated in chapter 3 ) or multiple joints (as presented in chapter 4), requirering different mathematical approaches to represent or reduce the multidimensionality of the motion data, as shown in this thesis.

Besides these findings, some aspects in the terminology of pathological synergies or interjoint coordination after stroke could have been concluded. Strong associations between interjoint coordination and weakness or diminished movement activation were found, represented in the diminished ranges in the target joints. Correlations between spasticity, weakness, and contractures were previously described (Ada et al., 2008). The fact that the target joint positions were not achieved in the affected limb could be a product of diminished muscle force or increased joint stiffness due to co-activation or hypertonia alike and it is questionable whether both coordination and force can be fully dissociated. The Newton's law of motion indicates the strong associations between motion and internal and external forces, such as muscle force and the constant impact of gravity force (Lu et al., 2012). These findings are in accordance with the research of Dewald and colleagues who demonstrated significant effects of armload on pathological synergy expression and provided robotic-based assessment and treatment approaches (Ellis et al., 2016; Ellis et al., 2018). Implementing staged force, speed, and coordination difficulty levels in assessments of upper limb interjoint coordination can be recommended to further explore the relations between both movement aspects. The feasibility and informativeness of such assessment protocols were demonstrated in chapter 6, where effects of different object load on upper body joint kinematics were explored. Associations between trunk compensation and object weight were detected, as well as between trunk compensation and elbow flexion/extension during object transport and displacement. Determining factors that ease or exaggerate pathological joint coupling are important to improve our understanding in the pathomechanisms and to create effective treatment strategies. The fact, that a viscous resistance training to address weakness and 
the presence of the flexor synergy, has not supported group differences in favour of the resistance training group in a RCT (Ellis et al., 2018), points out the challenge to apply specific treatments to improve either weakness or interjoint coordination or both.

The presented studies in this thesis focused on interjoint coordination observed on the level of kinematic signal processing. As visualized in Figure 7.1, interjoint coordination or synergybased control is processed on different levels and has been investigated by means of surface EMG, kinematic analysis, TMS, or EEG. We have not addressed or measured co-activation on the muscular level. It could be that subliminal muscle activation, not sufficient to elicit observable movements, is overseen by limiting the investigation to kinematic measures. Nevertheless, kinematic measures were thought to be appropriate to capture the phenotype or output of stroke-related movement disorders. Measures of central nervous control of the movement synergies can further be included in future research, as depicted in Figure 7.1 , to investigate central nervous signals associated with motor control. Interhemispheric competition, the integrity of the corticospinal tract and recently excessive activation in the reticulospinal tract, were associated with upper limb motor impairments and the presence of the flexor synergy (Yao et al., 2009; McPherson et al., 2018).

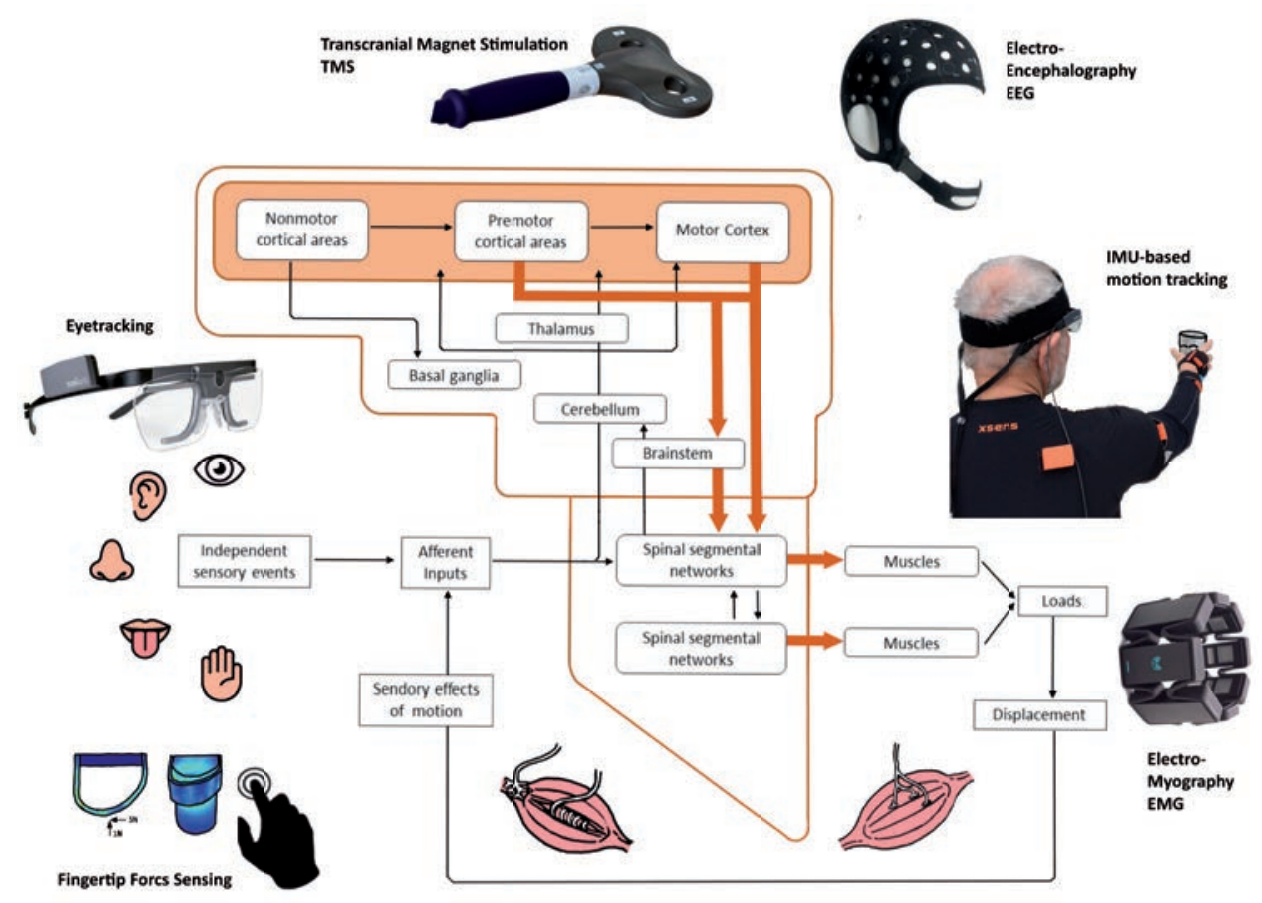

Figure 7.1. Sensorimotor network and applicability of different measurement signals. 


\subsection{Upper limb kinematic measures in wearable applications}

Besides scepticism against the accuracy IMU-based wearable devices, kinematic measures have been acknowledged for their non-obtrusiveness, objective, and continuous measurements enabling ADL recordings to characterize the subjects' motor function, thereby potentially enabling the provision of personalized therapies (Maceira-Elvira et al., 2019). Recommendation to rely solely on so-called assessment "gold standards" in qualitative motion analysis, such as optoelectronic measurement systems (Kwakkel et al., 2019), can be questioned in light of cost effectiveness, usability, given the fact that both methods, since not measuring the exact invitro joint rotation, are subject to imprecision (Lu and Chang, 2012).

The accuracy of sensor measurements in degrees has been reported in eight studies for trunk motions and upper limb motions with measurement errors ranging from $0.7^{\circ}$ to $8.3^{\circ}$ (CuestaVargas et al., 2010) and about $20^{\circ}$ in the thoracic and lumbar spine (Wong et al., 2008). It has been confirmed that measurement errors are influenced by the appropriate selection of fusion and filter algorithms. One of the main concerns in using IMU-measurements for motion tracking of stroke subjects is the reliability of the implemented fusion-algorithms, since stroke subjects tend to move slower and in more segmented motion fragments than healthy subjects (Levin et al., 2016). Measurement errors due to orientation drift are a result of the integration of angular velocity and were suggested to be corrected by sensor fusion and the definition of a resting pose that can be frequently repeated during measurements (Wittmann et al., 2019). In the presented studies in chapter 3 to 5, the start and end positions of the movement tasks were predefined and the recordings were online observed via the avatar and joint angle inspection in the recording software. In chapter $\mathbf{6}$, the accuracy of measurements was secured by recalibration to a predefined resting pose prior to each movement execution. Both wearable systems used in chapters 3 to 6 included IMUs for each of the main upper limb segments to enable the detection of joint kinematics. The Xsens MVN Awinda system consists of high end IMUs that acquire data at $1 \mathrm{kHz}$ (Paulich et al., 2017), demonstrating reasonable accuracy and repeatability of measurements with ICC for all joints ranging between 0.90 and 0.94 and measurement errors between $1.5^{\circ}$ and $2.1^{\circ}$ for single pose calibrations in standing and sitting (Robert-Lachaine et al., 2017). Since the segment-to-sensor calibration defines relevant aspects of rules and regularities of sensor fusion, its performance accuracy is crucial for appropriate measurement accuracy. In the second and third study presented in chapter 3 to 6 , manual assistance was provided to the subjects to hold the required pose, especially the fully extended arm during sensor-to- 
segment calibration to ensure a proper calibration quality. The fact that further development and refinement of algorithms to fuse and filter motion signals is ongoing, likely improves the performance of sensing technology and robustness of results in future applications.

Besides the measurement accuracy of wearable kinematic sensing, the validity of measures is of interest. As pointed out in chapter 2, validity was most frequently investigated with respect to standard clinical scales, such as the FMA-UE, showing moderate to strong correlations. In chapter 3, we demonstrated comparable results for validity of shoulderelbow joint kinematics, captured with a full-body wearable sensor suit when correlated with the FMA-UE in accordance with previous research (van Meulen et al., 2015). However, as discussed in chapter 2 , correlations between continuous kinematic measures and ordinal clinical scales need to be interpreted with caution. Kinematic measures provide continuous less constraint outcomes on the level of movement quality and impairment when compared to low-level ordinal scales and therefor potentially provide more precise and discriminating information. However, the expression of kinematics largely depends on the movement context that was measured and therefor needs to be accounted for when analyzing and interpreting the validity of kinematics. For example, trunk motion kinematics captured during a reach-to-grasp task could result in stronger correlations with the FMA-UE or the ARAT when compared to trunk kinematics that were captured during a planar pointing task. Depending on the kinematic movement context and the selected clinical scale, a strong correlation between these measures could be associated with redundancy, while a lower correlation could be interpreted with some additional or different value of either of them. Another aspect that is included in the precise measurement characteristics of upper limb kinematics and constitutes an obstacle in the analysis, is the within and between subject movement variability and the lack of normative data. A recent systematic review and meta-analysis on the responsiveness of kinematic and clinical measures of upper limb motor function after stroke found contrastingly to previous research a significantly higher responsiveness in the Motor Activity Log questionnaires for amount and quality of use, when compared to the path length ratio, trunk and shoulder joint ranges, time to peak velocity, and peak velocity (Villepinte et al., 2020). Villepinte and colleagues followingly highlighted the differences between subjective questionnaires and more objective kinematic outcome measures and pointed to possible influences on their findings due to differences in reaching with and without grasp in the kinematic assessment protocols. These results underline the necessity to further monitor the relations between clinical and kinematic measures in subjects after stroke. Hypothesis on the relationship between kinematic metrics and clinical scales 
should be phrased from the beginning by considering the scale dimension and the number of movement items, that the measure is based on. A data-driven framework for selecting and validating digital health metrics includes several validation steps, such as sensitivity, specificity, reliability, and validity tests for improving the quality of kinematic metrics as an upper limb outcome measure (Kanzler et al., 2020).

Another aspect related to kinematic measures in wearable applications is the usability und usefulness in clinical practice. The measurement devices, used in the studies presented in chapter 3 to 6, consisted of several IMUs (five to eight) attached to the main upper body segments of the subject. This setup, even though coming with some obtrusiveness, was the best balance between measurement quality and detail and feasibility of capturing complex upper limb movement data in different measurement surroundings. Instrumented wearable motion analysis has shown to be a powerful tool to capture and describe movement quality on a continuous basis and thereby supporting health care professionals in monitoring the progress and decision-making processes. Given the available evidence that measures such as movement duration, path length ratio or smoothness measures provide the best-available information of upper limb movement quality after stroke (Alt-Murphy and Häger, 2015; Schwarz et al., 2019; Li et al., 2015), support the usage of single-IMU measurements, since all these metrics can be derived from a wrist or hand worn IMU. Using a single IMU for assessing such aspects of upper limb movement quality would likely increase the clinical implementation and the patients and therapists acceptance of usage. The fact that wearables are appraised for their potential increase of health care availability and sustainability of the interventions into areas of the patients' daily life which, likely increases the frequency of usage resulting in longterm reduced costs related to productivity loss and informal care (Maceira-Elvira et al., 2019).

\subsection{Assessment task consideration}

Kinematic outcomes provide information about movement quality on the level of body functions, as indicated in Figure 1.4. ICF Framework, while being capturable in different scenarios ranging from strength or range of motion testing on the body functional level to reach-to-grasp movements on the activity and participation level. The purpose of the upper limb movement in terms of the movement task has been addressed specifically throughout the chapters of this thesis. The importance of considering the movement purpose can be justified by the large variability of upper limb movements. Different to lower limb function that is 
mainly assessable in cyclic and repetitive tasks, such as gait or cycling, most of upper limb functions are based on discrete movements with varying demands on distally coordinated actions (Hogan et al., 2007). The upper limb might be used to stabilize oneself, to grasp, hold or manipulate different objects or simply in terms of gesture movements to underline expressions in communication. Differences between reach-to-point and reach-to-grasp movements in healthy as well as stroke subjects were described previously (Jeannerod 1990; McCrea, 2002; Mesquita, 2019).

As identified in chapter 2 , the movement tasks selected for upper limb kinematic assessments after stroke, ranged from 2D planar pointing or shape-drawing tasks to 3D pointing, 3D reach to grasp, and other tasks. The other tasks were mainly based on isolated movement elevations or measurement device specific tasks, such as gamifications and hardly generalizable apart from their primary investigation. It can be assumed that the assessment movement task is influenced by the possibilities or barriers related to the device, as well as by the capability of the assessed person to perform the movement. It has been shown that $2 \mathrm{D}$ movement tasks were more applicable to subjects with lower upper limb function (FMA-UE $<30$ points) while $3 \mathrm{D}$ movement tasks were likely to be performed by subjects with better upper limb function (FMA-UE $\geq 30$ points), especially if grasp-actions are included. These impairmentbased differences alongside with the complexity of upper limb functionality supports the development of a standardized task set protocol of upper limb movements that covers the full range of motor performance ability, as recommended by the SRRR “metrics" task force (Kwakkel et al., 2019). The authors suggested performance assays to assess isolate core motor execution capacities outside of a task context, such as 2D reaching, finger individuation, grip strength and precision grip strength, and a functional task assay of the drinking task in subjects an FMA-UE $\geq 32$ points. Such a task set facilitates across-study comparability and sensitive longitudinal tracking of recovery in subjects.

Assessing natural movements, such as the drinking task (Alt Murphy, 2018) with higher ecological validity than a planar robotic-based tracking task for example, has found the basis for the experiments in chapter 3 to 5 . A set of 30 daily living activities, shared within the European SoftPro project collaboration, formed a representative dataset of upper limb daily living activities to quantify movement complexity in chapter $\mathbf{4}$ and confirmed task-related differences between gesture movements without object contact and grasp movements with object contact and manipulation in chapter 5 . The gestures and bimanual activities included in this set, have been rarely investigated in existing studies, although relevant in daily life. It has been shown that a considerable number of reach-to-grasp activities contain coupled 
movements of both hands, when one stabilizes while the other manipulates (Rose et al., 2004). The included stroke subjects in the herein presented research had moderate to mild upper limb impairments with FMA-UE scores ranging from 32 to 61 and were all tested in the chronic stage ( $\geq 6$ months after the acute stroke event). Consequentially, the results could be different in more severely impaired subjects. It remains further to be determined, in how far subjects with cognitive or communicative impairments are able to follow kinematic assessment protocols. Including movement tasks with a close relation to real-world activities in assessment protocols, such as presented in chapter 3 to 5 , could improve the applicability in such populations.

Based on the presented findings in this thesis and the phrased expert recommendations, a reduced task-set could consist of a 2D planar task, a 3D discrete task, a 3D functional task, a bimanual task, and dual task condition, as illustrated in Figure 7.2. Since a lot of upper limb activities of daily life require parallel processing of several information, the assessment of dual task conditions might provide important insides in quantifying and understanding differences in capacity and real-life performance. Only one study analyzed influences of the cognitive load on movement quality by the shape of a circle drawing when performed with and without an additional stroop task and found significant decline in movement quality in the dual task condition (Houwink et al., 2013).

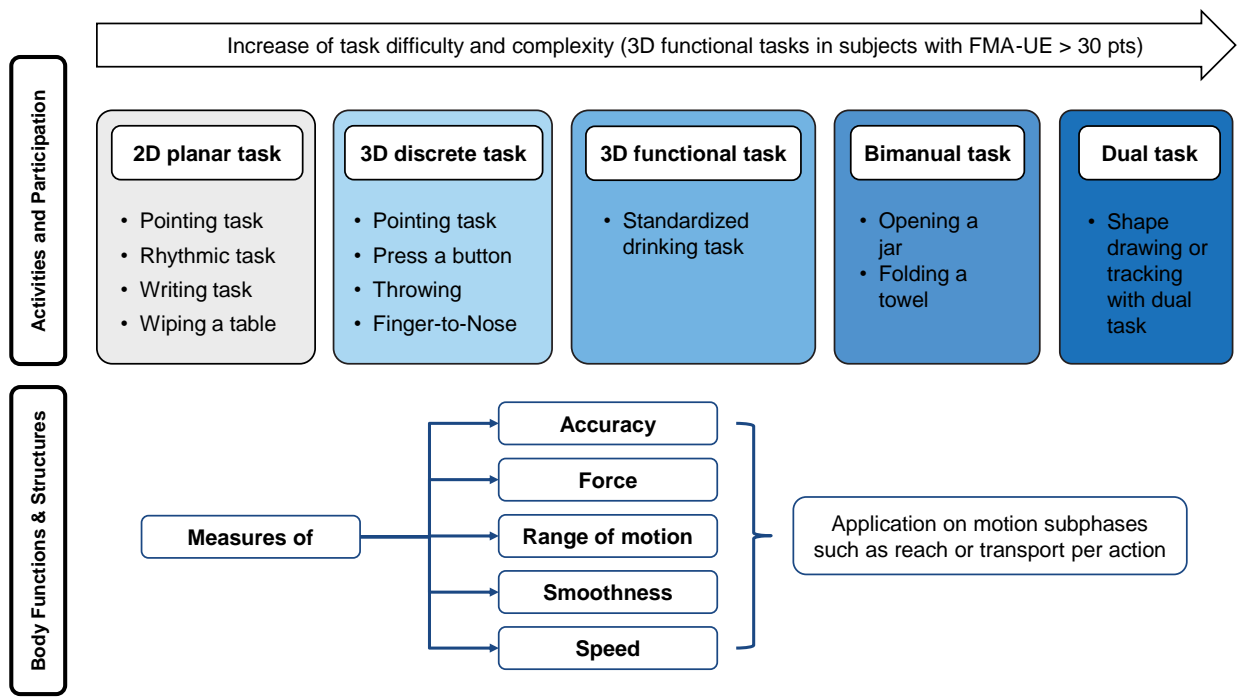

Figure 7.2. Considerations of upper limb movement task classifications for assessing upper limb functionality. 


\subsection{General conclusion}

This thesis addresses assessment opportunities of upper limb movement quality after stroke with prospects of wearable technologies, by considering the state of the art in upper limb kinematic assessments and performing experimental investigations on inertial sensing systems for quantification stroke-related of upper limb movements by use of kinematic metrics. Spatiotemporal measures, such as movement time, joint range of motions, peak velocity and smoothness measures were suggested for characterization of movement quality adding information to standard clinical scales. Kinematic assessments of functional movements that are close to real-world arm and hand usage were shown to be feasible, allowing to explore movement behaviour and quality on the level of sub phases, such as in motion primitives of reaching distally and proximally. Inertial sensing is a reasonable robust and easy-to-use method to apply qualitative movement analysis in different settings and task-constraints, hence helping to improve the understanding of pathomechanisms of stroke-related upper limb impairments on the body function level and the assessment opportunities across activities and participation in clinical practice of stroke rehabilitation.

\subsection{Future perspective}

The spinning out in technologies and wearable devices has been predicted to be the main future trend for rehabilitation applications besides human augmentation (Reinkensmeyer, 2019). Accordingly, future rehabilitation service would likely need to become more widespread in terms of cross-disciplinary teams including engineers besides health care professionals, patients, and their relatives to make effective use of technolgies, such as wearables. Sustainable collaboration between different clinical and technical experts is highly relevant for improvements in rehabilitative opportunities. The increased representativeness of engineering in rehabilitation is supported by an analysis of the National Institute of Health in 2018 that found bioengineer or rehabilitation engineer was most often listed in grant applications in rehabilitation between in the past eleven years (Reinkensmeyer, 2019). Likewise, education in clinical professions, such as physical or occupational therapists, would need to incorporate basic knowledge about technology-based opportunities for rehabilitation service.

On the level of the patient, the past years have indicated a growing interest of patients to use technologies in rehabilitation that is expected to grow. A survey amongst the over 50 years 
aged population in Switzerland showed that $20 \%$ of the participants used physical activity tracking (Seifert et al., 2017). These trends support the idea that persons like to be in control of their data, such as for monitoring their level of physical fitness. Ongoing discussions between the different interest groups will likely improve technology-based assessments and the selection of subject- and impairment-specific qualifiers to monitor the progress after stroke and the effectiveness of interventions. Based on the presented work, the following questions should be addressed to progress in technology-based upper limb assessments: Is there a common understanding between the therapist and the patient about what is the treatment target? What are the relevant outcomes to evaluate the target achievement? Would patients like to receive feedback based on such agreed outcomes?

The increased availability of wearable kinematic assessment technologies can improve future rehabilitation from several perspectives. The determination of precise measures on the level of body functions and structures during activities and performance in daily living will help to uncover what determines regain in motor function after stroke. Hypothesis that could be addressed in future, are whether wearable technologies enable ecologically valid assessment of upper limb movement quality in inpatient and outpatient settings alike and whether wearable technology eases monitoring and training efficiency in subjects dealing with stroke-related impairments.

Additionally, factors influencing the movement performance of stroke subjects, such as the posture or additional weight load, should be relevant in future research on interjoint coordination and probable approaches to break through the pathological joint coupling in extension to recent trials (Ellis et al., 2018). Poor sensorimotor control in the upper limb, such as due to stroke, is known to increase the risk for shoulder joint pathologies, decreased active range of motion and in exaggerated forms a shoulder hand syndrome (Dark et al., 2007). Open questions related to training impaired upper limb functionalities, as for example, if and when to train tasks in whole or parts, or which feedback to augment, can be addressed by use of kinematic assessment technology. The detection of movement pathologies in an objective manner can noteworthy improve clinical reasoning and decision-making in the treatment approach and thereby overcome the subjectivity in expert-based evaluations. Nevertheless, the interpretation and selection of the kinematic outcome parameters will require ongoing inclusion of knowledge from clinical experts and close interaction with the technology developers. 
The developments and applications of metrics on a granular level of specific motion aspects will be further improved alongside with new and robust algorithms for movement phase detection and activity-recognition. Taken together, wearable technology for upper limb assessments will help to generate person-specific measures for better personalization and precision of stroke rehabilitation (Patel et al., 2012). Such granular information likely leads to specific and relevant training and feedback, thereby supporting individuals with stroke-related upper limb impairments in relearning previously acquired skills with altered number and quality of resources (Muratori et al., 2013). Wearable technologies including sensitive parameters will likely expand in terms of home-based training solutions, such as the Armeo Senso Reward System (Wittmann et al., 2016; Widmer et al., 2018) or the Arm Usage Coach (Klaassen et al., 2015; Held et al., 2016), that enable the provision of measurement and movement-activity-based feedback by gamifications and vibratory stimuli in unsupervised situations. It can be assumed that patients benefit from greatly extended periods of training focusing on their specific needs (Krakauer et al., 2005). Improvements in accuracy of processing and robustness of measurements provided by wearables will be important for the patients' adherence to training and encouragement.

Finally, besides the outlined possibilities for future directions in wearable technology for assessing upper limb functions after stroke, other directions, such as prediction (Wang et al., 2014; De Lucerna et al., 2017; Repnik et al., 2018), human augmentation, assistive device development, and human-machine interfaces (Averta et al., 2020) will be of importance, especially in stroke subject with moderate to severe impairments without sufficient voluntary movement control.

\subsection{References}

Ada L, O’Dwyer N, O’Neill E. Relation between spasticity, weakness and contracture of the elbow flexors and upper limb activity after stroke: an observational study. Disabil Rehabil. 2006;28:8917. doi: 10.1080/09638280500535165

Alt Murphy M, Häger CK. Kinematic analysis of the upper extremity after stroke - how far have we reached and what have we grasped? Phys Ther Rev. 2015;20:137-55.

Alt Murphy M, Murphy S, Persson HC, Bergström UB, Sunnerhagen KS. Kinematic Analysis Using 3D Motion Capture of Drinking Task in People With and Without Upper-extremity Impairments. J Vis Exp. 2018;(133):57228. doi: 10.3791/57228

Averta G, Della Santina C, Valenza G, Bicchi A, Bianchi M. Exploiting upper-limb functional principal components for human-like motion generation of anthropomorphic robots. J Neuroeng Rehabil. 2020;17:63. doi: 10.1186/s12984-020-00680-8 
Bernstein N. The co-ordination and regulation of movements. Pergamon Press, Oxford; 1967.

Cheung VC, Turolla A, Agostini M, Silvoni S, Bennis C, Kasi P, Paganoni S, Bonato P, Bizzi E. Muscle synergy patterns as physiological markers of motor cortical damage. Proc Natl Acad Sci U S A. 2012;109:14652-6. doi: 10.1073/pnas.1212056109

Cuesta-Vargas AI, Galán-Mercant A, Williams JM. The use of inertial sensors system for human motion analysis. Phys Ther Rev. 2010;15:462-73. doi: 10.1179/1743288X11Y.0000000006

Dark A, Ginn KA, Halaki M. Shoulder muscle recruitment patterns during commonly used rotator cuff exercises: an electromyographic study. Phys Ther. 2007;87:1039-46. doi: 10.2522/ptj.20060068

De Lucena DS, Stoller O, Rowe JB, Chan V, Reinkensmeyer DJ. Wearable sensing for rehabilitation after stroke: Bimanual jerk asymmetry encodes unique information about the variability of upper extremity recovery. IEEE Int Conf Rehabil Robot. 2017;2017:160;2013-8.

Ellis MD, Carmona C, Drogos J, Dewald JPA. Progressive Abduction Loading Therapy with HorizontalPlane Viscous Resistance Targeting Weakness and Flexion Synergy to Treat Upper Limb Function in Chronic Hemiparetic Stroke: A Randomized Clinical Trial. Front Neurol. 2018;9:71. doi: 10.3389/fneur.2018.00071

Ellis MD, Lan Y, Yao J, Dewald JPA. Robotic quantification of upper extremity loss of independent joint control or flexion synergy in individuals with hemiparetic stroke: A review of paradigms addressing the effects of shoulder abduction loading. J Neuroeng Rehabil. 2016;13:95.

Held JP, Klaassen B, van Beijnum BF, Luft AR, Veltink PH. Usability Evaluation of a VibroTactile Feedback System in Stroke Subjects. Front Bioeng Biotechnol. 2017;4:98. doi: 10.3389/ fbioe.2016.00098

Hogan N, Sternad D. On rhythmic and discrete movements: reflections, definitions and implications for motor control. Exp Brain Res. 2007;181:13-30. doi: 10.1007/s00221-007-0899-y

Houwink A, Steenbergen B, Prange GB, Buurke JH, Geurts AC. Upper-limb motor control in patients after stroke: Attentional demands and the potential beneficial effects of arm support. Hum Mov Science. 2013;32:377-87.

Jeannerod M. The neural and behavioral organization of goal-directed movements. Oxford: Clarendon Press; 1990.

Kanzler CM, Rinderknecht MD, Schwarz A, Lamers I, Gagnon C, Held JPO, Feys P, Luft AR, Gassert $\mathrm{R}$, Lambercy O. A data-driven framework for selecting and validating digital health metrics: use-case in neurological sensorimotor impairments. NPJ Digit Med. 2020;3:80. doi: 10.1038/ s41746-020-0286-7

Klaassen B, Bartels PGH, van Beijnum BJF, Hermens H. The development and evaluation of an arm usage coach for Stroke survivors. 2015 9th International Conference on Sensing Technology (ICST), Auckland, 2015 (pp. 514-9). doi: 10.1109/ICSensT.2015.7438452

Klochkov AS, Khizhnikova AE, Nazarova MA, Chernikova LA. Pathological Upper Limb Synergies of Patients with Poststroke Hemiparesis. Neurosci Behav Physi. 2018;48:813-22. doi: 10.1007/ s11055-018-0634-0

Krakauer JW, Carmichael ST. Broken Movement. Neurobiology of Motor Recovery after Stroke. MIT Press; 2017.

Krakauer JW, Ghez C, Ghilardi MF. Adaptation to visuomotor transformations: consolidation, interference, and forgetting. J Neurosci. 2005;25:473-8. doi: 10.1523/JNEUROSCI.4218-04.2005 
Kwakkel G, van Wegen EEH, Burridge JH, et al. Standardized Measurement of Quality of Upper Limb Movement After Stroke: Consensus-Based Core Recommendations From the Second Stroke Recovery and Rehabilitation Roundtable. Neurorehabil Neural Repair. 2019;33:951-8. doi: $10.1177 / 1545968319886477$

Levin MF, Liebermann DG, Parmet Y, Berman S. Compensatory Versus Noncompensatory Shoulder Movements Used for Reaching in Stroke. Neurorehabil Neural Repair. 2016;30:635-46.

Li KY, Lin KC, Chen CK, Liing RJ, Wu CY, Chang WY. Concurrent and predictive validity of arm kinematics with and without a trunk restraint during a reaching task in individuals with stroke. Arch Phys Med Rehabil. 2015;96:1666-75.

Lu TW, Chang CF. Biomechanics of human movement and its clinical applications. Kaohsiung J Med Sci. 2012;28:S13-25. doi: 10.1016/j.kjms.2011.08.004

Maceira-Elvira P, Popa T, Schmid AC, Hummel FC. Wearable technology in stroke rehabilitation: towards improved diagnosis and treatment of upper-limb motor impairment. J Neuroeng Rehabil. 2019;16:142. doi: 10.1186/s12984-019-0612-y

McCrea PH, Eng JJ, Hodgson AJ. Biomechanics of reaching: clinical implications for individuals with acquired brain injury. Disabil Rehabil. 2002;24:534-41. doi: 10.1080/09638280110115393

McPherson JG, Chen A, Ellis MD, Yao J, Heckman CJ, Dewald JPA. Progressive recruitment of contralesional cortico-reticulospinal pathways drives motor impairment post stroke. J Physiol. 2018;596:1211-25. doi: 10.1113/JP274968

Mesquita IA, Fonseca PFPD, Borgonovo-Santos M, Ribeiro E, Pinheiro ARV, Correia MV, Silva C. Comparison of upper limb kinematics in two activities of daily living with different handling requirements. Hum Mov Sci. 2020;72:102632. doi: 10.1016/j.humov.2020.102632

Muratori LM, Lamberg EM, Quinn L, Duff SV. Applying principles of motor learning and control to upper extremity rehabilitation. J Hand Ther. 2013;26:94-102; quiz 103. doi: 10.1016/j. jht.2012.12.007

Patel S, Park H, Bonato P, Chan L, Rodgers M. A review of wearable sensors and systems with application in rehabilitation. J Neuroeng Rehabil. 2012;9:21. doi: 10.1186/1743-0003-9-21

Paulich M, Schepers M, Rudigkeit N, Bellusci G. Xsens MTw Awinda: Miniature WirelessInertialMagnetic Motion Tracker for HighlyAccurate 3D Kinematic Applications. https://www.xsens. com/hubfs/3446270/Downloads/Manuals/MTwAwinda_WhitePaper.pdf

Reinkensmeyer DJ. JNER at 15 years: analysis of the state of neuroengineering and rehabilitation. J Neuroeng Rehabil. 2019;16:144. doi: 10.1186/s12984-019-0610-0

Repnik E, Puh U, Goljar N, et al. Using Inertial Measurement Units and Electromyography to Quantify Movement during Action Research Arm Test Execution. Sensors. 2018;18:2767.

Robert-Lachaine X, Mecheri H, Larue C, Plamondon A. Validation of inertial measurement units with an optoelectronic system for whole-body motion analysis. Med Biol Eng Comput. 2017;55:60919. doi: $10.1007 / \mathrm{s} 11517-016-1537-2$

Rohrer B, Fasoli S, Krebs HI, Hughes R, Volpe B, Frontera WR, Stein J, Hogan N. Movement smoothness changes during stroke recovery. J Neurosci. 2002;22:8297-304. doi: 10.1523/ JNEUROSCI.22-18-08297.2002

Rose DK, Winstein CJ. Bimanual training after stroke: are two hands better than one? Top Stroke Rehabil. 2004;11:20-30. doi: 10.1310/NCB1-JWAA-09QE-7TXB. Erratum in: Top Stroke Rehabil. 2005 Spring;12(2):X. 
Santello M, Lang CE. Are movement disorders and sensorimotor injuries pathologic synergies? When normal multi-joint movement synergies become pathologic. Front Hum Neurosci. 20156;8:1050. doi: $10.3389 /$ fnhum.2014.01050

Scano A, Chiavenna A, Malosio M, Molinari Tosatti L, Molteni F. Muscle Synergies-Based Characterization and Clustering of Poststroke Patients in Reaching Movements. Front Bioeng Biotechnol. 2017;5:62. doi: 10.3389/fbioe.2017.00062

Schwarz A, Kanzler CM, Lambercy O, Luft AR, Veerbeek JM. Systematic Review on Kinematic Assessments of Upper Limb Movements After Stroke. Stroke. 2019;50:718-27. doi: 10.1161/ STROKEAHA.118.023531

Seifert A, Schomann A, Rietz C, Schelling. The use of mobile devices for physical activity tracking in older adults' everyday life. Digital Health. 2017;3: 2055207617740088.

Subramanian SK, Yamanaka J, Chilingaryan G, Levin MF. Validity of movement pattern kinematics as measures of arm motor impairment poststroke. Stroke. 2010;41:2303-8.

Tomita Y, Rodrigues MRM, Levin MF. Upper Limb Coordination in Individuals With Stroke: Poorly Defined and Poorly Quantified. Neurorehabil Neural Repair. 2017;31:885-97. doi: $10.1177 / 1545968317739998$

van Meulen FB, Reenalda J, Buurke JH, Veltink PH. Assessment of Daily-Life Reaching performance After Stroke. Ann Biomed Eng. 2015;43(2):478-86.

Villepinte C, Verma A, Dimeglio C, De Boissezon X, Gasq D. Responsiveness of kinematic and clinical measures of upper-limb motor function after stroke: A systematic review and meta-analysis. Ann Phys Rehabil Med. 2020:101366. doi: 10.1016/j.rehab.2020.02.005

Wang J, Yu L, Wang J, Guo L, Gu X, Fang Q. Automated Fugl-Meyer assessment using SVR model. 2014 IEEE Int Symp bioelectron bioinformatics. IEEE ISBB. 2014;2014:0-3.

Widmer M, Held JP, Wittmann F, Lambercy O, Lutz K, Luft AR. Does motivation matter in upper-limb rehabilitation after stroke? ArmeoSenso-Reward: study protocol for a randomized controlled trial. Trials. 2017;18:580. doi: 10.1186/s13063-017-2328-2

Wittmann, F, Held JP, Lambercy O, et al. Self-directed arm therapy at home after stroke with a sensorbased virtual reality training system. J NeuroEngineering Rehabil. 2016;13:75. doi: 10.1186/ s12984-016-0182-1

Wittmann F, Lambercy O, Gassert R. Magnetometer-Based Drift Correction During Rest inIMU Arm Motion Tracking. Sensors (Basel). 2019;19:1312. doi: 10.3390/s19061312

Wong WY, Wong MS. Trunk posture monitoring with inertial sensors. Eur Spine J. 2008;17:743-53.

Yao J, Chen A, Carmona C, Dewald JP. Cortical overlap of joint representations contributes to the loss of independent joint control following stroke. Neuroimage. 2009;45:490-9. 

Summary 

Stroke accounts for the largest cause for disability in daily life and is associated with upper limb sensorimotor impairments ranging from paralysis in severe cases over a certain level of loss of interjoint coordination and movement selectivity leading to compensatory movement behavior, as well as more subliminal deficits such as decreased movement time or fatigability. Measuring upper limb impairments after stroke is important to improve the understanding in the underlying pathomechanisms and identifying possible targets and treatment approaches. Assessments of upper limb function after stroke include a large number of different observer- and time-based clinical scales as well as an increasing number of different technologies for recording motion kinematics. Even though each assessment has its strength, such as the well-derived assessment construct in clinical scales like the Fugl-Meyer Assessment of the Upper Extremity (FMA-UE) or the continuous objective monitoring in technologies such as wrist worn accelerometers, the prescribed amount and heterogeneity of measures hampers the interpretability, comparability, and conclusion-drawing in this field of research. Additionally, assessments of the upper limb multi-joint coordination in the damaged nervous system remain challenging due to the complex and unknown mechanisms that interplay in the physiological and pathological system and the large amount of variability in upper limb usage. In this thesis, the strengths of both, the clinical scales, and the kinematic measurement technology, were combined to provide new, sensitive, and relevant strategies for assessing upper limb function quality and impairments after stroke.

The main goal of this thesis was to investigate upper limb movement quality after stroke by use of wearable kinematic sensing technologies with the intention to improve assessment opportunities for clinical practice and research. Five research questions were formulated to contribute to this issue and addressed in three individual studies (one systematic literature review, one cross-sectional observational study and one pilot study) that were the basis for chapters 2 to 6 . The questions were the following:

I. What is the state of the art in upper limb kinematic assessments in stroke survivors, including the assessment protocols and outcome parameters selected?

II. What are possible kinematic quantifiers of inter-joint coordination and how are they expressed in different task conditions?

III. Is there a difference in kinematic expressions, explained by the upper limb movement task, or the impairment level?

IV. Is it possible to identify comparable kinematic characterization of movement primitives or subphases across different activities of daily life?

V. What are the effects of arm load and target height on upper limb kinematics from the trunk to the finger digits during functional reach-to-grasp movements? 
In chapter 2, the state of knowledge in literature regarding upper limb kinematic assessments in stroke survivors is summarized. A Systematic Review on Upper Limb Kinematics after Stroke has been performed to identify the measurement systems, the assessment of the movement tasks and metrics frequently used in post-stroke upper limb kinematic assessments. Until September 2018, 225 studies were identified and allocated to 5 different tasks and 3 measurement system groups. Subsequently, investigations on the psychometric properties of the evaluated metrics within the included studies were summarized with respect to the measurement constructs and rated according to their summarized evidence. Thirty studies investigated clinimetrics of 62 metrics: reliability $(n=8)$, measurement error $(n=5)$, convergent validity $(n=22)$, and responsiveness $(n=2)$. The metrics task/movement time, number of movement onsets, number of movement ends, path length ratio, peak velocity, number of velocity peaks, trunk displacement, and shoulder flexion/extension received a sufficient evaluation for one clinimetric property. The chapter closes with recommendations on assessment tasks, reporting and metrics to include in post-stroke upper limb kinematic assessments, with the intention to improve standards and comparability in upper limb kinematic measurements after stroke.

The second to fourth research questions were addressed in the chapters 3 to 5 , based on the cross-sectional observational study on "assessing pathological synergies of upper limb function after stroke". Data of 26 chronic stroke subjects (>6-month post-stroke) with mild to moderate upper limb impairments, and five healthy age-matched subjects was included, who performed items of the FMA-UE and a set of 30 daily living tasks when being acquired with a full-body worn inertial sensing suit consisting of 17 inertial measurement units. The purpose of the study was to comprehensively capture and quantify physiological and pathological movement characteristics during upper limb non-functional and functional semi-structured activities of daily life in chronic stroke subjects by use of wearable, easyto-use, sensing technology. By recording different movements, like single-joint movements, gesture movements, and reach-to-grasp and manipulate objects representative for activities of daily life, it was aimed to capture the full content of upper limb functionality in terms of workspace, task complexity, and the degrees of freedoms actively involved. Chapter 3 focusses on the question, how the level upper limb interjoint coordination can be quantified when considering different task contents, the tested arm, and covariates, such as the dominant affected hand, upper limb function, and age. Spatiotemporal kinematic parameters of the shoulder-elbow-trunk complex were investigated in four discrete functional and non-functional movement tasks and related to clinical measures of upper limb interjoint 
coordination. It has been found that the kinematic parameters were largely influenced by the task, with non-functional movements of the FMA-UE representing different shoulderelbow relations when compared to functional movements with and without contact to the environment. The results highlight the need to assess ecologically valid movement tasks with varying levels of task complexity.

Chapter 4 focusses on a function-based analysis and related outcome measures of upper limb kinematic measures that were captured during a set of $30 \mathrm{arm}$ and hand activities of daily life to quantify movement complexity and the assumption of reduced movement variability and adaptability in subjects after stroke. Movement variability metrics based on functional PCA, such as the reconstruction error of PC's and the derived dissimilarity index, have found to support the hypothesis of diminished movement complexity in the affected arm when compared to the non-affected arm for all four subjects and in relation to the impairment severity per subject.

A kinematic core-set, based on the results presented in chapter 2 that reflects the main movement domains of spatiotemporal characteristics, efficiency, spatial, speed, and smoothness, was applied on the gesture and grasp movements of set of daily living activities in chapter 5. It was questioned in how far the kinematics were influenced by the factor of movement task and by the impairment level. The results confirmed task-related differences between gesture and grasp movements with larger shoulder joint motions and higher peak velocity in gestures besides larger trunk, forearm, and wrist motions and a higher number of velocity peaks in grasp movements. The impairment level had significant effects on trunk displacement, shoulder flexion, movement time and the number of velocity peaks. The other question tackled in chapter 5 was whether primitives of reaching distally and proximally showed consistent kinematic characteristics across movement tasks. During reach to grasp distally, comparable contributions of trunk and shoulder movements with consistently strong relations between shoulder, elbow joint motions and peak velocity were shown. In reach to gesture proximally or distally, comparable temporal expressions were found with consistent associations between movement time and the number of velocity peaks.

The question of the effects of arm load and target position on upper limb kinematics from the trunk to the finger digits during functional reach-to-grasp movements has been answered in chapter 6. A distributed inertial sensing system that captures movements from the trunk to the first three fingertips including fingertip force sensing, that was developed within the European project SoftPro, has been applied in a pilot usability study on ten chronic stroke 
subjects to assess functional upper limb movement performance during reach-to-grasp and displacement tasks. It was investigated whether displacement, target height and object weight have an effect on trunk compensation and shoulder, elbow, wrist, and finger flexion/ extension. Strong effects on all metrics were found for the tested arm and target height, while an increased object weight showed effects on trunk compensation. Correlations between metrics resulted in significant negative correlations between trunk compensation and elbow flexion-extension in the affected arm indicating pathological movement coupling. In the less-affected arm, correlations between wrist and finger flexion/extension were found that could be explained by the physiological synergy of wrist extension and finger flexion during grasping. The findings support the clinical usability of sensor-based motion analysis.

Addressing the five research questions, was aimed to support quality and standardization of kinematic measurements to assess movement quality of the upper limb. By answering the first question, an overview on assessment protocols, systems and measures has been provided in chapter 2. Based on this overview, recommendations for improving assessment standards were stated including suggestable metrics that showed available evidence of their validity, reliability, measurement error, and, or responsiveness. Kinematic outcomes reflecting upper limb interjoint coordination were investigated in functional and non-functional tasks revealing task-related differences in spatial and spatiotemporal parameters (chapter 3). In chapter 4, it has been proven in a subsample of four stroke subjects that interjoint coordination after stroke is associated with diminished movement complexity by use of mathematical dimensionality reduction methods. Considering qualitative motion analysis of daily life activities, spatiotemporal kinematics revealed task-specific differences in spatial, speed, and smoothness metrics (chapter 5). Additionally, consistent patterns of expressions and relations between spatiotemporal parameters were found during the movement sub phases of reach to grasp distally and proximal across different activities. Finally, kinematic and kinetic measures extended from the trunk to the first three digits have shown to be useful for qualitative motion analysis and for detecting effects of the object weight a target position on trunk compensation and flexion/extension ranges in the shoulder, elbow, wrist, and fingers during reach-to-grasp (chapter 6).

This thesis addresses assessment opportunities of upper limb movement quality after stroke with prospects of wearable technologies, by considering the state of the art in upper limb kinematic assessments and performing experimental investigations on inertial sensing systems for quantification stroke-related of upper limb kinematics. Spatiotemporal measures, such as joint range of motions, peak velocity and smoothness measures were suggested 
for characterization of movement quality adding information to standard clinical scales. Inertial sensing is a reasonable robust and easy-to-use method to apply qualitative movement analysis in different settings and task-constraints, hence improve the understanding of pathomechanisms of stroke-related upper limb impairments and the assessment opportunities in clinical practice of stroke rehabilitation. It could be demonstrated that a set-up of five inertial measurement units (IMUs), or eight IMUs with the fingers included, enables the qualitative analysis of spatial and temporal movement characteristics in the upper limb of stroke subjects.

Future work should be extended on automatic movement segmentation based on detection of motion primitives as the smallest scale building block of movements to allow overarching comparison of upper limb movements, as well as on improved fingertip force sensing in reach-to-grasp and manipulation movements to sensitively capture interactions with the environment. Once established, these measures could be further included by use of a minimized sensor-set in training solutions and allow sustainable and independent exercising. 



\section{Samenvatting}



Beroerte is de meest voorkomende oorzaak van beperkingen in het dagelijks leven en wordt in verband gebracht met sensorimotorische stoornissen van de bovenste extremiteiten, variërend van volledige verlamming in ernstige gevallen, een bepaald verlies van coördinatie van beweging over meerdere gewrichten of bewegingsselectiviteit en compenserend bewegingsgedrag tot minder expliciet duidbare gebreken, zoals een kortere trainingstijd of snellere vermoeidheid. Het meten van de stoornis van de bovenste ledematen na een beroerte is belangrijk om het begrip van de onderliggende pathofysiologische mechanismen te verbeteren en om mogelijke behandeldoelen en -methoden te identificeren. De instrumenten voor het meten van de functie van de bovenste extremiteit na een beroerte omvatten een groot aantal verschillende observatie- en tijdgebaseerde klinische schalen, evenals een toenemend aantal verschillende technologieën voor het registreren van bewegingen. Hoewel elk meetinstrument zijn sterke punten heeft, zoals het goed afgeleide evaluatieconstruct op klinische schalen zoals de Fugl-Meyer Assessment van de bovenste extremiteiten (FMA-UE) of de continue objectieve meting met sensoren, zoals om de pols gedragen versnellingsmeters, belemmeren het aantal en de heterogeniteit van de meetinstrumenten de interpreteerbaarheid, vergelijkbaarheid en conclusies op dit onderzoeksgebied. Bovendien blijft het beoordelen van de coördinatie van de bewegingen van de bovenste extremiteiten, bij betrokkenheid van meerdere gewrichten en bij een beschadigd zenuwstelsel een uitdaging vanwege de complexe en onbekende mechanismen die interacteren in het fysiologische systeem onder deze pathologische conditie en de grote variabiliteit in het gebruik van de bovenste extremiteit. In dit onderzoek werden de sterke punten van zowel klinische weegschalen als kinematische meetinstrumenten gecombineerd om nieuwe, gevoelige en relevante strategieën te bieden voor het beoordelen van de functionele kwaliteit en beschadiging van de bovenste extremiteit na een beroerte.

Het belangrijkste doel van dit onderzoek was om de kwaliteit van beweging van de bovenste extremiteit na een beroerte te bestuderen met behulp van draagbare kinematische sensortechnologieën ten einde de beoordelingsmogelijkheden voor klinische praktijk en onderzoek te verbeteren. Hiertoe zijn vijf onderzoeksvragen geformuleerd die in drie afzonderlijke studies (een systematische literatuurstudie, een cross-sectionele observationele studie en een pilotstudie) zijn behandeld. Dit vormt de basis voor de hoofdstukken 2 tot en met 6. Deze vragen luiden als volgt:

I. Wat is de stand van de vakliteratuur betreffende kinematische meetinstrumenten en beoordelingen van de bovenste extremiteit bij mensen na een CVA, inclusief de geselecteerde beoordelingsprotocollen, systemen en uitkomstparameters? 
II. Wat zijn de mogelijke kinematische uitkomstparameters voor het evalueren van de bewegingscoördinatie over meerdere gewrichten en in hoeverre worden deze verschillend uitgedrukt onder verschillende taakomstandigheden?

III. Is er een verschil in de resultaten van kinematische parameters die verklaard kunnen worden op basis van de uitgevoerde bewegingstaak en / of de mate van beperking in de bovenste extremiteit?

IV. Is het mogelijk om vergelijkbare kinematische kenmerken van bewegingsprimitieven of subfasen te identificeren over verschillende activiteiten van het dagelijks leven?

V. Welke effecten hebben de armbelasting en de doelhoogte op de kinematica van de bovenste extremiteit van romp tot vingertoppen bij functionele bewegingen met grijpfuncties?

Hoofdstuk 2 vat de kennis in de literatuur betreffende kinematische beoordelingen van de bovenste extremiteit bij overlevenden van een beroerte samen. Er is een systematische review van de literatuur over de kinematica van de bovenste extremiteit na een beroerte uitgevoerd om veelgebruikte meetsystemen, te beoordelen bewegingstaken en meetgegevens te identificeren. In september 2018 waren 225 onderzoeken geïdentificeerd, die waren gerelateerd aan vijf verschillende taakgroepen en drie meetsysteemgroepen. Als resultaat werd de informatie uit de onderzoeken naar de psychometrische eigenschappen van de beoordeelde metrieken in relatie tot de meetconstructen samengevat en beoordeeld op basis van het onderbouwende bewijs. Dertig studies onderzochten de klinimetrische eigenschappen van 62 metrieken: betrouwbaarheid $(n=8)$, meetfout $(n=5)$, convergente validiteit $(n=22)$ en reactievermogen $(\mathrm{n}=2)$. De metrieken "taak / bewegingstijd”, "aantal bewegingsaanzetten”, “aantal bewegingseinden”, “padlengteverhouding”, “pieksnelheid”, “aantal snelheidspieken”, "rompverplaatsing" en "schouderflexie" / extensie werden beoordeeld met voldoende kwaliteit voor ten minste één klinimetrische eigenschap. Het hoofdstuk wordt afgesloten met aanbevelingen over het gebruik van verschillende taken voor bewegingsbeoordeling, protocollen en meetgegevens die moeten worden opgenomen in de kinematische beoordelingen van de bovenste extremiteit na een beroerte met de bedoeling om de normen en vergelijkbaarheid van deze beoordelingen na een beroerte te verbeteren.

Onderzoeksvragen II t/m IV worden behandeld in de hoofdstukken 3 tot en met 5 op basis van een cross-sectionele observationele studie naar de "beoordeling van pathologische synergieën in de functie van de bovenste extremiteiten na een beroerte". Gegevens van 26 patiënten in het chronische stadium na een beroerte ( $>6$ maanden na een beroerte) met 
milde tot matige stoornissen van de bovenste extremiteit en vijf gezonde proefpersonen in dezelfde leeftijdscategorie werden geïncludeerd. De proefpersonen voerden individuele taken van de FMA-UE en een set van 30 dagelijkse taken uit, terwijl ze een sensorpak droegen met sensoren verspreid over het lichaam. Dit pak bevatte 17 traagheidssensoren. Het doel van de studie was om met behulp van draagbare, gebruiksvriendelijke sensortechnologie fysiologische en pathologische bewegingskenmerken tijdens niet-functionele en functionele, semi-gestructureerde activiteiten van het dagelijks leven bij patiënten met een chronische beroerte volledig vast te leggen en te kwantificeren. Door tijdens verschillende bewegingen te meten, zoals geïsoleerde gewrichtsbewegingen, gebarenbewegingen, evenals het grijpen en manipuleren van objecten die representatief zijn voor activiteiten van het dagelijks leven, werd de volledige functionaliteit van de bovenste extremiteit in relatie tot het werkgebied, de complexiteit van de taken en de actief betrokken vrijheidsgraden geregistreerd. Hoofdstuk 3 richt zich op de vraag hoe de bewegingscoördinatie over meerdere gewrichten van de bovenste extremiteit kan worden gekwantificeerd in relatie tot verschillen in taakinhoud, geteste arm en covarianten zoals dominantie van de aangedane hand, functionaliteit van de aangedane extremiteit en leeftijd. Spatiotemporele kinematische parameters van het schouder-elleboog-romp-complex werden onderzocht in vier discrete functionele en niet-functionele bewegingstaken en vergeleken met klinische meetinstrumenten voor de kwantificering van de bewegingscoördinatie over meerdere gewrichten van de bovenste extremiteit. Het bleek dat de kinematische parameters grotendeels werden beïnvloed door de taak. Daarbij lieten niet-functionele bewegingen van de FMA-UE andere relaties tussen schouder- en elleboogbewegingen zien dan functionele bewegingen met en zonder contact met de omgeving. De resultaten onderstrepen de noodzaak om ecologisch verantwoorde oefentaken met verschillende mate van complexiteit te beoordelen.

Hoofdstuk 4 concentreert zich op een functie-gebaseerde analyse en gerelateerde uitkomstparameters van kinematische metingen van de bovenste extremiteit, geregistreerd tijdens een reeks van 30 arm- en handactiviteiten van het dagelijks leven ten einde de bewegingscomplexiteit en de hypothese van verminderde bewegingsvariabiliteit en aanpassingsvermogen bij proefpersonen na een beroerte te bepalen. De uitkomstmetrieken van bewegingsvariabiliteit, gebaseerd op functionele Principale Componenten Analyse (PCA), zoals de reconstructiefout van principale componenten en een hiervan afgeleide index voor variabiliteit, hebben de hypothese van verminderde bewegingscomplexiteit in de aangedane arm in vergelijking met de niet-aangedane arm voor alle vier proefpersonen bevestigd, ook in relatie tot de ernst van de beperking van elke proefpersoon. 
Een kinematische kernset, gebaseerd op de resultaten gepresenteerd in hoofdstuk 2, die de belangrijkste bewegingsgebieden van spatiotemporele kenmerken, efficiëntie, ruimtelijke veranderingen, snelheid en soepelheid van bewegingen weerspiegelt, werd in hoofdstuk $\mathbf{5}$ toegepast op verschillende gebaren en grijpbewegingen van alledaagse arm- en handactiviteiten. Onderzocht werd in hoeverre de kinematica werd beïnvloed door de bewegingstaak en de ernst van de beperking. De resultaten bevestigden taakgerelateerde verschillen tussen gebaren en grijpbewegingen. Daarbij bleken bewegingen van het schoudergewrichtsbeweging groter en pieksnelheid hoger bij gebaren en romp-, onderarm- en polsbewegingen groter en het aantal snelheidspieken hoger bij grijpbewegingen. De ernst van de beperking had een significant effect op rompbewegingen, schouderflexie, bewegingstijd en het aantal snelheidspieken. De andere vraag die in hoofdstuk $\mathbf{5}$ aan de orde kwam, was of individuele bewegingsprimitieven bij reiken in distale en proximale richting vergelijkbare kinematische eigenschappen vertonen bij verschillende bewegingstaken. Bij het reiken in distale richting om te grijpen werden vergelijkbare bijdragen van romp- en schouderbewegingen getoond met sterke correlaties tussen bewegingen van schouder- en ellebooggewrichten en topsnelheid. Voor gebarenbewegingen in de distale of proximale richting werden vergelijkbare temporele resultaten gevonden met consistente associaties tussen de bewegingstijd en het aantal snelheidspieken.

De vraag naar de effecten van armbelasting en doelpositie op de kinematica van de bovenste extremiteit van de romp tot de vingertoppen tijdens functionele grijpbewegingen werd beantwoord in hoofdstuk 6. Een draagbaar systeem bestaande uit traagheidssensoren voor het registreren van bewegingen van de romp tot de eerste drie vingertoppen en vingertopdruksensoren voor het meten van de interactie tussen vingertoppen en omgeving, ontwikkeld als onderdeel van het Europese SoftPro-project, werd geëvalueerd in een oriënterende bruikbaarheidsstudie bij tien patiënten met een chronische beroerte. De functionele bewegingsprestaties van de bovenste extremiteiten werden geëvalueerd bij reiken om te grijpen en verplaatsingstaken. Onderzocht werd of de doelhoogte en het objectgewicht invloed hebben op zowel de rompcompensatie als de flexie en extensie van de schouder, elleboog, pols en vingers. Er werden sterke effecten op alle metrieken gevonden voor de factoren aangedane versus minder-aangedane arm en doelhoogte, terwijl een verhoogd objectgewicht effect leidde tot een verhoogde rompcompensatie. Verder werden significante negatieve correlaties gevonden tussen rompcompensatie en elleboogflexie-extensie in de aangedane arm, hetgeen wijst op pathologische bewegingssynergieën. In de minder aangedane arm werden correlaties gevonden tussen pols en vingerflexie-extensie, hetgeen verklaard kan 
worden door fysiologische bewegingssynergieën van polsextensie en vingerflexie bij het grijpen. De resultaten ondersteunen de klinische toepasbaarheid van bewegingsanalyse, gebruikmakend van sensoren.

Het doel van de beantwoording van de vijf onderzoeksvragen was om de kwaliteit en standaardisatie van bewegingsmetingen met traagheidssensoren te evalueren ten behoeve van de analyse van de bewegingskwaliteit van de bovenste extremiteiten. Bij beantwoording van de eerste vraag in hoofdstuk 2 is een overzicht gegeven van beoordelingsprotocollen, systemen en maatregelen. Op basis van dit overzicht zijn aanbevelingen gedaan voor verbetering van de beoordelingsnormen en voor metrieken met een voldoende validiteit, betrouwbaarheid en gevoeligheid, en een voldoende kleine meetfout. Kinematische metrieken die de bewegingscoördinatie over meerdere gewrichten van de bovenste extremiteit weerspiegelen werden in hoofdstuk 3 onderzocht voor functionele en niet-functionele taken. Hierbij werden taakspecifieke verschillen in spatiële en spatiotemporele parameters aangetoond. In hoofdstuk 4 werd in een subgroep van vier mensen die een beroerte hadden gehad met wiskundige dimenionaliteitsmaten aangetoond dat de complexiteit van de bewegingscoordinatie van gewrichten na een beroerte is verminderd. Kwalitatieve analyse van bewegingen tijdens het uitvoeren van activiteiten van het dagelijks leven, toonde taakspecifieke verschillen van spatiotemporele metrieken aan (hoofdstuk 5). Bovendien werden voor verschillende bewegingstaken vergelijkbare eigenschappen en relaties van spatiotemporele parameters gevonden tijdens reiken om te grijpen in distale en proximale richting. Ten slotte werd aangetoond dat kinematische en kinetische maten betreffende bewegingen variërend van de romp tot de eerste drie vingers nuttig zijn voor kwalitatieve bewegingsanalyse en de evaluatie van de effecten van objectgewicht en doelpositie op rompcompensatie en flexie-extensiebereiken van de schouder, elleboog, pols en vingers gedurende het reiken om te grijpen (hoofdstuk 6).

Dit proefschrift beschrijft de mogelijkheden om de bewegingskwaliteit van de bovenste extremiteit na een beroerte te evalueren door gebruikmaking van draagbare sensortechnologieën. Dit is gebaseerd op een inventarisatie van de huidige stand van kennis betreffende de kinematische analyse van de bovenste extremiteit en op experimenteel onderzoek met traagheidssensoren. Spatiotemporele uitkomstparameters zoals bewegingsbereik van een gewricht, pieksnelheid en maten voor de gladheid van bewegen werden voorgesteld voor de beoordeling van de bewegingskwaliteit, in aanvulling op standaard klinische schalen. Het meten van lichaamsbewegingen met inertiële bewegingssensoren is een verstandige, robuuste en gebruiksvriendelijke methode voor kwalitatieve bewegingsanalyses in verschillende 
omstandigheden en bij verschillende randvoorwaarden voor bewegingstaken en kunnen, daardoor, het begrip verbeteren van de pathomechanismen van beroerte-gerelateerde beperkingen van de bewegingssturing van de bovenste extremiteit en bijdragen aan de beoordeling van bewegingsbeperkingen na een beroerte in de klinische revalidatiepraktijk. Gebruik van vijf inertiële bewegingssensoren (IMU's) per arm en romp of acht IMU's als ook vingerbewegingen moeten worden geëvalueerd maken de analyse van de bewegingskwaliteit van de bovenste ledematen van patiënten na een beroerte mogelijk.

In de toekomst moet automatische bewegingssegmentatie verder worden onderzocht op basis van de detectie van bewegingsprimitieven, gedefinieerd als de kleinste eenheden van bewegingstaken, zodat bewegingen van de bovenste extremiteiten in een standaard stramien kunnen worden vergeleken. Ook is een verbeterde meting van de kinetische interactie tussen vingertoppen en omgeving nodig. Deze metingen kunnen vervolgens onderdeel uitmaken van een minimale sensorconfiguratie voor continue bewegingstherapie tijdens het dagelijks leven zonder voortdurende klinische begeleiding.. 



\section{Zusammenfassung}



Der Schlaganfall ist die häufigste Ursache für Einschränkungen des täglichen Lebens und geht mit sensomotorischen Störungen der oberen Extremitäten einher, die von kompletten Lähmungen in schweren Fällen, über einen bestimmten Verlust an Interjoint-Koordination oder Bewegungsselektivität und kompensatorischem Bewegungsverhalten bis hin zu unterschwelligeren Defiziten, wie zum Beispiel eine verringerte Bewegungszeit oder schnellere Ermüdbarkeit. Die Messung von Beeinträchtigungen der oberen Extremität nach einem Schlaganfall ist wichtig, um das Verständnis der zugrunde liegenden Pathomechanismen zu verbessern und mögliche Behandlungsziele und -ansätze zu identifizieren. Die Messinstrumente der Funktion der oberen Extremität nach Schlaganfall umfassen eine große Anzahl verschiedener beobachtungs-basierter und zeitbasierter klinischer Skalen, sowie eine zunehmende Anzahl verschiedener Technologien zur Aufzeichnung der Bewegungskinematik. Obwohl jedes Messinstrument seine Stärken hat, wie das gut abgeleitete Bewertungskonstrukt in klinischen Skalen wie dem Fugl-Meyer-Assessment der oberen Extremität (FMA-UE) oder die kontinuierliche objektive Überwachung bei Technologien, wie am Handgelenk getragenen Beschleunigungsmessern, die vorbeschriebene Menge und Heterogenität der Messinstrumente behindert die Interpretierbarkeit, Vergleichbarkeit und Schlussfolgerungen in diesem Forschungsbereich. Darüber hinaus bleibt die Beurteilung der Interjoint-Koordination der oberen Extremität im geschädigten Nervensystem aufgrund der komplexen und unbekannten Mechanismen, die im physiologischen und pathologischen System zusammenspielen, und der großen Variabilität der Verwendung der oberen Extremität, herausfordernd. In dieser Arbeit wurden die Stärken sowohl der klinischen Skalen als auch der kinematischen Messinstrumente kombiniert, um neue, sensitive und relevante Strategien zur Bewertung der Funktionsqualität und der Beeinträchtigungen der oberen Extremität nach einem Schlaganfall bereitzustellen.

Das Hauptziel der Arbeit war es, die Bewegungsqualität der oberen Extremität nach Schlaganfall mithilfe tragbarer kinematischer Sensortechnologien zu untersuchen, um die Assessmentmöglichkeiten für die klinische Praxis und Forschung zu verbessern. Fünf Forschungsfragen wurden formuliert, um zu diesem Thema beizutragen, und in drei Einzelstudien (eine systematische Literaturrecherche, eine Querschnitts-beobachtungsstudie und eine Pilotstudie) behandelt, die die Grundlage für die Kapitel 2 bis 6 bildeten. Die Fragen waren folgende:

I. Was ist der aktuelle Stand in der Fachliteratur zu kinematischen Messinstrumenten und Assessments der oberen Extremität bei Personen nach Schlaganfall, inklusive der ausgewählten Assessmentprotokolle, -systeme und Ergebnisparameter? 
II. Was sind mögliche kinematische Ergebnisparameter zur Bewertung der Interjoint-Koordination und inwiefern sind sie in verschiedenen Aufgabenbedingungen unterschiedlich ausgedrückt?

III. Gibt es einen Unterschied in den Ergebnissen kinematischen parameter, der durch die Bewegungsaufgabe und / oder den Grad der Beeinträchtigung in der oberen Extremität erklärt werden kann?

IV. Ist es möglich, vergleichbare kinematische Charakteristiken von Bewegungsprimitiven oder - unterphasen über verschiedene Aktivitäten des täglichen Lebens hinweg zu identifizieren?

V. Welche Auswirkungen haben die Armlast und die Zielhöhe auf die Kinematik der oberen Extremität vom Rumpf bis zu den Fingerbeeren während funktioneller Bewegungen mit Greiffunktionen?

In Kapitel 2 wird der Kenntnisstand in der Literatur zur kinematischen Assessments der oberen Extremität bei Schlaganfallüberlebenden zusammengefasst. Ein systematischer Review der Fachliteratur zur Kinematik der oberen Extremität nach einem Schlaganfall wurde durchgeführt, um häufig verwendete Messsysteme, die zu bewertenden Bewegungsaufgaben und Metriken zu identifizieren. Bis September 2018 wurden 225 Studien identifiziert, die fünf verschiedenen Aufgaben- und 3 Messsystemgruppen zugeordnet wurden. Daraus folgend wurden die Informationen der Untersuchungen zu den psychometrischen Eigenschaften der bewerteten Metriken in Bezug auf die Messkonstrukte zusammengefasst und anhand ihrer zusammengefassten Evidenz bewertet. 30 Studien untersuchten die klinimetrischen Eigenschaften von 62 Metriken: Reliabilität $(n=8)$, Messfehler $(n=5)$, konvergente Validität ( $n=22$ ) und Responsivität ( $n=2)$. Die Metriken "task/movement time”, "number of movement onsets", "number of movement ends", "path length ratio", "peak velocity”, "number of velocity peaks", "trunk displacement", und "shoulder flexion/extension" wurden mindestens für eine klinimetrische Eigenschaft mit ausreichender Qualität bewertet. Das Kapitel schließt mit Empfehlungen zum Einsatz von verschiedenen Assessment Bewegungsaufgaben, Protokollen und Metriken, die in die kinematischen Assessments der oberen Extremität nach einem Schlaganfall einbezogen werden sollen, mit der Absicht, die Standards und die Vergleichbarkeit der kinematischen Assessments der oberen Extremität nach einem Schlaganfall zu verbessern.

Die Forschungsfragen II bis IV wurden in den Kapiteln 3 bis 5 basierend auf der Querschnitts-beobachtungsstudie zur „Bewertung pathologischer Synergien der Funktion der oberen Extremitäten nach Schlaganfall“ behandelt. Es wurden Daten von 26 Patienten im chronischen Stadium nach Schlaganfall (>6 Monate nach Schlaganfall) mit leichten bis 
mittelschweren Beeinträchtigungen der oberen Extremität und fünf gesunden, altersentsprechenden Probanden eingeschlossen, die einzelne Aufgaben des FMA-UE und ein Set von 30 Aufgaben des täglichen Lebens ausführten, während sie einen Ganzkörper Sensoren-anzug, bestehend aus 17 Trägheitsmesseinheiten, tragen. Ziel der Studie war es, physiologische und pathologische Bewegungseigenschaften während nicht-funktioneller und funktioneller, semi-strukturierter Aktivitäten der täglichen Leben bei Patienten mit chronischem Schlaganfall mithilfe tragbarer, benutzer-freundlicher Sensortechnologie umfassend zu erfassen und zu quantifizieren. Durch die Aufzeichnung verschiedener Bewegungen, wie isolierter Gelenkbewegungen, Gestenbewegungen, sowie Greifen und Manipulieren von Objekten, die für Aktivitäten des täglichen Lebens repräsentativ sind, sollte der gesamte Inhalt der Funktionalität der oberen Extremität in Bezug auf den Arbeitsbereich, die Komplexität der Aufgaben, und die aktiv beteiligten Freiheitsgrade erfasst werden. Kapitel 3 konzentriert sich auf die Frage, wie die Interjoint-Koordination der oberen Extremität in Bezug auf unterschiedliche Aufgaben-inhalte, den getesteten Arm und Kovariaten, wie die dominante betroffene Hand, die Funktionaltät der betroffenen Extremität und das Alter, quantifiziert werden kann. Spatiotemporale kinematische Parameter des Schulter-EllbogenRumpf-Komplexes wurden in vier diskreten funktionellen und nicht-funktionellen Bewegungsaufgaben untersucht und mit klinischen Messinstrumenten zur Quantifizierung der Interjoint-Koordination der oberen Extremität verglichen. Es wurde festgestellt, dass die kinematischen Parameter weitgehend von der Aufgabe beeinflusst wurden, wobei nichtfunktionale Bewegungen der FMA-UE im Vergleich zu funktionellen Bewegungen mit und ohne Kontakt zur Umgebung unterschiedliche Schulter-Ellbogen-Korrelationen zeigen. Die Ergebnisse unterstreichen die Notwendigkeit, ökologisch-valide Bewegungsaufgaben mit unterschiedlicher Komplexität der Aufgaben zu beurteilen.

Kapitel 4 konzentriert sich auf eine funktionsbasierte Analyse und damit verbundene Ergebnisparameter kinematischer Messungen der oberen Extremität, die während des Sets von 30 Arm- und Handaktivitäten des täglichen Lebens erfasst wurden, um die Bewegungskomplexität und die Hypothese einer verringerten Bewegungsvariabilität und verminderten Anpassungsfähigkeit bei Probanden nach einem Schlaganfall zu quantifizieren. Die Ergebnisparameter der Bewegungsvariabilität, die auf funktioneller PCA basieren, wie der Rekonstruktionsfehler von PCs und der abgeleitete Unterscheidungsindex, haben die Hypothese einer verringerten Bewegungskomplexität im betroffenen Arm im Vergleich zum nicht betroffenen Arm für alle vier Probanden und in Bezug auf den Schweregrad der Beeinträchtigung pro Proband bestätigt. 
Ein kinematisches Core-set, basierend auf den in Kapitel 2 vorgestellten Ergebnissen, welches die Hauptbewegungsbereiche der spatiotemporalen Merkmale, Effizienz, räumliche Änderungen, Geschwindigkeit, und Geschmeidigkeit von Bewegungen widerspiegelt, wurde in Kapitel 5 auf verschiedene Gesten- und Greifbewegungen der alltäglicher Arm- und Handaktivitäten angewendet. Es wurde untersucht, inwieweit die Kinematik durch den Faktor der Bewegungsaufgabe und durch die Schwere der Beeinträchtigung beeinflusst wurde. Die Ergebnisse bestätigten aufgabenbedingte Unterschiede zwischen Gesten- und Griffbewegungen mit größeren Schultergelenkbewegungen und höherer Spitzengeschwindigkeit bei Gesten neben größeren Rumpf-, Unterarm- und Handgelenksbewegungen und einer höheren Anzahl von Geschwindigkeitsspitzen bei Greifbewegungen. Die Schwere der Beeinträchtigungen hatte signifikante Auswirkungen auf die Rumpfbewegungen, die Schulterflexion, die Bewegungszeit und die Anzahl der Geschwindigkeitsspitzen. Die andere in Kapitel 5 behandelte Frage war, ob einzelne Bewegungsprimitive (Grundeinheiten) in Form von Reichbewegungen in distaler und proximaler Richtung vergleichbare kinematische Eigenschaften über verschiedene Bewegungsaufgaben hinweg zeigen. Bei Reichbewegungen in distaler Richtung wurden vergleichbare Beiträge von Rumpf- und Schulterbewegungen mit aufgabenübergreifend starken Korrelationen zwischen Schulter-, Ellbogengelenkbewegungen und Spitzengeschwindigkeit gezeigt. Bei Gestenbewegungen in distaler oder proximaler Richtung wurden vergleichbare zeitliche Ergebnisse mit konsistenten Assoziationen zwischen der Bewegungszeit und der Anzahl der Geschwindigkeitsspitzen gefunden.

Die Frage nach den Auswirkungen der Armlast und der Zielposition auf die Kinematik der oberen Extremität vom Rumpf bis zu den Fingerbeeren während funktioneller Greifbewegungen wurde in Kapitel 6 beantwortet. Ein tragbareres System, bestehend aus Trägheitsmesseinheiten und Fingerbeeren-Drucksensoren, für das Erfassen das Bewegungen vom Rumpf bis zu den ersten drei Fingerspitzen, welches im Rahmen des europäischen Projekts SoftPro entwickelt wurde, fand in einer Pilot-Anwendbarkeits-Studie an zehn Patienten mit chronischem Schlaganfall Anwendung, um die funktionelle Bewegungsleistung der oberen Extremitäten bei Greifaufgaben zu bewerten. Es wurde untersucht, ob die Zielhöhe und das Objektgewicht einen Einfluss auf die Rumpfkompensation sowie die Flexion und Extension von Schulter, Ellbogen, Handgelenk und Finger haben. Starke Effekte auf alle Metriken wurden für die Faktoren, betroffener vs. weniger betroffener Arm und die Zielhöhe festgestellt, während ein erhöhtes Objektgewicht Effekte auf gesteigerte Rumpfkompensation zeigte. Korrelationen zwischen den Metriken resultierten in signifikanten negativen Korrelationen zwischen Rumpfkompensation und Ellenbogenflexion/-extension im betroffenen Arm, 
was auf pathologische Bewegungssynergien hinweist. Im weniger betroffenen Arm wurden Korrelationen zwischen Handgelenk- und Fingerflexion/-extension gefunden, die durch physiologische Bewegungssynergien von Handgelenkstreckung und Fingerflexion beim Greifen erklärt werden können. Die Ergebnisse unterstützen die klinische Anwendbarkeit von sensorgestützten Bewegungsanalysen.

Mit der Beantwortung der fünf Forschungsfragen sollte die Qualität und Standardisierung kinematischer Assessments zur Analyse der Bewegungsqualität in der oberen Extremität unterstützt werden. Durch Beantwortung der ersten Frage in Kapitel 2 wurde ein Überblick über Bewertungsprotokolle, -systeme und -maßnahmen gegeben. Basierend auf diesem Überblick wurden Empfehlungen zur Verbesserung der Assessmentstandards gegeben, einschließlich der Metriken, die aufgrund einer ausreichenden Evidenz bezüglich ihrer Validität, Reliabilität, Messfehler, und/ oder Responsivität empfohlen werden können. Kinematische Ergebnisparameter, die die Interjoint-Koordination der oberen Extremität widerspiegeln, wurden in funktionellen und nicht-funktionellen Aufgaben untersucht und aufgabenspezifische Unterschiede aufgedeckt (Kapitel 3). In Kapitel 4 wurde in einer Teilstichprobe von vier Schlaganfall-personen nachgewiesen, dass die Koordination von Gelenken nach einem Schlaganfall mit einer verringerten Bewegungskomplexität unter Verwendung mathematischer Dimensionsreduktions-methoden verbunden ist. Unter Berücksichtigung von qualitativen Bewegungsanalysen während des Ausführens von Aktivitäten des täglichen Lebens wurden aufgabenspezifische Unterschiede bezüglich der Ausprägungen von spatiotemporalen Ergebnissparametern beschrieben (Kapitel 5). Zusätzlich wurden vergleichbare Eigenschaften in den Ausprägungen und Verhältnissen spatiotemporaler Parametern während der Bewegungsgrundeinheiten, Reichen und Transportieren in distaler und proximaler Richtung, gefunden. Schließlich konnte gezeigt werden, dass kinematische und kinetische Messinstrumente, die vom Rumpf bis zu den ersten drei Fingern reichen, nützlich für die qualitative Bewegungsanalyse und die Untersuchung der Effekte des Objektgewichts und der Zielposition auf die Rumpfkompensation und die Schulter, Ellbogen, Handgelenk, und Finger Flexion/Extension sind (Kapitel 6).

Diese Arbeit beschreibt Möglichkeiten der Bewertung der Bewegungsqualität der oberen Extremität nach einem Schlaganfall in Hinblick auf tragbare Technologien unter Berücksichtigung des aktuellen Kenntnistandes zu kinematischen Analysen der oberen Extremität und auf der Basis experimenteller Untersuchungen mit Trägheitsmesseinheiten zur Quantifizierung der Bewegungsqualität der oberen Extremität nach einem Schlaganfall. Spatiotemporale Ergebnisparameter, wie das Gelenkbewegungs-ausmas, Spitzengeschwindigkeit 
und Smoothness oder Geschmeidigkeit wurden für das Assessment der Bewegungsqualität vorgeschlagen, um Informationen zu klinischen Standardskalen hinzuzufügen. Trägheitmesseinheiten stellen eine vernünftige, robuste und einfach zu verwendende Methode für die Erhebung qualitativer Bewegungsanalysen in verschiedenen Umgebungen und Aufgabenbeschränkungen dar und können dadurch das Verständnis der Pathomechanismen von Schlaganfall-bedingten Limitationen der oberen Extremität und die Assessmentmöglichkeiten in der klinischen Schlaganfallrehabilitations-praxis verbessern. Es konnte gezeigt werden, dass ein System aus fünf Trägheitsmesseinheiten (IMUs) oder acht IMUs inklusive der Finger, die Analyse der spatiotemporalen Bewegungsqualität in der oberen Extremität von Schlaganfallpatienten ermöglicht.

Zukünftige Arbeiten sollten auf die automatische Bewegungssegmentierung, basierend auf der Erkennung von Bewegungsprimitven oder -grundeinheiten, ausgedehnt werden, damit aufgaben-übergreifende Vergleiche von Bewegungselementen ermöglicht werden, sowie auch einer verbesserten Erfassung der Fingerspitzen-Druckkraft bei Greif- und Manipulationsbewegungen, damit sensibele Interaktionen mit der Umwelt erfasst werden können. Sofern erarbeitet, könnten diese Assessmentstrategien und Ergebnis-parameter weiter in technologie-basierte Trainingslösungen einbezogen werden und so ein nachhaltiges und unabhängiges Training ermöglichen. 



\title{
Acknowledgements Dankwoord
}

THANKS TO EVERYONE FOR YOUR SUPPORT

\author{
DANK AAN IEDEREEN \\ VOOR UW STEUN
}

DANKE AN ALLE FÜR IHRE UNTERSTÜTZUNG 



\section{About the author}





\section{Biography}

Anne Schwarz was born on May 10, 1986 in Neubrandenburg and grew up in Berlin, Germany. She attended the Käthe Kollwitz Gymnasium followed by the Carl-von-Ossjetzky Gymnasium in Berlin and graduated in 2005. From 2006 to 2008, she studied physiotherapy at the Reha Akademie Berlin and started working as a physiotherapist in the field of Neurorehabilitation in 2009. During the two years of work experience in statitionary rehabilitation at the Median Clinic Grünheide, Germany, several courses for

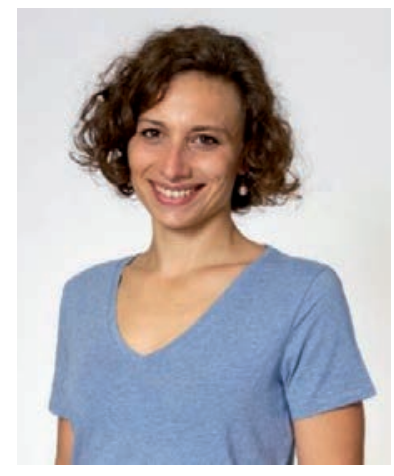
specialization in Neurorehabilitation were attended. In January 2011, Anne went abroad for studying physiotherapy towards a Master of Science at the School of Physiotherapy of the Otago University in Dunedin, New Zealand. Even though impressed by the physiotherapy education and service in New Zealand and the studies in musculoskeletal physiotherapy under the supervision of Dr. Steve Tumilty and Dr. Gill Johnson, she withdrew her course inscription after 6 weeks to continue her professional education in the field of neurology and rehabilitation. After returning to Germany, she gained further work experiences in different neurorehabilitation clinics, ambulant care settings and retirement homes, and started a Master of Science in Neurorehabilitation research at the Danube University Krems, Austria, in November 2011. In 2014, she graduated with her Masterthesis on "the effect of increased participation in goal setting in physiotherapy after stroke by use of goal attainment scaling". In 2016, Anne moved to Zurich, Switzerland to start working as a research physiotherapist and doctoral candidate in the group of Prof. dr. med. Andreas Luft at the division of Vascular Neurology and Neurorehabilitation, Department of Neurology, University of Zurich and University Hospital Zurich, Switzerland. This position was settled within European project SoftPro (Project No) spanning from 2016 to 2020 and associated with an external PhD candidate position at the Department of Biosignal and Systems, University of Twente, Enschede, Netherlands under supervision of Prof. dr. Jaap Buurke and Prof. dr. ir. Peter Veltink. During that period, she deepened her knowledge in biomechanics and upper limb motor control after stroke with a focus on aspects of interjoint coordination and technologybased measurement solutions, which coincided with several valuable collaborations with experts from fields such as bioengineering, physics and mathematics. Her drive to understand and influence physical functioning from the level of central nervous control to the level of biomechanical output and interplay kept influencing her professional career both practically and scientifically. 


\section{Publications}

\section{Peer-reviewed publications (thesis)}

Schwarz A*, Kanzler CM*, Lambercy O, Luft AR, Veerbeek JM. A Systematic Review on Kinematic Assessments of Upper Limb Movements after Stroke. Stroke. 2019;50:718-27. doi: 10.1161/STROKEAHA.118.023531

Schwarz A, Veerbeek JM, Held JPO, Buurke JH, Luft AR. Measures of interjoint coordination post-stroke across different upper limb movement tasks. Front Bioeng Biotechnol. 2021 Jan 28;8:620805. doi: 10.3389/fbioe.2020.620805

Schwarz A*, Averta G*, Veerbeek JM, Luft AR, Held JP, Valenza G, Bicchi A, Bianchi M. A functional analysis-based approach to quantify upper limb impairment level in chronic stroke patients: A pilot study. 2019 41st Annual International Conference of the IEEE Engineering in Medicine and Biology Society (EMBC). IEEE; 2019 (pp. 4198-204).

Schwarz A, Bhagubai MMC, Nies SG, Held JPO, Veltink PH, Buurke JH, Luft AR. Kinematic core-set of upper limb movements after stroke and their relationship across various upper limb activities of life. In preparation for submission to a peer-reviewed journal.

Schwarz A, Bhagubai MMC, Wolterink G, Held JPO, Luft AR, Veltink PH. Assessment of Upper Limb Movement Impairments after Stroke Using Wearable Inertial Sensing. Sensors. 2020;20:4770.

\section{Peer-reviewed publications (other)}

Bhagubai MMC*, Wolterink $G^{\star}$, Schwarz A, Held JPO, van Beijnum BJF, Veltink PH. Quantifying Pathological Synergies in the Upper Extremity of StrokeSubjects With the Use of Inertial Measurement Units: A pilot Study. Accepted for publication at IEEE Journal of Translational Engineering in Health \& Medicine.

Kanzler CM, Rinderknecht MD, Schwarz A, et al. A data-driven framework for selecting and validating digital health metrics: use-case in neurological sensorimotor impairments. NPJ Digit Med. 2020;3:80. doi: 10.1038/s41746-020-0286-7

Krakauer JW, Kitago T, Goldsmith J, Ahmad O, Roy P, Stein J, Bishop L, Casey K, Valladares B, Harran MD, Cortes JC, Forrence A, Xu J, Deluzio S, Held J, Schwarz A, et al. Comparing a novel neuroanimation experience to conventional therapy for high-dose, intensive upper-limb training in subacute stroke: The SMARTS2 randomized trial. medRxiv 2020.08.04.20152538; doi: 10.1101/2020.08.04.20152538. Under review. 
Kanzler CM, Schwarz A, Held JPO, et al. Technology-aided assessment of functionally relevant sensorimotor impairments in arm and hand of post-stroke individuals. J Neuroeng Rehabil. 2020;17:128. doi: 10.1186/s12984-020-00748-5

* the authors contributed equally

\section{Conference presentations}

Schwarz A, Held JP, Luft AR. Therapists' perspectives on new technologies for upper limb function after stroke - A focus group study. Conference Poster at NRNR 2017.

Schwarz A, Klaassen B, Luft AR, Veerbeek JM, Held JPO. Assessing upper limb interjoint coordination after stroke using inertial measurement units. Conference Talk at DGfB 2019.

Schwarz A, Kanzler CM, Luft AR, Lambercy O, Veerbeek JM. Systematic Review on upper limb kinematic assessments after stroke. Conference Poster and Talk in the Young Scientist Award Competition at NRNR 2019.

Schwarz A, Averta G, Veerbeek JM, Luft AR, Held JP, Valenza G, Bicchi A, and Bianchi M. A functional analysis-based approach to quantify upper limb impairment level in chronic stroke patients: A pilot study. Conference Talk at EMBC 2019.

Schwarz A, Freivogel S, Krüger C, Reuther J, Schönherr B, Knauss J. Effekte vermehrter Patientenbeteiligung an Zielsetzungen in der Physiotherapie nach Schlaganfall. Conference Talk at DGNR 2019 and nominated for the best short presentation.

Schwarz A, Bhagubai MMC, Wolterink G, Held JPO, Veltink PH. Wearable sensing system to detect movement quality and impairments during reach-to-grasp movements of subjects after stroke. Conference Poster at WCNR 2020. 



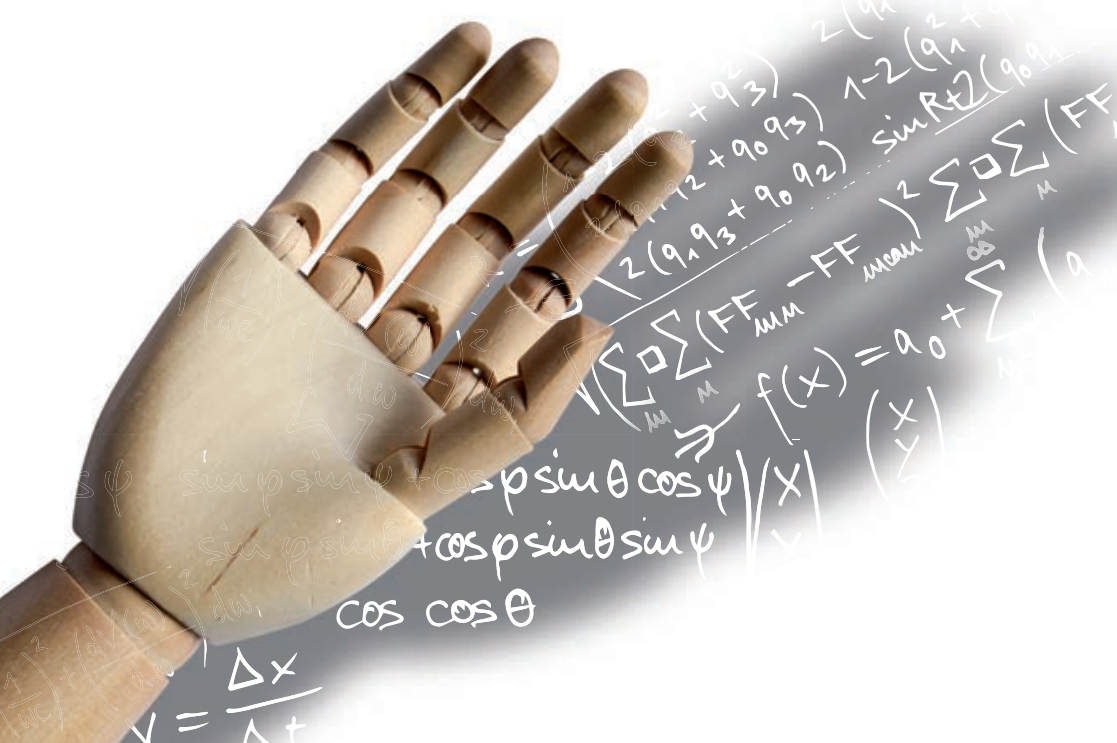

